\title{
Modelling and Stability Analysis \\ of a Composite Helicopter Rotor Blade with Integrated Active Fibres
}

\author{
by \\ Caroline R. Saxton, B.Sc. \\ Queen's University \\ A thesis submitted to \\ the Faculty of Graduate Studies and Research \\ in partial fulfillment of \\ the requirements for the degree of \\ Masters of Applied Science \\ Ottawa-Carleton Institute for \\ Mechanical and Aerospace Engineering \\ Department of \\ Mechanical and Aerospace Engineering \\ Carleton University \\ Ottawa, Ontario \\ April 11, 2003 \\ (C) Copyright \\ 2003 - Caroline R. Saxton
}


National Library

of Canada

Acquisitions and

Bibliographic Services

395 Wellington Street

Ottawa ON K1A ON4

Canada
Bibliothèque nationale

du Canada

Acquisisitons et services bibliographiques

395 , rue Wellington Ottawa ON K1A ON4 Canada
Your file Votre référence ISBN: 0-612-83532-4

Ourfile Notre référence ISBN: 0-612-83532-4
The author has granted a nonexclusive licence allowing the National Library of Canada to reproduce, loan, distribute or sell copies of this thesis in microform, paper or electronic formats.

The author retains ownership of the copyright in this thesis. Neither the thesis nor substantial extracts from it may be printed or otherwise reproduced without the author's permission.
L'auteur a accordé une licence non exclusive permettant à la Bibliothèque nationale du Canada de reproduire, prêter, distribuer ou vendre des copies de cette thèse sous la forme de microfiche/film, de reproduction sur papier ou sur format électronique.

L'auteur conserve la propriété du droit d'auteur qui protège cette thèse. $\mathrm{Ni}$ la thèse ni des extraits substantiels de celle-ci ne doivent être imprimés ou aturement reproduits sans son autorisation. 
The undersigned recommend to the Faculty of Graduate Studies and Research acceptance of the thesis

Modelling and Stability Analysis of a Composite Helicopter Rotor Blade with Integrated Active Fibres submitted by Caroline R. Saxton, B.Sc. in partial fulfillment of the requirements for the degree of Masters of Applied Science

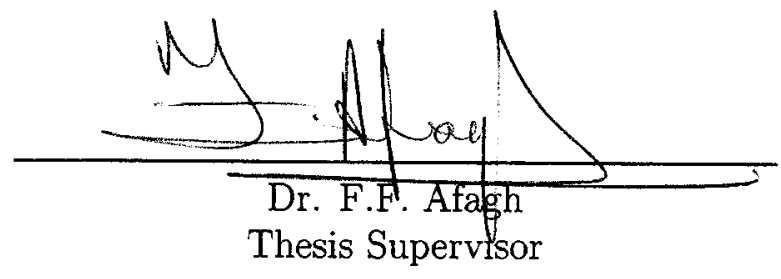

J. Beldoes -
Dr. J.C. Beddoes
Chair, Department of
Mechanical and Aerospace Engineering

Carleton University

May 1, 2003 


\begin{abstract}
This thesis is directed at a new structural configuration and design of helicopter rotor blades, termed a 'smart rotor'. The goal is to help reduce the large vibration levels in the rotorcraft during all types of helicopter maneuvers. The proposed design integrates standard fibre composite materials, that are currently in use in helicopter blades, with a new material type that can be described as an active fibre composite. This active composite is comprised of a layer of piezoelectric fibres in an epoxy matrix, sandwiched between two electrode sub layers. The active composite that is integrated into the host structure, when actuated, will cause an axial force as well as bending and twisting moments in the blade. These actuation 'forces' that are controllable, can be used to attenuate corresponding vibrations. The three main objectives of this thesis are to: develop the analytical expressions that describe the smart helicopter in hover, perform a study of an optimal blade design configuration, and analyse of the stability of the system.
\end{abstract}




\section{Acknowledgements}

I would like to express my thanks to my thesis advisor, Dr. Fred Afagh. Your guidance, knowledge, and positive attitude was much appreciated. To Dr. Fred Nitzsche, thanks for your insightful contributions. I would also like to express my thanks to the other professors and staff at the Department of Mechanical and Aerospace Engineering who also provided me with invaluable help.

Thanks to the other students at Carleton who also performed rotorcraft analyses S. Solaiman, B. Bailey, N. Morozova, and D. Opoku. Also, to my fellow graduate colleagues, thanks for the many amusing moments.

To Eva, Paul, Claudia, Stephen, Tina, and Matt, thanks for supporting me in all my decisions throughout university. 


\section{List of Symbols}

\begin{tabular}{|c|c|}
\hline$a$ & airfoil lift chord slope \\
\hline (a) & contribution due to actuation \\
\hline$a_{i}-e_{i}$ & cross-sectional constants \\
\hline$A$ & axial stiffness, blade structure cross-section area \\
\hline$A_{i}-O_{i j}$ & modal integrals \\
\hline$A_{i j}^{(a)}-J_{i j}^{(a)}$ & active modal integrals \\
\hline$A^{(a)}$ & axial stiffness contribution from actuator \\
\hline$A_{e I}, A_{e I I}$ & enclosed areas of left and right cells, respectively \\
\hline$A k_{A}$ & polar moment of inertia of blade cross-section \\
\hline$b$ & number of blades \\
\hline$B$ & coupling stiffness \\
\hline$B_{1}, B_{1}^{\star}, C_{1}^{\star}$ & cross-sectional constants \\
\hline$c$ & blade chord \\
\hline$c_{d}$ & airfoil profile drag coefficient \\
\hline$C$ & shear stiffness \\
\hline$C^{(a)}$ & shear stiffness contribution from actuator \\
\hline$C_{1}$ & warping rigidity \\
\hline$[C]$ & modal damping matrix \\
\hline$d$ & maximum cross-sectional dimension \\
\hline$d_{k i j}$ & piezoelectric electromechanical coupling tensor \\
\hline$D 3$ & applied voltage \\
\hline$D^{\alpha \beta \gamma \delta}$ & two-dimensional Hookean tensor \\
\hline$e$ & offset of mass axis from elastic axis \\
\hline$e_{A}$ & offset of tension axis from elastic axis \\
\hline
\end{tabular}




\begin{tabular}{|c|c|}
\hline$E$ & Young's modulus, ordering term \\
\hline$E_{e}, E_{k}$ & electric field \\
\hline$E^{i j k l}$ & three-dimensional Hookean tensor \\
\hline$f_{i}^{(a)}, g_{i}^{(a)}$ & asymptotical variable \\
\hline$F_{1}$ & axial force on blade \\
\hline$G$ & shear modulus \\
\hline$G^{\alpha \beta \mu}, G^{\gamma \delta \mu}$ & elastic constant \\
\hline$G(s)$ & asymptotical variable \\
\hline$h$ & cross-sectional thickness of cell wall \\
\hline$H_{\mu \lambda}$ & elastic constant \\
\hline$i, j, k$ & indices \\
\hline $\mathbf{i}_{x}, \mathbf{i}_{y}, \mathbf{i}_{z}$ & unit vectors in $x, y, z$ frame \\
\hline$I$ & potential energy, energy functional \\
\hline$I_{y^{\prime}}, I_{z^{\prime}}$ & cross-section area moments of inertia about the y' and z' axes, respectively \\
\hline$J$ & torsional constant including cross-sectional warping, polar moment of inertia \\
\hline$k_{A}$ & blade cross-section polar radius of gyration \\
\hline$k_{m}$ & blade cross-sectional mass radius of gyration \\
\hline$k_{m_{1}}, k_{m_{2}}$ & principal mass radii of gyration \\
\hline$K$ & dimensionless parameter \\
\hline$K_{i j}$ & stiffness constants \\
\hline$[K]$ & modal stiffness matrix \\
\hline$L$ & length of blade \\
\hline$L_{u}, L_{v}, L_{w}$ & distributed aerodynamic load expressions per unit length \\
\hline$m$ & mass per unit length \\
\hline$m_{1}, m_{2}, m_{3}$ & mass per unit length of spar and ballasts \\
\hline$M_{1}, M_{x^{\prime}}$ & torsion on blade \\
\hline
\end{tabular}




\begin{tabular}{|c|c|}
\hline$M_{2}, M_{y^{\prime}}$ & flap bending moment \\
\hline$M_{3}, M_{z^{\prime}}$ & lead-lag bending moment \\
\hline$M_{\phi}$ & generalized torsional aerodynamic moment per unit length \\
\hline$[M]$ & modal mass matrix \\
\hline $\mathbf{n}$ & normal vector \\
\hline$N$ & number of blades, number of modes \\
\hline$N R$ & non-rotating \\
\hline$N_{11}$ & axial stress resultant \\
\hline$N_{12}$ & shear flow resultant \\
\hline$N_{22}$ & hoop stress resultant \\
\hline$P_{i}$ & applied external load \\
\hline$P_{x^{\prime}}$ & work done by longitudinal stress \\
\hline$q_{i}$ & shear flow in ith branch \\
\hline $\mathbf{r}$ & position vector \\
\hline$r_{1}, r_{3}$ & radii of ballasts \\
\hline$R$ & radius of curvature of middle surface, blade radius \\
\hline$s_{1}, s_{2}, s_{3}$ & distances to centroid of spar and ballasts \\
\hline$s_{i j k l}^{E}$ & elastic compliance tensor at constant electric field \\
\hline$S_{x^{\prime}}$ & twisting moment due to shear \\
\hline $\mathbf{t}$ & tangential vector \\
\hline$t$ & time \\
\hline$T$ & kinetic energy, tension \\
\hline$T_{x^{\prime}}$ & twisting moment due to longitudinal stress \\
\hline$u_{i}$ & displacement field \\
\hline$u, v, w$ & displacements of the elastic axis in $\mathrm{x}, \mathrm{y}, \mathrm{z}$ directions \\
\hline$u_{x}, u_{y}, u_{z}$ & cartesian displacements \\
\hline
\end{tabular}




\begin{tabular}{|c|c|}
\hline$u(t)$ & excitation \\
\hline$\hat{U}, U$ & two-dimensional strain energy \\
\hline$U_{1}$ & average cross-sectional translation in $\mathrm{x}$-direction \\
\hline$U_{2}$ & average cross-sectional translation in $\mathrm{y}$-direction \\
\hline$U_{3}$ & average cross-sectional translation in z-direction \\
\hline$v_{1}^{(a)}(s)$ & asymptotical variable \\
\hline$v_{i}$ & induced inflow velocity \\
\hline$v_{\xi}, v_{s}, v_{l}$ & middle surface displacements \\
\hline$V$ & applied voltage \\
\hline$\vec{V}$ & velocity vector of an arbitrary point \\
\hline$V_{j}, W_{j}$ & lead-lag and flap bending generalized coordinates \\
\hline$V_{x^{\prime}}$ & axial stress resultant along $x^{\prime}$-axis \\
\hline$w_{i}$ & warping factors \\
\hline$W$ & work due to external loads \\
\hline$\{X\}$ & column vector of perturbation modal generalized coordinates \\
\hline$Y_{u}, Y_{v}, Y_{w}, Y_{\phi}$ & strain energy expressions \\
\hline$\xi, s, x$ & curvilinear coordinates \\
\hline$x, y, z$ & local Cartesian coordinates, undeformed \\
\hline$x^{\prime}, y^{\prime}, z^{\prime}$ & deformed coordinate system \\
\hline$X, Y, Z$ & global Cartesian coordinates \\
\hline$\hat{Z}_{u}, \hat{Z}_{v}, \hat{Z}_{w}, \hat{Z}_{\phi}$ & kinetic energy expressions \\
\hline$\alpha_{j}, \beta_{j}, \gamma_{j}$ & constants for assumed modes \\
\hline$\beta_{p c}$ & precone angle \\
\hline$\delta$ & Kronecker delta \\
\hline$\Delta$ & ordering term \\
\hline
\end{tabular}




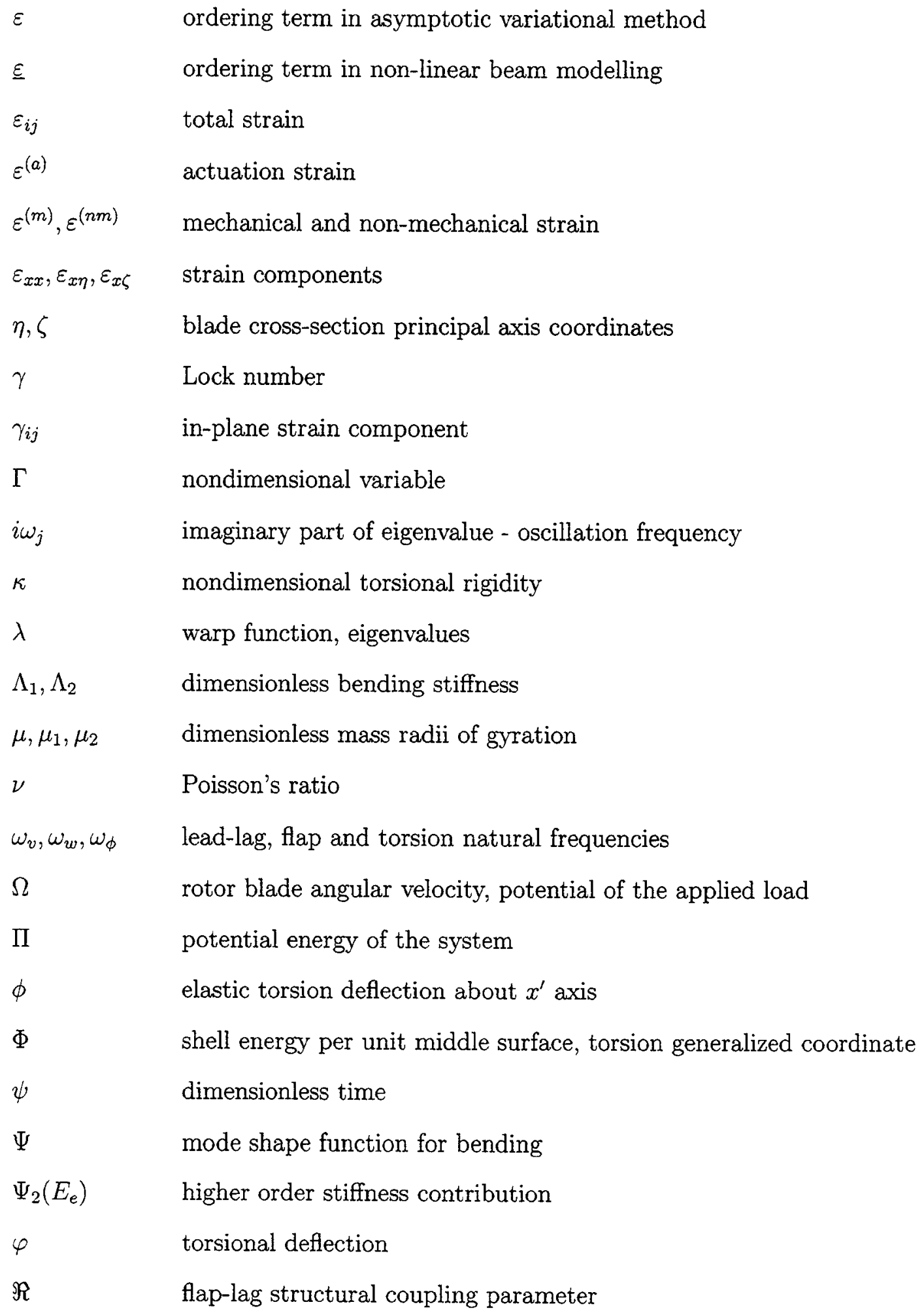




$\begin{array}{ll}\rho & \text { density of blade } \\ \rho_{\infty} & \text { density of air at sea level } \\ \rho_{i j} & \text { change in shell surface curvature } \\ \sigma & \text { blade solidity, real part of eigenvalue - growth/decay } \\ \sigma_{i j} & \text { stress tensor } \\ \sigma_{x x}, \sigma_{x \eta}, \sigma_{x \zeta} & \text { engineering stress components } \\ \theta & \text { blade pitch angle, ply angle } \\ \Theta_{j} & \text { torsional mode shape function } \\ & \\ ()^{\prime} & \partial / \partial x \\ \left(^{\cdot}\right) & \partial / \partial t \\ \left(^{-}\right) & \text {dimensionless quantity } \\ \langle(\bullet)> & \text { integral }\end{array}$




\section{Contents}

$\begin{array}{lll}\text { Acceptance } & \text { ii }\end{array}$

Abstract

Acknowledgements $\quad$ iv

List of Symbols $\quad$ v

Contents $\quad$ xi

List of Figures $\quad$ xv

List of Tables $\quad$ xix

1 Introduction 1

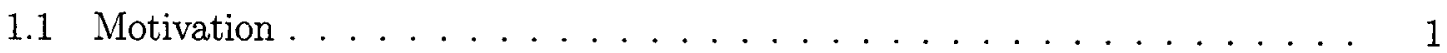

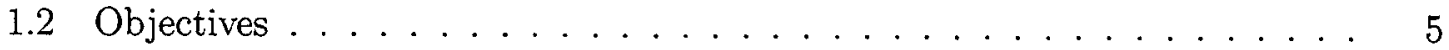

1.3 Background ........................... 6

1.3.1 Individual Blade Control . . . . . . . . . . . . . . . . 6

1.3.2 Active Fibre Composites . . . . . . . . . . . . . . . 12

1.4 Thesis Overview . . . . . . . . . . . . . . . . . . . . 14 
2 Cross-Sectional Analysis $\quad 16$

2.1 Reference Frame $\ldots \ldots \ldots \ldots \ldots$. . . . . . . . . . . . . . . . . . .

2.2 Shell Energy Functional . . . . . . . . . . . . . . . . . . . . . 19

2.2 .1 Ordering Scheme . . . . . . . . . . . . . . . 23

2.3 Variational Asymptotic Analysis . . . . . . . . . . . . . . . . . 24

2.4 Constitutive Relations . . . . . . . . . . . . . . . . . . . 29

3 1D Beam Analysis $\quad 31$

3.1 Hamilton's Principle . . . . . . . . . . . . . . . . . 34

3.1 .1 Reference Frame . . . . . . . . . . . . . . 34

3.1 .2 Strain Energy . . . . . . . . . . . . . . . 36

3.1 .3 Kinetic Energy . . . . . . . . . . . . . . . . 41

3.1.4 Virtual Work - Aerodynamic Loading . . . . . . . . . . . . . . 42

3.2 Differential Equations . . . . . . . . . . . . . . . . . 43

3.3 Nondimensional Equations . . . . . . . . . . . . . . . . . . 48

3.4 Galerkin's Method . . . . . . . . . . . . . . . . . 49

4 Blade Modelling $\quad 56$

4.1 Ply Orientation . . . . . . . . . . . . . . . . 58

4.1.1 Circumferentially Uniform Stiffness with Web, CUS/w . . . . . 60

4.1.2 Circumferentially Asymmetric Stiffness with Web, CAS/w . . . . 63

4.2 Modal Actuation . . . . . . . . . . . . . . . . . . 66

4.3 Validation of Code ...................... 67

4.4 Blade Modelling . . . . . . . . . . . . . . . . . . . . . 69

4.4.1 Design Specifications . . . . . . . . . . . . . . . 70

4.4 .2 Two-Celled Box Beam . . . . . . . . . . . . . . 72

4.4 .3 Two-Celled Blade . . . . . . . . . . . . . . . . . 74 
5 Stability and Response $\quad 83$

5.1 Case Studies . . . . . . . . . . . . . . . . . 86

5.2 Validation of Code . . . . . . . . . . . . . . 88

5.3 Steady-State or Equilibrium Deflections . . . . . . . . . . . . . . 92

5.3.1 Actuation over Blade Span . . . . . . . . . . . . . . . . 96

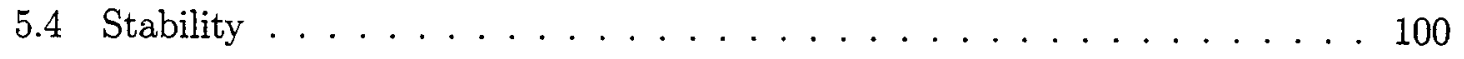

5.4 .1 Natural Frequencies . . . . . . . . . . . . . . . . . 100

5.4 .2 Response to Impulse . . . . . . . . . . . . . . . . . . 107

6 Summary, Conclusions and Recommendations 113

6.1 Summary . . . . . . . . . . . . . . . . . . . 113

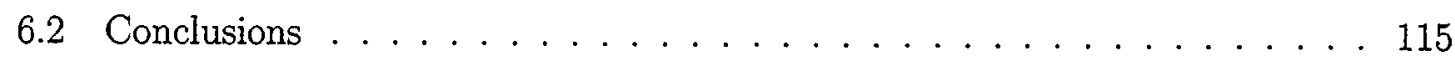

6.3 Recommendations . . . . . . . . . . . . . . . 116

$\begin{array}{ll}\text { References } & 118\end{array}$

$\begin{array}{ll}\text { Appendices } & 124\end{array}$

A Ordering Schemes $\quad 125$

A.1 2D Cross-Sectional Analysis . . . . . . . . . . . . . . 125

A.2 $1 \mathrm{D}$ Beam Formulation $\ldots \ldots \ldots \ldots \ldots \ldots$

B Cross-Sectional Constants $\quad 129$

C Linear Beam Formulation $\quad 134$

$\begin{array}{ll}\text { D Cross-Sectional Properties } & 147\end{array}$

E Actuation Vector Transformation $\quad 149$ 
F Nondimensional Constants and Galerkin Integrals

F.1 Nondimensional Constants . . . . . . . . . . . . . . . . . 152

F.2 Galerkin Integrals . . . . . . . . . . . . . . . . . 153

F.3 Segmented Actuated Galerkin Integrals . . . . . . . . . . . . . 154

F.4 Actuation from $x_{1}$ to $x_{2} \ldots \ldots \ldots \ldots \ldots$

G Perturbation Equations $\quad 156$

H Box Beam Variables $\quad 159$

I Trim Deflections $\quad 163$

$\begin{array}{lll}\mathrm{J} & \text { Lead-Lag and Flap Deflections } & 167\end{array}$

K Box Beam Code $\quad 174$

$\begin{array}{lll}\text { L NACA } 23012 \text { Airfoil Code } & 178\end{array}$

L.1 Blade Design Code . . . . . . . . . . . . . . . . . . . . 179

L.2 Stability Code . . . . . . . . . . . . . . . . . . . 182 


\section{List of Figures}

1.1 Blade load distribution . . . . . . . . . . . . . . . 2

1.2 Typical configuration of swashplate . . . . . . . . . . . . 4

1.3 Blade with trailing edge flap and piezostack actuators . . . . . . . . . 8

1.4 Side view of the smart spring blade . . . . . . . . . . . . 9

1.5 Pitch control by use of SMA torque tube . . . . . . . . . . . 9

1.6 Induced twist from piezoelectric actuation . . . . . . . . . . . . 10

1.7 Active fibre composite with interdigitated surface electrode pattern . . . . 12

2.1 Two-celled thin-walled blade cross-section . . . . . . . . . . . . . 18

2.2 Blade frames as derived by Hodges and Ormiston . . . . . . . . . . . 19

2.3 Branches for integration of a two-celled thin-walled cross-section . . . . . . 30

3.1 Possible hingeless rotor blade configurations . . . . . . . . . . 33

3.2 Elastic displacements . . . . . . . . . . . . . . . . . 34

3.3 Undeformed and deformed blade cross-sections between reference frames . 35

4.1 Circumferentially uniform stiffness configuration . . . . . . . . . 61

4.2 Lay-up sequences of top and bottom sides of CUS/w configuration . . . . 63

4.3 Circumferentially asymmetric stiffness configuration . . . . . . . . . . 64

4.4 Lay-up sequences of top and bottom sides of CAS configuration . . . . 65

4.5 Two-celled thin-wall box beam used for validation . . . . . . . . . . 68 
4.6 Two-celled box beam blade . . . . . . . . . . . . . . . 73

4.7 NACA 0012 blade section illustrating various box beam sizes . . . . . . 73

4.8 Two-celled blade design with a) actuator location and b) sectioning for parameter approximation . . . . . . . . . . . . . 76

4.9 Relationship between web position and stiffness in flap direction . . . . . 77

4.10 Relationship between web position and stiffness in lead-lag direction . . . . 78

4.11 Relationship between web position and torsional stiffness . . . . . . . . 78

4.12 Final concept no. $1 \ldots \ldots \ldots$. . . . . . . . . . . 81

4.13 Final concept no. $7 \ldots \ldots \ldots \ldots$. . . . . . . . . . . . 81

4.14 Final concept no. $8 \ldots \ldots \ldots \ldots \ldots$

5.1 Unsteady aerodynamic loads experienced by main rotor . . . . . . . . . . 84

5.2 Locus of roots for 1st lead-lag mode: $\omega_{v}=0.7, \omega_{w}=1.15, \beta_{p c}=0.0 \ldots 89$

5.3 Locus of roots for 1st flap mode: $\omega_{v}=0.7, \omega_{w}=1.15, \beta_{p c}=0.0 \ldots \ldots$

5.4 Locus of roots for torsion mode: $\omega_{v}=0.7, \omega_{w}=1.15, \beta_{p c}=0.0 \ldots \ldots$. . 90

5.5 Locus of roots for torsion mode: $\omega_{v}=0.7, \omega_{w}=1.15, \beta_{p c}=0.0 \ldots \ldots 1$

5.6 Locus of roots for a soft inplane rotor: Hodges and Ormiston . . . . . . . . 91

5.7 Equilibrium tip deflections in lead-lag bending . . . . . . . . . . . . 93

5.8 Equilibrium tip deflections in the flap bending . . . . . . . . . . . . . . 94

5.9 Equilibrium tip torsion . . . . . . . . . . . . . . . . . . 94

5.10 Torsional levels for the baseline case of Design $8 \ldots \ldots \ldots 7$

5.11 Torsional levels for zero applied load in Design $8 \ldots \ldots$. . . . . . . . 97

5.12 Torsional levels for $10 \%$ span-wise actuation in Design $8 \ldots \ldots$. . . . . . 98

5.13 Torsional levels for $25 \%$ span-wise actuation in Design $8 \ldots \ldots$. . . . . 98

5.14 Torsional levels for $50 \%$ span-wise actuation in Design $8 \ldots \ldots$. . . . 99

5.15 Torsional levels for $95 \%$ span-wise actuation in Design $8 \ldots \ldots 9$

5.16 Damping of the 1st lead-lag mode for Design 8a . . . . . . . . . . . 101 
5.17 Frequency of the 1 st lead-lag mode for Design $8 \mathrm{a} \ldots \ldots$. . . . . . . . 102

5.18 Damping for the 1st flap mode for Design 8a . . . . . . . . . . . 103

5.19 Frequency for the 1 st flap mode for Design 8 a . . . . . . . . . . 103

5.20 Damping for the 2nd flap mode for Design 8 a . . . . . . . . . . 105

5.21 Frequency for the 2nd flap mode for Design 8 a . . . . . . . . . . 105

5.22 Damping for the 1 st torsion mode for Design $8 \mathrm{a} \ldots \ldots$. . . . . . . 106

5.23 Frequency for the 1 st torsion mode for Design 8 a . . . . . . . . 106

5.24 Block diagram of the smart rotor blade . . . . . . . . . . . . 107

5.25 Sinusoidal input . . . . . . . . . . . . . . . . . . . . . . 109

5.26 Response to a unit-impulse function at $1800 \mathrm{~V},\{F\} \delta(t) \ldots \ldots . \ldots 111$

5.27 Response to a unit-impulse function at $900 \mathrm{~V},\{F\} \delta(t) \ldots \ldots . \ldots 11$

5.28 Response to a unit-impulse function at $1800 \mathrm{~V}$ for lead-lag . . . . . . . . 112

5.29 Response to a unit-impulse function at $900 \mathrm{~V}$ for lead-lag . . . . . . . . . 112

H.1 Box beam coordinates and shear flow parameters . . . . . . . . 160

I.1 Equilibrium tip deflections in lead-lag bending . . . . . . . . . . . . . . 164

I.2 Equilibrium tip deflections in the flap bending . . . . . . . . . . . . 164

I.3 Equilibrium tip torsion . . . . . . . . . . . . . . 165

I.4 Equilibrium tip deflections in lead-lag bending . . . . . . . . . . . . 165

I.5 Equilibrium tip deflections in the flap bending . . . . . . . . . . 166

I.6 Equilibrium tip torsion . . . . . . . . . . . . . . . 166

J.1 Lead-lag deflection for the baseline case of Design $8 \ldots \ldots \ldots 8$

J.2 Lead-lag deflection for zero applied load in Design $8 \ldots \ldots$. . . . . . 168

J.3 Lead-lag deflection for $10 \%$ span-wise actuation in Design $8 \ldots \ldots$. . . . 169

J.4 Lead-lag deflection for $25 \%$ span-wise actuation in Design 8 . . . . . . . 169

J.5 Lead-lag deflection for $50 \%$ span-wise actuation in Design $8 \ldots \ldots$. . . 170 
J.6 Lead-lag deflection for $95 \%$ span-wise actuation in Design $8 \ldots \ldots \ldots$

J.7 Flap deflection for the baseline case of Design $8 \ldots \ldots \ldots \ldots$

J.8 Flap deflection for zero applied load in Design $8 \ldots \ldots \ldots$. . . . . . 171

J.9 Flap deflection for $10 \%$ span-wise actuation in Design $8 \ldots \ldots$. . . . . 172

J.10 Flap deflection for $25 \%$ span-wise actuation in Design $8 \ldots \ldots$. . . . . 172

J.11 Flap deflection for $50 \%$ span-wise actuation in Design $8 \ldots \ldots 173$

J.12 Flap deflection for $95 \%$ span-wise actuation in Design $8 \ldots \ldots \ldots$

K.1 Program map for box beam blade . . . . . . . . . . . . . . . . 175

L.1 Program map for NACA 23012 blade . . . . . . . . . . . . . . . . . 179

L.2 Program map for stability analysis of NACA 23012 blade . . . . . . . . . 182 


\section{List of Tables}

2.1 Major ordering variables used in displacement functions . . . . . . . . . . 24

4.1 Stiffness constants of CUS/w configuration . . . . . . . . . . 62

4.2 Stiffness constants of CAS/w configuration . . . . . . . . . 65

4.3 Material properties of graphite-epoxy composite AS4/3501-6 . . . . . 68

4.4 Comparison of passive $K_{i j}$ stiffness constants for non-zero values . . . . . 69

4.5 Properties of scaled BO105 rotor blade . . . . . . . . . . . . . 70

4.6 Fixed properties for the BO105 blade . . . . . . . . . . . . . . . 72

4.7 Box beam designs . . . . . . . . . . . . . . . . . . 75

4.8 Ply orientations for passive blade designs . . . . . . . . . . . . . 77

4.9 Properties of Design $8 \ldots \ldots \ldots \ldots \ldots$

4.10 Characteristics of three final blade designs . . . . . . . . . . . 80

4.11 Result of final design concept for Shin . . . . . . . . . . . . . . . . 82

5.1 Plies on leading edge surface replaced by active plies in italic . . . . . . . 86

5.2 Material properties of AFC with the piezoelectric PZT-5H . . . . . 87

5.3 Stiffness values for Design $8 \ldots \ldots \ldots \ldots \ldots$

5.4 Parameters used for validation study . . . . . . . . . . . . . . 89

5.5 Torsion experienced at tip by smart blade designs . . . . . . . . . . . 95

I.1 Stiffness values for Designs 1 and $7 \ldots \ldots \ldots 3$ 


\section{Chapter 1}

\section{Introduction}

High vibration levels that are typical of helicopters today present an ergonomic and performance challenge. However, by providing a strong level of control authority in order to attenuate these vibrations, the resulting enhancements in these already agile aircraft are well merited. This thesis explores one such solution by virtue of a 'smart blade' that addresses the problem at the root. Computer-aided and numerical modelling tools are employed to substantiate the required level of authority for a hover flight condition. Moreover the new smart blade design, as a requisite, exhibits as good or better stability characteristics as the current 'passive' blades.

\subsection{Motivation}

A solution to counteracting the negative effects that vibrations cause on helicopter frames has been vigorously addressed throughout the past two decades beginning with the work of Loewy [1] and Friedmann [2]. The source of the vibrations lies in the complex and unsteady aerodynamic airloads that the main rotor is subject to throughout its rotation. The airloads are periodic in nature and are transmitted from the rotor blades down through to the helicopter's fuselage. For an N-bladed rotor with rotational speed, $\Omega$, these loads 


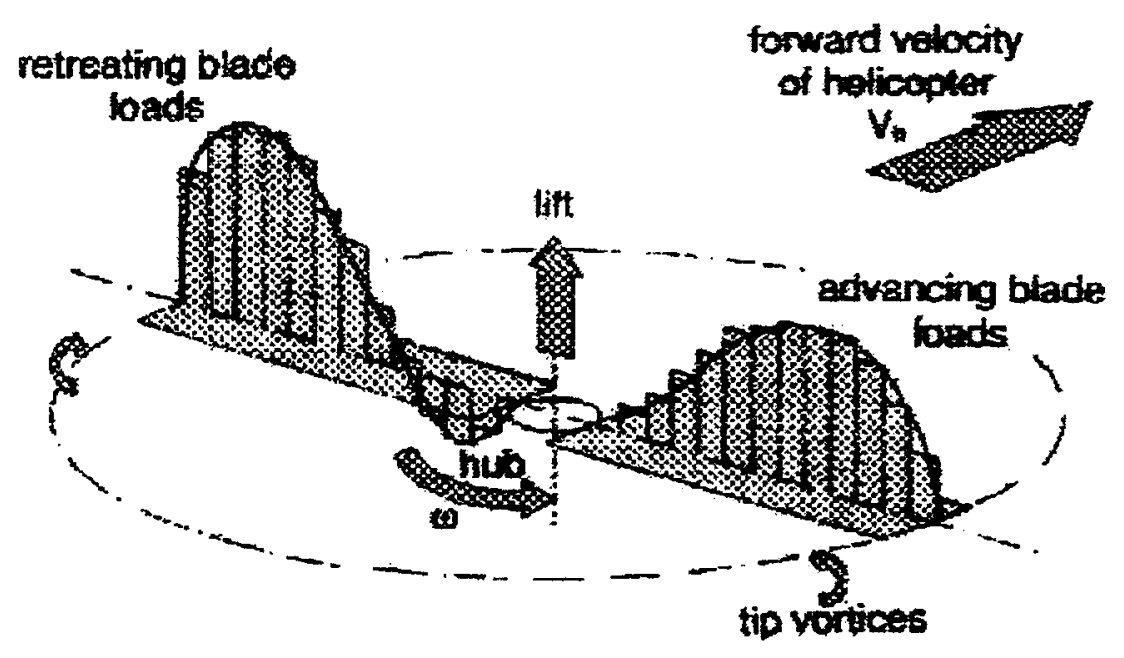

Figure 1.1: Blade load distribution

are transmitted in the form of $(\mathrm{N}+1) \Omega, N \Omega$, and $(\mathrm{N}-1) \Omega$ loads that result in $N \Omega$ forces on the fuselage [1]. Figure 1.1 illustrates the typical aerodynamic force distribution across a blade span for advancing and retreating points of rotation [3]. For example, a high Mach number on the advancing side of the blade tip contributes to aerodynamic instabilities, and on the retreating side the blade experiences blade stall and reverse flow effects. Many of the forces and moments cancel each other out when added together at the rotor hub, however the other vibrational loads are a concern. Increased vibration damping brings about a number of benefits including: performance enhancement in not only cruise speed but also payload, decreased fatigue levels of components and therefore less maintenance, decreases in operating costs, improvements in the quality of the ride, and lower acoustic noise.

The disturbances that cause the vibrations are most severe during transitional and forward flight. In studying hover flight however, a basis is established to facilitate analysis for these other flight conditions. Passive vibration control methods were first investigated by researchers, which are still commonly used today. Two types of passive vibration control methods were explored, isolators and absorbers (or diffusers) $[1,4]$. 


\title{
Isolators
}

Isolators separate the parent system from the source of vibration, i.e. by isolating the fuselage from the rotor blades. This is typically accomplished by suspending the fuselage with springs from the rotor system. The effect is that the resulting natural frequency will be low compared to the excitation frequency. One common isolator device is DAVI (Dynamic Antiresonant Vibration Isolator).

\begin{abstract}
Absorbers
Dynamic vibration absorbers are the most common of this type of passive vibration attenuation system. In this system, the natural frequency of a relatively small mass on a spring is tuned to the excitation frequency. This enables the small mass to vibrate in resonance with the excitations, thereby cancelling them out.
\end{abstract}

There are problems with using passive vibration devices. The most prominent of these problems is the reduction in performance away from the tuned aerodynamic flight condition, which is usually the forward flight speed. They also are limited when any of the dynamic parameters of the helicopter system are changed, e.g. due to fuel, cargo, or passenger weight changes. Also, the use of isolators or absorbers can add as much as $3 \%$ of the gross helicopter weight. Absorbers and isolators used within the fuselage itself (for seats, equipment, cargo/passenger floors) need to be low friction devices. These particular applications have the possibility of 'bottoming out' in some extreme manoeuvres if the spring stiffness is too low. These various factors contribute to an increase in cost and a decrease in relative effectiveness. 


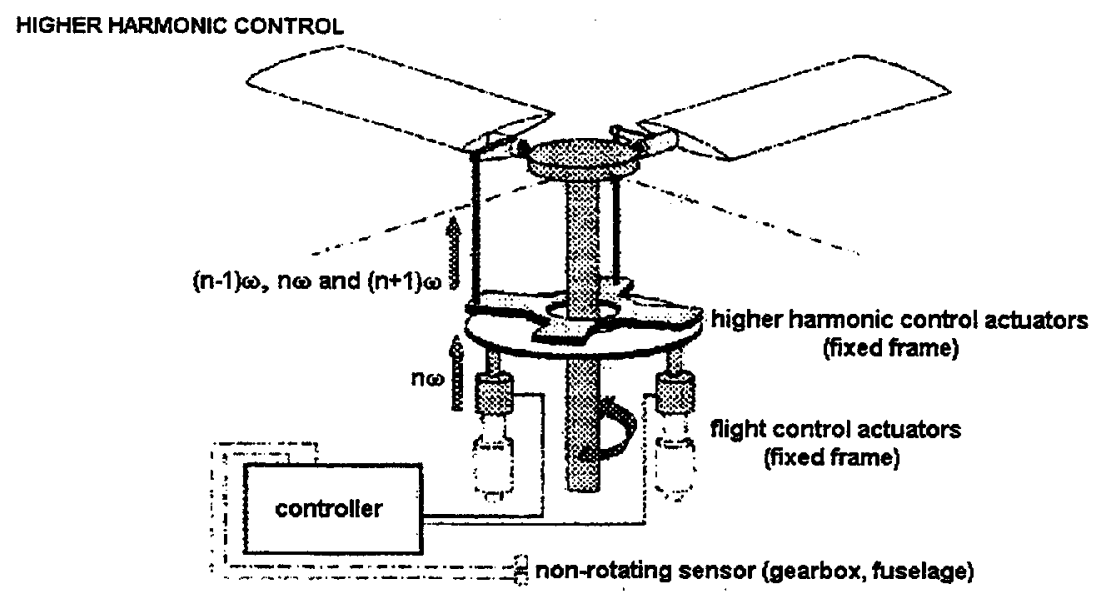

Figure 1.2: Typical configuration of swashplate

\section{Higher Harmonic Control}

An alternative approach was undertaken by Shaw and Albion [5], which was termed higher harmonic control, or $\mathrm{HHC}$, in order to address the problem in a more active way. HHC uses high frequency swashplate actuation for pitch control. The swashplate, which is the bulky device at the hub used for cyclic control as shown in Figure 1.2 [3], is primarily used for conventional flight control. However by exciting the swashplate at a frequency of $\mathrm{N} / \mathrm{rev}$, the transmitted harmonic $\mathrm{N} \Omega$ blade forces can be suppressed. Unfortunately this vibration damping device also has limitations. This mechanism is limited to the $\mathrm{N} / \mathrm{rev}$ pitch frequencies. Since HHC generates new loads on the blade that counteract the blade loads, at extreme flight conditions these loads become very large. Therefore the power needed by the servoactuators to drive the quick motion of the swashplate is also extreme. The effectiveness of HHC in these situations is downplayed by the lower suppression levels achieved, high power requirements, and resulting fatigue. In eliminating the swashplate there will be benefits in area reduction, drag, and weight, where typical weight penalties are approximately $1-2 \%$ of the gross weight.

A decade ago, initial steps were undertaken to consider active attenuation of rotor 
blade vibration by adopting smart structures technology rather than passive methods and HHC. The broad category to which the smart structures have been applied is classified as individual blade control, or IBC. In this approach, rather than frequency control at the swashplate, the blade itself is modified for pitch actuation. This allows for greater actuation authority at arbitrary frequencies and higher bandwidths. Since improvements in design are also achieved through the use of composites, their application in conjunction with smart structures improves the ability to vary the span-wise pitch angle.

\subsection{Objectives}

The first objective of this thesis is to model the dynamics of a two-celled, thin-walled, composite and hingeless smart blade. As a vibration attenuation scheme that falls under individual blade control, the blade has embedded piezoelectric fibres along its span. Two formulations are needed to describe the blade dynamics. The first is a variational asymptotic method that models the structural characteristics of the blade cross-section. This analysis sets up the forces and moments arising from the piezoelectric effect. The second is a nonlinear beam formulation along the elastic axis to take into account bending, warping, and torsion. Hamilton's equation is used to obtain the equations of motion by considering the contributions due to elastic strain and kinetic energies, as well as the aerodynamic loads. As an initial attempt, only a hover flight condition is considered.

The second objective of this thesis is to design and develop an approximate analytical blade model to be used in investigating the structural and dynamic characteristics of a typical smart blade. The models for the smart blade are based on a Mach-scaled version of a typical passive helicopter blade. Basing the proposed models on a passive blade ensures that the structural and aerodynamic properties have been retained in the proposed smart blade. The blades are to be composed of composite plies with various layers of integrated 
piezoelectric plies. Part of the modelling process involves an analysis of the composite layup, the ply orientations, and the number of inactive and active plies. Essentially, investigating the effect of the structural properties of the blade to produce an optimal design configuration, shows how the tailoring of a composite blade achieves enhancements in design.

The third and final objective of this thesis is to carry out a stability and preliminary response analysis of the smart blade designs. A smart blade should exhibit little or no degradation in performance or stability compared to a typical passive blade. The natural frequencies of the blade indicate whether the blade has been designed to be inherently stable. These frequencies depend on dynamic characteristics of the blade that are functions of web location, pitch angle, and percentage of the blade actuated, amongst other parameters. Recalling that aerodynamic loads on the blade are periodic in nature, the applied voltage sent to the embedded piezoelectrics will also be a function of azimuth location. This signifies that the use of an AC current will best dampen the vibrations directly. Therefore under this condition, the blade should also exhibit stability. As a prelude to future work on the design of an appropriate control law, the actuation authority of the smart blade is also investigated.

\subsection{Background}

\subsubsection{Individual Blade Control}

The idea behind both HHC and IBC is to control the aerodynamic forces that act on the helicopter rotor blade by varying the blade pitch. Shaw and Albion, in their work, showed that approximately $\pm 3^{\circ}$ of pitch change is needed with $H H C$ in order to suppress vibrations. Using this as a basis, IBC was shown to have greater potential for controlling span-wise lift; each individual blade provides pitch actuation. Furthermore, IBC is not limited to 
actuation at $\mathrm{N} / \mathrm{rev}$ frequencies that swashplates are restrained to. Periodic actuation can address the various vibrations experienced by the blade throughout its rotation.

There are several advantages that IBC has over $\mathrm{HHC}$, the main attraction lying in the decrease in weight and complexity from the removal of the swashplate mechanism. This approach is also a more direct one compared to the passive blade techniques - vibrations are attacked at the root source, not through a dozen other mechanical linkages to somewhere in the fuselage. The methods that will be discussed briefly below also are mechanically simple and only require electrical power.

There are two approaches in IBC that change blade pitch angle. They are: changing the aerodynamic shape of the lifting surface, and changing the angle of attack of the lifting surface. As an analogy, in wing surfaces of airplanes, the flaps and ailerons are the structures that change the airflow around these surfaces in order to change the aerodynamic forces acting upon them. Several schemes are currently being investigated for helicopter blade pitch control, each with their own advantages and disadvantages.

\section{Trailing Edge Flap}

Actuation that produces deflection of a trailing edge flap on a rotor blade is achieved by taking advantage of the large forces produced from piezostacks. These piezostack devices have high actuation forces but low actuation strokes signifying that an amplification mechanism is needed to transfer the stroke of the piezostack in order to produce flap deflection. Figure 1.3 illustrates one example of the mechanism $[6,7]$. This is one of the more commonly used methods being investigated today for rotor blade vibration suppression. It is a mechanically simple and modular method that requires little power consumption, however degradation in performance in the stroke occurs due to high centrifugal forces in the rotating environment. One other major drawback in this approach is the large increase in profile drag during deflection. For further detailed information on the concept of trailing 


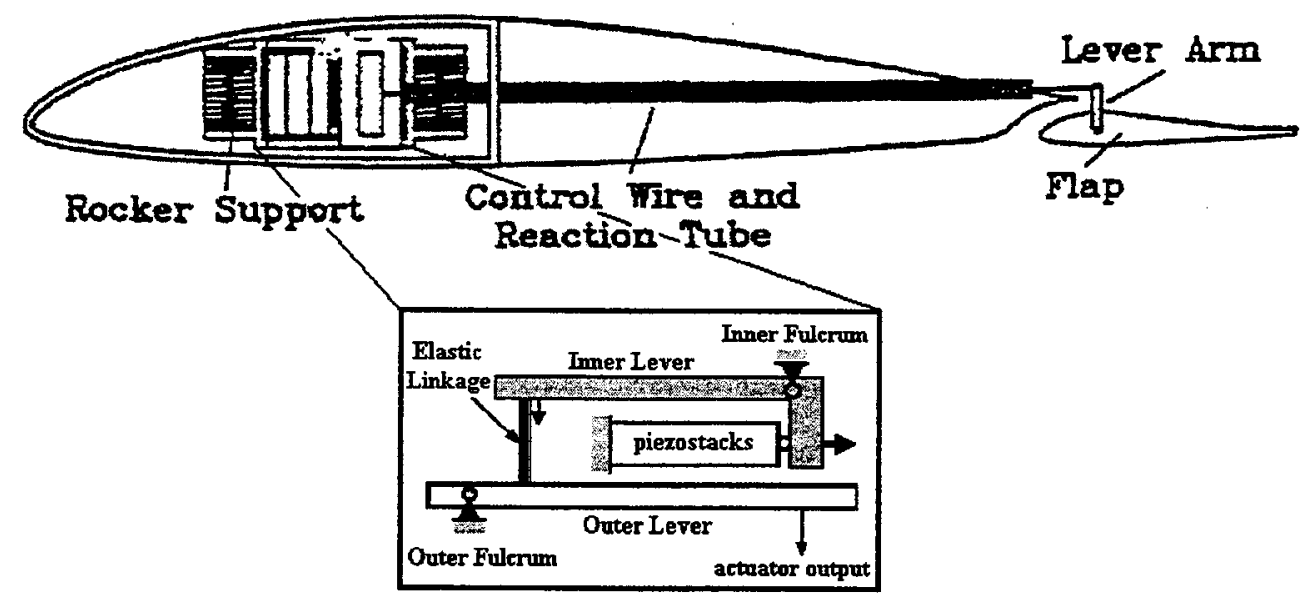

Figure 1.3: Blade with trailing edge flap and piezostack actuators

edge flaps the reader is referred to the works of Straub et al [8], Lee and Chopra [6], and Straub [9].

\section{Smart Spring}

The smart spring also incorporates piezoelectric stacks as outlined in Nitzsche et al [10]. In this concept, a stack of piezoelectrics located somewhere along the span, often at or close to the blade root as in Figure 1.4, would upon actuation become an integral part of the blade structure. This actuation thus changes the structural stiffness and dynamic characteristics of the rotor blade thereby affecting its dynamic behaviour. This concept has been developed by Nitzsche et al in [11, 12], and was also investigated by [13] at Carleton University under a contract with the National Research Council of Canada (NRC), Institute for Aerospace Research. The initial investigations show promising results but the work is ongoing. Further analyses in stability were carried out by Morozova [14]. 


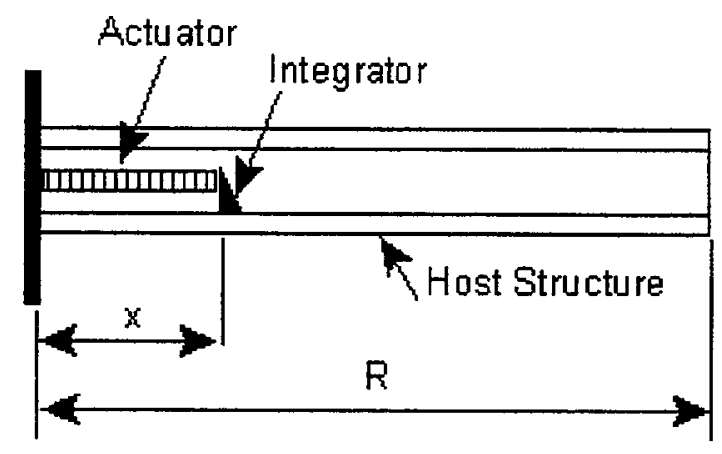

Figure 1.4: Side view of the smart spring blade
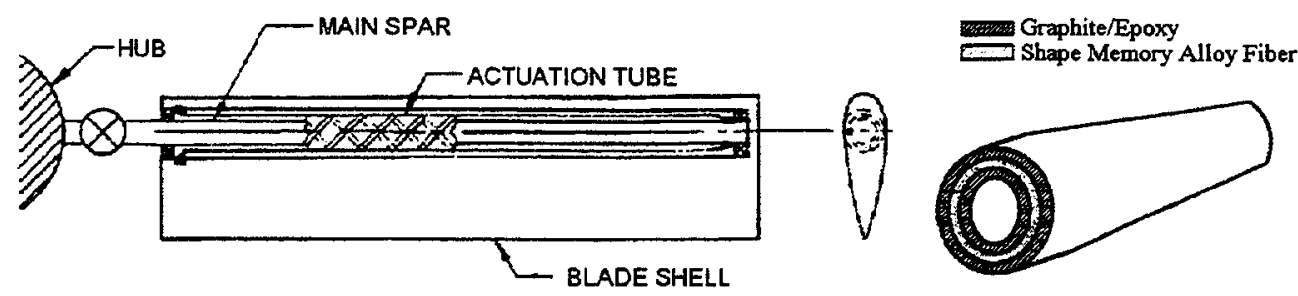

Figure 1.5: Pitch control by use of SMA torque tube

\section{SMA Torque Tubes}

Blade twist is achieved in this method by transferring the torque of a long tube to the spar section. Among the few variations, one uses a short section of shape memory alloy (SMA) material at the root of the blade, attached to a long passive torque tube that runs the span of the blade. This application takes advantage of the high strain capabilities of SMAs in transferring torque to the tube when the SMAs are actuated. The stiff properties of the tube provide sufficient authority to change the angle of incidence of blade by the same increment along the span. Figure 1.5 illustrates one example of the SMA torque tube [9]. This application has been investigated by Jacot [7], Straub [9], Narkiewicz [15], and Strehlow and Rapp [16]. 


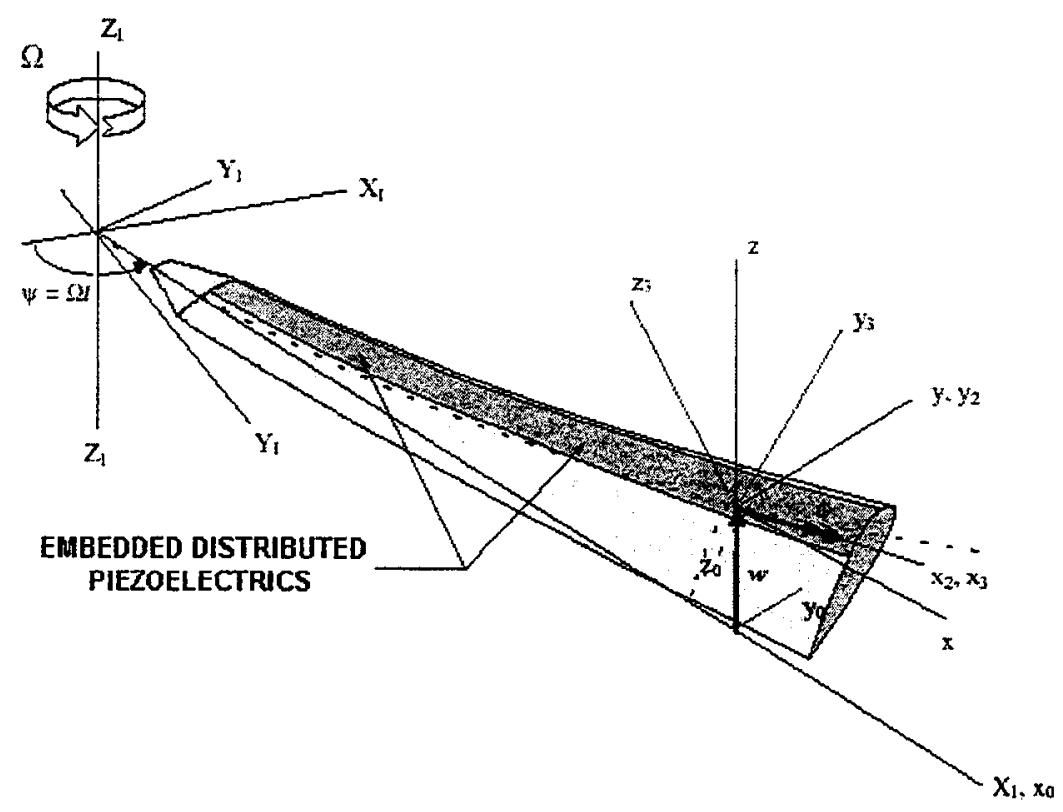

Figure 1.6: Induced twist from piezoelectric actuation

\section{Embedded Piezoelectrics}

This final approach, the one that is applied in this thesis, uses embedded piezoelectrics along the blade span or portions thereof to suppress vibrations. It is also one of the most common methods for vibration suppression that is currently under investigation. This type of individual blade control uses the direct shear stresses produced from the anisotropic actuation of the piezoelectric material to induce twist. Figure 1.6 [17] illustrates that by using distributed piezoelectric elements along the blade span, and an angled orientation of the composite material from the elastic axis, twisting will result. The advantages that piezoelectric materials provide for blade applications are largely in the conformability and tailorability of the material. Piezoelectric materials also provide high levels of actuation authority due to their high Young's modulus values. Therefore the $\pm 3^{\circ}$ of twist needed by Shaw and Albion may be achieved. What is also attractive for this type of IBC is the high bandwidth associated with piezoelectrics that the HHC approach lacks. 
Experimental work with piezoelectrics bonded onto composites structures and cantilevered beams began with the work of Crawley and de Luis [18], and later for anisotropic and isotropic plates by Crawley and Lazarus [19]. A preliminary analytical model based on classical plate theory was used for predicting behaviour. At the same time, a passive beam structural model was developed by Rehfield [20], and Rehfield and Atilgan [21] to model one-dimensional single-celled composite blades. It was in the work of Barrett [22] that methods of directional attachment for bonding piezoelectric elements to plates and aerodynamic surfaces was established.

The first scaled model of a rotor blade, manufactured for the purposes of experimenting with induced strain actuation from piezoelectric elements, was accomplished by Chen and Chopra $[23,24]$. A 1/8th Froude-scaled blade model was built with embedded monolithic piezoceramic elements at $\pm 45^{\circ}$. These monolithic ceramic materials take advantage of the shear lag effect to induce shear stresses that cause blade twist. The blade was tested to validate theory, for variations in bond thickness, actuator skew angle and spacing, but only 0.1 degrees of twist was achieved for a goal of 2 degrees. Later, in a series of hover tests, 0.5 degrees of twist was achieved by this group at the University of Maryland [25].

However, since these piezoceramic wafers do not produce direct anisotropic actuation, but exhibit in-plane isotropy and use the composite coupling effects to produce twist, the Piezoelectric Fibre Composite (PFC) was introduced by Bent $[26]^{1}$. Much of the work that has since surrounded applications in embedded piezoelectric actuation for vibration suppression has focussed on applying this material.

\footnotetext{
${ }^{1}$ The Piezoelectric Fibre Composite (PFC) was the initial development of this technology-driven design. Later, as the interdigitated electrode pattern was implemented and other researchers utilized the approach as well, the PFC became generally known as the active fibre composite (AFC). PFC and AFC will be used interchangeably in this thesis.
} 


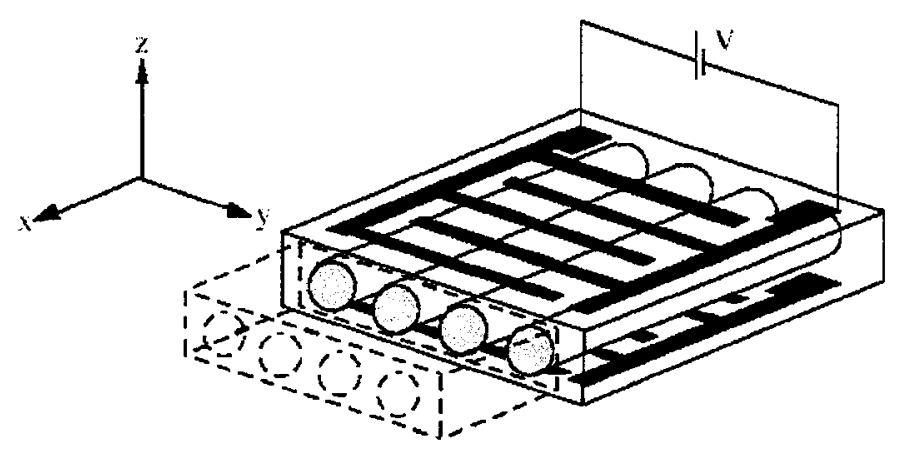

Figure 1.7: Active fibre composite with interdigitated surface electrode pattern

\subsubsection{Active Fibre Composites}

Active fibre composites are described as "uniaxially aligned piezoelectric fibres (e.g. PZT) embedded in a polymer matrix between two interdigitated surface electrodes as the driving voltage supply" [27]. Figure 1.7 illustrates the AFC configuration for a single ply [28]. The electric field from the interdigitated electrodes in the through-thickness or z-direction causes the piezoelectric fibres to strain in the longitudinal plane or x-direction. The stiffness of the composite is therefore a combination of the fibre stiffness in the longitudinal direction, and the transverse stiffness caused by the matrix.

Piezoceramic wafers, on the other hand, produce straining in the direction of the applied electric field - the z-direction, with half the amount of strain produced in the transverse direction. Therefore AFCs are shown to have the advantage over the monolithic wafers in producing direct planar anisotropic shear stress. As a result AFCs provide greater conformability to an airfoil surface, increased tailorability, and enhanced performance.

The preliminary work for experimenting with the induced twist from the AFC was performed by Bent et al [29], and Rodgers and Hagood [30] at MIT's Active Materials and Structures Laboratory. The manufacturing process for embedding the piezoelectric fibres in the epoxy matrix was established and testing was performed for composite plates. Testing was expanded to include a bench test for a 1/16th Froude-scale model of the 
Chinook CH-47D helicopter blade in the work by du Plessis [28]. Static tip deflections were on the order of $1.4^{\circ}$, affirming the actuation authority that is possible with AFCs. The analytical model used for the basis of the testing was built on the single-cell formulation of Rehfield - termed the Rehfield model. Derham and Hagood [31] utilized the results of these previous researchers to present a cost-benefit analysis that illustrated the impact of using this technology. This paper also introduced a 1/6th Mach-scaled CH-47D blade that was being tested for hover flight by Rodgers and Hagood [32, 33]. This particular test experimented with the effects of rotor speed, actuation frequencies, and blade loading conditions for hover flight, on induced twist. An aeroelastic analysis was presented by Wilkie [17] for a thin-walled one-celled box beam section. Similar to du Plessis, this work was based on the Rehfield model, and achieved between one to two degrees of tip twist.

The Rehfield model, however, was shown to have limitations by Berdichevkskii [34] and Badir [35]. The model is limited to single-celled beams and is not, in fact, asymptotically correct. Thus a new analytical formulation for multi-celled beams was derived by Badir, which formulates the stiffness constants for a cross-section in an asymptotically correct fashion. This cross-sectional formulation was implemented by Cesnik and Shin [36], Shin [37], and Bailey [38], however the one-dimensional modelling process varies between the two approaches. The work of Cesnik and Shin utilises the exact intrinsic equations of motion for rotating beams - considering small strains and finite rotations - formulated by Shang and Hodges [39]. The one-dimensional beam formulation that was used by Bailey is based on the dynamic equations of motion for a rotor blade in hover, given by Hodges and Dowell [40], and Hodges and Ormiston [41].

This thesis focusses on implementing the asymptotically correct cross-sectional analysis of Badir with the dynamic formulation for the one-dimensional beam structure in hover of Hodges and Ormiston, and Hodges and Dowell, while improving the model that was used by Bailey. 


\subsection{Thesis Overview}

Chapter 1 serves to introduce the vibration problem that plagues today's helicopters. It discusses the long-standing issues that provide motivation for producing a better, performance-enhanced, and generally smoother-running helicopter. Amongst the various methods for addressing the vibration problem, embedded distributed piezoelectrics show promising results for being able to suppress these vibrations. This kind of smart technology in the form of active fibre composites, achieves sufficient authority to twist a rotor blade to dampen vibrations in a stable manner.

Chapter 2 outlines the methodology used to obtain both the cross-sectional stiffness properties and the terms that originate from the active contribution in order to describe the smart rotor blade. The analysis is applied to a two-celled, thin-walled, anisotropic composite rotor section. It is based on Badir's variational asymptotic method, but is modified to include the contributions from piezoelectric strain as suggested by Cesnik and Shin.

Chapter 3 builds on the nonlinear beam formulation that Hodges and Dowell, and Hodges and Ormiston used to describe rotor blade deformation of a helicopter in hover. Blade deformation in the lead-lag, flap and torsional sense is derived from the equations of motion for a rotating cantilever beam. However this formulation is adjusted to take into account the stiffness and active contributions defined by the cross-sectional analysis of Chapter 2. Structural, kinetic, and aerodynamic contributions are brought together into Hamilton's equation and solved numerically using Galerkin's method. The resulting ordinary differential equations are solved for their steady-state and perturbation parts.

Chapter 4 describes the procedure for designing and developing an analytical blade model. This involves factoring in several design considerations for a composite, active, blade such as ply orientation or percent of blade span to be actuated. To ensure that typical 
characteristics of a blade are met, the proposed designs are modelled on the structural (natural frequencies) and dynamic (Lock number) properties of a scaled Eurocopter BO105 rotor. Several candidates are examined and three final designs are chosen, having satisfied the stiffness requirements. A validation study is also carried out to compare the stiffness constants with published results for an arbitrary box beam blade section.

Chapter 5 presents the stability results for the three final designs. Successful designs exhibit negative-valued natural frequencies for certain vibrational modes; this signifies no tendencies towards the aeroelastic instabilities that are divergence and flutter. Besides the passive blade that these designs are modelled on, the blades are tested for zero actuation, actuation under a DC current, and actuation with an AC current. This chapter also includes a validation study against published results for the calculation of the natural frequencies. Lastly, this chapter introduces the control law for the blade by signalling an impulsive actuation and observing the response.

Chapter 6 summarizes the achievements made in this thesis. Several conclusions are drawn from the results that have contributed to the modelling and stability analysis of the smart hingeless rotor blade in hover. Finally, recommendations are made in order to provide a guideline for future work and experimental testing in this area. 


\section{Chapter 2}

\section{Cross-Sectional Analysis}

This chapter discusses the analyses that make up the structural formulation behind the final dynamic equations of rotor blade motion. The structural modelling is composed of two fundamental parts: a linear 2D cross-sectional analysis that is presented in this chapter and a 1D beam formulation as outlined in the following chapter. This crosssectional analysis provides the characteristic properties of the rotor blade for stiffness and contributions due to actuation.

This cross-sectional analysis is based on the variational asymptotic method which was originally developed by Berdichevskii [34]. This work was further expanded by Badir to include multi-celled anisotropic composite beams [35, 42]. The variational asymptotic method is applied to thin-walled beams under extension, torsion, and bending and utilises 2D shell energy relations. The outcome of the process produces the cross-sectional stiffness constants that can be used in the dynamic modelling of the rotor blade motion. In addition to the stiffness constants, the closed-form expressions for displacements and stress fields are also found. Integral actuation was introduced to the model by Cesnik and Shin [36] by including the active fibre composite in the cross-sectional analysis.

This 2D model, while expressed as a linear formulation, accurately represents the two- 
celled, thin-walled cross-section under deformation desired by the helicopter blade model. It accurately represents the actual blade shape, and to its further advantage is flexible in defining the material properties and number of cells desired. Furthermore, the warping deformation that previous theories had struggled with [21, 43, 44, 45], is addressed in a satisfactory manner by Badir in this model.

This chapter will not only provide a short review of the work accomplished by Badir, Cesnik and Shin, but also will present some minor changes. It also serves to provide the background needed for the next section on the 1D beam formulation. A detailed account may be found in Badir [35], Cesnik and Shin [36], and Bailey [38].

\subsection{Reference Frame}

Consider a slender thin-walled elastic cylindrical shell as shown in Figure 2.1 which is assumed to represent a typical helicopter blade. The following is assumed

$$
\frac{d}{L} \ll 1, \frac{h}{d} \ll 1, \frac{h}{R} \ll 1
$$

where length of the blade is represented by $L$, thickness by $h$, radius of curvature of the middle surface by $R$, and the maximum cross-sectional dimension by $d$. Curvilinear coordinates are represented by $\xi, s$, and $x$, in the normal, tangential, and longitudinal directions

such that $\xi$ is bounded by $-\frac{h(s)}{2}<\xi<\frac{h(s)}{2}$. The cross-sectional twist experienced by the blade is given by $\varphi$.

The $x$-axis in Figure 2.1 represents the elastic axis of the blade. The Cartesian coordinates $x, y, z$ of any point on the middle surface around the cross-section can be expressed in terms of curvilinear coordinates $\xi, s$, and $r$. In addition, the tangential vector $\mathbf{t}$, the normal vector $\mathbf{n}$, and the projection of the position vector $\mathbf{r}$ on $\mathbf{t}$ and $\mathbf{n}$ are expressed in 


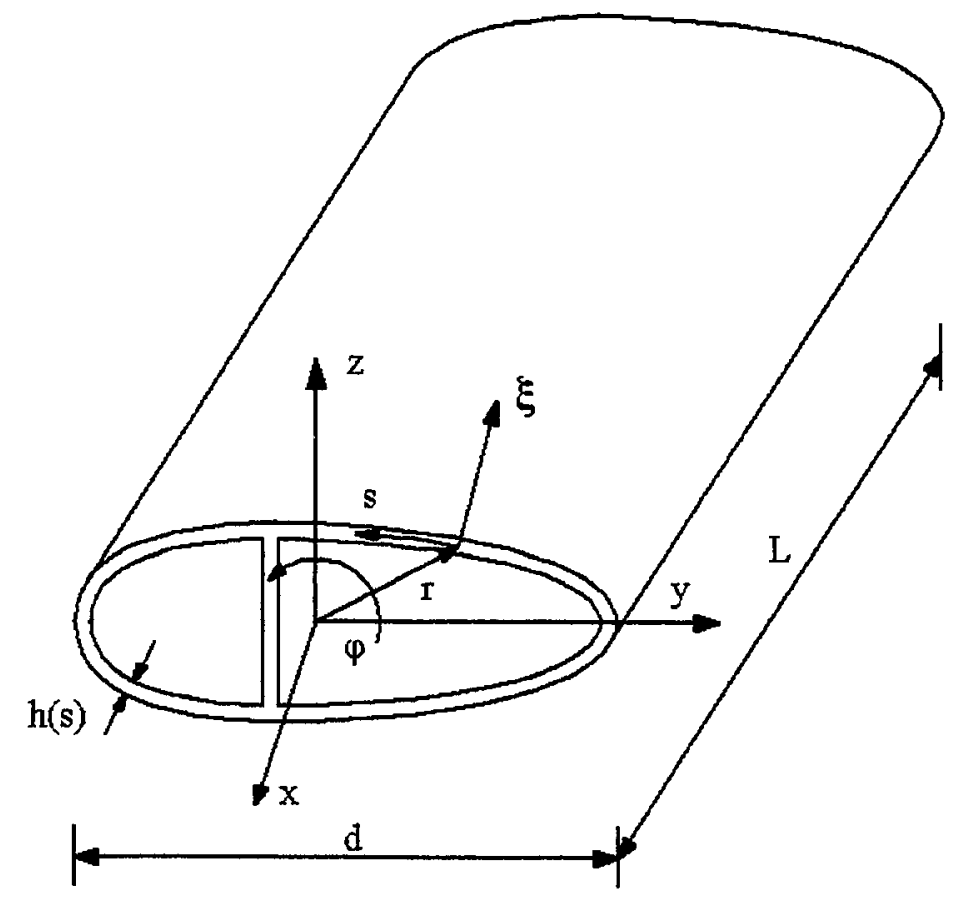

Figure 2.1: Two-celled thin-walled blade cross-section

the Cartesian and curvilinear coordinates as

$$
\begin{aligned}
\mathbf{t} & =\frac{d \mathbf{r}}{d s}=\frac{d y}{d s} \mathbf{i}_{y}+\frac{d z}{d s} \mathbf{i}_{z} \\
\mathbf{n} & =\mathbf{t} \times \mathbf{i}_{x}=\frac{d z}{d s} \mathbf{i}_{y}-\frac{d y}{d s} \mathbf{i}_{z} \\
r_{t} & =\mathbf{r} \cdot \mathbf{t}=y \frac{d y}{d s}+z \frac{d z}{d s} \\
r_{n} & =\mathbf{r} \cdot \mathbf{n}=y \frac{d z}{d s}-z \frac{d y}{d s}
\end{aligned}
$$

where $\mathbf{i}_{x}, \mathbf{i}_{y}$, and $\mathbf{i}_{z}$ are the unit vectors in the $x, y$, and $z$ directions.

The 2D cross-section in Figure 2.1, [35], may be considered to be situated at a point $x$ along the blade span. Hodges and Ormiston represent the blade frame of reference at a larger scale for both global $X, Y, Z$ and local $x, y, z$ coordinate systems, Figure 2.2, [41]. The local coordinate system of Hodges and Ormiston will be related to the Cartesian 


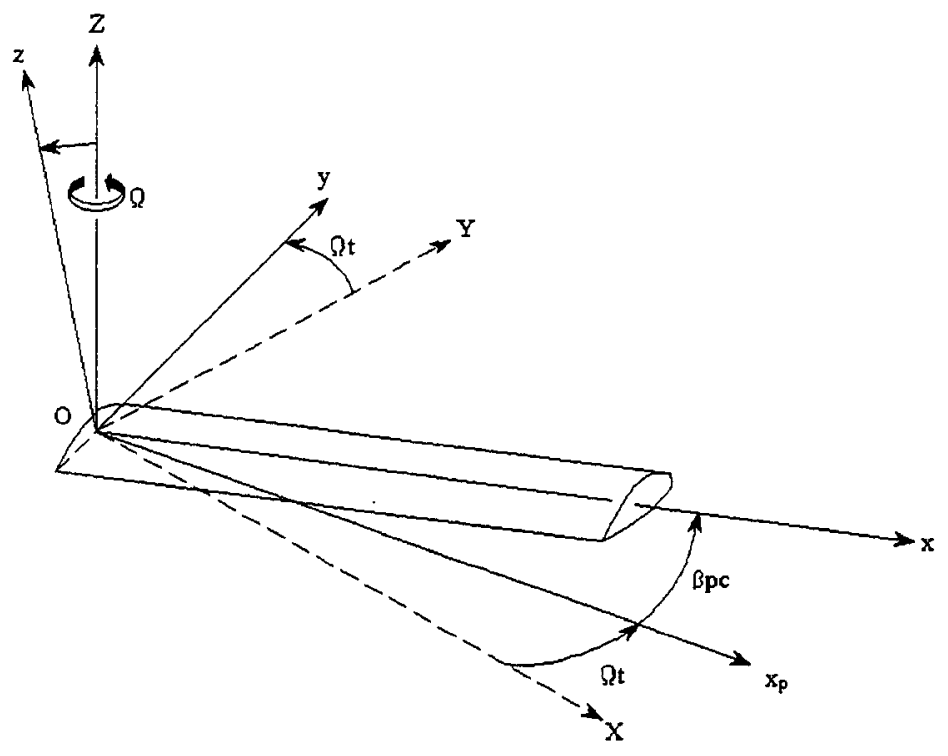

Figure 2.2: Blade frames as derived by Hodges and Ormiston

frame of Badir in Chapter 3 in the meshing of the 2D linear formulation with the $1 \mathrm{D}$ nonlinear beam formulation.

\subsection{Shell Energy Functional}

The foundation of the variational asymptotic approach begins with the energy density analysis of an elastic body. This involves an iterative process, which at the beginning of each successive stage correction factors are added to the displacement functions, and at the end of the stage the energy functional is assessed for continuity of order. The analysis begins with the zeroth-order approximation where the displacement functions are obtained by retaining the leading order terms in the energy functional. The iterative process terminates when the addition of any new higher order terms to the energy functional are deemed to be negligible. 
To begin, the strain energy density of a 3D elastic body is given by

$$
U=\frac{1}{2} E^{i j k l} \varepsilon_{i j}^{(m)} \varepsilon_{k l}^{(m)}
$$

where $i, j, k, l=1,2,3$. The $3 \mathrm{D}$ Hookean tensor by which the material properties are defined is denoted by $E^{i j k l}$, and the mechanical strain is given by $\varepsilon^{(m)}$. It is noted that for an active fibre composite the total strain, $\varepsilon_{i j}$, is comprised of the mechanical strain, $\varepsilon^{(m)}$, as well as the non-mechanical strain, $\varepsilon^{(n m)}$, one has

$$
\varepsilon_{i j}=\varepsilon_{i j}^{(m)}+\varepsilon_{i j}^{(n m)}=\varepsilon_{i j}^{(m)}+\varepsilon_{i j}^{(a)}
$$

where $a$ represents actuation strain.

Moreover, the expression for total strain can be written as a function of the piezoelectric actuation stemming from the applied electric field

$$
\varepsilon_{i j}=s_{i j k l}^{E} \sigma_{k l}+d_{k i j} E_{k}
$$

where $s_{i j k l}^{E}$ is the elastic compliance tensor at constant electric field, $E_{k}, \sigma_{k l}$ represents the stress tensor, and $d_{k i j}$ is the piezoelectric electromechanical coupling tensor. The total strain can also be represented in terms of $2 \mathrm{D}$ strain measures as

$$
\varepsilon_{i j}=\gamma_{i j}+\xi \rho_{i j}
$$

where $\gamma_{i j}$ and $\rho_{i j}$ represent the in-plane strain components and the change in shell middle surface curvature, respectively. For a cylindrical shell [46], these strain components are 
related to the corresponding displacements by

$$
\begin{aligned}
\gamma_{11} & =\frac{\partial v_{l}}{\partial x} \\
2 \gamma_{12} & =\frac{\partial v_{l}}{\partial s}+\frac{\partial v_{s}}{\partial x} \\
\gamma_{22} & =\frac{\partial v_{s}}{\partial s}+\frac{v_{\xi}}{R} \\
\rho_{11} & =\frac{\partial^{2} v_{\xi}}{\partial x^{2}} \\
\rho_{12} & =\frac{\partial^{2} v_{\xi}}{\partial x \partial s}+\frac{1}{4 R}\left(\frac{\partial v_{l}}{\partial s}-3 \frac{\partial v_{s}}{\partial x}\right) \\
\rho_{22} & =\frac{\partial^{2} v_{\xi}}{\partial s^{2}}-\frac{\partial}{\partial s}\left(\frac{v_{s}}{R}\right)
\end{aligned}
$$

where $v_{l}, v_{s}$, and $v_{\xi}$ represent the middle surface displacements in the axial, tangential, and normal directions respectively. These middle surface displacements are further related to the corresponding Cartesian displacement components $u_{x}, u_{y}$, and $u_{z}$ by

$$
\begin{aligned}
v_{l} & =u_{x} \\
v_{s} & =u_{y} \frac{d y}{d s}+u_{z} \frac{d z}{d s} \\
v_{\xi} & =u_{y} \frac{d z}{d s}-u_{z} \frac{d y}{d s}
\end{aligned}
$$

From classical theory of thin shells it can be assumed that the through-thickness stresses $\sigma_{i 3}$ for $i=1,2,3$, are considerably smaller than the other stress components $\sigma_{i j}$. The 3D strain energy equation can then be minimized with respect to the corresponding strains, $\varepsilon_{i 3}$, thus reducing the equation from three dimensions to two. The resulting $2 \mathrm{D}$ strain energy is

$$
\hat{U}=\left.\min U\right|_{\varepsilon_{i 3}}=\frac{1}{2} D^{\alpha \beta \gamma \delta} \varepsilon_{\alpha \beta}^{(m)} \varepsilon_{\gamma \delta}^{(m)}
$$

where $D^{\alpha \beta \gamma \delta}$ is the $2 \mathrm{D}$ Hookean tensor for material properties, and $\alpha, \beta, \gamma, \delta=1,2$.

Further details of the derivation of the 2D Hookean tensor may be found in Bailey, but 
the following equation describes it as

$$
D^{\alpha \beta \gamma \delta}=E^{\alpha \beta \gamma \delta}-\frac{E^{\alpha \beta 33} E^{\gamma \delta 33}}{E^{3333}}-H_{\mu \lambda} G^{\alpha \beta \mu} G^{\gamma \delta \lambda}
$$

where

$$
G^{\alpha \beta \mu}=E^{\alpha \beta \mu 3}-\frac{E^{\alpha \beta 33} E^{\mu 333}}{E^{3333}}
$$

and $H_{\mu \lambda}$ are the components of the inverse of the $2 \mathrm{D}$ matrix

$$
\left\|E^{\mu 3 \lambda 3}-\frac{E^{\mu 333} E^{\lambda 333}}{E^{3333}}\right\|
$$

From the standpoint of the piezoelectric fibres, only the electric field running parallel to the fibre direction needs to be taken into consideration, the corresponding field is denoted by $E_{e}$. Hence, the 2D strain expression from Eqs. (2.5) and (2.6) reads as

$$
\begin{aligned}
\varepsilon_{\alpha \beta}^{(m)} & =\varepsilon_{\alpha \beta}-\varepsilon_{\alpha \beta}^{(a)} \\
& =\gamma_{\alpha \beta}+\xi \rho_{\alpha \beta}-d_{e \alpha \beta} E_{e}
\end{aligned}
$$

The shell energy per unit middle surface area, $\Phi$, may then be determined through substitution of Eq. (2.10) into $\hat{U}$ and subsequent integration over the thickness, $\xi$, i.e.,

$$
\begin{aligned}
2 \Phi= & \left(<D^{\alpha \beta \gamma \delta}>\gamma_{\alpha \beta}-2<D^{\alpha \beta \gamma \delta} d_{e \alpha \beta} E_{e}>\right) \gamma_{\gamma \delta} \\
& +2\left(<D^{\alpha \beta \gamma \delta} \xi>\gamma_{\alpha \beta}-<D^{\alpha \beta \gamma \delta} d_{e \alpha \beta} E_{e} \xi>\right) \rho_{\gamma \delta} \\
& +<D^{\alpha \beta \gamma \delta} \xi^{2}>\rho_{\alpha \beta} \rho_{\gamma \delta}+<D^{\alpha \beta \gamma \delta} d_{e \alpha \beta} d_{e \gamma \delta} E_{e}^{2}>
\end{aligned}
$$


where the integrals above are defined by

$$
<(\bullet)>=\int_{-h(s) / 2}^{+h(s) / 2}(\bullet) d \xi
$$

The final term in Eq. (2.11), representing the quadratic terms in the electric field, will have no influence on the variational derivation of beam stiffness and actuation constants since the actuation field is considered to be prescribed.

Recall that the potential energy of any system $\Pi$ is given as

$$
\Pi=U+\Omega
$$

where $U$ is strain energy and $\Omega$ is the potential of the applied load. For the current application, given that the applied external load is represented by $P_{i}$, the displacement field is $u_{i}$, and the potential energy is $I$, the above equation may be rewritten such that

$$
I=\iint \Phi d x d s-\iint P_{i} u_{i} d x d s
$$

\subsubsection{Ordering Scheme}

At this point in the 2D linear cross-sectional formulation it is necessary to outline the ordering scheme carried throughout the variational asymptotic analysis. Following Badir's approach, terms of order $O>\varepsilon^{2}$ are neglected where $\varepsilon$ is assumed to be the order of the following variables,

$$
\frac{d}{L} \sim O(\varepsilon), \frac{h}{d} \sim O(\varepsilon), \frac{h}{R} \sim O(\varepsilon)
$$

Further variables whose magnitudes are assumed to obey an ordering scheme are given in Table 2.1.

Moreover it is assumed that the radius of curvature, $R$ and the displacements $v_{l}, v_{s}$ and 
Table 2.1: Major ordering variables used in displacement functions

\begin{tabular}{|c|c|c|}
\hline definition & variable & order \\
\hline curvilinear displacements & $v_{s}, v_{\xi}$ & $\Delta$ \\
curvilinear axial displacement & $v_{l}$ & $\frac{\Delta d}{L}$ \\
stiffness coefficients & $D^{\alpha \beta \gamma \delta}, E^{i j k l}$ & $E$ \\
axial coordinates & $x$ & $L$ \\
circumferential coordinates & $s$ & $d$ \\
normal coordinates & $\xi$ & $h$ \\
\hline
\end{tabular}

$v_{\xi}$ are much less than the length of the blade $L$. As well, $d$ may be considered smaller or of the same order as $R$. This means that for the thin-walled slender beam whose dimensions satisfy Eq. (2.1), the rate of change of displacement in the axial direction is considerably smaller than the rate of change in the circumferential direction, i.e.,

$$
\left\|\frac{\partial v_{i}}{\partial x}\right\| \ll\left\|\frac{\partial v_{i}}{\partial s}\right\|
$$

Appendix A.1 lists the results of the orders for the significant terms that follow in the cross-sectional analysis.

\subsection{Variational Asymptotic Analysis}

As stated above, the variational asymptotic analysis requires several iterations before an approximate solution can be reached. It begins with the zeroth-order approximation where, using the ordering scheme discussed above, various strain and curvature terms can be ignored along with the contribution of external forces in the energy functional given in Eq. (2.13). Recalling that the deformed state of the beam is represented by the stationary point of the energy functional, the displacement field associated with the zeroth order 
approximation is determined to be:

$$
\begin{aligned}
& v_{l}=U_{1}(x) \\
& v_{s}=U_{2}(x) \frac{d y}{d s}+U_{3}(x) \frac{d z}{d s}+\varphi(x) r_{n} \\
& v_{\xi}=U_{2}(x) \frac{d z}{d s}-U_{3}(x) \frac{d y}{d s}-\varphi(x) r_{t}
\end{aligned}
$$

where $U_{1}, U_{2}, U_{3}$ represent the average cross-sectional translation and $\varphi(x)$ represents the torsional rotation. The first order approximation is achieved by adding correction warping factors $w_{1}, w_{2}$, and $w_{3}$ respectively to the $v_{l}, v_{s}$, and $v_{\xi}$ expressions of Eq. (2.15). Due to absence of any pressure difference between the interior and exterior of the shell, the hoop strain will be minimal compared to other strains. Hence, the shell energy per unit middle surface area can be minimized with respect to the hoop strain. The resulting expression for shell energy whose strains are now expressed by the displacements and warping terms of Eq. (2.15), now reveals

$$
2 \Phi=\left(A \gamma_{11}-2 A^{(a)}\right) \gamma_{11}+2 B \gamma_{11} \gamma_{12}+\left(C \gamma_{12}-2 C^{(a)}\right) \gamma_{12}-\Psi_{2}\left(E_{e}\right)
$$

where the axial, coupling, and shear stiffness are represented by $A, B$, and $C$ respectively. These are the terms that may be considered to be analogous to classical laminate stiffness $A, B$, and $D[47,48,49]$. The variables $A^{(a)}, C^{(a)}$, and $\Psi_{2}\left(E_{e}\right)$ are the contributions to the stiffness from actuation as follows:

$$
\begin{aligned}
A & =\left\langle D^{111}>-\frac{\left.<D^{1122}\right\rangle^{2}}{\left.<D^{2222}\right\rangle}\right. \\
B & =2<D^{1112}>-2 \frac{\left\langle D^{1122}><D^{1222}\right\rangle}{\left.<D^{2222}\right\rangle} \\
C & =4<D^{1212}>-4 \frac{\left\langle D^{1222}\right\rangle^{2}}{\left.<D^{2222}\right\rangle} \\
A^{(a)} & \left.=<D^{\alpha \beta 11} d_{e \alpha \beta} E_{e}>-\frac{\left\langle D^{1122}\right\rangle}{\left.<D^{2222}\right\rangle}<D^{\alpha \beta 22} d_{e \alpha \beta} E_{e}\right\rangle
\end{aligned}
$$




$$
\begin{aligned}
C^{(a)} & =2<D^{\alpha \beta 12} d_{e \alpha \beta} E_{e}>-2 \frac{<D^{1222}>}{<D^{2222}>}<D^{\alpha \beta 22} d_{e \alpha \beta} E_{e}> \\
\Psi_{2}\left(E_{e}\right) & =\frac{\left.<D^{\alpha \beta 22} d_{e \alpha \beta} E_{e}\right\rangle^{2}}{<D^{2222}>}
\end{aligned}
$$

To minimize the corresponding energy functional, $I$, its first variation $\delta I$ is set to zero. This gives rise to the resultants of the axial stress $N_{11}$, shear flow $N_{12}$, and hoop stress $N_{22}$, where

$$
\begin{aligned}
N_{11} & =\frac{\partial \Phi}{\partial\left(\gamma_{11}\right)}=\left(A(s) \gamma_{11}+B(s) \gamma_{12}\right)-A^{(a)}(s) \\
N_{12} & =\frac{\partial \Phi}{\partial\left(2 \gamma_{12}\right)}=\frac{1}{2}\left(B(s) \gamma_{11}+C(s) \gamma_{12}\right)-\frac{1}{2} C^{(a)}(s) \\
& =\text { constant } \\
N_{22} & =\frac{\partial \Phi}{\partial\left(2 \gamma_{22}\right)} \\
& =0
\end{aligned}
$$

which is similar to the classical solution of vanishing hoop stress resultant and constant shear flow in thin-walled beams. Considering $\delta I$, it can be concluded that since $N_{22}=0$, the variations of $\delta w_{2}$ and $\delta w_{3}$ are inconsequential and hence the energy functional is independent of $w_{2}$ and $w_{3}$ and the only warping term that needs to be determined is $w_{1}$. Thus one may consider the expression for $N_{12}$ in Eq. (2.18). Arising from corresponding shear strains, the correction factor $w_{1}$ for the $i$ th section can be found from

$$
\begin{aligned}
\frac{\partial w_{1}}{\partial s} & =\frac{4}{C} q_{i}-2 \frac{B}{C} U_{1}^{\prime}+\frac{2}{C} C^{(a)}-U_{2}^{\prime} \frac{d y}{d s}-U_{3}^{\prime} \frac{d z}{d s}-\varphi^{\prime} r_{n} \\
& =-2 c(s) q_{i}+b(s) U_{1}^{\prime}-c(s) C^{(a)}-U_{2}^{\prime} \frac{d y}{d s}-U_{3}^{\prime} \frac{d z}{d s}-\varphi^{\prime} r_{n}
\end{aligned}
$$

where $q_{i}$ represents the shear flow in the $i$ th branch, and $c(s)$ and $b(s)$ are

$$
c(s)=-2 \frac{1}{C}
$$




$$
b(s)=-2 \frac{B}{C}
$$

Letting $q_{1}, q_{2}$, and $q_{w}$ represent the shear flow in the left branch, right branch, and the web of a two-celled cross-section, and in view of constant shear flow established above, one has

$$
q_{w}=q_{1}-q_{2}
$$

Moreover, recalling that due to compatibility conditions for warping around each closed cell, one has

$$
\oint \frac{\partial w_{i}}{\partial s} d s=0
$$

where two compatibility equations for shear flow around each cell can subsequently be obtained. Along with these two equations, and Eq. (2.19), the shear flows $q_{1}, q_{2}$, and $q_{w}$ can be determined. Finally, integration of the expression in Eq. (2.19) will yield the expression for $w_{1}$. Then, the resulting displacement field after first order approximation reads,

$$
\begin{aligned}
v_{l}= & U_{1}(x)-y(s) U_{2}^{\prime}(x)-z(s) U_{3}^{\prime}(x) \\
& +G(s) \varphi^{\prime}(x)+g_{1}(s) U_{1}^{\prime}(x)+v_{1}^{(a)}(s) \\
v_{s}= & U_{2}(x) \frac{d y}{d s}+U_{3}(x) \frac{d z}{d s}+\varphi(x) r_{n} \\
v_{\xi}= & U_{2}(x) \frac{d z}{d s}-U_{3}(x) \frac{d y}{d s}-\varphi(x) r_{t}
\end{aligned}
$$

where $G(s), g_{1}(s)$, and $v_{1}^{(a)}(s)$ are defined in Appendix B.

By adding further warping correction factors $w_{4}, w_{5}$, and $w_{6}$ to the expressions of $v_{l}$, $v_{s}$, and $v_{\xi}$ respectively, of Eq. (2.22), a second-order approximation can be carried out in a manner similar to the one discussed for the 1st order. The resulting displacement field 
after such a second order approximation is

$$
\begin{aligned}
v_{l}= & U_{1}(x)-y(s) U_{2}^{\prime}(x)-z(s) U_{3}^{\prime}(x)+G(s) \varphi^{\prime}(x) \\
& +g_{1}(s) U_{1}^{\prime}(x)+g_{2}(s) U_{2}^{\prime \prime}(x)+g_{3}(s) U_{3}^{\prime \prime}(x)+v_{1}^{(a)}(s) \\
v_{s}= & U_{2}(x) \frac{d y}{d s}+U_{3}(x) \frac{d z}{d s}+\varphi(x) r_{n} \\
v_{\xi}= & U_{2}(x) \frac{d z}{d s}-U_{3}(x) \frac{d y}{d s}-\varphi(x) r_{t}
\end{aligned}
$$

where the variables $g_{2}(s)$ and $g_{3}(s)$ are given in Appendix B. The first four terms of $v_{l}$ in Eq. (2.23) are representative of those terms that would arise from a derivation using classical theory for extension, bending and torsion-related warping. The next three terms $g_{1}(s) U_{1}^{\prime}(x), g_{2}(s) U_{2}^{\prime \prime}(x)$, and $g_{3}(s) U_{3}^{\prime \prime}(x)$ arise from the derivation of warping due to axial strain and bending, and apply purely to an anisotropic material composition.

It should be noted that the original derivation by Badir for a single-celled anisotropic beam shows that Eq. (2.23) results in no explicit terms for transverse shear. This is due to the elimination of the hoop stress component $\sigma_{i 3}$ in Eq. (2.9) where transverse shear strains are implicitly expressed. This becomes important for multi-celled thin-walled beams, such as is the case here, when Eq. (2.21) requires implementation.

The strain fields associated with the second-order approximation are

$$
\begin{aligned}
\gamma_{11} & =U_{1}^{\prime}(x)-y(s) U_{2}^{\prime \prime}(x)-z(s) U_{3}^{\prime \prime}(x) \\
2 \gamma_{12} & =\left(\frac{d G}{d s}+r_{n}\right) \varphi^{\prime}(x)+\frac{d g_{1}}{d s} U_{1}^{\prime}(x)+\frac{d g_{2}}{d s} U_{2}^{\prime \prime}(x)+\frac{d g_{3}}{d s} U_{3}^{\prime \prime}(x)+\frac{d v^{(a)}(s)}{d s} \\
\gamma_{22} & =0
\end{aligned}
$$

This is the culmination of the terms needed to serve as a basis for forming the constitutive relations that are introduced in the next section. 


\subsection{Constitutive Relations}

The constitutive relations can be formed from known relationships between forces and moments from classical beam bending theory [46]. Axial force $F_{1}$, torsion $M_{1}$, and bending moments $M_{2}$ and $M_{3}$ are related to displacements, stresses, and stress resultants as

$$
\begin{aligned}
F_{1} & =\oint \frac{\partial \Phi}{\partial U_{1}^{\prime}} d s=\oint \int \sigma_{11} d \xi d s=\oint N_{11} d s \\
M_{1} & =\oint \frac{\partial \Phi}{\partial \varphi^{\prime}} d s=\oint \int \sigma_{12} r_{n}(s) d \xi d s=\oint N_{12} r_{n}(s) d s \\
M_{2} & =\oint \frac{\partial \Phi}{\partial\left(-U_{3}^{\prime \prime}\right)} d s=\oint \int \sigma_{11} z(s) d \xi d s=\oint N_{11} z(s) d s \\
M_{3} & =\oint \frac{\partial \Phi}{\partial U_{2}^{\prime \prime}} d s=-\oint \int \sigma_{11} y(s) d \xi d s=-\oint N_{11} y(s) d s
\end{aligned}
$$

Evaluation of each of the force and moments equations by separation of variables gives the constitutive relation in the form of stiffness constants, $K_{i j}$, and actuation forces and moments (distinguished by the superscript $(a)$ ), keeping in mind still that these relationships have been obtained while maintaining the order discussed in Section 2.2.1. This expression for an anisotropic two-celled actuated beam may be written in matrix form as

$$
\left\{\begin{array}{c}
F_{1} \\
M_{1} \\
M_{2} \\
M_{3}
\end{array}\right\}=\left[\begin{array}{llll}
K_{11} & K_{12} & K_{13} & K_{14} \\
K_{12} & K_{22} & K_{23} & K_{24} \\
K_{13} & K_{23} & K_{33} & K_{34} \\
K_{14} & K_{24} & K_{34} & K_{44}
\end{array}\right]\left\{\begin{array}{c}
U_{1}^{\prime} \\
\varphi^{\prime} \\
-U_{3}^{\prime \prime} \\
U_{2}^{\prime \prime}
\end{array}\right\}-\left\{\begin{array}{c}
F_{1}^{(a)} \\
M_{1}^{(a)} \\
M_{2}^{(a)} \\
M_{3}^{(a)}
\end{array}\right\}
$$

where detailed expressions for $F_{1}, M_{1}, M_{2}, M_{3}$, and $K_{i j}$ may be found also in Appendix B. The actuation force and moments provided by the piezoelectric fibres are lumped into one actuation vector shown above, whose corresponding expressions are provided as follows

$$
F_{1}^{(a)}=\oint\left(A^{(a)}-\frac{B}{C} C^{(a)}\right) d s+2 g_{1}^{(a)} \oint_{I} \frac{B}{C} d s+2 g_{2}^{(a)} \oint_{I I} \frac{B}{C} d s
$$




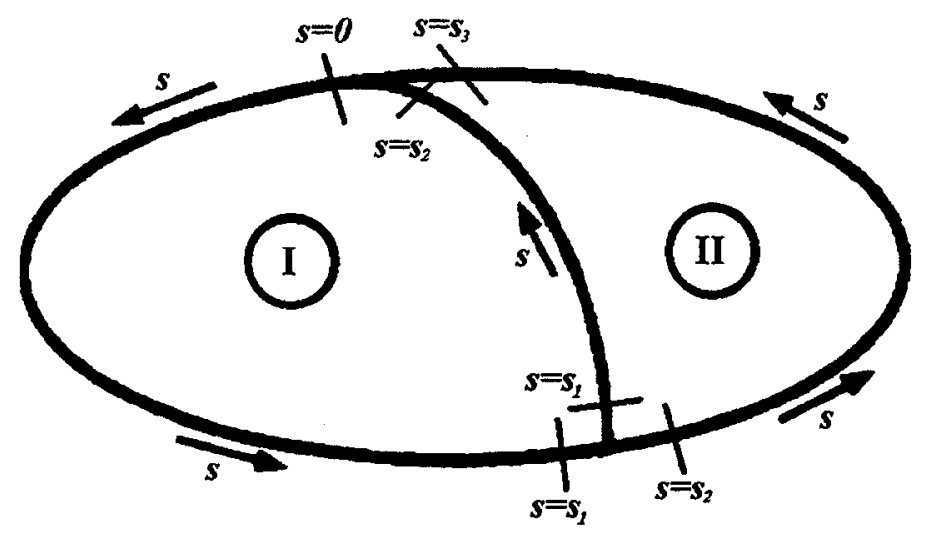

Figure 2.3: Branches for integration of a two-celled thin-walled cross-section

$$
\begin{aligned}
M_{1}^{(a)} & =2 g_{1}^{(a)} A_{e I}+2 g_{2}^{(a)} A_{e I I} \\
M_{2}^{(a)} & =\oint\left(A^{(a)}-\frac{B}{C} C^{(a)}\right) z d s+2 g_{1}^{(a)} \oint_{I} \frac{B}{C} z d s+2 g_{2}^{(a)} \oint_{I I} \frac{B}{C} z d s \\
M_{2}^{(a)} & =-\oint\left(A^{(a)}-\frac{B}{C} C^{(a)}\right) y d s-2 g_{1}^{(a)} \oint_{I} \frac{B}{C} y d s-2 g_{2}^{(a)} \oint_{I I} \frac{B}{C} y d s
\end{aligned}
$$

where Figure 2.3, [36], indicates the notation used in the various integrals of this chapter. The $\oint$ integral denotes evaluation from $0 \rightarrow s 1, s 1 \rightarrow s 2$, and $s 2 \rightarrow s 3$. Roman indices $I$ and $I I$ distinguish between integration of each cell. 


\section{Chapter 3}

\section{D Beam Analysis}

This chapter describes the second of the two formulations that must be accomplished in order to derive the dynamic equations of motion for an active helicopter blade, the first being the cross-sectional analysis and this, the 1D beam formulation. Two methods were carried out in order to obtain these equations. The first method - Method I - follows those coupled nonlinear equations first developed by Hodges and Dowell [40] and later completed by Hodges and Ormiston [41]. The second method - Method II - is a linear approximation that is based upon the strain energy approach by Bailey [38].

It was decided that Method I was a much more powerful way to express the dynamic equations of motion. Hodges and Ormiston, whose work expanded on previous linear approximations, show that in fact many linear analyses are limited for various reasons. Two important items may be curtailed in using a linear approach for articulated and hingeless helicopters: one, nonlinear structural or inertial coupling is important for determining the dynamic response, and two, in the determination of the aeroelastic stability of torsionally flexible blades. Other nonlinear terms include the coriolis and centrifugal inertial components, which without them one will loose accuracy in kinetic energy. However, as the ID beam formulation is a marriage of the components of Badir's structural formulation 
[35](by expanding on strain energy), Method II, with Hodges and Ormiston's nonlinear inertial and aerodynamic components, Method I, the author believes that it is valid, although restricted. As such, Method I will be described here, whereas Method II may be found in Appendix C.

As stated above, the nonlinear equations of motion developed for a smart blade are founded from Hodges and Ormiston and are executed using Hamilton's principle. The stiffness constants, $K_{i j}$, obtained from the variational structural analysis are used in place of Hodges and Ormiston's stiffness constants. Moreover, the actuation vector, affecting force and bending moment equations, is incorporated into Hodges and Ormiston's stress and moment resultants. The resulting integro-partial differential equations from Hamilton's principle are then written in nondimensional form. The application of Galerkin's method then expresses the effective blade dynamics into nonlinear, nonhomogeneous, constantcoefficient ordinary differential equations. Steady-state equilibrium quantities and unsteady perturbation quantities of the active blade are found through the application of either a $\mathrm{DC}$ or $\mathrm{AC}$ current to the distributed piezoelectrics. The relevant equations from Hodges and Ormiston's 1D beam formulation for obtaining the equations of motion are summarized here in order to incorporate the new actuation terms.

The blade is modelled as a long, straight, slender, cantilevered beam under moderate displacements. This formulation will apply to anisotropic composite beams. As an initial attempt, and in order to avoid modelling complexities arising from secondary configuration parameters such as droop, sweep, precone, pretwist, linear twist, and torque offset, as in Figure 3.1, [41], only precone and pretwist are to be considered. Moreover no offsets of mass, tension, and aerodynamic axes from the elastic axis are assumed. Finally, in simulating the effects of coupling between the inboard and outboard segments of the blade a coupling parameter, $\Re$ is introduced. 

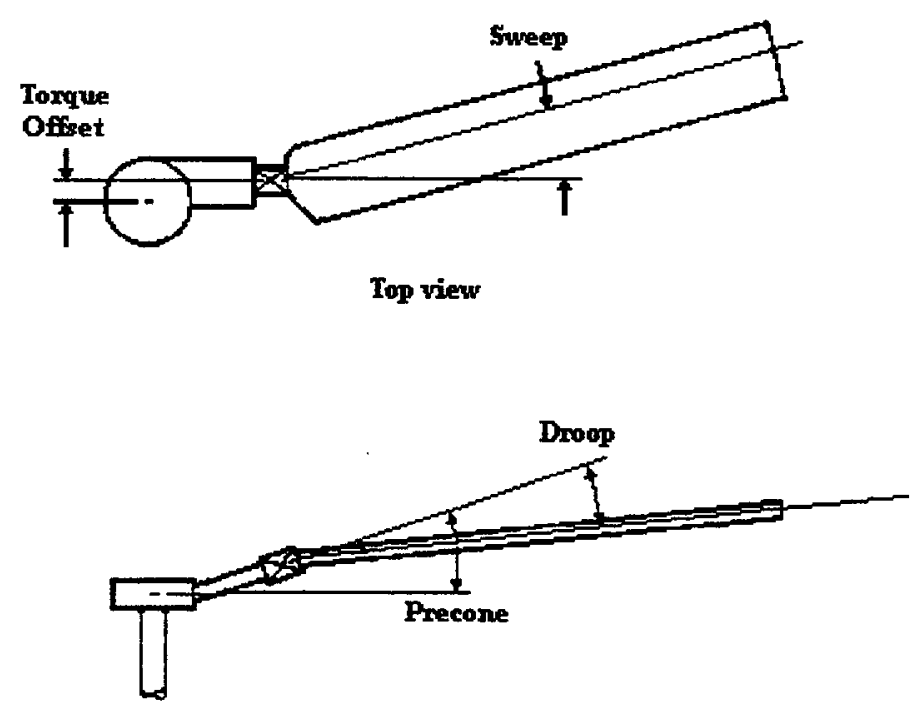

Side view

Figure 3.1: Possible hingeless rotor blade configurations

\section{Ordering Scheme}

Like the ordering scheme described in Chapter 2 for the cross-sectional analysis, a similar ordering scheme was used by Hodges and Ormiston to control higher order terms. To distinguish between the order $\varepsilon$ used by Badir, the variable $\underline{\varepsilon}$ will apply to the ordering scheme of Hodges and Ormiston. However, this ordering system is based differently on the magnitudes of nondimensional deflections in the axial, lead-lag, and flap directions defined by Hodges and Ormiston. The order $\underline{\varepsilon}$ refers to those magnitudes of deflection in lead-lag and flap whereas axial deflections are taken to be one order of magnitude smaller, $O\left(\underline{\varepsilon}^{2}\right)$. Within the energy expressions given in Hamilton's principle terms greater than $O\left(\underline{\varepsilon}^{2}\right)$ are ignored with respect to unity. The major ordering guidelines to which the energy expressions are based upon are presented in Appendix A.2. Although the ordering scheme has been carried out for this modified formulation for an active blade, no further details will be discussed in this thesis; Hodges and Ormiston also present a complete analysis. 


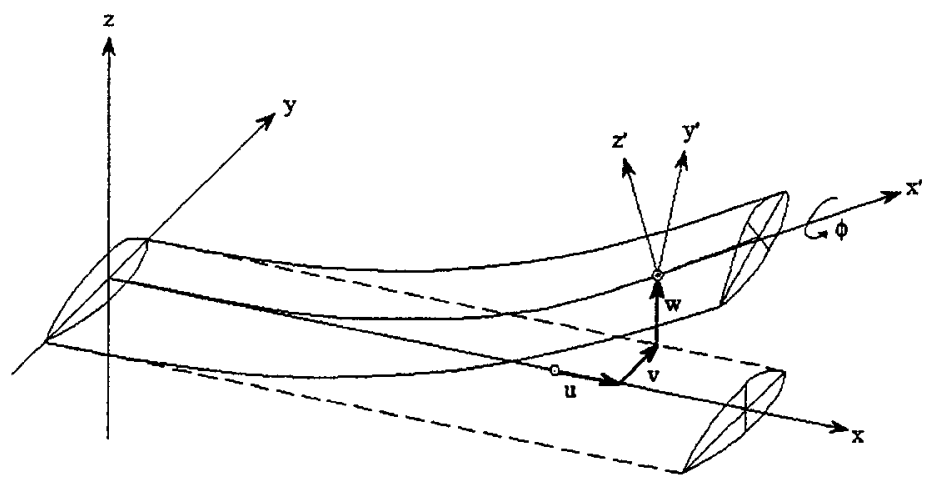

Figure 3.2: Elastic displacements

\subsection{Hamilton's Principle}

The dynamic equations of motion are obtaining by uniting the strain and inertial energies of the system as well as the work done by the external forces, namely aerodynamic loads.

Hamilton's principle states that as a particle moves from time $t_{1}$ to $t_{2}$ one has,

$$
\int_{t_{1}}^{t_{2}}[\delta(U-T)-\delta W] d t=0
$$

where $\delta U$ and $\delta T$ are the variations of strain and kinetic energies and $\delta W$ is the virtual work of external loads.

\subsubsection{Reference Frame}

Consider the reference frame of Figure 3.2 deformed to a state where deflections are given along the $x, y$, and $z$ axes and the blade twists through an angle $\phi$. Also, deformation in the axial direction is considered to be much smaller than in the other two directions. Figure 3.2 illustrates the deformed position of the blade with $u, v$, and $w$ denoting displacements relative to the elastic axis, $x$, and also $y$ and $z$ axes.

Between the deformations described by Badir and those defined by Hodges and Ormis- 


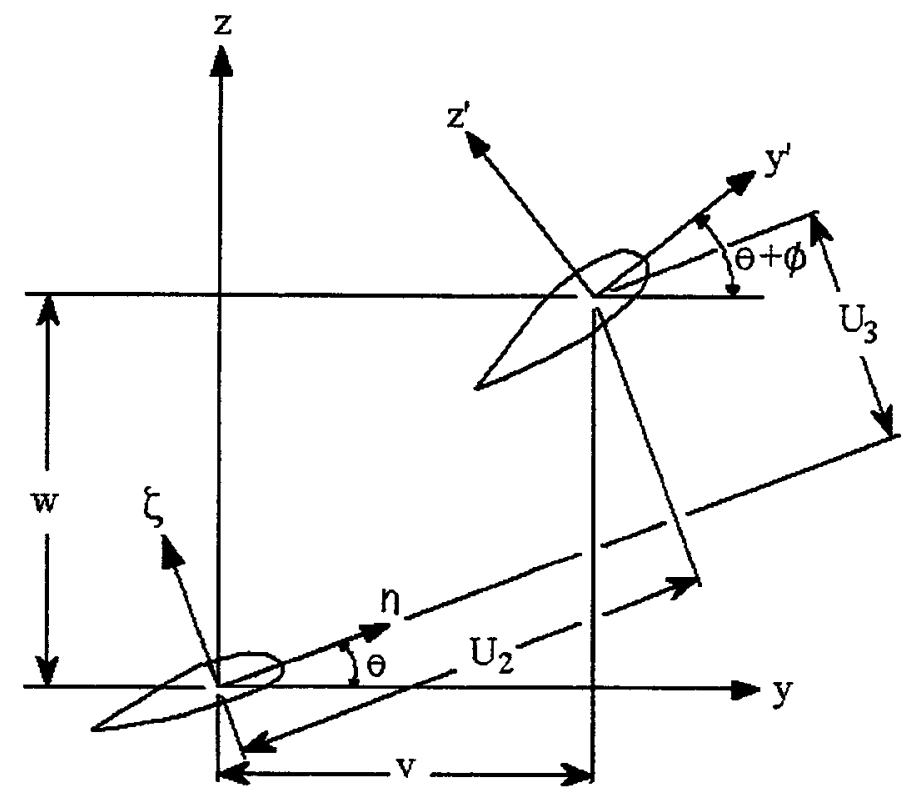

Figure 3.3: Undeformed and deformed blade cross-sections between reference frames

ton, relationships can be formed,

$$
\begin{gathered}
U_{1}=u \\
\varphi=\phi \\
U_{2}=v \cos \theta+w \sin \theta \\
U_{3}=-v \sin \theta+w \cos \theta
\end{gathered}
$$

which may also be illustrated in the $y$ and $z$ axis frame as shown by Figure 3.3. The pretwist of the blade, $\theta$, is also shown. While these relationships are not needed to relate the moments in bending and torsion due to piezoelectric actuation originating in the 2D linear cross-sectional analysis, with the beam formulation, they do provide a basis for comparison. Variables $\eta$ and $\zeta$ represent the blade cross-section principal axes coordinates. 


\subsubsection{Strain Energy}

To incorporate both structural and actuation terms from the cross-sectional analysis, first consider the strain energy, $U$, of a long, slender, homogeneous, and isotropic beam. In terms of engineering stresses and strains, one has,

$$
U=\frac{1}{2} \int_{0}^{L} \iint_{A}\left(\sigma_{x x} \varepsilon_{x x}+\sigma_{x \eta} \varepsilon_{x \eta}+\sigma_{x \zeta} \varepsilon_{x \zeta}\right) d \eta d \zeta d x
$$

where stresses are related to strains by Young's modulus, $E$, and Shear Modulus, $G$, by

$$
\begin{aligned}
& \sigma_{x x}=E \varepsilon_{x x} \\
& \sigma_{x \eta}=G \varepsilon_{x \eta} \\
& \sigma_{x \zeta}=G \varepsilon_{x \zeta}
\end{aligned}
$$

The variation of this strain energy is given by

$$
\begin{aligned}
\delta U= & \int_{0}^{L}\left\{V_{x^{\prime}}\left(\delta u^{\prime}+v^{\prime} \delta v^{\prime}+w^{\prime} \delta w^{\prime}\right)+\left(S_{x^{\prime}}+T_{x^{\prime}}\right) \delta \phi^{\prime}+P_{x^{\prime}} \delta \phi^{\prime \prime}\right. \\
& +\left[M_{z^{\prime}} \cos (\theta+\phi)+M_{y^{\prime}} \sin (\theta+\phi)\right]\left(\delta v^{\prime \prime}+w^{\prime \prime} \delta \phi\right) \\
& \left.+\left[M_{z^{\prime}} \sin (\theta+\phi)-M_{y^{\prime}} \cos (\theta+\phi)\right]\left(\delta w^{\prime \prime}-v^{\prime \prime} \delta \phi\right)\right\} d x
\end{aligned}
$$

and is obtained through the variation of strains $\varepsilon_{x x}, \varepsilon_{x \eta}$, and $\varepsilon_{x \zeta}$, whose expressions are

$$
\begin{aligned}
\delta \varepsilon_{x x}= & \delta u^{\prime}+v^{\prime} \delta v^{\prime}+w^{\prime} \delta w^{\prime}+\left(\eta^{2}+\zeta^{2}\right)(\theta+\phi)^{\prime} \delta \phi^{\prime}-\lambda \delta \phi^{\prime \prime} \\
& -[\eta \cos (\theta+\phi)-\zeta \sin (\theta+\phi)]\left(\delta v^{\prime \prime}+w^{\prime \prime} \delta \phi\right) \\
& -[\eta \sin (\theta+\phi)+\zeta \cos (\theta+\phi)]\left(\delta w^{\prime \prime}-v^{\prime \prime} \delta \phi\right) \\
\delta \varepsilon_{x \eta}= & -\hat{\zeta} \delta \phi^{\prime} \\
\delta \varepsilon_{x \zeta}= & -\hat{\eta} \delta \phi^{\prime}
\end{aligned}
$$


In Eq. (3.3), $V_{x^{\prime}}$ is the axial stress resultant along the $x^{\prime}$-axis. $M_{y^{\prime}}$ and $M_{z^{\prime}}$ are the bending moments about the lead-lag and flap axes respectively. Moreover, the twisting moment, $M_{x^{\prime}}$ with respect to the $x^{\prime}$-axis can be represented as:

$$
M_{x^{\prime}}=T_{x^{\prime}}+S_{x^{\prime}}-\left(P_{x^{\prime}}\right)^{\prime}
$$

where $T_{x^{\prime}}$ represents the twisting moment that arises from the moment of the component of force due to longitudinal stress that is normal to the elastic axis; $S_{x^{\prime}}$ is the twisting moment due to shear; and $P_{x^{\prime}}$ represents the work done by the longitudinal stress acting through the virtual displacement in the longitudinal direction due to warp. Warping is defined through the variable, $\lambda$, with the sectional coordinates, $\eta$ and $\zeta$ related to warping by $\hat{\eta}=\eta-\partial \lambda / \partial \zeta$ and $\hat{\zeta}=\zeta-\partial \lambda / \partial \eta$. Explicitly, one has:

$$
\begin{aligned}
V_{x^{\prime}} \equiv & \iint_{A} \sigma_{x x} d \eta d \zeta=E A\left\{u^{\prime}+\frac{v^{2}}{2}+\frac{w^{\prime 2}}{2}+k_{A}^{2} \theta^{\prime} \phi^{\prime}\right\} \\
& -E A\left\{e_{A}\left[v^{\prime \prime} \cos (\theta+\phi)+w^{\prime \prime} \sin (\theta+\phi)\right]\right\} \\
T_{x^{\prime}} \equiv & \iint_{A}(\theta+\phi)^{\prime} \sigma_{x x}\left(\eta^{2}+\zeta^{2}\right) d \eta d \zeta=E A k_{A}^{2}(\theta+\phi)^{\prime}\left(u^{\prime}+\frac{v^{\prime 2}}{2}+\frac{w^{2}}{2}\right) \\
& +E B_{1}^{\star} \theta^{\prime 2} \phi^{\prime}-E B_{2}^{\star} \theta^{\prime}\left(v^{\prime \prime} \cos \theta+w^{\prime \prime} \sin \theta\right) \\
P_{x^{\prime}} \equiv & -\iint_{A} \lambda \sigma_{x x} d \eta d \zeta=E C_{1} \phi^{\prime \prime}+E C_{1}^{\star}\left(w^{\prime \prime} \cos \theta-v^{\prime \prime} \sin \theta\right) \\
S_{x^{\prime}} \equiv & \iint_{A}\left(\hat{\eta} \sigma_{x \zeta}-\hat{\zeta} \sigma_{x \eta}\right) d \eta d \zeta=G J \phi^{\prime} \\
M_{y^{\prime}} \equiv & \iint_{A} \zeta \sigma_{x x} d \eta d \zeta=E I_{y^{\prime}}\left[v^{\prime \prime} \sin (\theta+\phi)-w^{\prime \prime} \cos (\theta+\phi)\right]-E C_{1}^{\star} \phi^{\prime \prime} \\
M_{z^{\prime}} \equiv & -\iint_{A} \eta \sigma_{x x} d \eta d \zeta=E I_{z^{\prime}}\left[v^{\prime \prime} \cos (\theta+\phi)+w^{\prime \prime} \sin (\theta+\phi)\right] \\
& -E A e_{A}\left(u^{\prime}+\frac{v^{\prime 2}}{2}+\frac{v^{\prime 2}}{2}\right)-E B_{2}^{\star} \theta^{\prime} \phi^{\prime}
\end{aligned}
$$

where the cross-sectional properties for $A, I_{y}$, etc are given in Appendix $\mathrm{D}$ and defined below: 
$e_{A}:$ tension axis offset from the elastic axis

$A k_{A}^{2}$ : polar moment of inertia

$I_{y^{\prime}}, I_{z^{\prime}}$ : flapwise and chordwise moments of inertia

$J: \quad$ torsional constant including cross-section warping

(without warping $J$ is equal to polar moment of inertia)

$C_{1}$ : warping rigidity

$B_{1}, B_{1}^{\star}, C_{1}^{\star}$ : cross-sectional constants

The expressions of Eq. (3.5) need modification in the proper manner to take into account various issues, including anisotropic actuation, of the present work. There are four major factors to be considered.

First, it is recognized that, in general, two types of instability may be expected in hingeless rotor blades. The first is a structural instability arising from rotor blades with nonuniform stiffness distributions. This type of instability is a product of coupling between flap and lead-lag bending of the rotor blade. The structural coupling parameter, $\Re$, introduced earlier simulates variations in these couplings by relating the inboard and outboard segments of hingeless rotor blades. This coupling is a direct result of the degree of blade twist, $\theta$, of the outboard segment, while the inboard segment does not rotate. The parameter $\Re \theta$ is substituted for $\theta$ in the strain energy terms. This assumes that the average twist of a nonuniform blade is a fraction of the twist of a uniform blade. The current work assumes full flap-lag structural coupling, i.e. $\Re=1$. The second type of instability that is possible is primarily due to structural coupling between bending and torsion deflections of cantilever blades. This type of instability can normally be identified by coupled flap bending-torsion deflections as well as lead-lag bending of the rotor blade with a frequency close to the natural frequency of lead-lag bending. Assuming no chordwise offsets between the elastic, mass, tension, and aerodynamic centre axes will alleviate the primary source of concern for aeroelastic instability. Therefore the variables $e_{A}$ and 
$e$, referring to the offsets of the tension axes and mass centroid from the elastic axes, are assumed to be zero.

Secondly, based on the assumption of a homogeneous and isotropic material, the coefficients $E A, E I_{y^{\prime}}, E I_{z^{\prime}}$, and $G J$ in Eq. (3.5) represent the classical axial, bending, and torsion stiffness of a beam. In the case of a composite anisotropic rotor blade, ignoring coupling effects due to composite construction, at least as a first approximation, the diagonal terms $K_{11}, K_{22}, K_{33}$, and $K_{44}$ from constitutive Eq. (2.26) can be used to represent $E A, G J, E I_{y}$, and $E I_{z}$ respectively.

The third issue that will need addressing is the actuation force, $F_{1}^{(a)}$, and moments, $M_{1}^{(a)}, M_{2}^{(a)}$, and $M_{3}^{(a)}$ that were derived in Chapter 2. These terms will need to be incorporated into the corresponding expressions for stress resultants and moments, $V_{x^{\prime}}, S_{x^{\prime}}$, $M_{y^{\prime}}$, and $M_{z^{\prime}}$.

Finally, the equations of motion that will be developed for this torsionally flexible rotor blade will contain nonlinear inertial and structural terms which describe the nonlinear blade behaviour. The importance of these terms is recognized by retaining them throughout the derivations until the stability analyses. At this point the perturbation equations will be linearized, with coefficients representing any nonlinearities.

Not without importance, but also in limiting the scope of this work, a basic blade configuration without pretwist, i.e. $\theta^{\prime}=0$ is considered for the present work. As a result, Eq. (3.5) will now read:

$$
\begin{aligned}
V_{x^{\prime}} & \equiv \iint_{A} \sigma_{x x} d \eta d \zeta=E A\left\{u^{\prime}+\frac{v^{\prime 2}}{2}+\frac{w^{\prime 2}}{2}\right\}-F_{1^{\prime}}^{(a)} \\
T_{x^{\prime}} & \equiv \iint_{A}(\theta+\phi)^{\prime} \sigma_{x x}\left(\eta^{2}+\zeta^{2}\right) d \eta d \zeta=E A k_{A}^{2}(\theta+\phi)^{\prime}\left(u^{\prime}+\frac{v^{\prime 2}}{2}+\frac{w^{2}}{2}\right) \\
P_{x^{\prime}} & \equiv-\iint_{A} \lambda \sigma_{x x} d \eta d \zeta=E C_{1} \phi^{\prime \prime}+E C_{1}^{\star}\left(w^{\prime \prime} \cos \theta-v^{\prime \prime} \sin \theta\right) \\
S_{x^{\prime}} & \equiv \iint_{A}\left(\hat{\eta} \sigma_{x \zeta}-\hat{\zeta} \sigma_{x \eta}\right) d \eta d \zeta=G J \phi^{\prime}-M_{1^{\prime}}^{(a)}
\end{aligned}
$$




$$
\begin{aligned}
M_{y^{\prime}} & \equiv \iint_{A} \zeta \sigma_{x x} d \eta d \zeta=E I_{y^{\prime}}\left[v^{\prime \prime} \sin (\theta+\phi)-w^{\prime \prime} \cos (\theta+\phi)\right]-M_{2^{\prime}}^{(a)} \\
M_{z^{\prime}} & \equiv-\iint_{A} \eta \sigma_{x x} d \eta d \zeta=E I_{z^{\prime}}\left[v^{\prime \prime} \cos (\theta+\phi)+w^{\prime \prime} \sin (\theta+\phi)\right]-M_{3^{\prime}}^{(a)}
\end{aligned}
$$

However, in Eq. (3.6) the force and moment equations are with respect to the deformed frame of reference $x^{\prime}, y^{\prime}, z^{\prime}$, whereas the actuation force and moments given in Eqs. (2.26) and (2.27) are in the undeformed frame of reference $x, y, z$. Hence a coordinate transformation is required to express the actuation terms with respect to the deformed $x^{\prime}, y^{\prime}, z^{\prime}$ frame of reference.

Let $F_{1^{\prime}}^{(a)}, M_{1^{\prime}}^{(a)}, M_{2^{\prime}}^{(a)}$, and $M_{3^{\prime}}^{(a)}$ represent the actuation vector components in the deformed frame. This results in

$$
\begin{aligned}
F_{1^{\prime}}^{(a)} \equiv & F_{x^{\prime}}^{(a)}=F_{x}^{(a)} \\
M_{1^{\prime}}^{(a)} \equiv & M_{x^{\prime}}^{(a)}=M_{x}^{(a)}+v^{\prime} M_{y}^{(a)}+w^{\prime} M_{z}^{(a)} \\
M_{2^{\prime}}^{(a)} \equiv & M_{y^{\prime}}^{(a)}=-M_{x}^{(a)}\left[v^{\prime} \cos (\theta+\phi)+w^{\prime} \sin (\theta+\phi)\right]+M_{y}^{(a)} \cos (\theta+\phi) \\
& +M_{z}^{(a)} \sin (\theta+\phi) \\
M_{3^{\prime}}^{(a)} \equiv & M_{z^{\prime}}^{(a)}=M_{x}^{(a)}\left[v^{\prime} \sin (\theta+\phi)-w^{\prime} \cos (\theta+\phi)\right]-M_{y}^{(a)} \sin (\theta+\phi) \\
& +M_{z}^{(a)} \cos (\theta+\phi)
\end{aligned}
$$

where the details of this transformation are given in Appendix E.

At this point, integration by parts of the strain energy variation may be carried out to yield

$$
\delta U=\int_{0}^{L}\left(\bar{Y}_{u} \delta u+\bar{Y}_{v} \delta v+\bar{Y}_{w} \delta w+\bar{Y}_{\phi} \delta \phi\right) d x+b(U)
$$

where $b(U)$ contains the terms of the boundary conditions. A detailed discussion of boundary terms may be found in Hodges and Dowell. The expressions for $\bar{Y}_{u}, \bar{Y}_{v}, \bar{Y}_{w}$, and $\bar{Y}_{\phi}$ are 
given as

$$
\begin{aligned}
\bar{Y}_{u}= & -\left(V_{x^{\prime}}\right)^{\prime} \\
\bar{Y}_{v}= & {\left[M_{z^{\prime}} \cos (\theta+\phi)+M_{y^{\prime}} \sin (\theta+\phi)\right]^{\prime \prime}-\left(V_{x^{\prime}} v^{\prime}\right)^{\prime} } \\
\bar{Y}_{w}= & {\left[M_{z^{\prime}} \sin (\theta+\phi)-M_{y^{\prime}} \cos (\theta+\phi)\right]^{\prime \prime}-\left(V_{x^{\prime}} w^{\prime}\right)^{\prime} } \\
\bar{Y}_{\phi}= & \left(P_{x^{\prime}}\right)^{\prime \prime}-\left(S_{x^{\prime}}+T_{x^{\prime}}\right)^{\prime}-v^{\prime \prime}\left[M_{z^{\prime}} \sin (\theta+\phi)-M_{y^{\prime}} \cos (\theta+\phi)\right] \\
& +w^{\prime \prime}\left[M_{z^{\prime}} \cos (\theta+\phi)+M_{y^{\prime}} \sin (\theta+\phi)\right]
\end{aligned}
$$

\subsubsection{Kinetic Energy}

Consider now the expression for kinetic energy

$$
T=\frac{1}{2} \int_{0}^{L} \iint_{A} \rho \vec{V} \cdot \vec{V} d \eta d \zeta d x
$$

where $\vec{V}$ defines the velocity vector of an arbitrary point in a blade characterized by density, $\rho$. As shown by Hodges and Ormiston, this variation of kinetic energy can be expressed as:

$$
\delta T=\int_{0}^{L}\left(\hat{Z}_{u} \delta u+\hat{Z}_{v} \delta v+\hat{Z}_{w} \delta w+\hat{Z}_{\phi} \delta \phi\right) d x+b(T)
$$

where $b(T)$ contains the terms of the boundary conditions. The corresponding variables for each axis of deflection are

$$
\begin{aligned}
& \hat{Z}_{u}=m\left(\Omega^{2} x+2 \Omega \dot{v}\right) \\
& \hat{Z}_{v}=m \Omega^{2} v+2 m \Omega\left(\beta_{p c} \dot{w}-\dot{u}\right)-m \ddot{v} \\
& \hat{Z}_{w}=-m \beta_{p c}\left(\Omega^{2} x+2 \Omega \dot{v}\right)-m \ddot{w} \\
& \hat{Z}_{\phi}=-m k_{m}^{2} \ddot{\phi}-m \Omega^{2}\left(k_{m_{2}}^{2}-k_{m_{1}}^{2}\right) \cos (\theta+\phi) \sin (\theta+\phi)
\end{aligned}
$$


where the sectional properties as defined by the kinetic energy terms are also outlined in Appendix D.

\subsubsection{Virtual Work - Aerodynamic Loading}

Next, the virtual work done by aerodynamic forces are considered as,

$$
\delta W=\int_{0}^{L}\left(L_{u} \delta u+L_{v} \delta v+L_{w} \delta w+M_{\phi} \delta \phi\right) d x
$$

where $L_{u}, L_{v}, L_{w}$, and $M_{\phi}$ are the distributed loads that act in the $x, y$, and $z$ directions as well as the twisting moment about the elastic axis respectively. Based on Greenberg's extension of Theodorsen's theory [50] for a 2D airfoil undergoing sinusoidal motion in incompressible flow, it can be shown that,

$$
\begin{aligned}
L_{u} \cong & 0 \\
L_{v} \cong & \frac{\rho_{\infty} a c}{2}\left\{v_{i}^{2}-\Omega^{2} x^{2} \frac{c_{d}}{a}-\Omega x v_{i}(\theta+\phi)\right. \\
& \left.-\left[2 \Omega x \frac{c_{d}}{a}+(\theta+\phi) v_{i}\right] \dot{v}+\left[2 v_{i}-\Omega x(\theta+\phi)\right] \dot{w}\right\} \\
L_{w} \cong & \frac{\rho_{\infty} a c}{2}\left\{-\Omega x v_{i}+\Omega^{2} x^{2}\left(\theta+\phi+\int_{0}^{x} v^{\prime} w^{\prime \prime} d x\right)\right. \\
& -\Omega^{2} x v\left(\beta_{p c}+w^{\prime}\right)+\Omega^{2} \frac{x c}{2}\left(\beta_{p c}+w^{\prime}\right)+\left[2 \Omega x(\theta+\phi)-v_{i}\right] \dot{v} \\
& \left.-\Omega x \dot{w}+\frac{3 c}{4} \Omega x \dot{\phi}-\frac{c}{4} \ddot{w}\right\} \\
M_{\phi} \cong & -\frac{\rho_{\infty} a c}{2}\left(\frac{c^{2}}{8} \Omega x \dot{\phi}\right)
\end{aligned}
$$

where $\rho_{\infty}$ represents the density of air at sea level, $a$ is the lift chord slope, $c$ is the length of the chord, $\Omega$ is the helicopter rotor hover speed, $c_{d}$ is the coefficient of drag of the airfoil, $\beta_{p c}$ is the precone angle, and $v_{i}$ represents the induced inflow velocity. Furthermore, the aerodynamic load in the axial direction, $L_{u}$, is considered to be of a negligible order of 
magnitude and hence is assumed zero.

The induced inflow velocity $v_{i}$ is assumed to be steady and uniform along the blade. Its value is taken to be equal to the value of nonuniform flow at $x=0.75 L$ as determined by blade element momentum theory. The blade angle at $x=0.75 \mathrm{~L}$ is taken to be equal to the sum of blade collective pitch angle plus the equilibrium elastic twist $\phi_{0}$, at $x=0.75 \mathrm{~L}$. The expression for induced inflow velocity is given by:

$$
v_{i}=\operatorname{sgn}\left[\theta+\phi_{0}(0.75 L)\right] \Omega L \frac{\pi \sigma}{8}\left(\sqrt{1+\frac{12}{\pi \sigma}\left|\theta+\phi_{0}(0.75 L)\right|}-1\right)
$$

where $\sigma=\frac{b c}{\pi L}$ is the blade solidity for $b$ blades.

\subsection{Differential Equations}

The equations resulting from the integration of the strain, kinetic and aerodynamic terms are obtained through substitution of Eqs. (3.8), (3.11) and (3.13) into (3.1). This results in the total variational equation in terms of $u, v, w$, and $\phi$. Then, it is noted that for arbitrary variations $\delta u, \delta v, \delta w$, and $\delta \phi$, the coefficients of the variations in Hamilton's integrals must vanish for all $x$ along the blade as well as at the boundaries $x=0$ and $x=L$. One therefore obtains four nonlinear partial differential equations for $u, v, w$, and $\phi$

$\delta u$ equation:

$$
-T^{\prime}-m\left(\Omega^{2} x+2 \Omega \dot{v}\right)=L_{u}
$$

$\delta v$ equation:

$$
\begin{gathered}
-\left(T v^{\prime}\right)^{\prime}+\{-\cos (\theta+\phi) A A-\sin (\theta+\phi) B B \\
+\left[E I_{z^{\prime}} \cos ^{2}(\theta+\phi)+E I_{y^{\prime}} \sin ^{2}(\theta+\phi)\right] v^{\prime \prime}
\end{gathered}
$$




$$
\begin{array}{r}
\left.+\left[E I_{z^{\prime}}-E I_{y^{\prime}}\right] \cos (\theta+\phi) \sin (\theta+\phi) w^{\prime \prime}\right\}^{\prime \prime} \\
+2 m \Omega \dot{u}+m \ddot{v}-m \Omega^{2} v-2 m \Omega \beta_{p c} \dot{w}
\end{array}
$$$$
=L_{v}
$$

$\delta w$ equation:

$$
\begin{array}{r}
-\left(T w^{\prime}\right)^{\prime}+\{-\sin (\theta+\phi) A A+\cos (\theta+\phi) B B \\
+\left[E I_{z^{\prime}} \sin ^{2}(\theta+\phi)+E I_{y^{\prime}} \cos ^{2}(\theta+\phi)\right] w^{\prime \prime} \\
\left.+\left[E I_{z^{\prime}}-E I_{y^{\prime}}\right] \cos (\theta+\phi) \sin (\theta+\phi) v^{\prime \prime}\right\}^{\prime \prime} \\
+2 m \Omega \beta_{p c} \dot{v}+m \ddot{w}+m \Omega^{2} \beta_{p c} x
\end{array}
$$

$\delta \phi$ equation:

$$
\begin{gathered}
-\left[G J \phi^{\prime}-\left(M_{x}^{(a)}+v^{\prime} M_{y}^{(a)}+w^{\prime} M_{z}^{(a)}\right)+E A k_{A}^{2}(\theta+\phi)^{\prime}\left(u^{\prime}+\frac{v^{\prime 2}}{2}+\frac{w^{\prime 2}}{2}\right)\right]^{\prime} \\
-v^{\prime \prime}\left\{\left(E I_{z^{\prime}}\left[v^{\prime \prime} \cos (\theta+\phi)+w^{\prime \prime} \sin (\theta+\phi)\right]-A A\right) \sin (\theta+\phi)\right. \\
\left.-\left(E I_{y^{\prime}}\left[v^{\prime \prime} \sin (\theta+\phi)-w^{\prime \prime} \cos (\theta+\phi)\right]-B B\right) \cos (\theta+\phi)\right\} \\
+w^{\prime \prime}\left\{\left(E I_{z^{\prime}}\left[v^{\prime \prime} \cos (\theta+\phi)+w^{\prime \prime} \sin (\theta+\phi)\right]-A A\right) \cos (\theta+\phi)\right. \\
\left.+\left(E I_{y^{\prime}}\left[v^{\prime \prime} \sin (\theta+\phi)-w^{\prime \prime} \cos (\theta+\phi)\right]-B B\right) \sin (\theta+\phi)\right\} \\
+m k_{m}^{2} \ddot{\phi}+m \Omega^{2} \phi\left(k_{m_{2}}^{2}-k_{m_{1}}^{2}\right) \cos 2 \theta+m \Omega^{2}\left(k_{m_{2}}^{2}-k_{m_{1}}^{2}\right) \cos \theta \sin \theta=M_{\phi}
\end{gathered}
$$

with

$$
\begin{aligned}
& A A=\left[M_{x}^{(a)}\left(v^{\prime} \sin (\theta+\phi)-w^{\prime} \cos (\theta+\phi)\right)-M_{y}^{(a)} \sin (\theta+\phi)+M_{z}^{(a)} \cos (\theta+\phi)\right] \\
& B B=\left[-M_{x}^{(a)}\left(v^{\prime} \cos (\theta+\phi)+w^{\prime} \sin (\theta+\phi)\right)+M_{y}^{(a)} \cos (\theta+\phi)+M_{z}^{(a)} \sin (\theta+\phi)\right]
\end{aligned}
$$


where the tension, $T$ is given by

$$
T \equiv V_{x^{\prime}}=E A\left(u^{\prime}+\frac{v^{\prime 2}}{2}+\frac{w^{2}}{2}\right)-F_{x}^{(a)}
$$

which is true for constant pretwist angle $\left(\theta^{\prime}=0\right)$ and zero offset of the area centroid from the elastic axis. The boundary conditions reduce to zero: $b(U)-b(T)=0$. Refer to Bailey, and Hodges and Dowell for a more detailed analysis of the boundary conditions.

These equations, while appearing quite lengthy, may be reduced to the much simpler forms shown below

$\delta u$ equation:

$$
-T^{\prime}-m\left(\Omega^{2} x+2 \Omega \dot{v}\right)=0
$$

$\delta v$ equation:

$$
\begin{gathered}
-\left(T v^{\prime}\right)^{\prime}+\left[E I_{z^{\prime}}-\left(E I_{z^{\prime}}-E I_{y^{\prime}}\right) \sin ^{2} \theta\right] v^{\prime \prime \prime \prime} \\
+\left(E I_{z^{\prime}}-E I_{y^{\prime}}\right)\left[\frac{\sin 2 \theta}{2} w^{\prime \prime \prime \prime}-\sin 2 \theta\left(\phi v^{\prime \prime}\right)^{\prime \prime}+\cos 2 \theta\left(\phi w^{\prime \prime}\right)^{\prime \prime}\right] \\
+2 m \Omega\left(\dot{u}-\beta_{p c} \dot{w}\right)+m(\ddot{v}-\Omega v)+\left(M_{x}^{(a)} w^{\prime}\right)^{\prime \prime}-\left(M_{z}^{(a)}\right)^{\prime \prime}=L_{v}
\end{gathered}
$$

$\delta w$ equation:

$$
\begin{gathered}
-\left(T w^{\prime}\right)^{\prime}+\left[E I_{y^{\prime}}+\left(E I_{z^{\prime}}-E I_{y^{\prime}}\right) \sin ^{2} \theta\right] w^{\prime \prime \prime \prime} \\
+\left(E I_{z^{\prime}}-E I_{y^{\prime}}\right)\left[\frac{\sin 2 \theta}{2} v^{\prime \prime \prime \prime}+\cos 2 \theta\left(\phi v^{\prime \prime}\right)^{\prime \prime}+\sin 2 \theta\left(\phi w^{\prime \prime}\right)^{\prime \prime}\right] \\
+2 m \Omega \beta_{p c} \dot{v}+m \ddot{w}+m \beta_{p c} \Omega^{2} x-\left(M_{x}^{(a)} v^{\prime}\right)^{\prime \prime}+\left(M_{y}^{(a)}\right)^{\prime \prime}=L_{w}
\end{gathered}
$$

$\delta \phi$ equation:

$$
\left(E I_{z^{\prime}}-E I_{y^{\prime}}\right)\left[\left(w^{\prime \prime 2}-v^{\prime 2}\right) \frac{\sin 2 \theta}{2}+v^{\prime \prime} w^{\prime \prime} \cos 2 \theta\right]-G J \phi^{\prime \prime}+\left(M_{x}^{(a)}\right)^{\prime}
$$




$$
\begin{gathered}
+\left(v^{\prime} M_{y}^{(a)}\right)^{\prime}+\left(w^{\prime} M_{z}^{(a)}\right)^{\prime}+M_{x}^{(a)}\left(v^{\prime} v^{\prime \prime}+w^{\prime} w^{\prime \prime}\right)-M_{y}^{(a)} v^{\prime \prime}-M_{z}^{(a)} w^{\prime \prime} \\
-k_{A}^{2}\left(T \phi^{\prime}\right)^{\prime}+m k_{m}^{2} \ddot{\phi}+m \Omega^{2}\left(k_{m_{2}}^{2}-k_{m_{1}}^{2}\right) \phi \cos 2 \theta+m \Omega^{2}\left(k_{m_{2}}^{2}-k_{m_{1}}^{2}\right) \frac{\sin 2 \theta}{2}=M_{\phi}
\end{gathered}
$$

As discussed earlier, important coupling terms are retained in these nonlinear integropartial differential equations of motion. For example, the lead-lag or $\delta v$ equation contains the Coriolis term $2 m \Omega \dot{u}$ and the axial equation, $\delta u$, equation contains the centrifugal force of the lead-lag deflection velocity, $-2 m \Omega \dot{v}$. Those terms originating from the strain energy equations and which are a function of $\theta$, also contain full flap-lag coupling ( $\Re \theta$ where $\Re=1$ ) as outlined in Section 3.1.2.

Further reduction of the differential equations is possible with the realization that the number of independent generalized coordinates is three, rather than four, with $L_{u}$ being neglected as a higher order term and $u$ expressed in terms of $v, w$, and $\phi$. In addition, the tension term $T$ is also eliminated when expressed in terms of the other three variables.

Integration of Eq. (3.21) results in the tension term,

$$
T=\frac{m \Omega^{2}}{2}\left(L^{2}-x^{2}\right)+\int_{x}^{L} 2 m \Omega \dot{v} d x
$$

Substituting Eq. (3.25) into Eq. (3.20) and rearranging for $u^{\prime}$ gives

$$
u^{\prime}=\frac{m \Omega^{2} L^{2}}{E A}\left(\frac{1-x^{2} / L^{2}}{2}\right)+\frac{2 m \Omega^{2} L^{2}}{E A} \int_{x}^{L} \frac{\dot{v}}{\Omega L^{2}} d x-\frac{v^{\prime 2}}{2}-\frac{w^{\prime 2}}{2}+\frac{F_{x}^{(a)}}{E A}
$$

From Eq. (3.26) one obtains,

$$
\dot{u}=\frac{2 m \Omega^{2} L^{2}}{E A} \int_{0}^{x} \int_{x}^{L} \frac{\ddot{v}}{\Omega L^{2}} d x-\frac{1}{2} \int_{0}^{x}\left(2 v^{\prime} \dot{v}^{\prime}+2 w^{\prime} \dot{w^{\prime}}\right) d x+\frac{\dot{F}_{x}{ }^{(a)}}{E A} x
$$

For sufficiently large values of the dimensionless radial stiffness, $E A / m \Omega^{2} L^{2}$, the first term of Eq. (3.27) may be assumed negligible. By stating this, it signifies that the rotor 
blade is inextensional in the $x$-direction for perturbation bending deflections. Design results in Chapter 4 indicate that this assumption is valid for the current application. The last term, which is a time derivative of the applied load, may be kept for variable applied loads from the active plies, but this applied current is not a function of the position on the blade, $x$.

Further reduction of Eqs. (3.22) to (3.24) by eliminating $u$ and $T$ results in $\delta v$ equation:

$$
\begin{aligned}
&-\frac{m \Omega^{2}}{2}\left[v^{\prime}\left(L^{2}-x^{2}\right)\right]^{\prime}-2 m \Omega\left(v^{\prime} \int_{x}^{L} \dot{v} d x\right)^{\prime}+\left[E I_{z^{\prime}}-\left(E I_{z^{\prime}}-E I_{y^{\prime}}\right) \sin ^{2} \theta\right] v^{\prime \prime \prime \prime} \\
&+\left(E I_{z^{\prime}}-E I_{y^{\prime}}\right) \frac{\sin (2 \theta)}{2} w^{\prime \prime \prime \prime}+\left(E I_{z^{\prime}}-E I_{y^{\prime}}\right)\left[-\sin (2 \theta)\left(\phi v^{\prime \prime}\right)^{\prime \prime}+\cos (2 \theta)\left(\phi w^{\prime \prime}\right)^{\prime \prime}\right] \\
&+\left(M_{x}^{(a)} w^{\prime}\right)^{\prime \prime}-\left(M_{z}^{(a)}\right)^{\prime \prime}-2 m \Omega \beta_{p c} \dot{w}-2 m \Omega \int_{0}^{x}\left(v^{\prime} \dot{v}^{\prime}+w^{\prime} \dot{w}^{\prime}\right) d x \\
&+2 m \Omega \frac{\dot{F}_{x}^{(a)}}{E A} x+m\left(\ddot{v}-\Omega^{2} v\right)=L_{v}
\end{aligned}
$$

$\delta w$ equation:

$$
\begin{aligned}
&- \frac{m \Omega^{2}}{2}\left[w^{\prime}\left(L^{2}-x^{2}\right)\right]^{\prime}-2 m \Omega\left(w^{\prime} \int_{x}^{L} \dot{v} d x\right)^{\prime}+\left[E I_{y^{\prime}}+\left(E I_{z^{\prime}}-E I_{y^{\prime}}\right) \sin ^{2} \theta\right] w^{\prime \prime \prime \prime} \\
&+\left(E I_{z^{\prime}}-E I_{y^{\prime}}\right) \frac{\sin (2 \theta)}{2} v^{\prime \prime \prime \prime \prime}+\left(E I_{z^{\prime}}-E I_{y^{\prime}}\right)\left[\cos (2 \theta)\left(\phi v^{\prime \prime}\right)^{\prime \prime}+\sin (2 \theta)\left(\phi w^{\prime \prime}\right)^{\prime \prime}\right] \\
&-\left(M_{x}^{(a)} v^{\prime}\right)^{\prime \prime}+\left(M_{y}^{(a)}\right)^{\prime \prime}+2 m \Omega \beta_{p c} \dot{v}+m \ddot{w}+m \beta_{p c} \Omega^{2} x=L_{w}
\end{aligned}
$$

$\delta \phi$ equation:

$$
\begin{gathered}
-k_{A}^{2}\left\{\left(\frac{m \Omega^{2}}{2}\left(L^{2}-x^{2}\right)\right) \phi^{\prime}\right\}^{\prime}-G J \phi^{\prime \prime}+\left(M_{x}^{(a)}\right)^{\prime}+\left(v^{\prime} M_{y}^{(a)}\right)^{\prime} \\
+\left(w^{\prime} M_{z}^{(a)}\right)^{\prime}+\left(E I_{z^{\prime}}-E I_{z^{\prime}}\right)\left[\left(w^{\prime \prime 2}-v^{\prime \prime 2}\right) \frac{\sin (2 \theta)}{2}+v^{\prime \prime} w^{\prime \prime} \cos (2 \theta)\right] \\
\quad+M_{x}^{(a)}\left(v^{\prime} v^{\prime \prime}+w^{\prime} w^{\prime \prime}\right)-M_{y}^{(a)} v^{\prime \prime}-M_{z}^{(a)} w^{\prime \prime}+m k_{m}^{2} \ddot{\phi} \\
+m \Omega^{2}\left(k_{m_{2}}^{2}-k_{m_{1}}^{2}\right) \phi \cos (2 \theta)+m \Omega^{2}\left(k_{m_{2}}^{2}-k_{m_{1}}^{2} \frac{\sin (2 \theta)}{2}=M_{\phi}\right.
\end{gathered}
$$




\subsection{Nondimensional Equations}

Before proceeding with Galerkin's method, it is desirable to convert the above integropartial differential equations into nondimensional equations. In order to nondimensionalise the lead-lag and flap equations, they are divided through by $m \Omega^{2} L$, whereas the torsion equations are divided through by $m \Omega^{2} L^{2}$. The results for an active nonuniform blade combining Eq. (3.14) with Eqs. (3.28) to (3.30) are as follows:

$\delta \bar{v}$ equation:

$$
\begin{aligned}
& -\left[\bar{v}^{\prime} \int_{\bar{x}}^{1}(\bar{x}+2 \dot{\bar{v}}) d \bar{x}\right]^{\prime}+\Lambda_{2} \bar{v}^{\prime \prime \prime \prime}-\left(\Lambda_{2}-\Lambda_{1}\right) \sin ^{2} \theta \bar{v}^{\prime \prime \prime \prime} \\
& +\left(\Lambda_{2}-\Lambda_{1}\right) \frac{\sin 2 \theta}{2} \bar{w}^{\prime \prime \prime \prime}+\left(\Lambda_{2}-\Lambda_{1}\right)\left[-\sin (2 \theta)\left(\phi \bar{v}^{\prime \prime}\right)^{\prime \prime}+\cos (2 \theta)\left(\phi \bar{w}^{\prime \prime}\right)^{\prime \prime}\right] \\
& -2 \beta_{p c} \dot{\bar{w}}-2 \int_{0}^{\bar{x}}\left(\bar{v}^{\prime} \dot{\bar{v}}^{\prime}+\bar{w}^{\prime} \dot{\bar{w}}^{\prime}\right) d \bar{x}+\ddot{\bar{v}}-\bar{v} \\
& +\frac{\gamma}{6}\left\{\bar{x} \bar{v}_{i} \phi+\left[2 \frac{c_{d}}{a} \bar{x}+(\theta+\phi) \bar{v}_{i}\right] \dot{\bar{v}}-\left[2 \bar{v}_{i}-\bar{x}(\theta+\phi)\right] \dot{\bar{w}}\right\}+2 \frac{\dot{\bar{F}}_{x}^{(a)}}{\Delta} \bar{x} \\
& +\left(\bar{M}_{x}^{(a)}\right)^{\prime \prime} \bar{w}^{\prime}+2\left(\bar{M}_{x}^{(a)}\right)^{\prime} \bar{w}^{\prime \prime}+\bar{M}_{x}^{(a)} \bar{w}^{\prime \prime \prime}-\left(\bar{M}_{z}^{(a)}\right)^{\prime \prime} \\
& =\frac{\gamma}{6}\left(\bar{v}_{i}{ }^{2}-\frac{c_{d}}{a} \bar{x}^{2}-\bar{x} \bar{v}_{i} \theta\right)
\end{aligned}
$$

$\delta \bar{w}$ equation:

$$
\begin{aligned}
& -\left[\bar{w}^{\prime} \int_{\bar{x}}^{1}(\bar{x}+2 \dot{\bar{v}}) d \bar{x}\right]^{\prime}+\Lambda_{1} \bar{w}^{\prime \prime \prime \prime}+\left(\Lambda_{2}-\Lambda_{1}\right) \sin ^{2} \theta \bar{w}^{\prime \prime \prime \prime} \\
& +\left(\Lambda_{2}-\Lambda_{1}\right) \frac{\sin 2 \theta}{2} \bar{v}^{\prime \prime \prime \prime}+\left(\Lambda_{2}-\Lambda_{1}\right)\left[\cos (2 \theta)\left(\phi \bar{v}^{\prime \prime}\right)^{\prime \prime}+\sin (2 \theta)\left(\phi \bar{w}^{\prime \prime}\right)^{\prime \prime}\right]+2 \beta_{p c} \dot{\bar{v}} \\
& +\ddot{\bar{w}}\left(1+\frac{\gamma \bar{c}}{24}\right)+\frac{\gamma}{6}\left\{-\bar{x}^{2}\left(\phi+\int_{0}^{\bar{x}} \bar{v}^{\prime} \bar{w}^{\prime \prime} d \bar{x}\right)+\bar{x} \bar{v}\left(\beta_{p c}+\bar{w}^{\prime}\right)-\frac{\bar{c}}{2} \bar{x} \bar{w}^{\prime}+\bar{x} \dot{\bar{w}}\right. \\
& \left.-[2 \bar{x}(\theta+\phi)-\bar{v}] \overline{\bar{v}}-\frac{3 \bar{c}}{4} \bar{x} \dot{\phi}\right\}-\left(\bar{M}_{x}^{(a)}\right)^{\prime \prime} \bar{v}^{\prime}-2\left(\bar{M}_{x}^{(a)}\right)^{\prime} \bar{v}^{\prime \prime}-\bar{M}_{x}^{(a)} \bar{v}^{\prime \prime \prime}+\left(\bar{M}_{y}^{(a)}\right)^{\prime \prime} \\
& =-\beta_{p c} \bar{x}+\frac{\gamma}{6}\left(-\bar{x} \bar{v}_{i}+\bar{x}^{2} \theta+\frac{\bar{c}}{2} \bar{x} \beta_{p c}\right)
\end{aligned}
$$


$\delta \phi$ equation:

$$
\begin{aligned}
& -\frac{\mu^{2} K}{2}\left[\left(1-\bar{x}^{2}\right) \phi^{\prime}\right]^{\prime}-\kappa \phi^{\prime \prime}+\frac{\gamma \bar{c}^{2}}{48} \bar{x} \dot{\phi}+\mu^{2} \ddot{\phi}+\left(\mu_{2}^{2}-\mu_{1}^{2}\right) \phi \cos (2 \theta) \\
& +\left(\Lambda_{2}-\Lambda_{1}\right)\left[\left(\frac{\bar{w}^{\prime \prime 2}-\bar{v}^{\prime 2}}{2}\right) \sin (2 \theta)+\bar{v}^{\prime \prime} \bar{w}^{\prime \prime} \cos (2 \theta)\right]+\left(\bar{M}_{x}{ }^{(a)}\right)^{\prime}+\bar{v}^{\prime \prime} \bar{M}_{y}{ }^{(a)} \\
& +\bar{v}^{\prime}\left(\bar{M}_{y}{ }^{(a)}\right)^{\prime}+\bar{w}^{\prime \prime} \bar{M}_{z}^{(a)}+\bar{w}^{\prime}\left(\bar{M}_{z}{ }^{(a)}\right)^{\prime}+\bar{M}_{x}^{(a)} \bar{v}^{\prime} \bar{v}^{\prime \prime}+\bar{M}_{x}{ }^{(a)} \bar{w}^{\prime} \bar{w}^{\prime \prime}-\bar{M}_{y}{ }^{(a)} \bar{v}^{\prime \prime}-\bar{M}_{z}{ }^{(a)} \bar{w}^{\prime \prime} \\
& =-\left(\mu_{2}^{2}-\mu_{1}^{2}\right) \frac{\sin (2 \theta)}{2}
\end{aligned}
$$

where the nondimensional displacements are given by $\bar{v}=v / L, \bar{w}=w / L$, and $\Delta=1 / E A$.

The dimensionless parameters of the above equations are described in further detail in Appendix F.1.

\subsection{Galerkin's Method}

There are several assumed solution methods that can be used to solve the eigenvalue problem for a nonlinear integro-partial differential equation, for example the Collocation method, Galerkin's method, or Rayleigh-Ritz. These solutions use approximations and are often iterative, in order to solve for the generalized coordinates ( $\bar{v}, \bar{w}$, and $\phi$ in this case). The method chosen here is Galerkin's method, which assumes the solutions in terms of a product series of modal generalized coordinates or coefficients and mode shape functions. Galerkin's method is an effective technique, in that convergence of a solution is achieved at $\mathrm{N}$ modes, and all boundary conditions must be satisfied. For stability analyses, Hodges and Ormiston state that "for practical hingeless rotor configurations, $\mathrm{N}=5$ gives suitably converged eigenvalues". At this point, there is no discernable change in the values of the eigenvalues for low frequency instabilities characteristic of hover flight - first flap, first lead-lag, and torsion frequencies.

The dimensionless bending and torsion deflections as expressed in the modal generalized 
coordinates and modal shape functions are

$$
\begin{aligned}
\bar{v} & =\sum_{j=1}^{N} V_{j}(\psi) \Psi_{j}(\bar{x}) \\
\bar{w} & =\sum_{j=1}^{N} W_{j}(\psi) \Psi_{j}(\bar{x}) \\
\phi & =\sum_{j=1}^{N} \Phi_{j}(\psi) \Theta_{j}(\bar{x})
\end{aligned}
$$

where $\psi=\Omega t$. The assumed mode shapes corresponding to the above equations are standard for a nonrotating uniform cantilever beam undergoing bending and torsion. They are,

$$
\begin{aligned}
\Psi_{j}(\bar{x}) & =\cosh \left(\beta_{j} \bar{x}\right)-\cos \left(\beta_{j} \bar{x}\right)-\alpha_{j}\left[\sinh \left(\beta_{j} \bar{x}\right)-\sin \left(\beta_{j} \bar{x}\right)\right] \\
\Theta_{j}(\bar{x}) & =\sqrt{2} \sin \left(\gamma_{j} \bar{x}\right)
\end{aligned}
$$

where $\gamma_{j}=\pi(j-1 / 2)$ and the constants $\alpha_{j}$ and $\beta_{j}$ are summarized in Chang and Craig [51].

The assumed solutions of Eq. (3.34) are substituted into the nondimensional expressions describing displacements in $v, w$, and $\phi$ in Eqs. (3.31) to (3.33). Application of Galerkin's integrals results in $3 \mathrm{~N}$ nonlinear, nonhomogeneous, constant coefficient ordinary differential equations in terms of modal generalized coordinates:

$\delta V_{i}$ equation:

$$
\begin{aligned}
& \sum_{j=1}^{N}\left\langle V_{j}\left\{D_{i j}+\left[\Lambda_{2}-\left(\Lambda_{2}-\Lambda_{1}\right) \sin ^{2} \theta\right] \beta_{j}^{4} \delta_{i j}-\delta_{i j}\right\}\right. \\
& +W_{j} \frac{\sin 2 \theta}{2}\left(\Lambda_{2}-\Lambda_{1}\right) \beta_{j}^{4} \delta_{i j}-E_{i j}^{(a)} \\
& +\left(\Lambda_{2}-\Lambda_{1}\right) \sum_{k=1}^{N} K_{j k i} \Phi_{j}\left[-V_{k} \sin (2 \theta)+W_{k} \cos (2 \theta)\right]
\end{aligned}
$$




$$
\begin{aligned}
& +\sum_{k=1}^{N} W_{k} B_{i j k}^{(a)}-2 \beta_{p c} \delta_{i j} \dot{W}_{j}+2 \sum_{k=1}^{N}\left(F_{i k j}-F_{j k i}\right) V_{k} \dot{V}_{j} \\
& -2 \sum_{k=1}^{N} F_{j k i} W_{k} \dot{W}_{j}+\delta_{i j} \ddot{V}_{j}+\frac{\gamma}{6}\left\{\bar{v}_{i} I_{i j} \Phi_{j}+\left(2 \frac{c_{d}}{a} E_{i j}\right.\right. \\
& \left.\left.\left.+\theta \bar{v}_{i} \delta_{i j}+\bar{v}_{i} \sum_{k=1}^{N} \Phi_{k} H_{i j k}\right) \dot{V}_{j}-\left(2 \bar{v}_{i} \delta_{i j}-\theta E_{i j}-\sum_{k=1}^{N} \Phi_{k} G_{i j k}\right) \dot{W}_{j}\right\}\right) \\
& =\frac{\gamma}{6}\left(\bar{v}_{i}^{2} A_{i}-\frac{c_{d}}{a} C_{i}-\bar{v}_{i} \theta B_{i}\right)
\end{aligned}
$$

$\delta W_{i}$ equation:

$$
\begin{aligned}
& \sum_{j=1}^{N}\left\langle V_{j} \frac{\sin (2 \theta)}{2}\left(\Lambda_{2}-\Lambda_{1}\right) \beta_{j}^{4} \delta_{i j}+W_{j}\left\{D_{i j}+\left[\Lambda_{1}\right.\right.\right. \\
& \left.\left.+\left(\Lambda_{2}-\Lambda_{1}\right) \sin ^{2} \theta\right] \beta_{j}^{4} \delta_{i j}\right\}+\left(\Lambda_{2}-\Lambda_{1}\right) \sum_{k=1}^{N} K_{j k i} \Phi_{j}\left[V_{k} \cos (2 \theta)\right. \\
& \left.+W_{k} \sin (2 \theta)\right]+F_{i j}^{(a)}-\sum_{k=1}^{N} V_{k} B_{i j k}^{(a)}+2 \beta_{p c} \delta_{i j} \dot{V}_{j} \\
& +2 \sum_{k=1}^{N} F_{i k j} W_{k} \dot{V}_{j}+\ddot{W}_{j}\left(1+\frac{\gamma \bar{c}}{24}\right) \delta_{i j}+\frac{\gamma}{6}\left\{-J_{i j} \Phi_{j}\right. \\
& +\sum_{k=1}^{N} L_{i j k} V_{j} W_{k}+\beta_{p c} E_{i j} V_{j}-\frac{\bar{c}}{2} O_{i j} W_{j}-\left(2 \theta E_{i j}+2 \sum_{k=1}^{N} \Phi_{k} G_{i j k}\right. \\
& \left.\left.\left.-\bar{v}_{i} \delta_{i j}\right) \dot{V}_{j}+E_{i j} \dot{W}_{j}-\frac{3 \bar{c}}{4} I_{i j} \dot{\Phi_{j}}\right\}\right\rangle \\
& =-\beta_{p c} B_{i}+\frac{\gamma}{6}\left(-\bar{v}_{i} B_{i}+\theta C_{i}+\frac{\bar{c}}{2} \beta_{p c} B_{i}\right)
\end{aligned}
$$

$\delta \Phi_{i}$ equation:

$$
\begin{aligned}
& \sum_{j=1}^{N}\left\langle\Phi_{j}\left\{\mu^{2} K N_{i j}+\left[\kappa \gamma_{j}^{2}+\left(\mu_{2}^{2}-\mu_{1}^{2}\right) \cos (2 \theta)\right] \delta_{i j}\right\}\right. \\
& +\left(\Lambda_{2}-\Lambda_{1}\right) \sum_{k=1}^{N} K_{i j k}\left[\frac{\sin (2 \theta)}{2}\left(W_{j} W_{k}-V_{j} V_{k}\right)+\cos (2 \theta) V_{j} W_{k}\right] \\
& +\mu^{2} \delta_{i j} \ddot{\Phi}_{j}+\frac{\gamma \bar{c}^{2}}{48} M_{i j} \dot{\Phi}_{j}-C_{i j}^{(a)}+\sum_{k=1}^{N}\left[-V_{k}\left(G_{i j k}^{(a)}+D_{i j k}^{(a)}\right)\right.
\end{aligned}
$$




$$
\begin{aligned}
& \left.\left.-W_{k}\left(I_{i j k}^{(a)}+H_{i j k}^{(a)}\right)+\sum_{l=1}^{N} J_{i j k l}^{(a)}\left(V_{k} V_{l}+W_{k} W_{l}\right)\right]\right\rangle \\
& =-\left(\mu_{2}^{2}-\mu_{1}^{2}\right) \frac{\sin (2 \theta)}{\sqrt{2} \gamma_{i}}
\end{aligned}
$$

where the Galerkin integral constants are given in Appendix F.

Equations (3.36) to (3.38) show the actuation terms denoted by a superscript ( $a$ ). These 'active' Galerkin integrals are obtained from the actuation force and moments represented by a series expansion of mode shapes,

$$
\begin{aligned}
& \bar{F}_{x}{ }^{(a)}=\sum_{j=1}^{N} \mathbf{F}_{x j}^{(a)}(\bar{x}) \\
& \bar{M}_{x}{ }^{(a)}=\sum_{j=1}^{N} \mathbf{M}_{x j}^{(a)}(\bar{x}) \\
& \bar{M}_{y}^{(a)}=\sum_{j=1}^{N} \mathrm{M}_{y j}^{(a)}(\bar{x}) \\
& \bar{M}_{z}{ }^{(a)}=\sum_{j=1}^{N} \mathrm{M}_{z j}^{(a)}(\bar{x})
\end{aligned}
$$

Bailey suggests that $\mathbf{F}_{x j}^{(a)}, \mathbf{M}_{x j}^{(a)}, \mathbf{M}_{y j}^{(a)}$, and $\mathbf{M}_{z j}^{(a)}$ represent the effect of actuation on each mode shape due to material properties and lay-up of the active plies. These may be called "activation mode shapes". The Galerkin integrals for these mode shapes are also found in Appendix F.

The equilibrium deflections and perturbation equations, as discussed above, can now be obtained from the $3 \mathrm{~N}$ nonlinear ordinary differential equations now defined for $V, W$, and $\Phi$. First let the displacements in lead-lag, flap, and torsion be composed of constant quantities which represent the steady-state displacements about the equilibrium, $V_{0 j}, W_{0 j}$ and $\Phi_{0 j}$, and unsteady time-dependent perturbations denoted by $\Delta V_{j}, \Delta W_{j}$ and $\Delta \Phi_{j}$ :

$$
V_{j}(\psi)=V_{0 j}+\Delta V_{j}(\psi)
$$




$$
\begin{aligned}
W_{j}(\psi) & =W_{0 j}+\Delta W_{j}(\psi) \\
\Phi_{j}(\psi) & =\Phi_{0 j}+\Delta \Phi_{j}(\psi)
\end{aligned}
$$

Substitution of the equilibrium quantities into Eqs. (3.36) to (3.38) results in $3 \mathrm{~N}$ nonlinear algebraic equations:

Lead-lag equation for $V_{0 j}$ :

$$
\begin{aligned}
& \sum_{j=1}^{N}\left\langle V_{0 j}\left\{D_{i j}+\left[\Lambda_{2}-\left(\Lambda_{2}-\Lambda_{1}\right) \sin ^{2} \theta\right] \beta_{j}^{4} \delta_{i j}-\delta_{i j}\right\}\right. \\
& +W_{0 j} \frac{\sin 2 \theta}{2}\left(\Lambda_{2}-\Lambda_{1}\right) \beta_{j}^{4} \delta_{i j} \\
& +\left(\Lambda_{2}-\Lambda_{1}\right) \sum_{k=1}^{N} K_{j k i} \Phi_{0 j}\left[-V_{0 k} \sin (2 \theta)+W_{0 k} \cos (2 \theta)\right] \\
& \left.+\sum_{k=1}^{N} W_{0 k} B_{i j k}^{(a)}-E_{i j}^{(a)}+\frac{\gamma}{6} \bar{v}_{i} I_{i j} \Phi_{0 j}\right\rangle \\
& =\frac{\gamma}{6}\left(\bar{v}_{i}^{2} A_{i}-\frac{c_{d}}{a} C_{i}-\bar{v}_{i} \theta B_{i}\right)
\end{aligned}
$$

Flap equation for $W_{0 j}$ :

$$
\begin{aligned}
& \sum_{j=1}^{N}\left\langle V_{0 j} \frac{\sin (2 \theta)}{2}\left(\Lambda_{2}-\Lambda_{1}\right) \beta_{j}^{4} \delta_{i j}+W_{0 j}\left\{D_{i j}+\left[\Lambda_{1}\right.\right.\right. \\
& \left.\left.+\left(\Lambda_{2}-\Lambda_{1}\right) \sin ^{2} \theta\right] \beta_{j}^{4} \delta_{i j}\right\}+\left(\Lambda_{2}-\Lambda_{1}\right) \sum_{k=1}^{N} K_{j k i} \Phi_{0 j}\left[V_{0 k} \cos (2 \theta)\right. \\
& \left.+W_{0 k} \sin (2 \theta)\right]+F_{i j}^{(a)}-\sum_{k=1}^{N} V_{0 k} B_{i j k}^{(a)} \\
& \left.+\frac{\gamma}{6}\left\{-J_{i j} \Phi_{0 j}+\sum_{k=1}^{N} L_{i j k} V_{0 j} W_{0 k}+\beta_{p c} E_{i j} V_{0 j}-\frac{\bar{c}}{2} O_{i j} W_{0 j}\right\}\right\rangle \\
& =-\beta_{p c} B_{i}+\frac{\gamma}{6}\left(-\bar{v}_{i} B_{i}+\theta C_{i}+\frac{\bar{c}}{2} \beta_{p c} B_{i}\right)
\end{aligned}
$$


Torsion equation for $\Phi_{0 j}$ :

$$
\begin{aligned}
& \sum_{j=1}^{N}\left\langle\Phi_{0 j}\left\{\mu^{2} K N_{i j}+\left[\kappa \gamma_{j}^{2}+\left(\mu_{2}^{2}-\mu_{1}^{2}\right) \cos (2 \theta)\right] \delta_{i j}\right\}\right. \\
& +\left(\Lambda_{2}-\Lambda_{1}\right) \sum_{k=1}^{N} K_{i j k}\left[\frac{\sin (2 \theta)}{2}\left(W_{0 j} W_{0 k}-V_{0 j} V_{0 k}\right)+\cos (2 \theta) V_{0 j} W_{0 k}\right] \\
& -C_{i j}^{(a)}+\sum_{k=1}^{N}\left[-V_{0 k}\left(G_{i j k}^{(a)}+D_{i j k}^{(a)}\right)\right. \\
& \left.\left.-W_{0 k}\left(I_{i j k}^{(a)}+H_{i j k}^{(a)}\right)+\sum_{l=1}^{N} J_{i j k l}^{(a)}\left(V_{0 k} V_{0 l}+W_{0 k} W_{0 l}\right)\right]\right\rangle \\
= & -\left(\mu_{2}^{2}-\mu_{1}^{2}\right) \frac{\sin (2 \theta)}{\sqrt{2} \gamma_{i}}
\end{aligned}
$$

that can be solved by, for example, the Newton-Raphson method to obtain the equilibrium states, $V_{0 j}, W_{0 j}$ and $\Phi_{0 j}$, also known as the trim or operating conditions. Next, substituting Eq. (3.40) into Eqs. (3.36) to (3.38), subtracting from the resulting equations the equilibrium quantities of Eqs. (3.41) to (3.43) and neglecting all nonlinear products, one obtains a set of $3 \mathrm{~N}$ linearized perturbation equations in the form

$$
[M]\{\ddot{X}\}+[C]\{\dot{X}\}+[K]\{X\}+\{F\}=0
$$

Appendix $\mathrm{G}$ provides the matrices for the mass $[M]$, damping $[C]$, and stiffness $[K]$ whereby the vector $\{X\}$ is

$$
\{X\}=\left\{\begin{array}{c}
\Delta V_{j} \\
\Delta W_{j} \\
\triangle \Phi_{j}
\end{array}\right\}
$$

with $j=1 \ldots \mathrm{N}$ and $\mathrm{N}=6$. The last term $\{F\}$ on the left hand side denotes the force due to actuation. The explicit expression for $\{F\}$ is found in Appendix G.

The next chapter describes the design process for the model blade. With an optimal design configuration, the stability and response of the blade can then be determined using 
the equations of motion developed in this chapter. The homogeneous dynamic equation of the active blade, Eq. (3.44) will provide the eigenvalues and eigenvectors that characterize the stability problem. 


\section{Chapter 4}

\section{Blade Modelling}

The next step in developing the new blade concept, which is to be called the active or smart blade, is to choose an existing helicopter blade, the inactive blade, to be used as a model for the study. Even though the new active blade concept will improve on the existing inactive blade design, certain design aspects of the inactive blade must be retained. This will ensure that the basic parameters of standard blade aerodynamics (e.g. Lock number), and structural and dynamic characteristics (e.g. natural frequencies) are kept. This chapter also focusses on the considerations of designing an active composite blade, which includes the embedded piezoelectrics.

The inactive blade, which was chosen for this study, is the one used on the Eurocopter BO105 light utility helicopter. The BO105 is a typical example of a four bladed hingeless helicopter whose only articulation comes from roller bearings for blade pitch change [52]. The blades are a graphite-epoxy composite AS4/3501-6 with a NACA 23012 cross-section airfoil shape. There is also an $8^{\circ}$ linear twist which has not been incorporated into the present design.

Along with maintaining the set aerodynamic and structural characteristics of the inactive blade, the active blade model is heavily dependent upon composite construction 
methods, as mentioned above. Some of the basic design considerations are:

1. orientation of plies

2. number of plies

3. number of active plies

4. position of web

5. sides of spar to be actuated

6. length of spar to be actuated

This chapter addresses these characteristics by first modelling the host or 'baseline' blade. More specifically, this baseline blade has no embedded piezoelectrics present. Beginning with the first item, two types of ply orientations have been defined by previous researchers as: circumferentially uniform stiffness with web (CUS/w) and circumferentially asymmetric stiffness with web (CAS/w) $[21,30,35,38,45,53]$. These orientations are tested on two different spar designs. The first is a box beam spar that is enclosed within a NACA airfoil. The second design is a more direct design in that its shape is a NACA airfoil section. In the process of narrowing down these candidates for a baseline blade model, the inactive properties of the list, such as the number of plies and the optimal web position, are evaluated.

The method by which the inactive blade is made into an active one is by a substitution of the graphite-epoxy plies with the AFC plies. Therefore the analytical results form the grounds for determining the number of active layers. This chapter presents the mathematical basis for modelling the actuation on a section of the blade. In this fashion, the 6 th item on the above list, the length of the blade to be actuated, is specified. 
Finally, before proceeding with the modelling process for the two-celled box beam and the two-celled spar designs, the computational code is validated against a two-celled thin-walled box beam that was presented by Cesnik and Shin [36].

\subsection{Ply Orientation}

It was determined both in theory and experimentally in initial stages of bonding piezoelectric actuators to cantilever beams, that $\mathrm{a} \pm 45^{\circ}$ actuator orientation angle on opposing surfaces induced the greatest twist - Chen and Chopra [24]. This accomplishment in trying to suppress beam vibrations was actually the result of a series of studies in bending and extension coupling behaviour in composites. In particular, the bending-shear and extensionshear couplings arising from directionally applied piezoelectrics were adding significance to the term, tailorability. The two distinct composite beam configurations discussed above (CUS and CAS) give rise to these couplings. The CUS configuration produces extensiontwist coupling with bending-transverse shear as an accompanying or parasitic coupling. The CAS configuration produces bending-twist coupling with extension-transverse shear parasitic coupling. These configurations are discussed in detail in subsections 4.1.1 and 4.1 .2 , respectively.

Investigations into these coupling behaviours to manipulate blade characteristics began with the application of extension, torsion and bending loads to arbitrary closed crosssections of laminated composite beams. Besides their various design advantages, such as low weight to strength and stiffness ratios, thin-walled composite beams have also been considered as lifting surfaces onto which actuators can be bonded or embedded for vibration control.

However, other methods also have been suggested to overcome the vibration problem. Loewy [1], led the investigation into vibratory hub loads which introduced a method called 
higher harmonic control (HHC). He showed that for an N-bladed rotor, the oscillatory N-1, $\mathrm{N}$, and $\mathrm{N}+1$ per revolution blade loads will evoke $\mathrm{N}$ per revolution forces to the helicopter fuselage. The swashplate was introduced as a $\mathrm{HHC}$ system so that when it was excited at these higher harmonics it would cancel the detrimental blade loads. However, due to several drawbacks of a swashplate such as high power requirements etc., a better solution was required. Individual blade control (IBC) was a natural progression into reducing the transfer of the heavy blade loads and which led investigators into studying tailored composite beams. In individual blade control each blade is mechanically altered, allowing for much larger band-widths than with the swashplate. This explains why tip twists on the order of $2^{\circ}$ at frequencies of $\mathrm{N} / \mathrm{rev}$ are needed.

Initial work into tailoring composite beams with piezoelectric actuators began with the work of Crawley and de Luis [18]. Experimental validation on a solid cross-section proved that shape control in extension and bending may be achieved with little effect on the passive structural properties through segmented surface mounted or embedded actuators. Later, Hodges recognized that work had begun quite rigourously into this topic and presented a review of methods achieved to-date [54]. The nonclassical bending-transverse shear couplings and torsional warping rigidity effects on a non-actuated thin-walled tubular beam were then addressed by Rehfield et al [45]. A circumferentially uniform stiffness (CUS) layup refers to the winding technique used in manufacturing this closed circular section. This group showed that without accounting for the presence of shear deformation, degrees of bending deflections will be grossly inaccurate - thus contradicting classical theory presented by [55]. Crawley and Lazarus began experimenting with these extension-twist and bending-twist couplings of composite plates with bonded PZT elements but achieved only small, albeit significant deflections, in [19]. Rehfield and Atilgan showed that coupling from the CUS or CAS layups also affects shear centre and elastic axis locations in composite thin-walled beams [21]. They showed that when shear forces are applied at the shear 
centre of a composite section there will be twisting. CUS and CAS were also referred to as antisymmetric and symmetric layups, respectively, by Chandra, Stemple and Chopra [43], who experimentally verified the couplings. Finally, a direct analytical formulation for predicting load deformation behaviour of tailored composite antisymmetric and symmetric box beams was developed by Smith and Chopra [44].

As coupling effects became better understood, research turned to bonding techniques and other actuation parameters. Barrett improved directional attachment techniques (Directionally Applied Piezoelectrics, DAP) and effects on a cantilever beam with isotropic PZT elements [22]. Chen and Chopra then experimented with the effects of bond thickness, actuator skew angle, and actuator spacing [24]. A CUS-style Froude-scale helicopter rotor blade was built with banks of embedded piezoelectric actuators at $\pm 45^{\circ}$ angles on the top and bottom surfaces. It was one of the first attempts to attain tip twists on a scaled helicopter blade model, but only $0.1^{\circ}$ of twist was achieved at $900 \mathrm{rpm}$, and again in [25], in a series of hover tests $0.5^{\circ}$ was achieved. With the introduction of the Piezoelectric Fibre Composite (PFC) by Bent [26], the potential applications and benefits of this new material were recognized. Bent, Hagood, and Rodgers experimenting on a PFC laminate, showed higher degrees of composite anisotropy than with DAP elements [29].

The current research utilises the PFC for blade modelling. The choice over whether to use a CUS or CAS layup is described in further detail below.

\subsubsection{Circumferentially Uniform Stiffness with Web, CUS/w}

Also known as an antisymmetric spar layup, the circumferentially uniform stiffness design was first applied to a beam by Rehfield et al [45]. An antisymmetric layup refers to manufacturing the cross-section by wrapping the composite using a winding technique. For example, for a box beam, ply layups on opposite sides of the box are reversed in orientation. This shall not be confused with classical notation for an anti-symmetric 


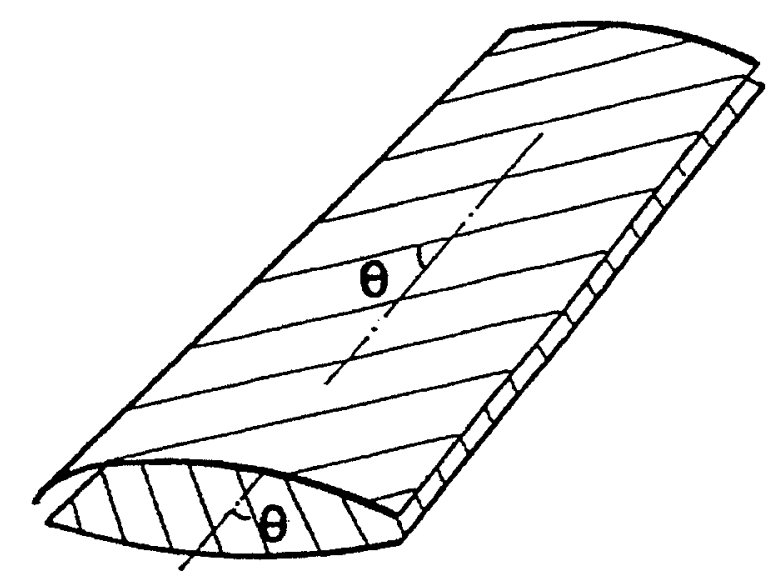

Figure 4.1: Circumferentially uniform stiffness configuration

laminate [48]. Figure 4.1 shows a CUS configuration [21]. From a bird's-eye-perspective $(\mathrm{BEP})$, which refers to a view from the top or also a view from the left or leading edge side, with $\theta$ measured with respect to the longitudinal or $x$-axis [53],

$$
\theta(y)_{t o p}=-\theta(y)_{b o t t o m}
$$

This type of configuration produces extension-twist coupling with bending-transverse shear parasitic coupling. Only if the cross-section is purely wound with equal material properties and number of layers between the top (t), right (r), bottom (b), and left (l) sides will the following relationships for stiffness hold true:

$$
\begin{aligned}
& A_{t}=A_{b}=A_{l}=A_{r} \\
& B_{t}=B_{b}=B_{l}=B_{r} \\
& C_{t}=C_{b}=C_{l}=C_{r}
\end{aligned}
$$

where $A, B$, and $C$ refer to the axial, coupling and in-plane shear stiffness. This results in a stiffness matrix with extension-twist couplings $\left(K_{12}\right)$, i.e. this matrix will now read as 
Table 4.1: Stiffness constants of CUS/w configuration

\begin{tabular}{|c|c|c|c|c|}
\hline View & Side & $\mathbf{A}$ & B & $\bar{C}$ \\
\hline $\begin{array}{c}\text { BEP } \\
\text { Unwound }\end{array}$ & $\begin{array}{c}\text { Top } \\
{[15 / 30 / 45]_{2}} \\
{[15 / 30 / 45]_{2}} \\
\end{array}$ & $4.935 E+07$ & $2.928 E+07$ & $4.481 E+07$ \\
\hline $\begin{array}{c}\text { BEP } \\
\text { Unwound } \\
\end{array}$ & $\begin{array}{c}\text { Bottom } \\
{[-45 /-30 /-15]_{2}} \\
{[15 / 30 / 45]_{2}} \\
\end{array}$ & $4.935 E+07$ & $2.928 E+07$ & $4.481 E+07$ \\
\hline $\begin{array}{c}\text { BEP } \\
\text { Unwound }\end{array}$ & $\begin{array}{c}\text { Left Vertical } \\
{[15 / 30 / 45]_{2}} \\
{[15 / 30 / 45]_{2}} \\
\end{array}$ & $4.935 E+07$ & $2.928 E+07$ & $4.481 E+07$ \\
\hline $\begin{array}{c}\text { BEP } \\
\text { Unwound }\end{array}$ & $\begin{array}{c}\text { Web } \\
{[-45 /-30 /-15] ;[15 / 30 / 45]} \\
{[15 / 30 / 45] ;[15 / 30 / 45]}\end{array}$ & $4.935 E+07$ & $2.928 E+07$ & $4.481 E+07$ \\
\hline $\begin{array}{c}\text { BEP } \\
\text { Unwound }\end{array}$ & $\begin{array}{c}\text { Right Vertical } \\
{[-45 /-30 /-15]_{2}} \\
{[15 / 30 / 45]_{2}} \\
\end{array}$ & $4.935 E+07$ & $2.928 E+07$ & $4.481 E+07$ \\
\hline
\end{tabular}

$$
\left[\begin{array}{cccc}
K_{11} & K_{12} & 0 & 0 \\
K_{12} & K_{22} & 0 & 0 \\
0 & 0 & K_{33} & 0 \\
0 & 0 & 0 & K_{44}
\end{array}\right]
$$

If the number of layers or material properties in a side change, these relationships are no longer valid. For multi-celled beams with a web (CUS/w), in order to maintain the relationships of Eq. (4.1) each half of the web must be of reverse orientation to its opposing side. This is best illustrated with an example. Table 4.1 gives stiffness values for a nominally 6 layered, two-celled box beam, where 'unwound' refers to observing the plies as if each cell were unwrapped. The two-celled box beam has equal height and cell widths of $25 \mathrm{~mm}$, and is modelled using the graphite-epoxy composite AS4/3501-6.

A side view of arbitrary top and bottom layers of a box beam in Figure 4.2, shows the BEP ply angle orientation needed to give the relationships of Eq. (4.1), much like an antisymmetric laminate configuration in classical laminate theory. Both a bird's-eye- 


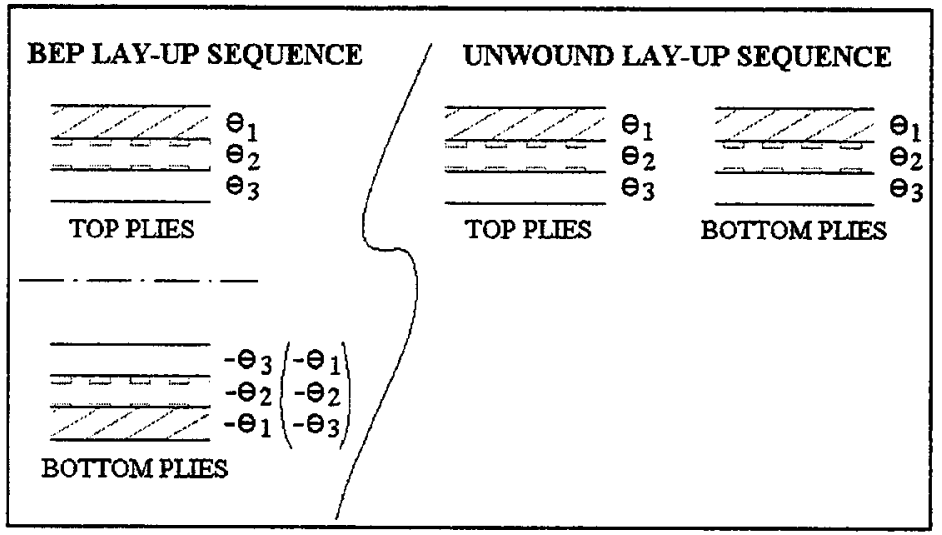

Figure 4.2: Lay-up sequences of top and bottom sides of CUS/w configuration

perspective and an unwound view are shown. Angles in brackets result in the same relationships as Eq. (4.1), but they do not represent a continuously wrapped configuration.

The primary design objective is to produce the most twist from a blade embedded with piezoelectric fibres. The CUS configuration achieves this objective by maximizing twist with $\pm 45^{\circ}$ plies oriented about the elastic axis and a large moment arm [30]. Therefore actuation capabilities for the laminates of the smart blade are reinforced by the passive blade coupling characteristics given by a CUS cross-section. As such, the CUS or antisymmetric layup is used in the final blade designs of this thesis.

\subsubsection{Circumferentially Asymmetric Stiffness with Web, CAS/w}

The circumferentially asymmetric stiffness configuration may be observed in Figure 4.3 [21]. Like CUS/w, CAS/w has been modified from a single cell case to a 2 cell case. The main difference between the two configurations is that in a CAS or symmetric cell layup opposite sides are mirror images. This is analogous to classical laminate theory for symmetric laminate layups in that every ply above the laminate midplane will have the same grouping below the midplane [48]. Bending-twist coupling with parasitic extensiontransverse shear coupling exists for this configuration. The following relationships apply 


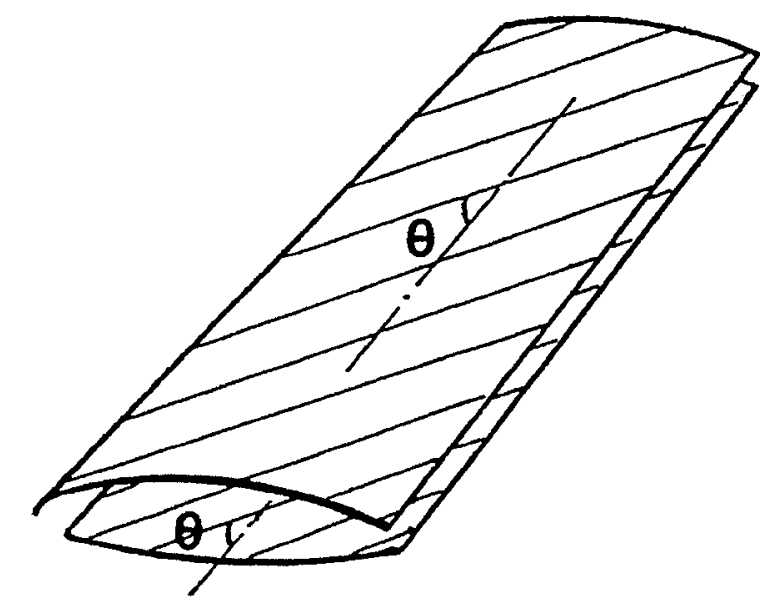

Figure 4.3: Circumferentially asymmetric stiffness configuration

for axial, coupling, and in-plane stiffness between sides:

$$
\begin{aligned}
& A_{t}=A_{b}=A_{l}=A_{r} \\
& B_{t}=-B_{b} ; \quad B_{l}=-B_{r} \\
& C_{t}=C_{b}=C_{l}=C_{r}
\end{aligned}
$$

resulting in a stiffness matrix containing bending-twist coupling terms $\left(K_{23}\right)$,

$$
\left[\begin{array}{cccc}
K_{11} & 0 & 0 & 0 \\
0 & K_{22} & K_{23} & 0 \\
0 & K_{23} & K_{33} & 0 \\
0 & 0 & 0 & K_{44}
\end{array}\right]
$$

where coupled stiffness values, $B$, are equal and opposite in sign for facing sides. For the web to have a value equal to that of one of the sides, it must have the same orientation and contain the same number of plies as one of the sides. Table 4.2 gives an example of a $\mathrm{CAS} / \mathrm{w}$ box beam.

Figure 4.4 shows the side views of a CAS configuration, with the bracketed orientations also giving equal stiffness values. This type of configuration is used when large bending 
Table 4.2: Stiffness constants of CAS/w configuration

\begin{tabular}{|c|c|c|c|c|}
\hline View & Side & $\overline{\mathbf{A}}$ & $\bar{B}$ & $\bar{C}$ \\
\hline $\begin{array}{c}\text { BEP } \\
\text { Unwound }\end{array}$ & $\begin{array}{c}\text { Top } \\
{[20 /-70 / 20 /-70 /-70 / 20]} \\
{[20 /-70 / 20 /-70 /-70 / 20]}\end{array}$ & $4.560 E+07$ & $2.871 E+07$ & $4.602 E+07$ \\
\hline $\begin{array}{c}\text { BEP } \\
\text { Unwound }\end{array}$ & $\begin{array}{c}\text { Bottom } \\
{[20 /-70 /-70 / 20 /-70 / 20]} \\
{[-20 / 70 /-20 / 70 / 70 /-20]}\end{array}$ & $4.560 E+07$ & $-2.871 E+07$ & $4.602 E+07$ \\
\hline $\begin{array}{c}\text { BEP } \\
\text { Unwound } \\
\end{array}$ & $\begin{array}{c}\text { Left Vertical } \\
{[20 /-70 / 20 /-70 /-70 / 20]} \\
{[20 /-70 / 20 /-70 /-70 / 20]}\end{array}$ & $4.560 E+07$ & $2.871 E+07$ & $4.602 E+07$ \\
\hline $\begin{array}{c}\text { BEP } \\
\text { Unwound }\end{array}$ & $\begin{array}{c}\text { Web } \\
{[20 /-70 / 20 /-70 /-70 / 20]} \\
{[20 /-70 / 20 /-70 /-70 / 20]}\end{array}$ & $4.560 E+07$ & $2.871 E+07$ & $4.602 E+07$ \\
\hline $\begin{array}{c}\text { BEP } \\
\text { Unwound }\end{array}$ & $\begin{array}{c}\text { Right Vertical } \\
{[20 /-70 /-70 / 20 /-70 / 20]} \\
{[-20 / 70 /-20 / 70 / 70 /-20]}\end{array}$ & $4.560 E+07$ & $-2.871 E+07$ & $4.602 E+07$ \\
\hline
\end{tabular}

moments are desired.

In addition, if any two adjacent plies of an arbitrary side have orientations equal and opposite in sign, it will result in null values of coupling stiffness, $B$. From classical laminate theory for a laminate symmetric with respect to the midplane, $B$ vanishes also; there is no coupling between extension and bending.

For either a CUS or CAS configuration the relationships between Eqs. (4.1) and (4.2),

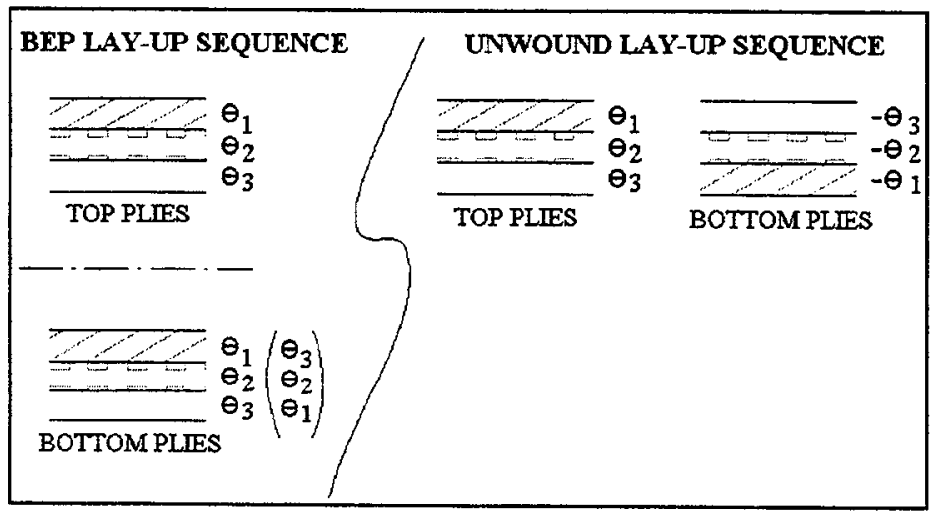

Figure 4.4: Lay-up sequences of top and bottom sides of CAS configuration 
as well as the stiffness matrices, will vary by changing either material properties, number of layers, or angle orientation between sides.

\subsection{Modal Actuation}

For an active fibre composite the shear force is transmitted throughout the length of the piezoelectric. As a result, the forces and moments produced by the actuating elements are summed over the length of the blade section containing piezoelectric components. Analytical results in Chapter 5 give a good indication of the percent of the blade requiring actuation authority. This section outlines the modal equations that apply to the actuation vector for $F_{x}^{(a)}, M_{x}^{(a)}, M_{y}^{(a)}$, and $M_{z}^{(a)}$.

The Galerkin integrals of Chapter 3 defined the deflections $v$ and $w$, and twist $\phi$ as a summation of an assumed solution composed of generalized coordinates and mode shape functions. The actuation force and moments were also given by a series expansion, as shown in Eq. (3.39). An active blade, which is a continuous system, is a function of both mode and position. These active shape functions may be broken down such that the influence of the $j$ th actuated force or moment term is a result of a ratio of the $j$ th mode shape over the sum of the mode shapes:

$$
\begin{aligned}
\mathbf{F}_{x j}^{(a)}(\bar{x}) & =\frac{\Theta_{j}^{\prime}(\bar{x})}{\sum_{k=1}^{N} \Theta_{k}^{\prime}(\bar{x})} \bar{F}_{x}^{(a)} \\
\mathbf{M}_{x j}^{(a)}(\bar{x}) & =\frac{\Theta_{j}^{\prime}(\bar{x})}{\sum_{k=1}^{N} \Theta_{k}^{\prime}(\bar{x})} \bar{M}_{x}^{(a)} \\
\mathbf{M}_{y j}^{(a)}(\bar{x}) & =\frac{\Psi_{j}^{\prime \prime}(\bar{x})}{\sum_{k=1}^{N} \Psi_{k}^{\prime \prime}(\bar{x})} \bar{M}_{y}^{(a)} \\
\mathbf{M}_{z j}^{(a)}(\bar{x}) & =\frac{\Psi_{j}^{\prime \prime}(\bar{x})}{\sum_{k=1}^{N} \Psi_{k}^{\prime \prime}(\bar{x})} \bar{M}_{z}^{(a)}
\end{aligned}
$$

The associated mode shape functions, $\Theta_{j}$ and $\Psi_{j}$, for each force and moment of the 
above equations are determined by the corresponding deflections given by the constitutive relationship of Eq. (2.26). Bending deflections $U_{2}$ and $U_{3}$ corresponding to actuation moments $M_{z}$ and $M_{y}$, respectively, require the use of the mode shape $\Psi_{j}$. Axial extension $F_{x}$ and torsion $M_{x}$ utilise the mode shape $\Theta_{j}$.

The Galerkin actuation integrals are given in Appendix F.2. These integrals may be divided into inactive and active portions along the longitudinal axis. For example, the active Galerkin integral $C_{i j}^{(a)}$ is written as

$$
C_{i j}^{(a)}=\int_{0}^{x_{1}} \Theta_{i}^{\prime} \mathbf{M}_{x j}^{(a)} d \bar{x}+\int_{x_{1}}^{x_{2}} \Theta_{i}^{\prime} \mathbf{M}_{x j}^{(a)} d \bar{x}+\int_{x_{2}}^{1} \Theta_{i}^{\prime} \mathbf{M}_{x j}^{(a)} d \bar{x}
$$

with the remaining integrals given in Appendix F.3. The positions $x_{1}$ and $x_{2}$ defined where the AFCs begin and end along the span. Integrating only over the length of the actuator, and substituting those mode shapes of Eq. (4.3) into Eq. (4.4) results in

$$
C_{i j}^{(a)}=\int_{x_{1}}^{x_{2}} \Theta_{i}^{\prime}(\bar{x}) \frac{\Theta_{j}^{\prime}(\bar{x})}{\sum_{k=1}^{N} \Theta_{k}^{\prime}(\bar{x})} \bar{M}_{x}^{(a)} d \bar{x}
$$

with the remaining integrals given in Appendix F.4.

This completes the process for analytically actuating the smart helicopter blade between points $x_{1}$ and $x_{2}$ along the blade span.

\subsection{Validation of Code}

Modelling for all blade designs was carried out using MATLAB version 6.5 software. In order to ensure that the code is computing correctly, it is necessary to validate it against published results. Cross-sectional stiffness constants, $K_{i j}$, produced by the author are compared with those values given by Bailey, and Cesnik and Shin. Appendices K and L contain these codes. 


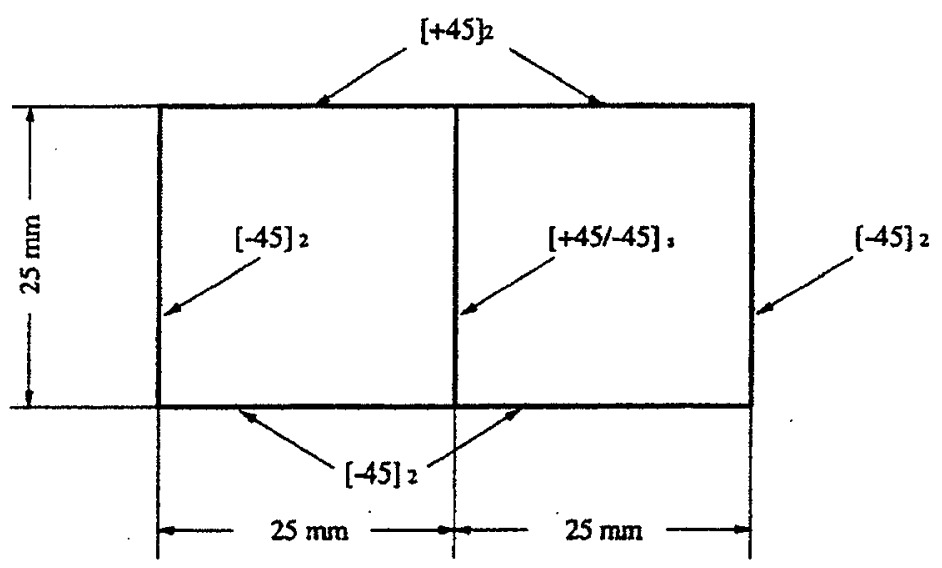

Figure 4.5: Two-celled thin-wall box beam used for validation

Table 4.3: Material properties of graphite-epoxy composite AS4/3501-6

\begin{tabular}{|c|c|}
\hline $\begin{array}{c}E_{L L}=142 \mathrm{GPa} \\
E_{N N}=E_{T T}=9.8 \mathrm{GPa} \\
G_{L T}=G_{L N}=6.0 \mathrm{GPa} \\
G_{T N}=4.80 \mathrm{GPa}\end{array}$ & $\begin{array}{c}\nu_{L T}=\nu_{L N}=0.3 \\
\nu_{T N}=0.42 \\
\text { thickness }=0.127 \mathrm{~mm} \\
\text { density }=1790 \mathrm{~kg} / \mathrm{m}^{3}\end{array}$ \\
\hline
\end{tabular}

The cross-sectional stiffness constants, $K_{i j}$, from the constitutive relationships for a two-celled beam were developed by Badir, but not verified. Cesnik and Shin validated these passive stiffness constants numerically against the asymptotically correct finite element based code, VABS (Variational-Asymptotical Beam Section Analysis) [56] - a model for arbitrary multi-celled geometries.

Figure 4.5 illustrates the two-celled box beam configuration without active plies used in the validation study. The material used for this application is also the graphite-epoxy composite AS4/3501-6. Material properties of this composite are given in Table 4.3. Maintaining the thin-walled restrictions given in Eq. (2.1), the ratio between thickness and maximum cross-sectional dimension must be much less than one.

Table 4.4 shows that the results obtained from the present formulation are in very good agreement with those obtained by Cesnik and Shin. There is also a slight improvement 
Table 4.4: Comparison of passive $K_{i j}$ stiffness constants for non-zero values

\begin{tabular}{|c|cc|cc|cc|c|}
\hline$K_{i j}$ & \multicolumn{2}{|c|}{ VABS } & \multicolumn{2}{|c|}{ Bailey } & \multicolumn{2}{|c|}{ Present } & \% Difference with VABS \\
\hline$K_{11}$ & 8.477 & $E+05$ & 7.195 & $E+05$ & 8.519 & $E+05$ & 0.5 \\
$K_{12}$ & -1.794 & $E+03$ & -1.850 & $E+03$ & -1.850 & $E+03$ & 3.1 \\
$K_{13}$ & 6.337 & $E+02$ & -6.472 & $E+02$ & -6.472 & $E+02$ & -202.1 \\
$K_{22}$ & 1.278 & $E+02$ & 1.322 & $E+02$ & 1.322 & $E+02$ & 3.4 \\
$K_{23}$ & 4.461 & $E+01$ & 4.624 & $E+01$ & 4.624 & $E+01$ & 3.7 \\
$K_{33}$ & 9.567 & $E+01$ & 9.128 & $E+01$ & 9.818 & $E+01$ & 2.6 \\
$K_{44}$ & 2.070 & $E+02$ & 2.081 & $E+02$ & 2.094 & $E+02$ & 1.2 \\
\hline
\end{tabular}

from Bailey for the value of $K_{11}$. The difference in the values for $K_{13}$ can be explained by a difference in sign between the integral expressions, which is due to a correction in the present work.

\subsection{Blade Modelling}

In redesigning the inactive composite blade as a new smart blade with active fibre composites a two-celled box beam cross-section was first attempted. After this design had been studied, a more realistic blade design with an airfoil-shaped structural cross-section was then investigated. These two blade designs are discussed in further detail below; Table 4.5 summarizes the general blade characteristics taken from [57].

It should be noted that the airfoil selected for the two-celled blade design is the NACA 23012 airfoil, which is not a perfectly symmetric airfoil. This will result in small deviations from the structural and inertial formulations that are based on the assumption of perfect symmetry of the airfoil. The airfoil used for the two-celled box beam is NACA 0012, which is a symmetric airfoil. 
Table 4.5: Properties of scaled BO105 rotor blade

\begin{tabular}{|l|l|}
\hline rotor & BO105 hingeless \\
number of blades, $b$ & 4 \\
rotor radius, $L$ & $2 \mathrm{~m}$ \\
chord, $c$ & $0.121 \mathrm{~m}$ \\
airfoil & NACA 23012 \\
blade pre-twist, $\theta^{\prime}$ & 0 \\
solidity, $\sigma=b c / \pi r$ & 0.077 \\
tip speed & $218 \mathrm{~m} / \mathrm{sec}$ \\
lock number, $\gamma$ & 5.0 \\
elastic axis, e.a. & $25 \%$ chord \\
aerodynamic centre, a.c. & $25 \%$ chord \\
centre of gravity, c.g. & $25 \%$ chord \\
tension axis, t.a. & $25 \%$ chord \\
nominal rotational speed, $\Omega$ & $1040 \mathrm{rpm}(110 \mathrm{rad} / \mathrm{s})$ \\
nonrotating lead-lag frequency, $\omega_{v N R}$ & $9.00 \mathrm{~Hz}$ \\
nonrotating flap frequency, $\omega_{w N R}$ & $2.50 \mathrm{~Hz}$ \\
nonrotating torsional frequency, $\omega_{\phi N R}$ & $65.50 \mathrm{~Hz}$ \\
\hline
\end{tabular}

\subsubsection{Design Specifications}

The smart rotor concept is based on the fact that in either state when the piezoelectrics are active or inactive, the structural and inertial properties shall resemble those of a passive blade. A smart rotor whose actuators are either turned off or not working should still be required to perform as good as, or better than a passive rotor under stability concerns. Furthermore, one may expect better stability properties in a smart blade, but no deterioration in stability due to null actuation.

The designs of this thesis are based on a Mach-scaled version of the BO105 blade. Bielawa [4] states that there are four basic scaling conditions that should be met for blade design in order to retain the interactions of aerodynamic, elastic, inertial, and gravity forces. These are,

1. Frequency scaling: $\lambda_{f}=\frac{E}{m \Omega^{2}}$

2. Lock number: $\gamma=\frac{3 \rho a c L^{4}}{m}$ 
3. Advance ratio: $\mu=\frac{V}{\Omega L}$

4. Froude number: $F=\frac{\Omega^{2} L}{g}$ or

Mach number: $M=\frac{\Omega L}{a \infty}$

The first condition, frequency scaling, ensures that the rotor blades retain the correct natural frequencies due to structural bending characteristics as a function of the rotor hover frequency. Detailed expressions for these scaling ratios are [41],

$$
\frac{E I_{y^{\prime}}}{m \Omega^{2} L^{4}}=\frac{\left(\omega_{w N R} / \Omega\right)^{2}}{\beta_{1}^{4}}, \frac{E I_{z^{\prime}}}{m \Omega^{2} L^{4}}=\frac{\left(\omega_{v N R} / \Omega\right)^{2}}{\beta_{1}^{4}}, \frac{G J}{m \Omega^{2} k_{m}^{2} L^{2}}=\frac{\left(\omega_{\phi N R} / \Omega\right)^{2}}{\gamma_{1}^{2}}
$$

which describe the structural stiffness about the flap $\left(E I_{y^{\prime}}\right)$, lead-lag $\left(E I_{z^{\prime}}\right)$, and torsional $(G J)$ directions respectively, in relationship to the hover speed, $\Omega$, which provides centrifugal stiffening. These ratios are based on the exact closed-form solutions of mode equations for uniform nonrotating beams [58]. There is, however, no closed form solution for the case of a rotating beam, but standard results for a uniform vibrating beam indicate the rotational effects are small. For an untwisted cantilever beam a relationship between the rotating and nonrotating frequencies of a beam has been found experimentally [59]. Consequently, the nonrotating $\left(\omega_{N R}\right)$ frequencies for a pinned-free beam may be extrapolated and applied as above.

The Lock number, $\gamma$, which describes the blade mass ratio, not only restrains the mass, but also ensures that the rotor has correct aerodynamic damping and coupling characteristics. The advance ratio, $\mu$, does not apply here, but it describes the scaling of forward flight speed to rotor rotational speed. Finally, the fourth parameter accounts for either gravity effects (Froude-scaled) or compressibility effects (Mach-scaled) - whichever is desired.

In fulfilling design specifications of Table 4.5, the importance of some parameters must be outweighed by that of others. Specifically, the blade was designed more around attaining 
Table 4.6: Fixed properties for the BO105 blade

\begin{tabular}{|l|l|l|l|}
\hline mass per unit span, $m$ & $1.118 \mathrm{~kg} / \mathrm{m}$ & & \\
nonrotating lead-lag frequency, $\omega_{v N R} / \Omega$ & $0.514 / \mathrm{rev}$ & $\mathrm{EI}_{z^{\prime}}$ & $4623.9 \mathrm{Nm}^{2}$ \\
nonrotating flap frequency, $\omega_{w N R} / \Omega$ & $0.143 / \mathrm{rev}$ & $\mathrm{EI}_{y^{\prime}}$ & $357.9 \mathrm{Nm}^{2}$ \\
nonrotating torsional frequency, $\omega_{\phi N R} / \Omega$ & $3.741 / \mathrm{rev}$ & $\mathrm{GJ} / \mathrm{k}_{m}^{2}$ & $306810 \mathrm{~N}$ \\
\hline
\end{tabular}

correct structural stiffness $\left(E I_{y^{\prime}}, E I_{z^{\prime}}\right.$, and $\left.G J\right)$ than ensuring offsets between the quarterchord and the elastic and tension axes are zero. Ballast weights are used both to match the centre of gravity with the quarter-chord and to satisfy mass requirements, but do not contribute structurally. Table 4.6 outlines the first nonrotating frequencies for the BO105 scaled rotor and the resulting sectional mass - these become the fixed programmable parameters.

This summarizes the defining properties used in the designing of the passive blade structure. What follows is an outline of the two approaches undertaken.

\subsubsection{Two-Celled Box Beam}

This design concept, known as the two-celled box beam blade, consists of a box beam located entirely within a typical blade section. Figure 4.6 illustrates this configuration with the addition of leading and trailing edge lead ballast weights. The variables that describe the mass of the leading edge ballast, box beam section, and trailing edge ballast are $m_{1}, m_{2}$, and $m_{3}$ located respectively at distances to their respective centres of gravity $s_{1}, s_{2}$, and $s_{3}$, measured from the nose of the airfoil. The box beam size determines the mass distribution between the ballasts $m_{1}$ and $m_{3}$ with radii $r_{1}$ and $r_{3}$.

For this two-celled box beam, twisting actuation moments are assumed to be transmitted to the outer beam section through some mechanism. Although this type of concept helps to simplify the cross-sectional constants of Chapter 2, it does provide disadvantages of manufacturing an enclosed inaccessible structure, and defining the load-transferring 


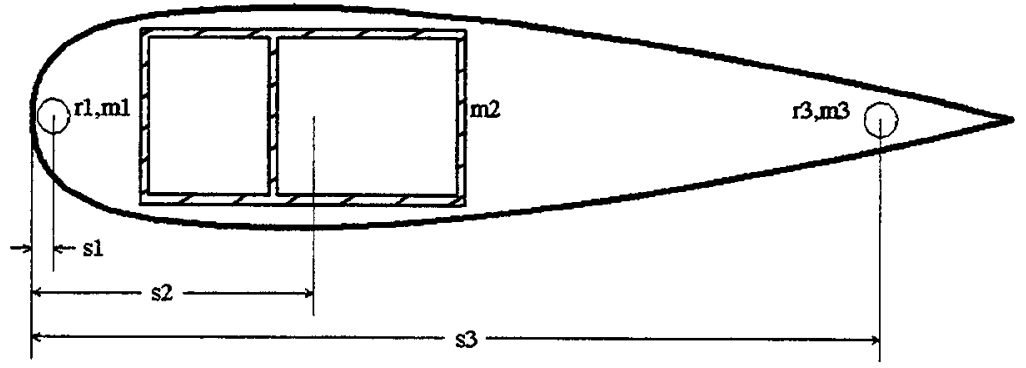

Figure 4.6: Two-celled box beam blade

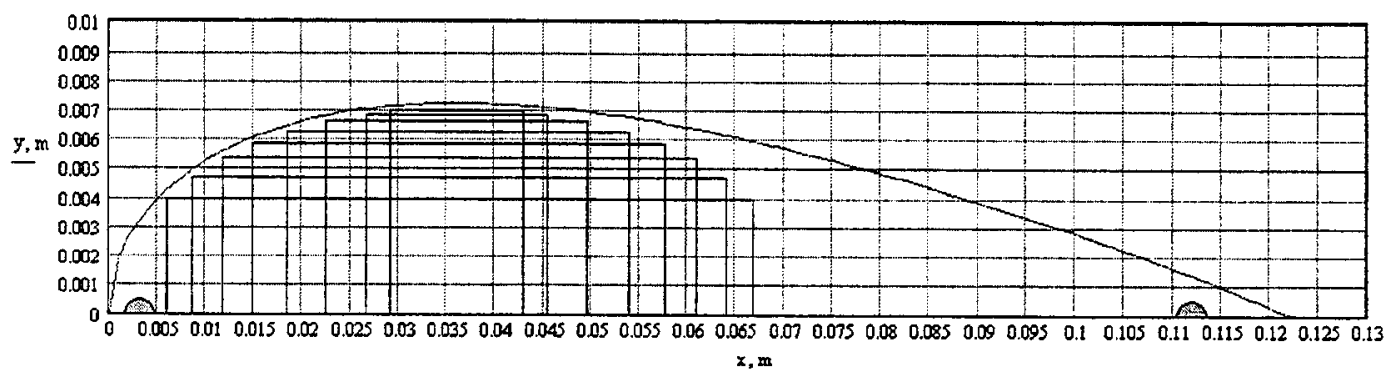

Figure 4.7: NACA 0012 blade section illustrating various box beam sizes

mechanism. Simplified expressions of the stiffness constant integrals pertaining to the box beam design are found in Appendix $\mathrm{H}$.

Figure 4.7 illustrates possible renditions of the box beam enclosed within the NACA 0012 airfoil. By varying the height and width of the box beam convergence of a solution to achieve those fixed blade properties in Table 4.6 may be found. Widths of box beams varied from $0.01 \mathrm{~m}$ to $0.08 \mathrm{~m}$; the height varied between a minimum of $0.004 \mathrm{~m}$ and a maximum of $0.014 \mathrm{~m}$. This figure also shows the locations of the ballasts weights.

Five sets of designs were carried out by varying the number of layers, ply orientations, length and height of the box beam, web and ballast locations. These simulations are summarized in Table 4.7. The designs were constrained by asking the following questions:

1. Does the box fit in the NACA section?

2. Are the ballast weights located between the boundaries of the box beam and the 
NACA section?

3. Is the error between the required and the resulting $E I_{y^{\prime}}, E I_{z^{\prime}}$, and $G J / k_{m}^{2}$ stiffness values less than $10-15 \%$ ?

Of the five simulations, only the first one gave results that partially satisfied the above constraints. However the errors were too high to be satisfactory and the box beam did not fit inside the bounds of the NACA airfoil.

Lack of results under these simulations warranted a new approach in design. A twocelled airfoil section was therefore pursued.

\subsubsection{Two-Celled Blade}

In comparison with the box beam section, the two-celled blade section is a much more feasible and realistic design. Although it is slightly more difficult to approximate the various sectional characteristics such as the moments of inertia, this design represents the actual blade shape. Figure 4.8a illustrates the two-celled blade design with actuators. Figure $4.8 \mathrm{~b}$ illustrates the approximation method used in calculating sectional characteristics by dividing up the airfoil into elements along the chord-wise direction.

The same constraints as those of the box beam design were imposed on this blade section. Again, an error of $10-15 \%$ deviation from stiffness for $E I_{y^{\prime}}, E I_{z^{\prime}}$, and $G J / k_{m}^{2}$, is desired. Much more success was attained with the airfoil cross-section designs with variations in web position, ply orientation, and number of layers for various sides.

Seven sets of passive blade designs with CUS-style configurations were tested. Table 4.8 lists these seven passive blade designs where ' $L E$ ' and ' $T E$ ' refer to the leading edges and trailing edges, respectively. Plies are located heavily at the leading edge of the crosssection rather than the trailing edge in all designs; with the addition of active fibres this will provide the large moment arm needed for maximum twist authority. It would also 
Table 4.7: Box beam designs

\begin{tabular}{|c|c|c|c|}
\hline & Design Specifications & Ply Orientations & Total Thickness \\
\hline \multicolumn{4}{|l|}{ 1st run } \\
\hline $\begin{array}{l}\text { material } \\
\text { top } \\
\text { bottom } \\
\text { left vertical } \\
\text { web } \\
\text { right vertical } \\
\text { length range } \\
\text { height range } \\
\text { web range }\end{array}$ & $\begin{array}{c}\text { AS4 } / 3501-6 \\
\text { no. layers } \\
8 \\
8 \\
8 \\
8 \\
8 \\
0.01 \text { to } 0.08 \mathrm{~m} \\
0.004 \text { to } 0.014 \mathrm{~m} \\
10 \% \text { to } 90 \% \text { of chord }\end{array}$ & $\begin{array}{l}{[0 / 90 / 45 / 45 /-45 /-45 / 0 / 90]} \\
{[0 / 90 / 45 / 45 /-45 /-45 / 0 / 90]} \\
{[0 / 90 / 45 / 45 /-45 /-45 / 0 / 90]} \\
{[0 / 90 / 45 / 45 /-45 /-45 / 0 / 90]} \\
{[0 / 90 / 45 / 45 /-45 /-45 / 0 / 90]}\end{array}$ & $\begin{array}{l}0.001016 \mathrm{~m} \\
0.001016 \mathrm{~m} \\
0.001016 \mathrm{~m} \\
0.001016 \mathrm{~m} \\
0.001016 \mathrm{~m}\end{array}$ \\
\hline $\begin{array}{l}\text { results: } \\
\text { difference from } \mathrm{EIy} \\
\text { difference from } \mathrm{EIz} \\
\text { difference from } \mathrm{GJ} / \mathrm{k}_{m}^{2}\end{array}$ & \multicolumn{3}{|l|}{$\begin{array}{l}<160(45 \%) \\
\text { no constraint } \\
<30700(10 \%) \\
\end{array}$} \\
\hline solution convergence? & \multicolumn{3}{|l|}{ none } \\
\hline \multicolumn{4}{|l|}{ 2nd run } \\
\hline $\begin{array}{l}\text { material } \\
\text { top } \\
\text { bottom } \\
\text { left vertical } \\
\text { web } \\
\text { right vertical }\end{array}$ & $\begin{array}{c}\text { AS4/3501-6 } \\
\text { no. layers } \\
8 \\
8 \\
12 \\
12 \\
12 \\
\end{array}$ & $\begin{array}{c}{[0 / 90 / 45 / 45 /-45 /-45 / 0 / 90]} \\
{[0 / 90 / 45 / 45 /-45 /-45 / 0 / 90]} \\
{\left[0 / 45 /-45 / 90 / 45_{2} /-45_{2} / 0 / 45 /-45 / 90\right]} \\
{\left[0 / 45 /-45 / 90 / 45_{2} /-45_{2} / 0 / 45 /-45 / 90\right]} \\
{\left[0 / 45 /-45 / 90 / 45_{2} /-45_{2} / 0 / 45 /-45 / 90\right]}\end{array}$ & $\begin{array}{l}0.001016 \mathrm{~m} \\
0.001016 \mathrm{~m} \\
0.001524 \mathrm{~m} \\
0.001524 \mathrm{~m} \\
0.001524 \mathrm{~m}\end{array}$ \\
\hline results: & \multicolumn{3}{|c|}{ 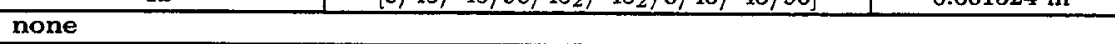 } \\
\hline \multicolumn{4}{|l|}{ 3rd run } \\
\hline $\begin{array}{l}\text { material } \\
\text { top } \\
\text { bottom } \\
\text { left vertical } \\
\text { web } \\
\text { right vertical }\end{array}$ & $\begin{array}{c}\text { AS4/3501-6 } \\
\text { no. layers } \\
12 \\
12 \\
8 \\
8 \\
8 \\
\end{array}$ & $\begin{array}{c}{\left[0 / 45 /-45 / 90 / 45_{2} /-45_{2} / 0 / 45 /-45 / 90\right]} \\
{\left[0 / 45 /-45 / 90 / 45_{2} /-45_{2} / 0 / 45 /-45 / 90\right]} \\
{[0 / 90 / 45 / 45 /-45 /-45 / 0 / 90]} \\
{[0 / 90 / 45 / 45 /-45 /-45 / 0 / 90]} \\
{[0 / 90 / 45 / 45 /-45 /-45 / 0 / 90]}\end{array}$ & $\begin{array}{l}0.001524 \mathrm{~m} \\
0.001524 \mathrm{~m} \\
0.001016 \mathrm{~m} \\
0.001016 \mathrm{~m} \\
0.001016 \mathrm{~m}\end{array}$ \\
\hline results: & \multicolumn{3}{|c|}{ 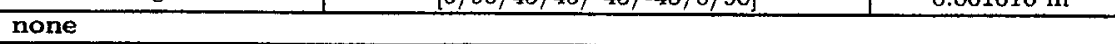 } \\
\hline \multicolumn{4}{|l|}{ 4th run } \\
\hline $\begin{array}{l}\text { material } \\
\text { top } \\
\text { bottom } \\
\text { left vertical } \\
\text { web } \\
\text { right vertical }\end{array}$ & $\begin{array}{c}\text { AS4/3501-6 } \\
\text { no. layers } \\
8 \\
8 \\
12 \\
12 \\
12 \\
\end{array}$ & $\begin{array}{c}{[0 / 90 / 45 / 45 /-45 /-45 / 0 / 90]} \\
{[0 / 90 / 45 / 45 /-45 /-45 / 0 / 90]} \\
{[0 / 30 / 60 / 90 / 45 / 45 /-45 /-45 / 0 /-60 /-30 / 90]} \\
{[0 / 30 / 60 / 90 / 45 / 45 /-45 /-45 / 0 /-60 /-30 / 90]} \\
{[0 / 30 / 60 / 90 / 45 / 45 /-45 /-45 / 0 /-60 /-30 / 90]}\end{array}$ & $\begin{array}{l}0.001016 \mathrm{~m} \\
0.001016 \mathrm{~m} \\
0.001524 \mathrm{~m} \\
0.001524 \mathrm{~m} \\
0.001524 \mathrm{~m}\end{array}$ \\
\hline results: & \multicolumn{3}{|c|}{ 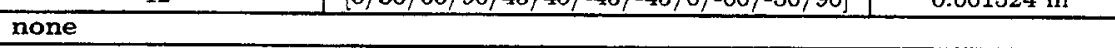 } \\
\hline \multicolumn{4}{|l|}{5 th run } \\
\hline $\begin{array}{l}\text { material } \\
\text { top } \\
\text { bottom } \\
\text { left vertical } \\
\text { web } \\
\text { right vertical } \\
\end{array}$ & $\begin{array}{c}\text { AS4/3501-6 } \\
\text { no. layers } \\
12 \\
12 \\
12 \\
12 \\
12 \\
\end{array}$ & $\begin{array}{l}{[0 / 30 / 60 / 90 / 45 / 45 /-45 /-45 / 0 / 30 / 60 / 90]} \\
{[0 / 30 / 60 / 90 / 45 / 45 /-45 /-45 / 0 / 30 / 60 / 90]} \\
{[0 / 30 / 60 / 90 / 45 / 45 /-45 /-45 / 0 / 30 / 60 / 90]} \\
{[0 / 30 / 60 / 90 / 45 / 45 /-45 /-45 / 0 / 30 / 60 / 90]} \\
{[0 / 30 / 60 / 90 / 45 / 45 /-45 /-45 / 0 / 30 / 60 / 90]} \\
\end{array}$ & $\begin{array}{l}0.001524 \mathrm{~m} \\
0.001524 \mathrm{~m} \\
0.001524 \mathrm{~m} \\
0.001524 \mathrm{~m} \\
0.001524 \mathrm{~m} \\
\end{array}$ \\
\hline results: & \multicolumn{3}{|l|}{ none } \\
\hline
\end{tabular}




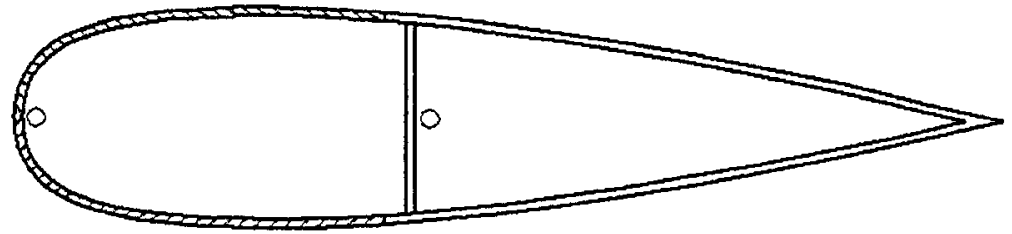

(a)

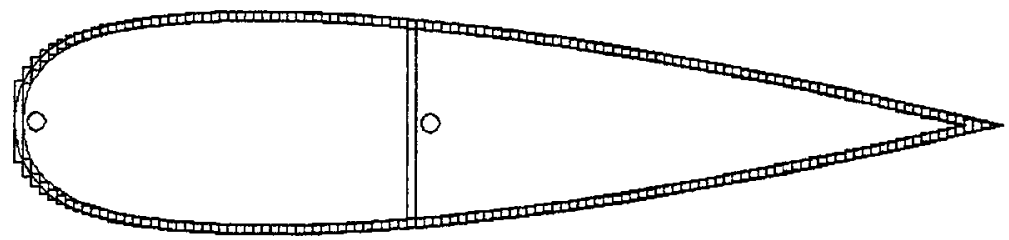

(b)

Figure 4.8: Two-celled blade design with a) actuator location and b) sectioning for parameter approximation

be plausible to locate more plies at the trailing edge, or fairing section, but the resulting stiffness would need to be studied. Moreover, adding unnecessary AFCs will also raise the costs.

\section{Web Position}

The results between desired and designed stiffness, $E I_{y^{\prime}}, E I_{z^{\prime}}$, and $G J / k_{m}^{2}$ are plotted as a function of the web position in Figures 4.9, 4.10, and 4.11. This gives an indication of both the best web location and the best design. As well, a statistical analysis with weighting values assigned to each design was performed. These weighting values indicate either a good (1) or poor (7) match depending on resulting stiffness values and centroidal position for each web location. Comparison between these three graphs, along with the statistical results, indicates that Designs 1 and 7 give the least amount of deviation from the specified stiffness values. These figures also indicate that the web position should be roughly between $45-60 \%$ of the chord length from the leading edge. 
Table 4.8: Ply orientations for passive blade designs

\begin{tabular}{|l|l|l|}
\hline & Set 1 & Set 5 \\
top \& bottom LE & {$[0 / 90 / 45 / 45 /-45 /-45 / 0 / 90]$} & {$[0 / 90 / 45 / 45 /-45 /-45 / 0 / 90]$} \\
web & {$[0 / 90 / 0 / 90]$} & {$[0 / 90 / 0 / 90]$} \\
top \& bottom TE & {$[0 / 90]$} & {$[0 / 45 /-45 / 0]$} \\
\hline & Set 2 & Set 6 \\
top \& bottom LE & {$[0 / 90 / 45 / 45 / 45 /-45 /-45 /-45 / 0 / 90]$} & {$[0 / 45 / 45 / 45 /-45 /-45 /-45 / 0]$} \\
web & {$[0 / 90 / 0 / 90]$} & {$[0 / 90 / 45 / 45 / 0 / 90]$} \\
top \& bottom TE & {$[0 / 90]$} & {$[0 / 90]$} \\
\hline & Set 3 & Set 7 \\
top \& bottom LE & {$[0 / 90 / 45 / 45 /-45 /-45 / 0 / 90]$} & {$[0 / 45 / 30 / 60 / 45 / 30 / 60 / 0]$} \\
web & {$[0 / 90 / 45 / 45 / 0 / 90]$} & {$[0 / 90 / 45 / 45 / 0 / 90]$} \\
top \& bottom TE & {$[0 / 90]$} & {$[0 / 90]$} \\
\hline \multirow{2}{*}{$\begin{array}{l}\text { top \& bottom LE } \\
\text { web }\end{array}$} & {$[0 / 45 / 45 /-45 /-45 / 0]$} & \\
top \& bottom TE & {$[0 / 90 / 0 / 90]$} & \\
\hline
\end{tabular}

Comparison of Web Position with Ely' for Passive Two-Celled Blade

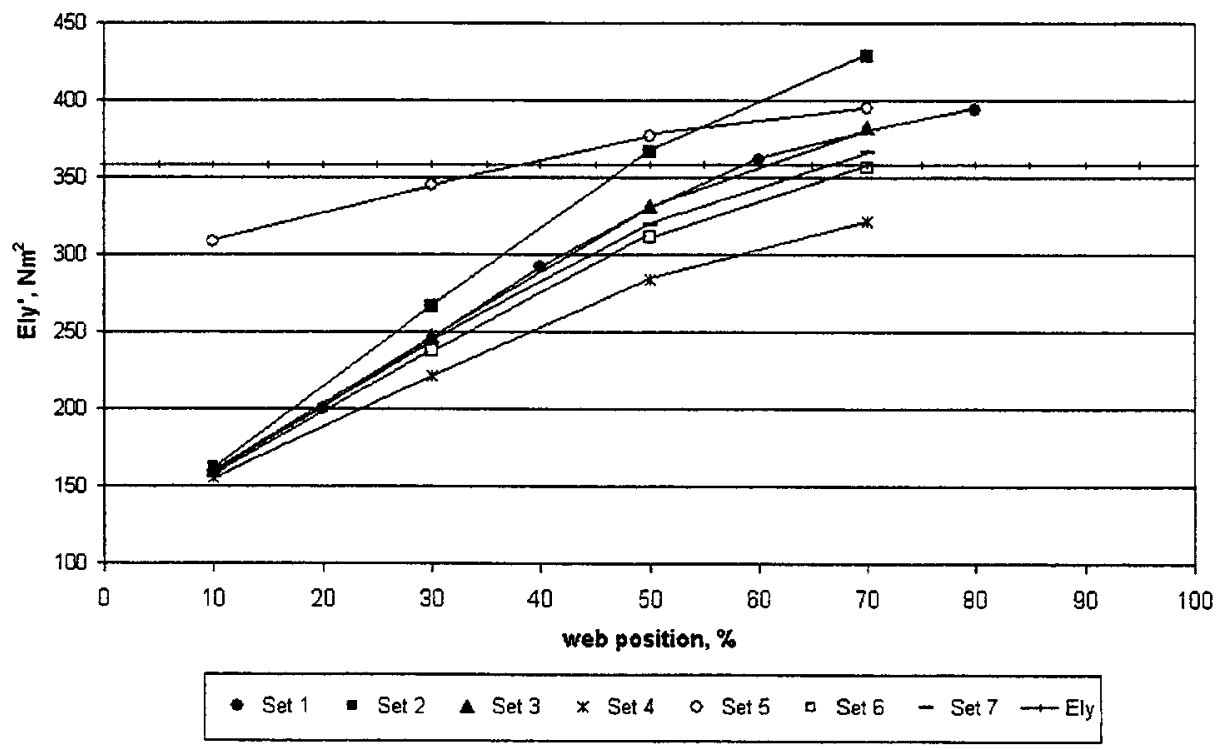

Figure 4.9: Relationship between web position and stiffness in flap direction 


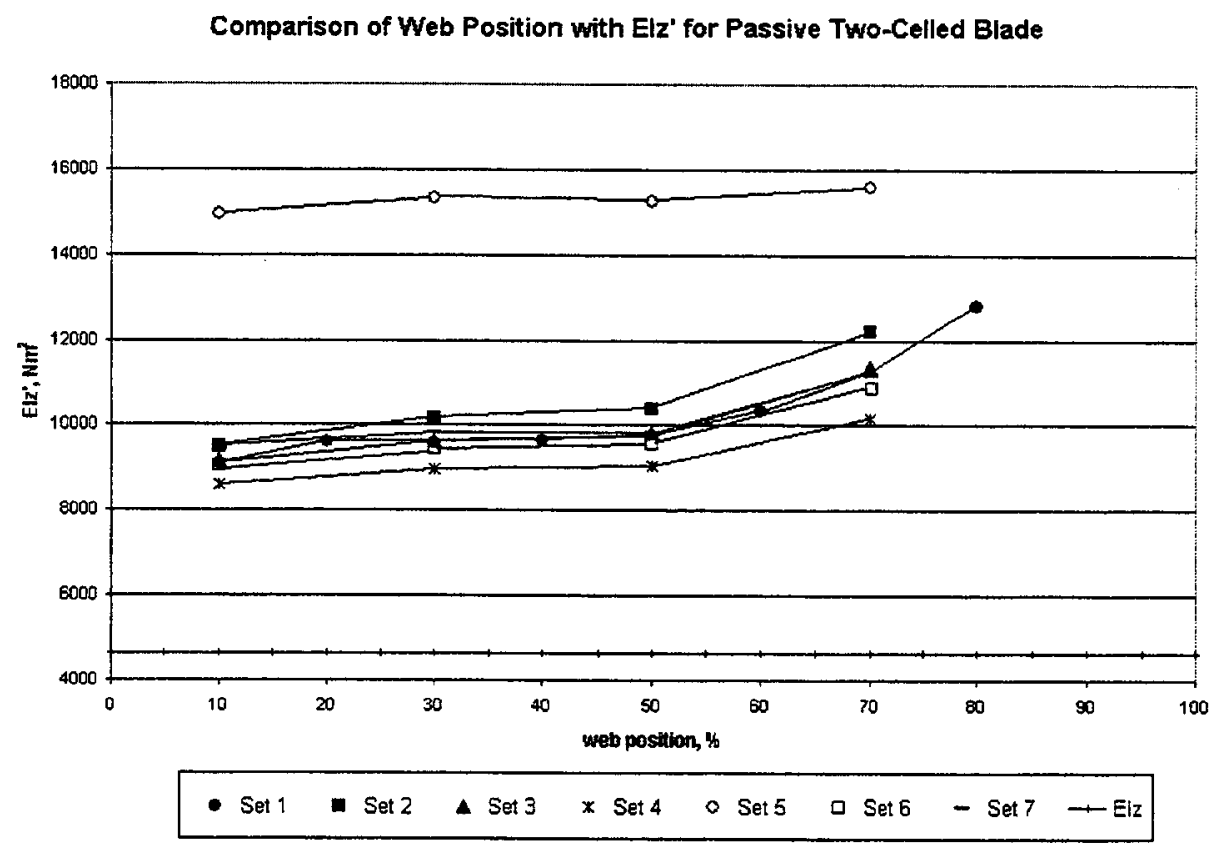

Figure 4.10: Relationship between web position and stiffness in lead-lag direction

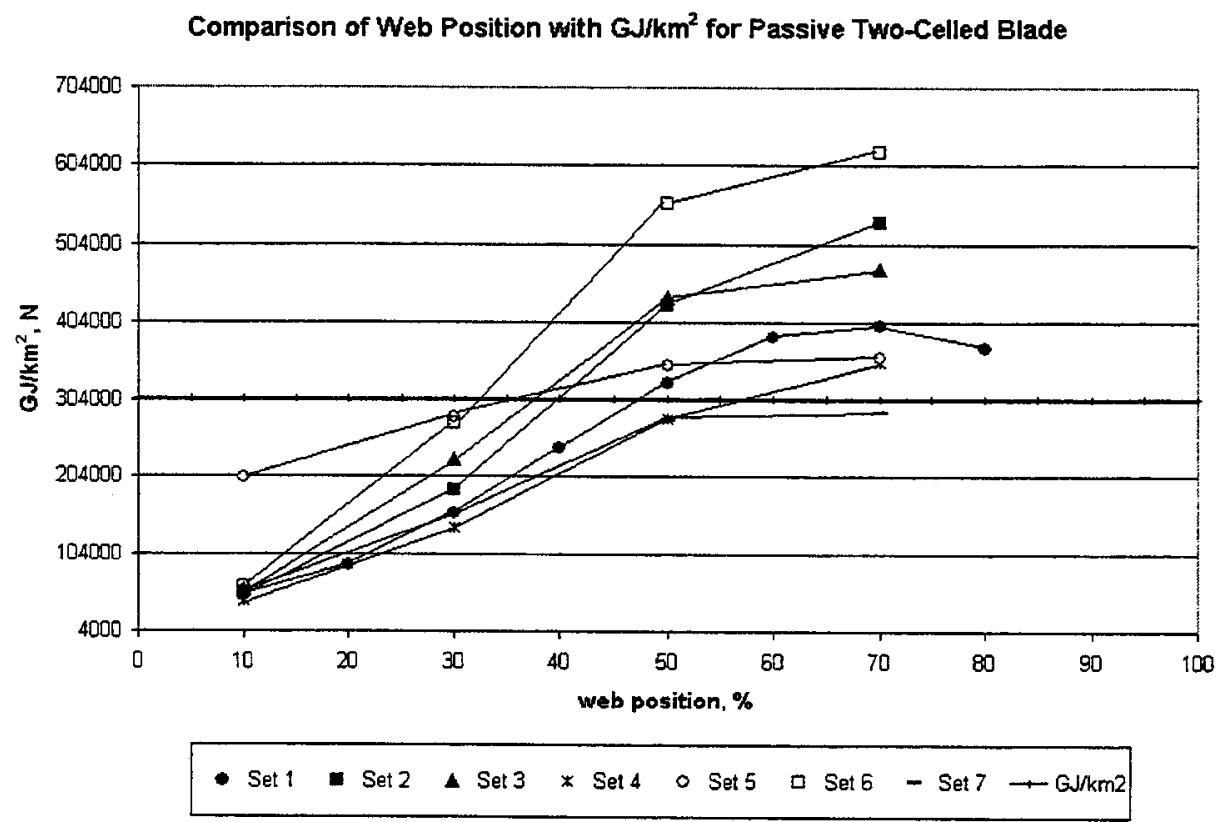

Figure 4.11: Relationship between web position and torsional stiffness 
Table 4.9: Properties of Design 8

\begin{tabular}{|l|l|}
\hline top \& bottom LE & {$[0 / 90 / 45 / 45 / 45 / 45 / 0 / 90]$} \\
web & {$[0 / 90 / 45 / 45 / 0 / 90]$} \\
top \& bottom TE & {$[0 / 90]$} \\
\hline
\end{tabular}

\section{Refinement and Ballast Location}

Since the torsional stiffness parameter, $G J / k_{m}^{2}$, is a function of the mass per unit length of the beam, $m$, this value is therefore also dependent upon the ballast masses and locations. Designs 1 and 7 may be further refined in consideration of the ballasts. At this point one other design is introduced to act as a comparison with the 1st design. Hereafter this final design will be referred to as Design Case 8. Its properties are summarized in Table 4.9.

For manufacturing purposes the leading edge ballast should be in close proximity to the nose, and the trailing edge ballast located directly behind or in front of the web. In placing the trailing edge ballast at the rearmost end of the airfoil section one looses accuracy with the stiffness values.

The three finalized design sections are given in Figures 4.12, 4.13, and 4.14. Sides pertaining to the top and bottom leading edge sections, the web, and the bottom and top trailing edge sections are denoted by $1,2,3,4$, and 5 respectively. The greatest amount of error between the desired and the resulting stiffness values lay in the lead-lag direction $\left(E I_{z^{\prime}}\right)$, by a factor of 2 as indicated in Table 4.10. Recall that Table 4.6 summarizes the desired stiffness values. The flapping stiffness and torsional stiffness were both in very good agreement.

Errors between designed and required stiffness values are also in good agreement with published results. Shin, for a design resembling the 1st design had similar magnitudes of error. Refer to Table 4.11 for his results [37].

With the final three concepts for the actuated helicopter blade chosen, the stability and perturbation/response analyses can now be carried out. 
Table 4.10: Characteristics of three final blade designs

\begin{tabular}{|c|c|c|c|}
\hline \multicolumn{4}{|c|}{ Design 1} \\
\hline $\begin{array}{c}\text { Mset, } \mathrm{kg} / \mathrm{m} \\
\mathrm{EI}_{y}, \mathrm{Nm}^{2} \\
\mathrm{EI}_{y} \text { difference, } \mathrm{Nm}^{2} \\
\text { web position, \% }\end{array}$ & $\begin{array}{c}\mathrm{m} 2, \mathrm{~kg} / \mathrm{m} \\
\mathrm{EI}_{z}, \mathrm{Nm}^{2} \\
\mathrm{EI}_{z} \text { difference, } \mathrm{Nm}^{2}\end{array}$ & $\begin{array}{c}\mathrm{m} 1, \mathrm{~kg} / \mathrm{m} \\
\mathrm{GJ} / \mathrm{k}_{m}^{2}, \mathrm{~N} \\
\mathrm{GJ} / \mathrm{k}_{m}^{2} \text { difference, } \mathrm{N} \\
\text { centroid position, \% }\end{array}$ & centroid error, $\%$ \\
\hline 1.118 & 0.271 & 0.469 & 0.378 \\
\hline 312.3 & 9696.3 & 299470 & \\
\hline 45.6 & 5072.4 & 7330 & \\
\hline 45 & & 34 & 9.5 \\
\hline $\begin{array}{c}\mathrm{EI}_{y} \text { error, \% } \\
12.7 \\
\end{array}$ & $\begin{array}{c}\mathrm{EI}_{z} \text { error, \% } \\
109.7 \\
\end{array}$ & $\begin{array}{c}\mathrm{GJ} / \mathrm{k}_{m}^{2} \text { error, \% } \\
2.4 \\
\end{array}$ & \\
\hline \multicolumn{4}{|c|}{ Design 7} \\
\hline $\begin{array}{c}\text { Mset, } \mathrm{kg} / \mathrm{m} \\
\mathrm{EI}_{y}, \mathrm{Nm}^{2} \\
\mathrm{EI}_{y} \text { difference, } \mathrm{Nm}^{2} \\
\text { web position, \% }\end{array}$ & $\begin{array}{c}\mathrm{m} 2, \mathrm{~kg} / \mathrm{m} \\
\mathrm{EI}_{z}, \mathrm{Nm}^{2} \\
\mathrm{EI}_{z} \text { difference, } \mathrm{Nm}^{2}\end{array}$ & $\begin{array}{c}\mathrm{m} 1, \mathrm{~kg} / \mathrm{m} \\
\mathrm{GJ} / \mathrm{k}_{m}^{2}, \mathrm{~N} \\
\mathrm{GJ} / \mathrm{k}_{m}^{2} \text { difference, } \mathrm{N} \\
\text { centroid position, \% }\end{array}$ & centroid error, $\%$ \\
\hline 1.118 & 0.292 & 0.578 & 0.248 \\
\hline 319.8 & 9832.2 & 221670 & \\
\hline 38.1 & 5208.2 & 85140 & \\
\hline 50 & & 36 & 10.6 \\
\hline $\begin{array}{c}\mathrm{EI}_{y} \text { error, \% } \\
10.6\end{array}$ & $\begin{array}{c}\mathrm{EI}_{z} \text { error, } \% \\
112.6\end{array}$ & $\begin{array}{c}\mathrm{GJ} / \mathrm{k}_{m}^{2} \text { error, } \% \\
27.7\end{array}$ & \\
\hline \multicolumn{4}{|c|}{ Design 8} \\
\hline $\begin{array}{c}\text { Mset, } \mathrm{kg} / \mathrm{m} \\
\mathrm{EI}_{y}, \mathrm{Nm}^{2} \\
\mathrm{EI}_{y} \text { difference, } \mathrm{Nm}^{2} \\
\text { web position, \% }\end{array}$ & $\begin{array}{c}\mathrm{m} 2, \mathrm{~kg} / \mathrm{m} \\
\mathrm{EI}_{z}, \mathrm{Nm}^{2} \\
\mathrm{EI}_{z} \text { difference, } \mathrm{Nm}^{2}\end{array}$ & $\begin{array}{c}\mathrm{ml}, \mathrm{kg} / \mathrm{m} \\
\mathrm{GJ} / \mathrm{k}_{m}^{2}, \mathrm{~N} \\
\mathrm{GJ} / \mathrm{k}_{m}^{2} \text { difference, } \mathrm{N} \\
\text { centroid position, \% }\end{array}$ & centroid error, \% \\
\hline 1.118 & 0.307 & 0.559 & 0.251 \\
\hline 302.7 & 9566.2 & 321320 & \\
\hline 55.2 & 4942.3 & 14510 & \\
\hline 55 & & 37 & 11.7 \\
\hline $\begin{array}{c}\mathrm{EI}_{y} \text { error, \% } \\
15.4\end{array}$ & $\begin{array}{c}\mathrm{EI}_{z} \text { error, } \% \\
106.9 \\
\end{array}$ & $\begin{array}{c}\mathrm{GJ} / \mathrm{k}_{m}^{2} \text { error, } \% \\
4.7 \\
\end{array}$ & \\
\hline
\end{tabular}




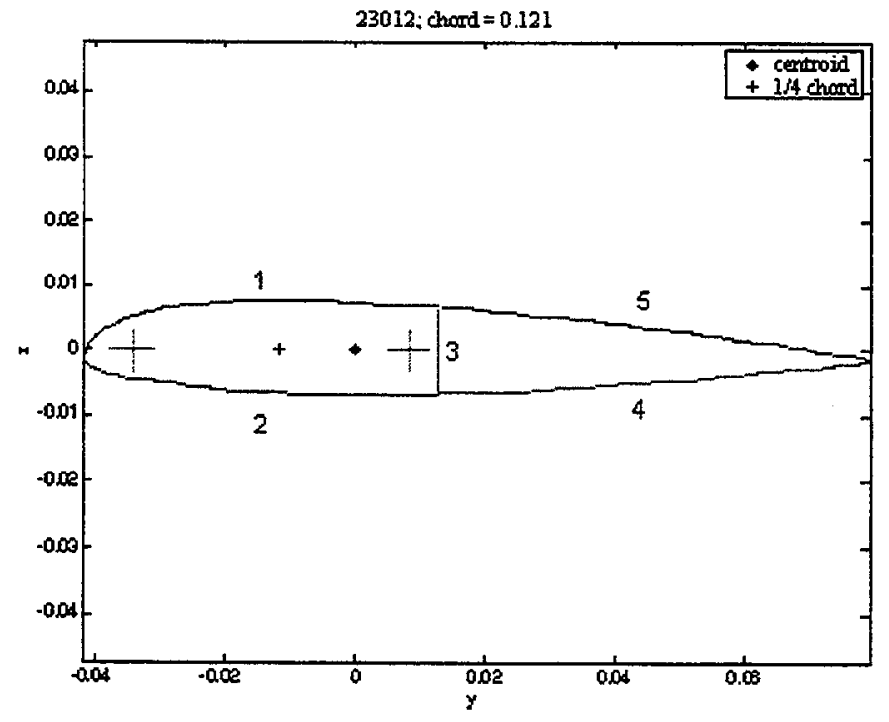

Figure 4.12: Final concept no. 1

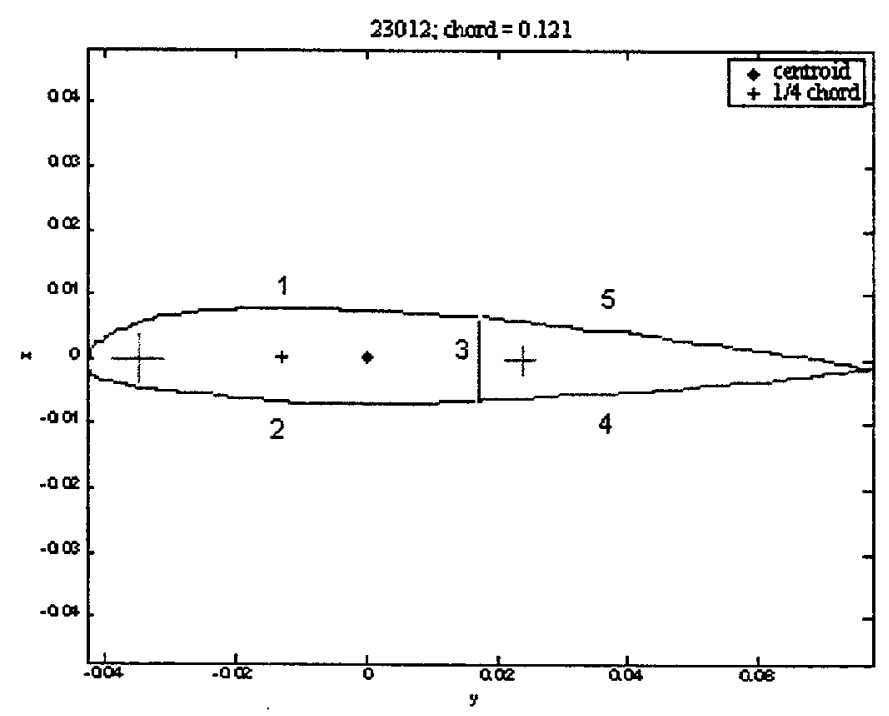

Figure 4.13: Final concept no. 7 


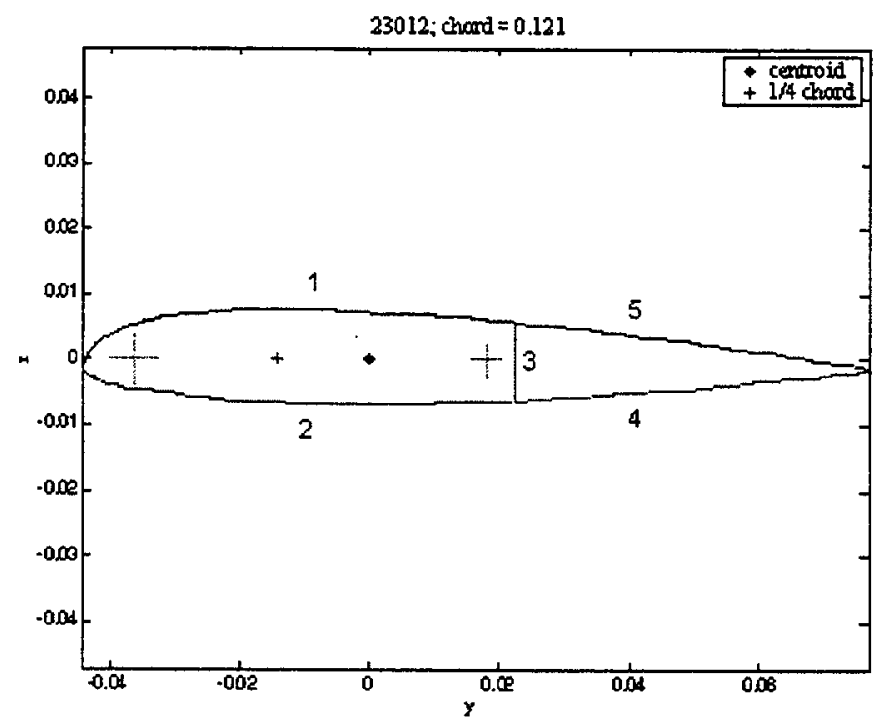

Figure 4.14: Final concept no. 8

Table 4.11: Result of final design concept for Shin

\begin{tabular}{|l|l|}
\hline Side & Orientation \\
\hline Nose & {$[0 / 90 / 0 / 45 / 45 /-45 /-45 / 0 / 90]$} \\
Active Region (fore section) & {$[0 / 90 / 45 / 45 /-45 /-45 / 0 / 90]$} \\
Web & {$[0 / 90 / 0 / 90]$} \\
Fairing (aft section) & {$[0 / 90]$} \\
\hline stiffness & error from requirement \\
\hline $\mathrm{GJ}_{\mathrm{Nm}} \mathrm{Nm}^{2}$ & $-25.3 \%$ \\
$\mathrm{EI}_{\text {flap }}, \mathrm{Nm}^{2}$ & $-7.1 \%$ \\
$\mathrm{EI}_{\text {lag }}, \mathrm{Nm}^{2}$ & $-84.4 \%$ \\
\hline
\end{tabular}




\section{Chapter 5}

\section{Stability and Response}

The focus of this chapter is in proving that a helicopter rotor blade equipped with smart materials is as stable as one without, and that it will be able to respond to external loads in a stable manner. This will be achieved in two ways. First, the lead-lag, flap, and torsional frequencies of the smart blade will be compared with those of the non-actuated or baseline blade. Secondly, as an introduction to the control law which will define the blade system, it will be shown that the blade will respond in a favourable manner to impulsive actuation.

Chapter 3 introduced the steady-state and perturbation equations that describe the dynamics of a helicopter rotor blade in hover. They will be applied here. In essence, the defiected blade in hover, illustrated in Figure 3.2, is defined by these steady-state and perturbation components given in Eq. (3.40). The steady-state equilibrium deflections, referred to as the trim conditions, describe the operating state of the rotor blade and also become the coefficients of the perturbation equations. A sudden perturbation, represented by the addition of an external function $u(t)$ on the right hand side of the equation of motion, Eq. (3.44), will be counteracted by the force from the piezoelectrics, $\{F\}$. All three of the blade designs of Chapter 4 are evaluated by obtaining their equilibrium deflections and investigating their stability as a function of pitch angle, $\theta$, and length of blade actuated. 


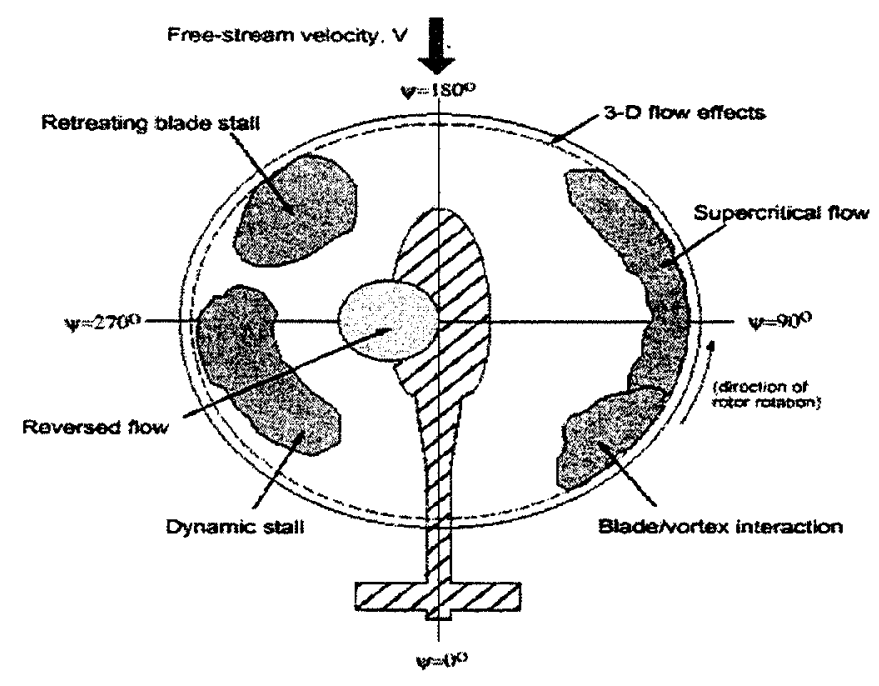

Figure 5.1: Unsteady aerodynamic loads experienced by main rotor

There are three general conditions that a smart blade might work under:

1. AFCs turned off (denoted hereafter as the 'zero-actuation' case)

2. AFCs turned on through a constant DC current

3. AFCs turned on through an $\mathrm{AC}$ current

All results will be compared against the conventional composite baseline blade. Consider that a helicopter blade is subject to different external loads at various azimuths throughout its rotation as illustrated in Figure 5.1 [37]. Application of an AC current will provide periodic actuation as the rotors undergo vibrations from these external loads.

Stability is typically described by the eigenvalues of a set of homogeneous equations, which for this application are the $3 \mathrm{~N}$ linearized perturbation equations for $v, w$, and $\phi$. These eigenvalues, $\lambda_{j}$, represent natural modes of vibration of the system, and are denoted as complex numbers,

$$
\lambda_{j}=\sigma_{j} \pm i \omega_{j}
$$

where the real part, $\sigma_{j}$, describes the growth or decay of the corresponding mode as a 
function of time, and the imaginary part, $i \omega_{j}$, denotes the frequency of the corresponding mode. A negative real part denotes an asymptotically stable mode. From a theoretical point of view, the stability of the system requires all its modes to be stable. However, for a continuous system such as a rotor blade with infinite number of modes, the first few modes associated with each type of action, i.e. flap, lead-lag, and torsion, are of primary interest and hence are investigated in the present work. It should be noted that depending on the values of $\sigma_{j}$ and $i \omega_{j}$ two types of instabilities may occur in lifting surfaces. These are flutter and divergence. Flutter is characterized as a dynamic instability in flight. A positive $\sigma_{j}$ for any mode indicates a flutter type instability. This corresponds to the presence of a root on the right hand side of the Laplace domain. Divergence is a static instability and is only dependent upon the elasticity of the lifting surface. It is characterized by an eigenvalue with a positive real part, but zero imaginary part. This corresponds to a root on the real axis of the right hand side of the Laplace domain.

A second validation study is carried out to verify the code for obtaining the eigenvalues. The example is one that was carried out by Hodges and Ormiston in obtaining the leadlag, flap, and torsional frequencies of a passive blade. Once confirmed, the respective frequencies for all three blade designs may then be found. Eigenvalues for the smart blade under a DC load may be found by directly solving the homogeneous equation, whereas the Floquet method is used in solving the time-dependent homogeneous equation resulting from an applied AC current.

The closed form transfer function for Design 8 is presented last to introduce the feedback-response system that will eventually tie together the functionality of the blade system in future studies. 
Table 5.1: Plies on leading edge surface replaced by active plies in italic

\begin{tabular}{|c|c|c|}
\hline Design & No. plies & Plies replaced by active plies \\
\hline $1 \mathrm{a}$ & 2 plies active & {$\left[\begin{array}{llllllll}0 & 90 & 45 & 45 & -45 & -45 & 0 & 90\end{array}\right]$} \\
\hline Ib & 4 plies active & {$\left[\begin{array}{llllllll}0 & 90 & 45 & 45 & -45 & -45 & 0 & 90\end{array}\right]$} \\
\hline $7 \mathrm{a}$ & 2 plies active & {$\left[\begin{array}{llllllll}0 & 45 & 30 & 60 & 45 & 30 & 60 & 0\end{array}\right]$} \\
\hline $7 \mathrm{~b}$ & 4 plies active & {$\left[\begin{array}{llllllll}0 & 45 & 30 & 60 & 45 & 30 & 60 & 0\end{array}\right]$} \\
\hline $8 a$ & 2 plies active & {$\left[\begin{array}{llllllll}0 & 90 & 45 & 45 & 45 & 45 & 0 & 90\end{array}\right]$} \\
\hline $8 \mathrm{~b}$ & 4 plies active & {$\left[\begin{array}{llllllll}0 & 90 & 45 & 45 & 45 & 45 & 0 & 90\end{array}\right]$} \\
\hline
\end{tabular}

\subsection{Case Studies}

In Chapter 4, the three blade designs 1, 7, and 8, were selected as final design alternatives. An active fibre composite (AFC) blade can be obtained for these designs by replacing some of the graphite-epoxy plies in the leading edge with the active fibre composite material. For each design alternative, two different AFC blades were considered, one with 2 active layers and the other with 4 active layers. This results in six AFC design cases as given in Table 5.1. The stability of each blade design under various actuation schemes is investigated and the results are compared against the baseline blade.

As was outlined in Chapter 4, the baseline blade only contains the graphite-epoxy composite AS4/3501-6. This baseline blade exhibits the natural frequencies and stability characteristics of a typical BO105 helicopter rotor blade. The smart blades are modelled from this by replacing some of the graphite-epoxy layers with the active fibre composite material. Table 5.2 lists the material properties for an AFC ply utilizing a PZT-5H type piezoelectric. The applied voltage is given in Table 5.2 as D3. Du Plessis and Hagood tested a static model for tip twist as a function of applied voltage for values up to 2000 $\mathrm{V}$ [28]. At $1800 \mathrm{~V}$ they achieved degrees of twist on the order of $1.3^{\circ}$. Using this as a model, this voltage is applied to all three designs. The piezoelectric electromechanical coupling coefficients are given by $d_{e L L}, d_{e L T}$, and $d_{e T T}$. Subscripts $L$ and $T$ correspond to the longitudinal and transverse directions of this transversely isotropic piezoelectric fibre. 
Table 5.2: Material properties of AFC with the piezoelectric PZT-5H

\begin{tabular}{|l|l|}
\hline$E_{L L}$ & $30.18 E+09 \mathrm{~Pa}$ \\
$E_{N N}=E_{L N}$ & $14.9 E+09 \mathrm{~Pa}$ \\
$G_{L T}=G_{L N}$ & $5.13 E+09 \mathrm{~Pa}$ \\
$G_{T N}$ & $3.6 \quad E+09 \mathrm{~Pa}$ \\
$\nu_{L T}=\nu_{L N}$ & 0.4542 \\
$\nu_{T N}$ & 0.4 \\
thickness & $203 E-06 \mathrm{~m}$ \\
density & $4060 \mathrm{~kg} / \mathrm{m}^{3}$ \\
$d_{e L L}$ & $3.09 E-10 \mathrm{~m} / \mathrm{V}$ \\
$d_{e L T}$ & $0 \mathrm{~m} / \mathrm{V}$ \\
$d_{e T T}$ & $-1.29 E-10 \mathrm{~m} / \mathrm{V}$ \\
$\mathrm{D} 3$ & $-1800 / 1.114 E-06 \mathrm{~V} / \mathrm{m}$ \\
\hline
\end{tabular}

The first case introduced above that is compared against the baseline blade is the zeroactuation case. This may represent a normal condition where no current has yet been passed to the blade, or it may also represent a malfunctioning electrode. The other two cases refer to the actuators running normally under a DC or AC current. The final blade structure will incorporate a feedback system with sensors that will measure the induced, azimuth-dependent loads. Thus the AC current is considered to be better suited for the actual application.

Table 5.3 displays the results of the stiffness values for the baseline, inactive and active scenarios. A DC current is represented here for the values shown in the table for force and moment contributions from actuation, $F_{x}^{(a)}, M_{x}^{(a)}, M_{y}^{(a)}$, and $M_{z}^{(a)}$. These values for active contributions are zero for the 'zero-actuation' case, when no current is sent to the piezoelectrics.

It should be noted that Design No. 1 contains active plies oriented $\pm 45^{\circ}$ with respect to the elastic axis for a given layer, signifying that opposing currents will be applied. That is, when one positively oriented layer is actuated with a negative voltage it will expand, whereas the negatively oriented layer that is positively charged will contract. 
Table 5.3: Stiffness values for Design 8

\begin{tabular}{|l|c|c|c|}
\hline & Baseline & 2 active plies & 4 active plies \\
\hline$K_{11}(\mathrm{~N})$ & 10391482.9 & 10279729.5 & 10075885.9 \\
$K_{12}(\mathrm{Nm})$ & 18743.8 & 12624.5 & 5085.6 \\
$K_{13}(\mathrm{Nm})$ & -371.2 & -605.0 & -805.6 \\
$K_{14}(\mathrm{Nm})$ & -24741.3 & -37514.2 & -48399.5 \\
$K_{22}\left(\mathrm{Nm}^{2}\right)$ & 316.2 & 268.9 & 196.6 \\
$K_{23}\left(\mathrm{Nm}^{2}\right)$ & 3.5 & 2.0 & 0.5 \\
$K_{24}\left(\mathrm{Nm}^{2}\right)$ & 184.2 & 101.8 & 25.2 \\
$K_{33}\left(\mathrm{Nm}^{2}\right)$ & 302.7 & 319.5 & 330.4 \\
$K_{34}\left(\mathrm{Nm}^{2}\right)$ & 142.7 & 144.9 & 146.9 \\
$K_{44}\left(\mathrm{Nm}^{2}\right)$ & 9566.2 & 9806.6 & 9993.9 \\
\hline$F_{x}^{(a)}$ & - & -312.7 & -631.8 \\
$M_{x}^{(a)}$ & - & -2.6 & -5.6 \\
$M_{y}^{(a)}$ & - & -0.1 & -0.1 \\
$M_{z}^{(a)}$ & - & -3.0 & -5.5 \\
\hline
\end{tabular}

In limiting the number of parameters for the scope of this thesis, two variables are considered in the ensuing parametric analysis. Blade pitch angle is varied from 0.0 to 0.3 rad in increments of $0.05 \mathrm{rad}$; the length of the blade over which actuators are embedded is also varied for $10,25,50$ and $95 \%$ of the blade span.

\subsection{Validation of Code}

Another validation study of the code that was written in MATLAB for stability results was performed against results of Hodges and Ormiston. Root loci for the first lead-lag, first flap, and torsion eigenvalues for a soft inplane rotor are obtained. The blade has uniform cross-sectional characteristics with no pretwist, $\theta=0$ rad, with parameters that are listed in Table 5.4 [41].

Elements of Figure 20(a) of Hodges and Ormiston were used to carry out the validation for comparing values of dimensionless frequencies. A low lead-lag nondimensional characteristic frequency value of $\omega_{v}=0.7$ signifies that the blade is soft; this example also 
Table 5.4: Parameters used for validation study

\begin{tabular}{||l|l||l|l||}
\hline$\omega_{v} / \Omega$ & 0.7 & $k_{m} / L$ & 0.025 \\
$\omega_{w} / \Omega$ & 1.15 & $k_{m_{1}} / k_{m_{2}}$ & 0.0 \\
$\omega_{\phi} / \Omega$ & $2.5,5.0,8.0$ & $\gamma$ & 5 \\
$\left(k_{A} / k_{m}\right)^{2}$ & 1.5 & $\sigma$ & 0.10 \\
$\theta$ & 0.0 & $c / L$ & $\pi / 40$ \\
$\beta_{p c}$ & 0.0 & $c_{d} / a$ & $0.01 / 2 \pi$ \\
$\Re$ & 0.0 & $b$ & 4 \\
\hline
\end{tabular}

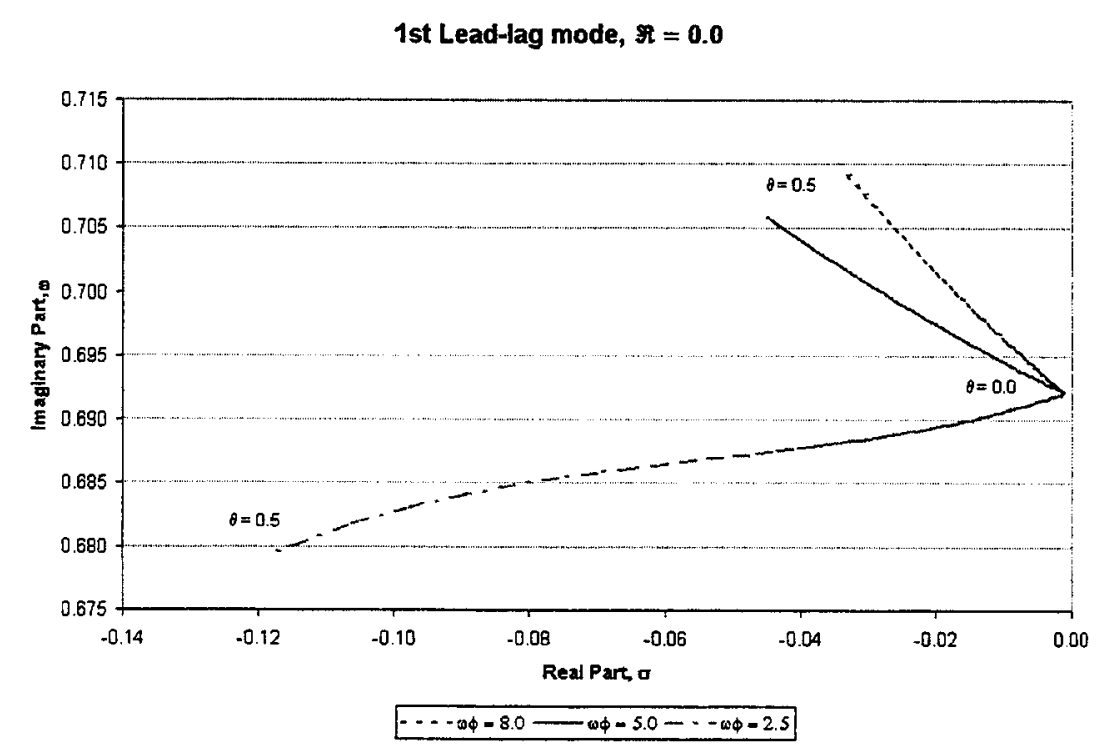

Figure 5.2: Locus of roots for 1st lead-lag mode: $\omega_{v}=0.7, \omega_{w}=1.15, \beta_{p c}=0.0$

assumes no coupling between the inboard and outboard segments $(\Re=0)$. Figures 5.2 to 5.5 show the locus of roots for the different modes for pitch angles ranging between $\theta=0.0$ and $\theta=0.5 \mathrm{rad}$.

Excellent agreement between the results given by Figures 5.2 to 5.5 and those obtained by Hodges and Ormiston indicates that the steady-state equilibrium deflections and perturbation equations have been carried out effectively throughout the code. Refer to Figure 5.6 for the results of Hodges and Ormiston. Further analysis may therefore be carried out on the final three blade designs. 


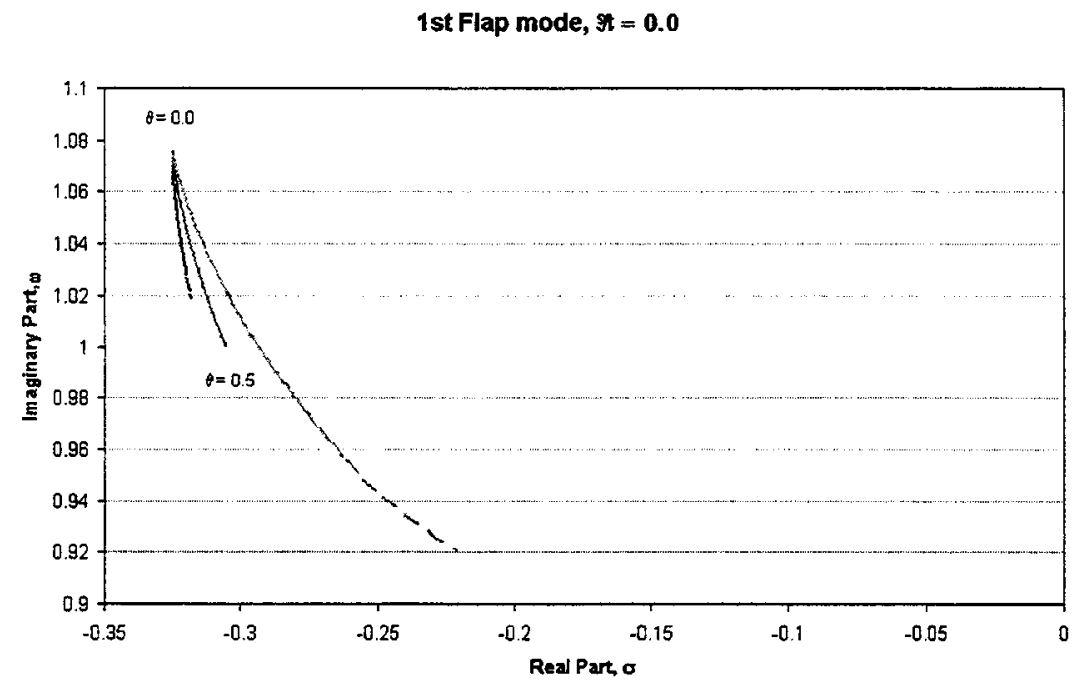

$-\cdots \infty \phi-8.0-\omega \phi-5.0-\cdots \infty \phi-2.5$

Figure 5.3: Locus of roots for 1st flap mode: $\omega_{v}=0.7, \omega_{w}=1.15, \beta_{p c}=0.0$

Torsion mode, $\Re=0.0$

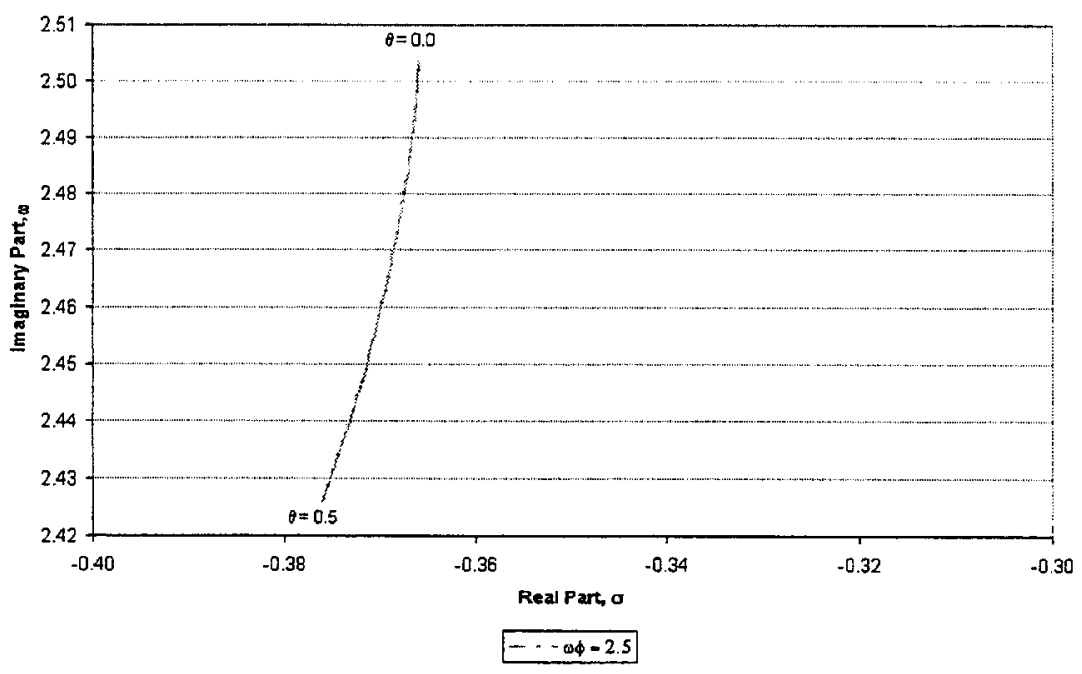

Figure 5.4: Locus of roots for torsion mode: $\omega_{v}=0.7, \omega_{w}=1.15, \beta_{p c}=0.0$ 


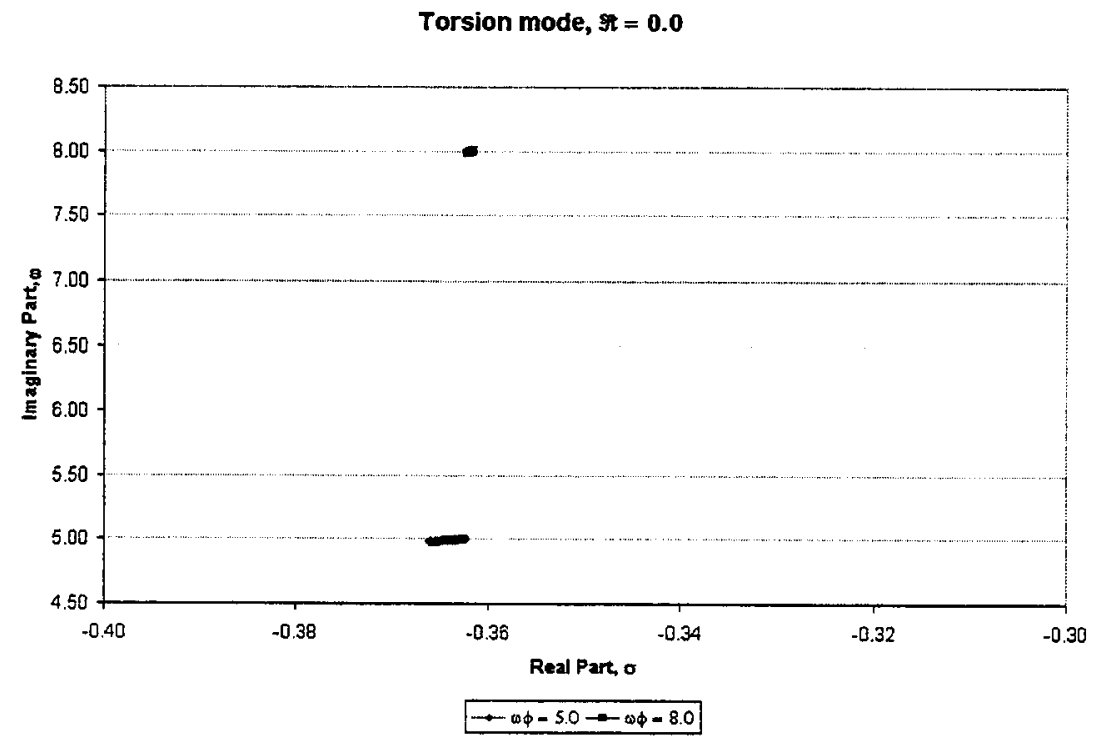

Figure 5.5: Locus of roots for torsion mode: $\omega_{v}=0.7, \omega_{w}=1.15, \beta_{p c}=0.0$

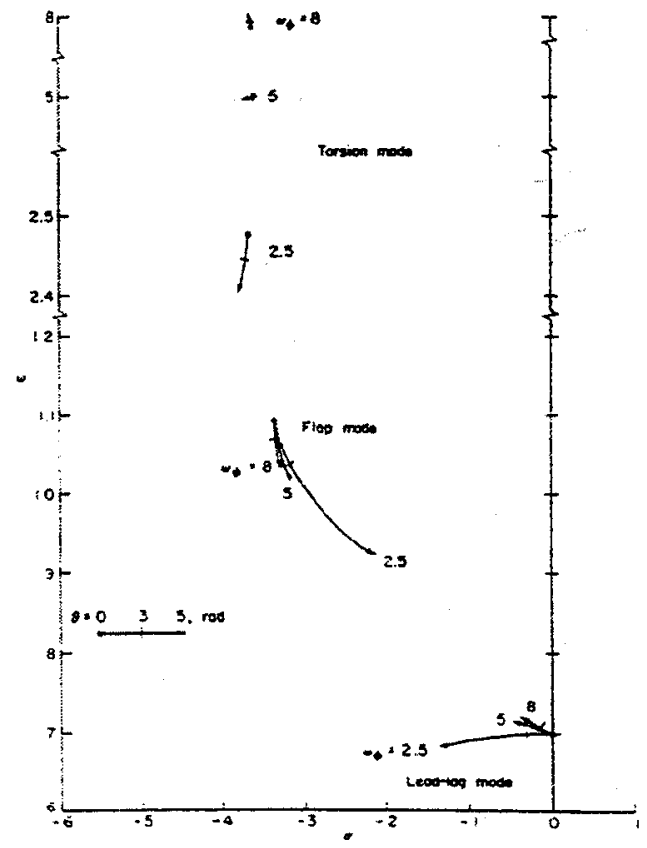

(a) $R=0.0$.

Figure 5.6: Locus of roots for a soft inplane rotor: Hodges and Ormiston 


\subsection{Steady-State or Equilibrium Deflections}

The nominal state at which the helicopter rotor blades are deflecting to without perturbation is known as the operating condition or the trim deflections. These deflections are given as $\bar{v}_{0}, \bar{w}_{0}$, and $\phi_{0}$ for the lead-lag, flap, and torsional directions, respectively. During hover, the total blade twist, for example, consists of the blade pitch angle, $\theta$, and the equilibrium torsional deflection $\phi_{0}$ :

$$
\phi_{T}=\theta+\phi_{0}
$$

compared to a perturbed state that is defined by:

$$
\phi_{T}=\theta+\phi_{0}+\delta \phi
$$

These equilibrium deflections arise from solving the $3 \mathrm{~N}$ nonlinear algebraic equations for the steady-state equilibrium constants, $V_{0 j}, W_{0 j}$, and $\Phi_{0 j}$, Eqs. (3.41) to (3.43).

Results for the equilibrium deflections at the tip for Design 8 are given here. The corresponding deflections for Design Cases 1 and 7 can be found in Appendix I. These dimensionless tip deflections are written in terms of the generalized coordinates as

$$
\begin{aligned}
\left.\bar{v}_{0}\right|_{t i p} & =2 \sum_{j=1}^{N} V_{0 j}(-1)^{j+1} \\
\left.\bar{w}_{0}\right|_{t i p} & =2 \sum_{j=1}^{N} W_{0 j}(-1)^{j+1} \\
\left.\phi_{0}\right|_{t i p} & =\sqrt{2} \sum_{j=1}^{N} \Phi_{0 j}(-1)^{j+1}
\end{aligned}
$$

Figures 5.7 through 5.9 show these trim tip deflections in the lead-lag, flap, and torsional directions for varying pitch angles and number of layers actuated. Recall that the 


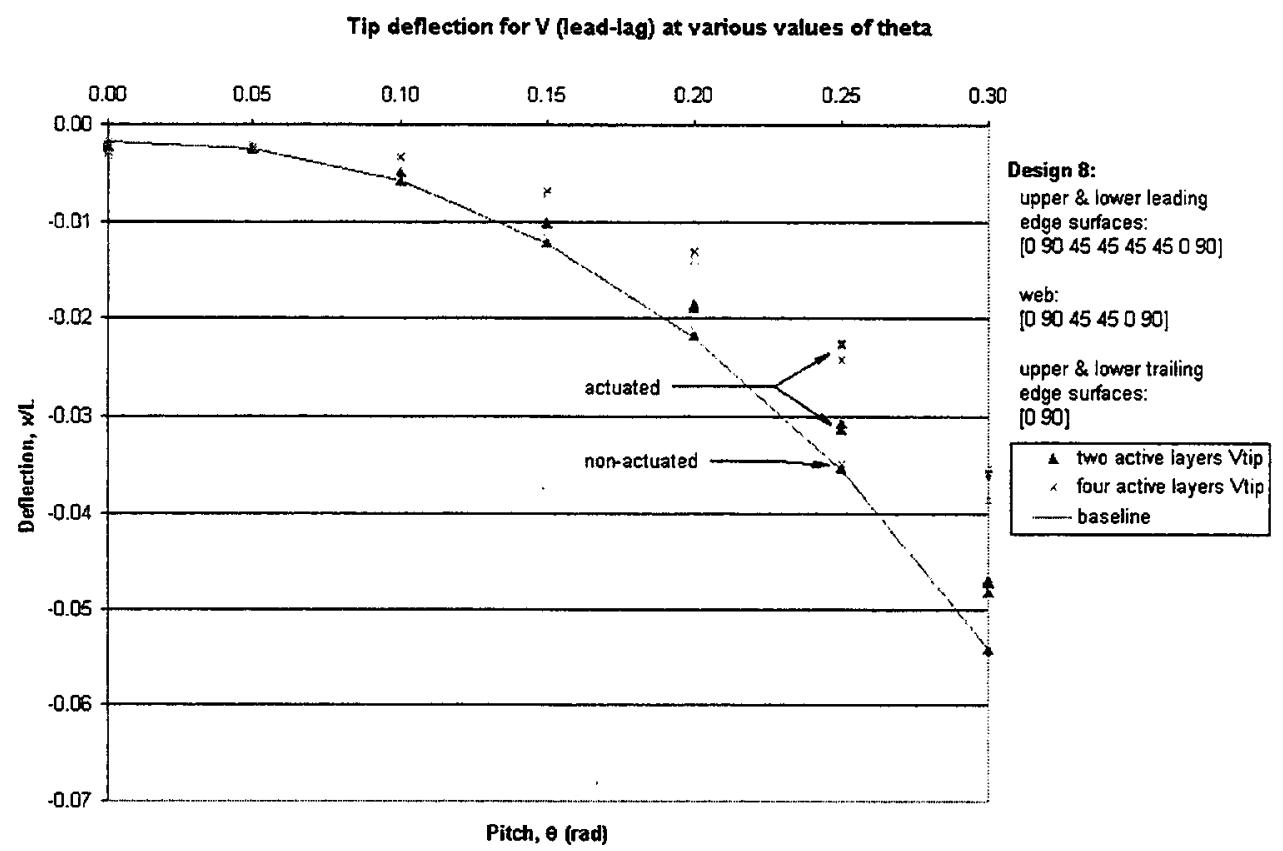

Figure 5.7: Equilibrium tip deflections in lead-lag bending

coupling factor, $\Re$, between the inboard and outboard segments has been set to one for the three designs. The baseline case is shown as a solid line, whereas the smart blade (both actuated and non-actuated) is represented by a series of data points. The data point closest to the baseline value represents the zero-actuation case, where no current is sent to the piezoelectrics. These values lie very close to those of the baseline blade except for high values of pitch $(\theta \simeq 0.3 \mathrm{rad})$. The cluster of points further away from the baseline and zero-actuation values represent the values for a blade whose length contains 10,25 , 50 and $95 \%$ AFC coverage. In general, as pitch angle is increased, thereby increasing the aerodynamic forces acting on the blade, magnitudes of deflection increase as well.

Comparison between the three design cases in lead-lag bending reveals that in all cases, the greater the number of active plies the greater the overall decrease in the magnitudes of deflection. Also the greater the percentage of spanwise blade coverage of AFCs, the less the resulting deflection. Design 7 experiences the most deflection, for four actuated layers 


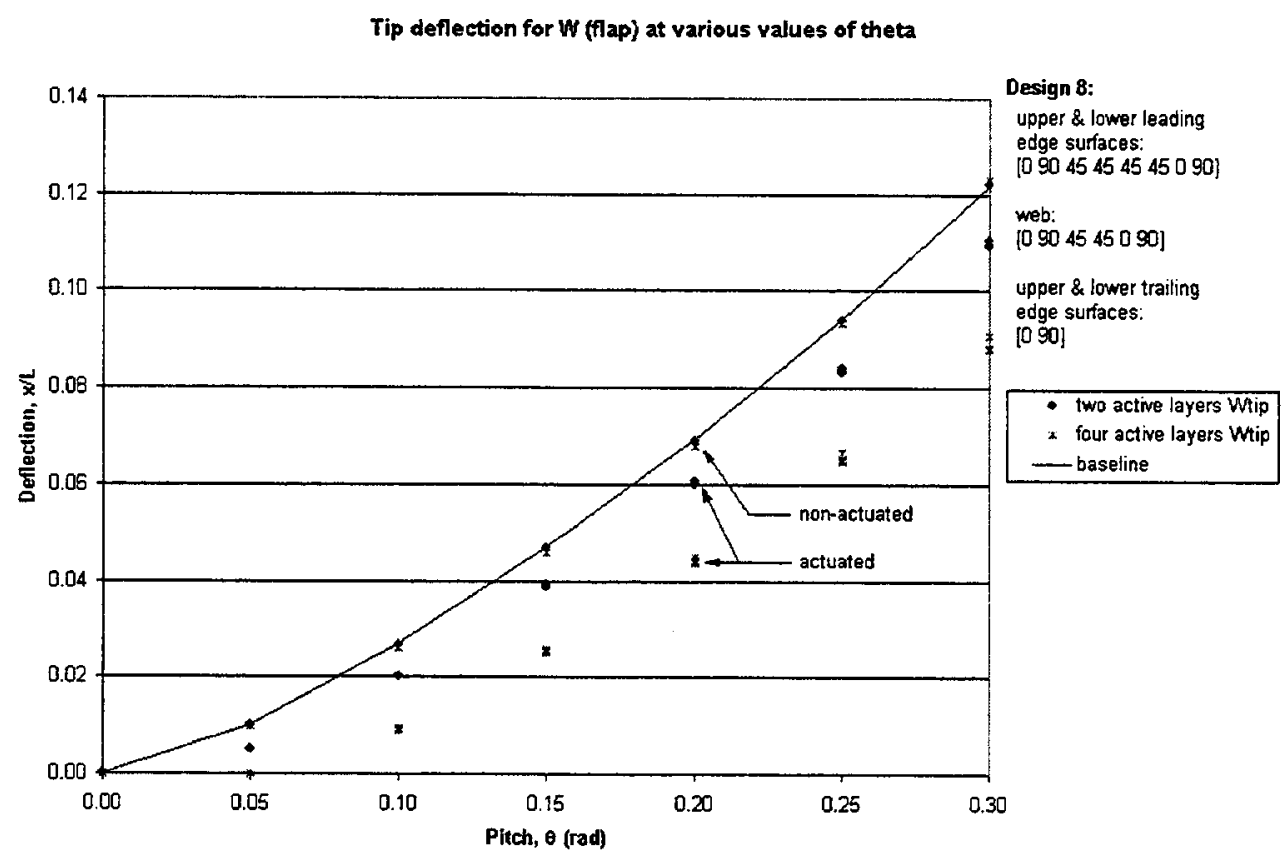

Figure 5.8: Equilibrium tip deflections in the flap bending

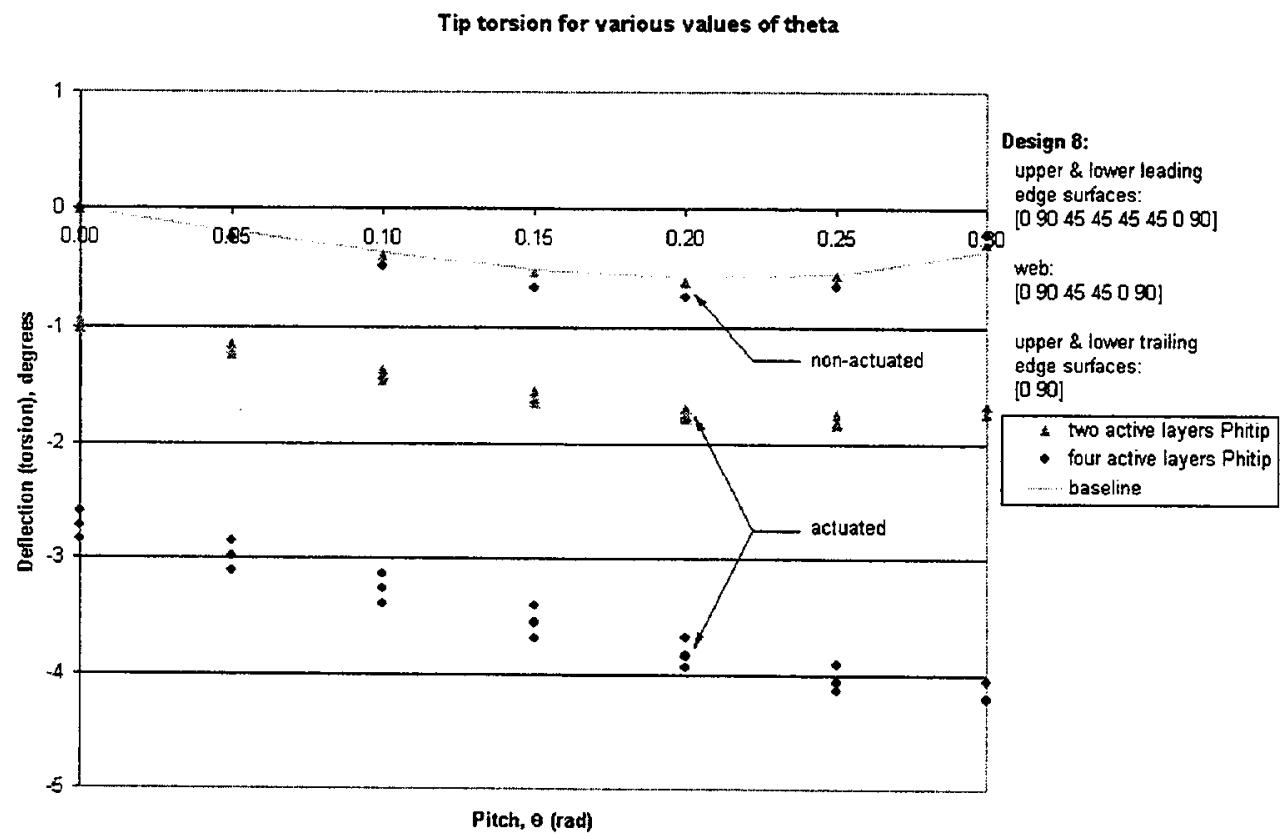

Figure 5.9: Equilibrium tip torsion 
Table 5.5: Torsion experienced at tip by smart blade designs

\begin{tabular}{|r|l|c|lc|c|}
\hline Design & Case & Twist & Pitch angle & Condition \\
\hline 1 & $\mathrm{a}$ & $-1.9^{\circ}$ & $\theta=0.21 \mathrm{rad}$ & Maximum twist \\
& $\mathrm{b}$ & $-3.5^{\circ}$ & $\theta=0.25 \mathrm{rad}$ & " \\
& $\mathrm{a}$ & $-1.2^{\circ}$ & $\theta=0.0 \mathrm{rad}$ & Twist at zero pitch \\
\hline & $\mathrm{b}$ & $-2.3^{\circ}$ & $\theta=0.0 \mathrm{rad}$ & " \\
\hline 7 & $\mathrm{a}$ & $-1.8^{\circ}$ & $\theta=0.25 \mathrm{rad}$ & Maximum twist \\
& $\mathrm{b}$ & $-3.3^{\circ}$ & $\theta=0.3 \mathrm{rad}$ & $"$ \\
& $\mathrm{a}$ & $-0.9^{\circ}$ & $\theta=0.0 \mathrm{rad}$ & Twist at zero pitch \\
& $\mathrm{b}$ & $-2.0^{\circ}$ & $\theta=0.0 \mathrm{rad}$ & " \\
\hline 8 & $\mathrm{a}$ & $-1.8^{\circ}$ & $\theta=0.25 \mathrm{rad}$ & Maximum twist \\
& $\mathrm{b}$ & $-4.2^{\circ}$ & $\theta=0.3 \mathrm{rad}$ & " \\
& $\mathrm{a}$ & $-1.0^{\circ}$ & $\theta=0.0 \mathrm{rad}$ & Twist at zero pitch \\
& $\mathrm{b}$ & $-2.7^{\circ}$ & $\theta=0.0 \mathrm{rad}$ & " \\
\hline
\end{tabular}

in the blade section, whereas Design 8 experiences the least. This is generally the same for only two active layers present in the cross-section, although the difference in magnitudes of deflection is less noticeable.

Bending in the flapping sense also reveals that lower magnitudes of deflection are experienced in a blade for a greater number of actuated plies. Here also, Design 8 experiences the least deflection and Design 7 the most with four active plies present. Cases with two active plies show little change in the corresponding values between the three designs.

The final comparison for tip deflections is in twist. Values of torsional deflection are shown in degrees rather than radians to better appreciate the magnitudes. The opposite effect for degree of deflection between designs is seen here. Four active layers embedded in the smart blade results in a maximum of $-4.2^{\circ}$ of twist for Design 8 , compared to $-3.3^{\circ}$ for Design 7. When comparing these values to the three baseline blades, which only achieve between $-0.6^{\circ}$ to $-0.8^{\circ}$ of twist, the effect that the AFCs produce is clearly seen. Table 5.5 summarizes some of the values for twist in all three designs. 


\subsubsection{Actuation over Blade Span}

Analyses were also carried out to relate percent of blade actuated over the total span for various degrees of pitch. This took into account how much bending or torsion deflection was achieved depending on what percent of the blade was embedded with actuators. Figures 5.10 through 5.15 show twist deflection levels for Design 8a; lead-lag and flap results for this design case are found in Appendix J. These plots give a good indication of what length of the blade should be embedded with actuators when applied with an electric field. Bailey describes the relationship between torsional strain, GJ, and rate of change of twist, $\phi^{\prime}$, as a function of blade span. The highest strain levels are shown to be at the root, indicating that this is the optimal location for the actuators. Although deflection levels for both lead-lag and flap bending are of the same order of magnitude in actuating $10 \%$ of the blade as with $95 \%$ of the blade, the torsion graphs of Figures 5.12 to 5.15 indicate that the magnitude of torsion is not constant over the blade span for the actuated blade. The portion of the blade located after the segmented length of actuators shows minor fluctuations in the rate of change of $\phi$. This could be due to a numerical approximation.

Designs 1 and 7 show the same type of behaviour for bending and twist as a function of span-wise actuation, and so results have not been included. Since the rate of change of $\phi$ reduces for the region of the blade located after the actuators, the effectiveness of the achieving uniformity in twist is also reduced. Due to this nonuniformity in twist for small lengths of blade actuation, piezoelectrics should be placed along the full blade length to achieve the best results.

These equilibrium deflections $V_{0 j}, W_{0 j}$, and $\Phi_{0 j}$ will now be used as the coefficients of the $3 \mathrm{~N}$ perturbation equations to solve the homogeneous equations of motion. 


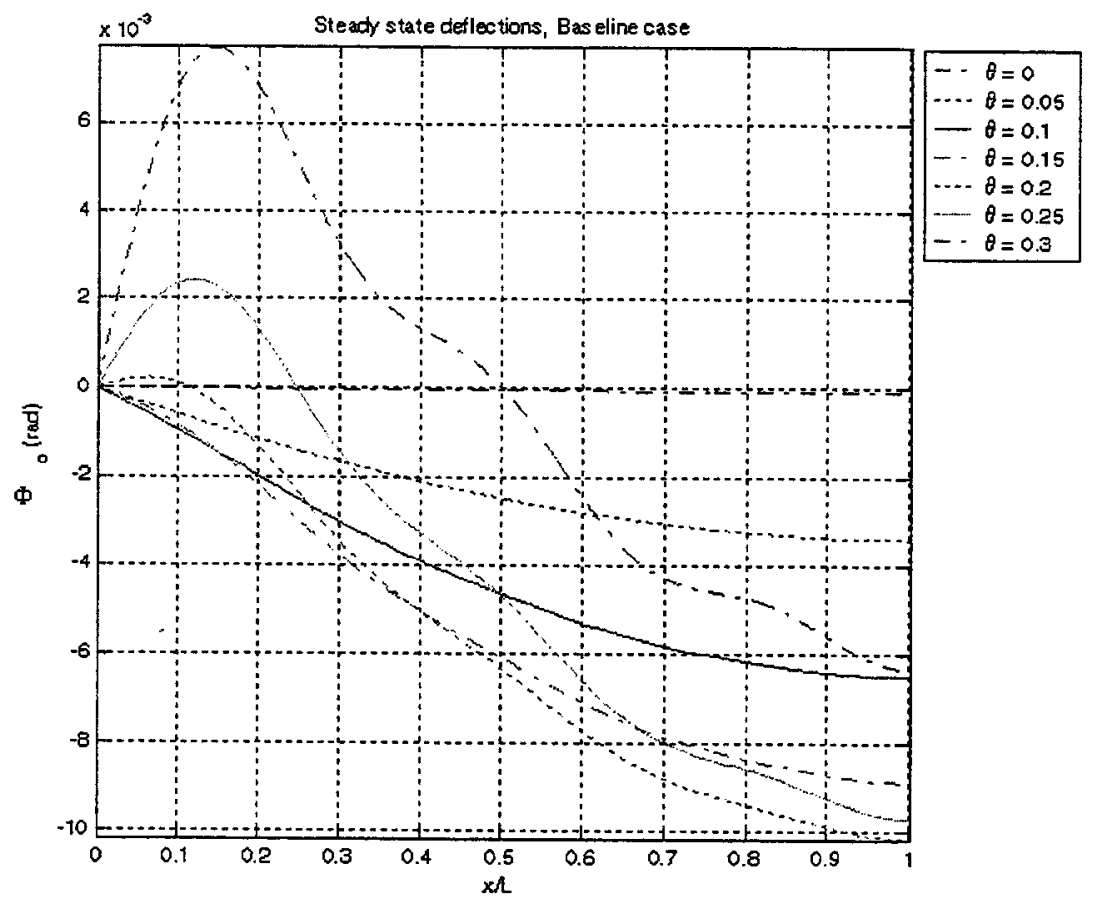

Figure 5.10: Torsional levels for the baseline case of Design 8

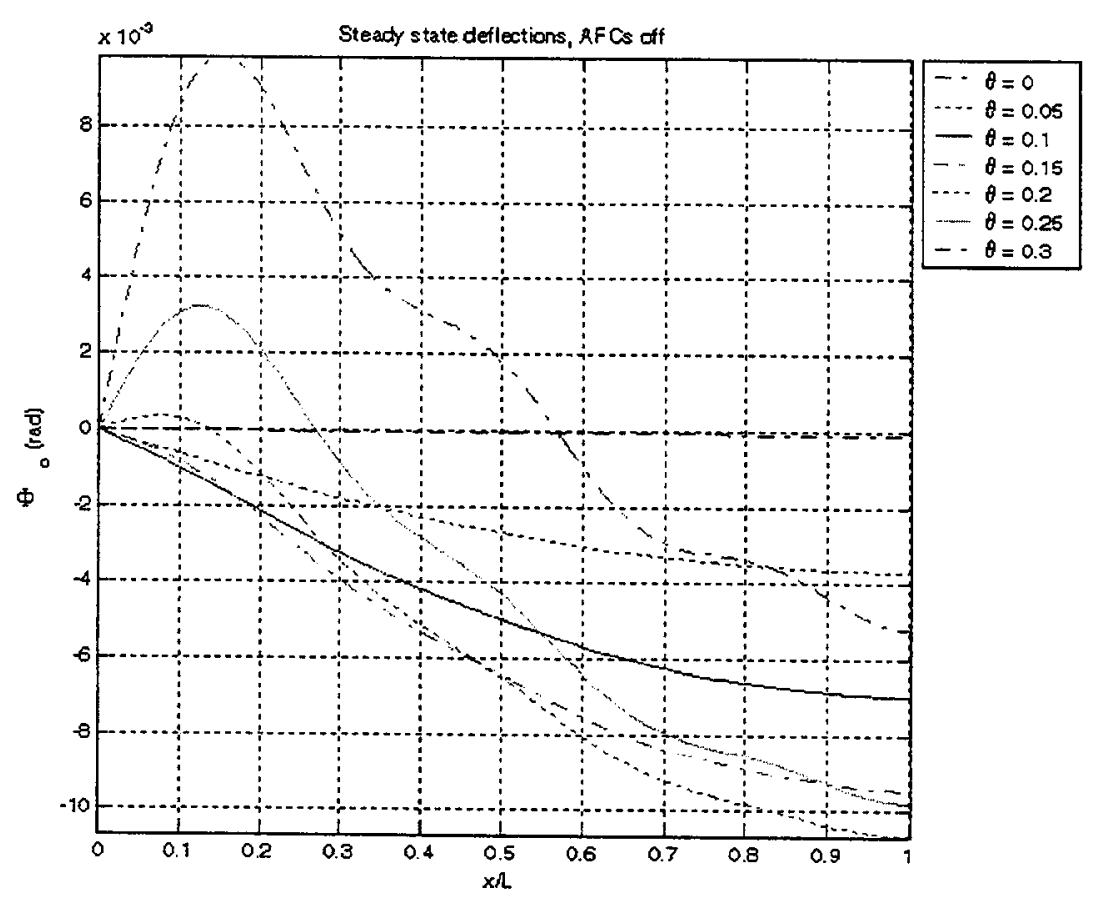

Figure 5.11: Torsional levels for zero applied load in Design 8 


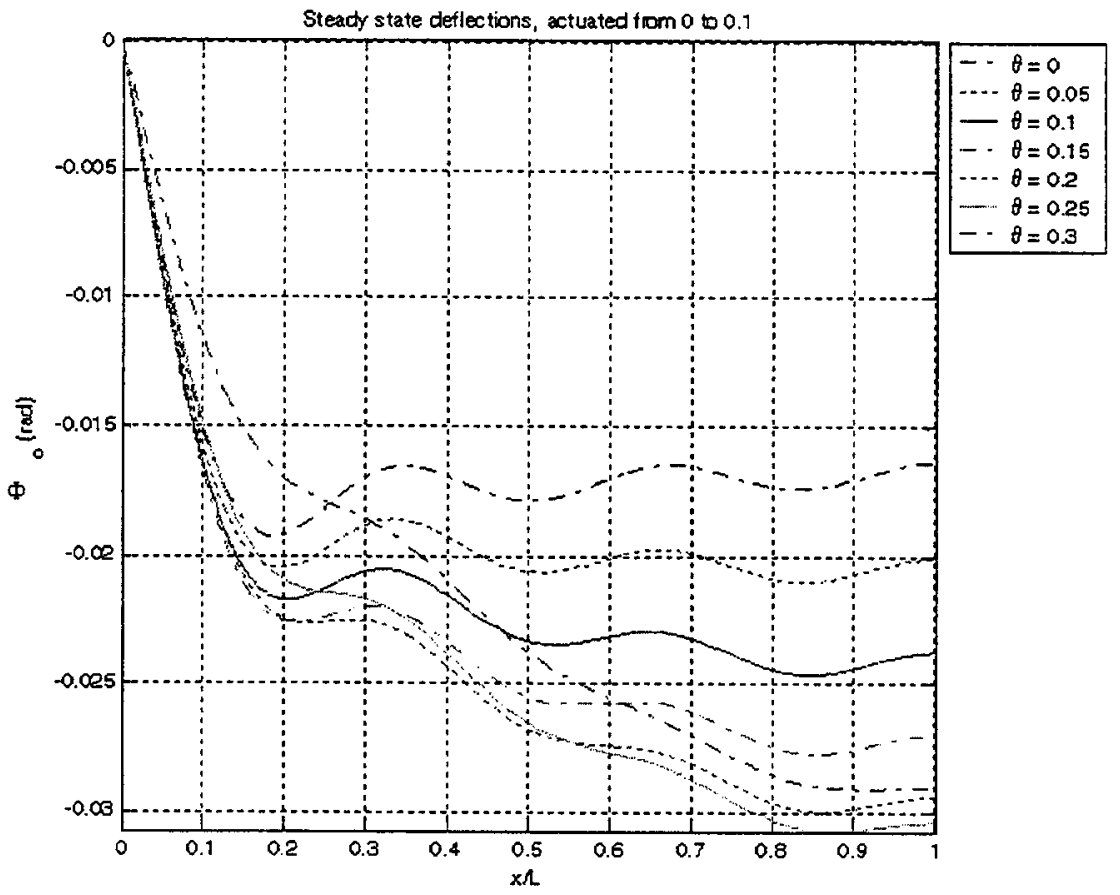

Figure 5.12: Torsional levels for $10 \%$ span-wise actuation in Design 8

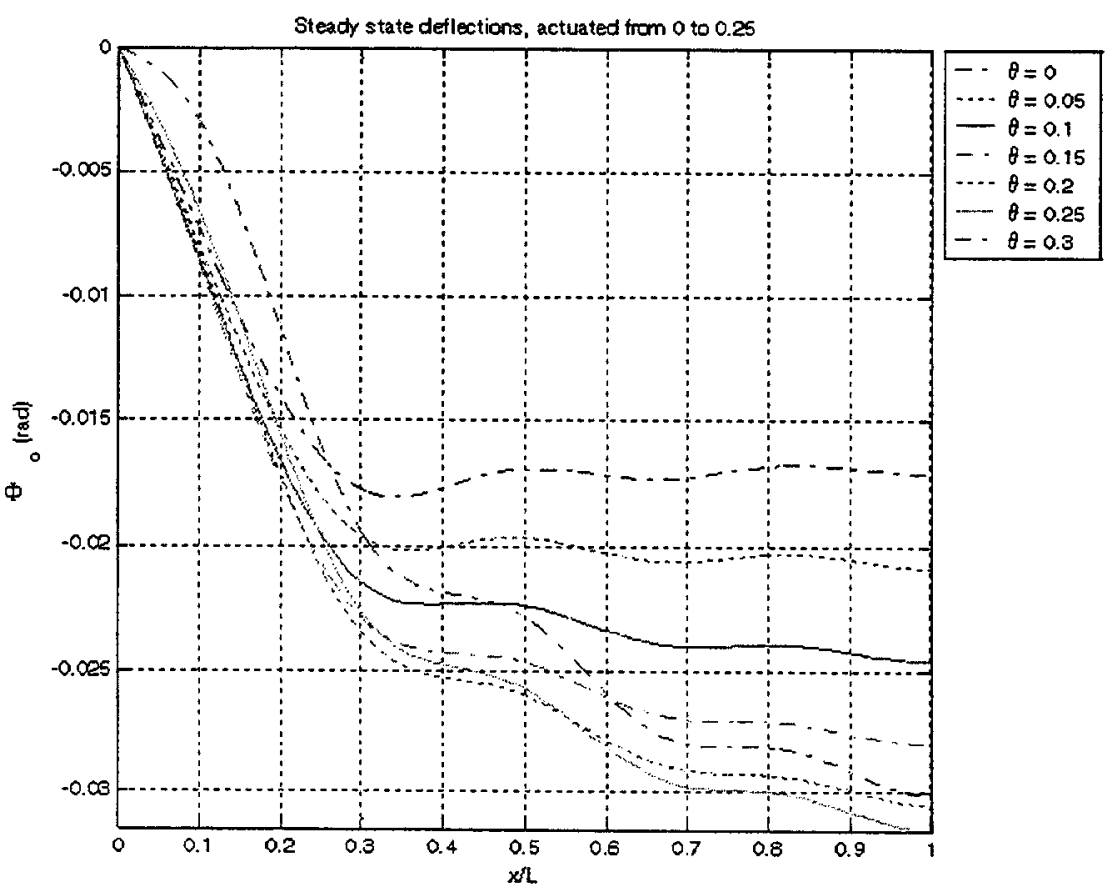

Figure 5.13: Torsional levels for $25 \%$ span-wise actuation in Design 8 


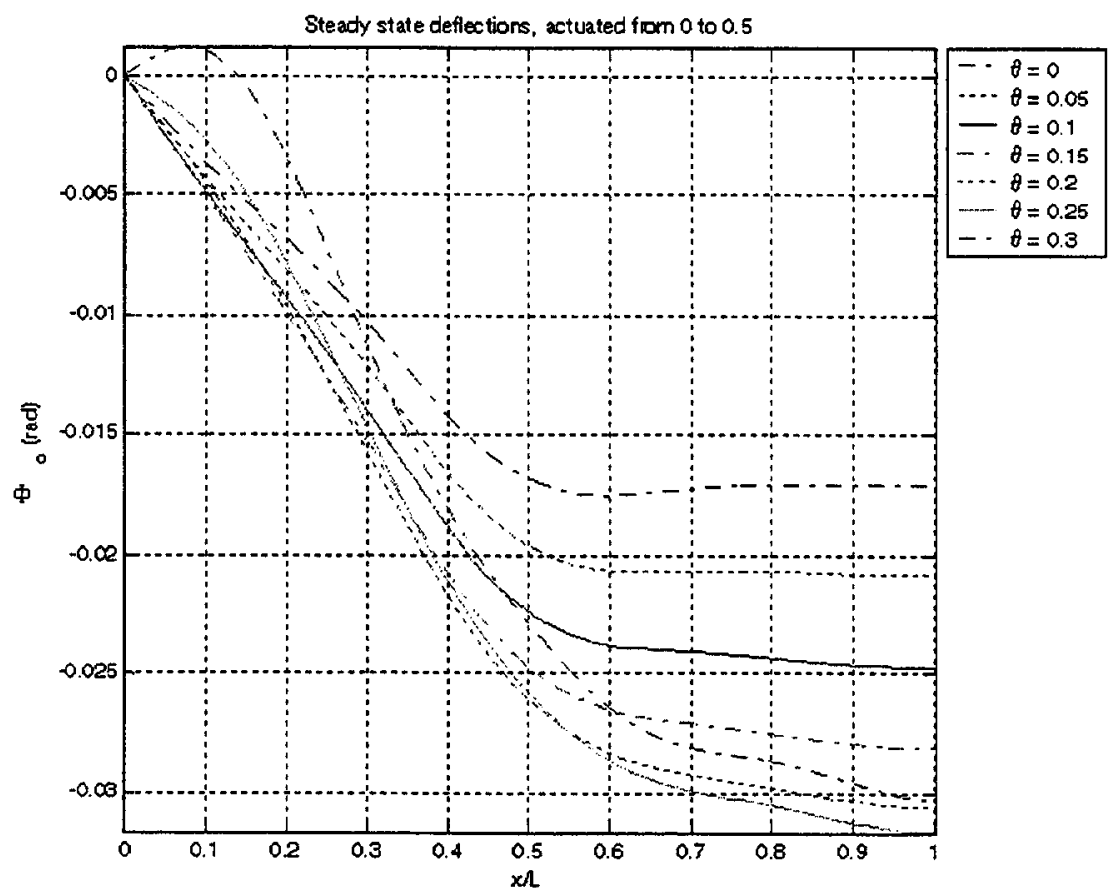

Figure 5.14: Torsional levels for $50 \%$ span-wise actuation in Design 8

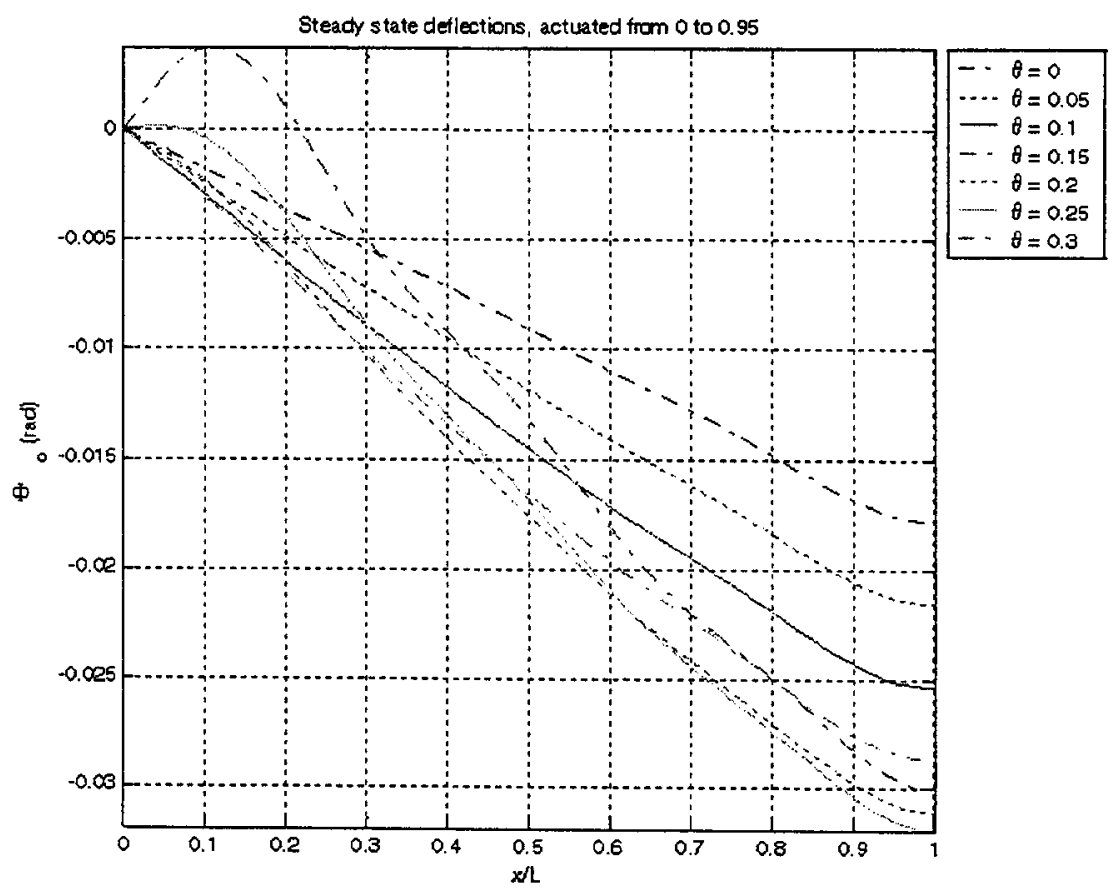

Figure 5.15: Torsional levels for $95 \%$ span-wise actuation in Design 8 


\section{$5.4 \quad$ Stability}

The stability of a rotor blade is demonstrated in two manners. The eigenvalues or natural frequencies of the homogeneous equation may show stable values or indications of divergence or flutter.

\subsubsection{Natural Frequencies}

There are two types of instabilities that may occur in a helicopter rotor blade - they are divergence and flutter. Both depend on the structural properties of the lifting surface (stiffness, mass) and flight conditions (airspeed, altitude, Mach number). In either case, if the excitation to the structure is removed the system's response will not attenuate. The only way of achieving stability is by changing the above structural and aerodynamic operators. Conversely, the response-stability problem (e.g. BVI - blade vortex interactions) is directly related to the external perturbations (which may be represented by $u$ ). All instabilities are regarded as dangerous to the system as structural failure may occur instantaneously, and so recognition is vital in design. The key identification parameter for instability lies in positive damping values.

Stability analyses were carried out on all design cases, but in consideration of high twist levels and good stability results, only Design Case $8 \mathrm{a}$ will be discussed and later analysed for response behaviour. All designs, however, exhibit good levels of stability. The excitation modes that will be examined are the 1st lead-lag, the 1st and 2nd flap, and the torsion modes. The smart blade is actuated with both an $1800 \mathrm{~V}$ magnitude $\mathrm{DC}$ and $\mathrm{AC}$ current and results are compared against the baseline and zero-actuation cases. The solution to the stability problem requires the solving of the homogeneous equation, Eq. (3.44) in order to obtain the eigenvalues.

Applying an $\mathrm{AC}$ current to the blade signifies that the homogeneous equation is not only 


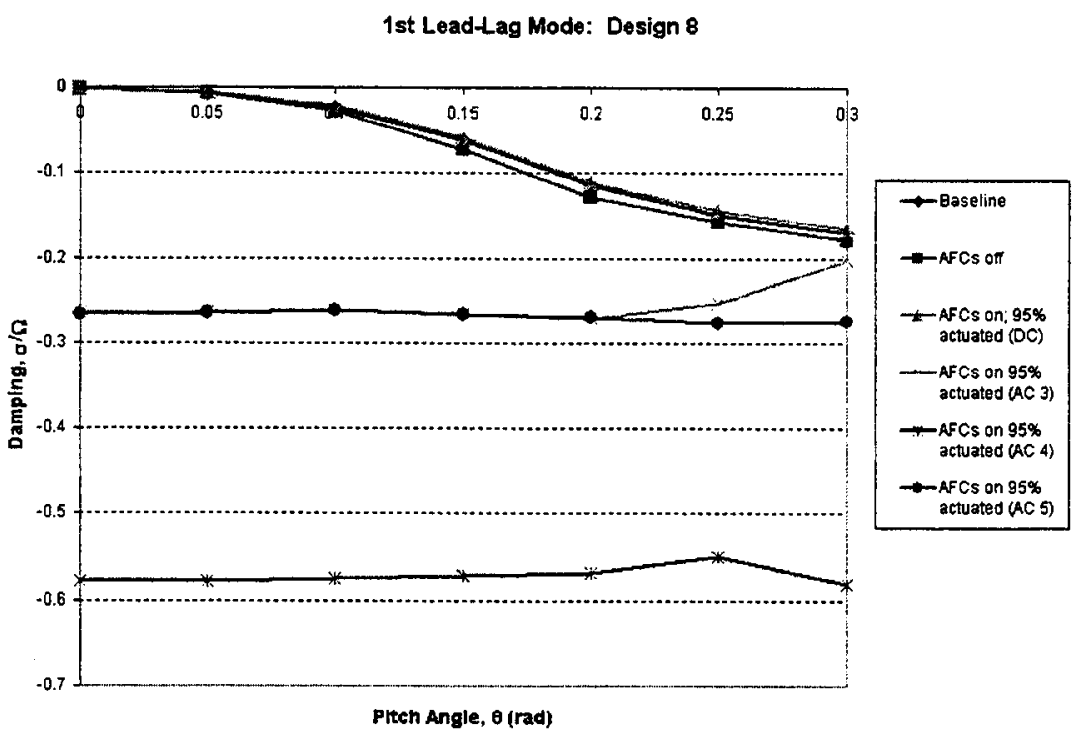

Figure 5.16: Damping of the 1st lead-lag mode for Design 8a

a function of position, $x$, but that it is also a function of time. This enables a manipulation of the blade as it experiences different loads throughout its rotation as in Figure 5.1. The voltage applied to the electrodes is defined by a sine curve whose magnitude is

$$
V= \pm \frac{1800}{1.114 * 10^{-6}} \sin \left(\frac{\omega t}{\Omega}\right)
$$

where $\omega$ is the frequency of time actuation and $\Omega$ is the rotor's hover speed. For an $\mathrm{N}$ bladed helicopter rotor $\mathrm{N}-1, \mathrm{~N}$ and $\mathrm{N}+1$ frequencies should be applied for one complete rotation of the blade. The Floquet method may then be used to solve for the eigenvalues $[14,59,60]$.

\section{1st Lead-Lag Mode}

This mode, as with the three other modes studied, shows no tendencies towards divergence or flutter. Figures 5.16 and 5.17 illustrate this mode. However, the baseline case, the DC case, and the zero-actuated case show weak damping at low pitch values. It should be noted 


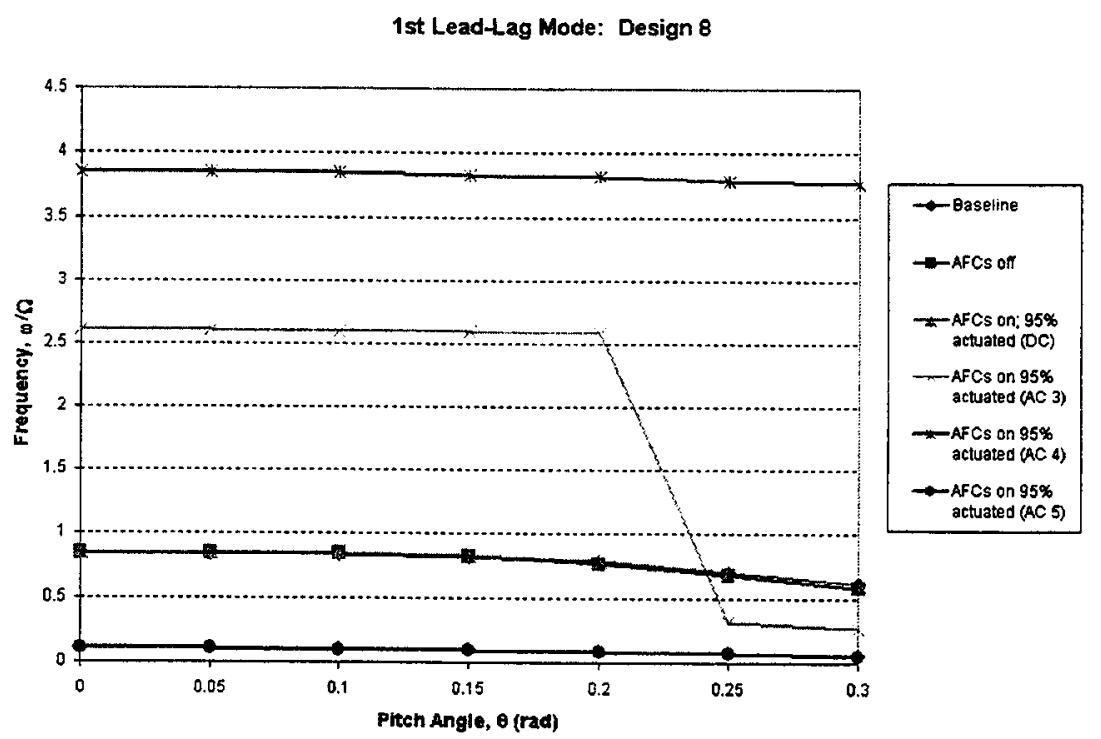

Figure 5.17: Frequency of the 1st lead-lag mode for Design 8a

that in all modes the blade that is undergoing a DC current exhibits similar behaviour to the baseline and zero-actuation blades. The AC cases show much more damping than the first three cases up until approximately 0.25 rad of pitch. AC3, AC4, and AC5 correspond to $\mathrm{N}-1, \mathrm{~N}$, and $\mathrm{N}+1$ times the frequency of rotation for this 4-bladed rotor. As stated earlier in the chapter, a divergence instability is characterized by frequencies equalling zero. However as this mode has no positive damping values, as well as no zero-valued frequencies, divergence is not a concern and this mode is stable.

\section{1st Flap Mode}

Figures 5.18 and 5.19 display the results for the 1st flap mode. The 1st flap mode shows good damping levels for all cases except AC5; this load condition for low pitch angles has weak damping bordering the positive Laplace domain. There may also be indications of the $\mathrm{AC} 4$ blade tending towards a flutter instability, however this would have to be verified at higher pitch angles. AC3 closely follows the trends of the baseline, zero-actuation, 


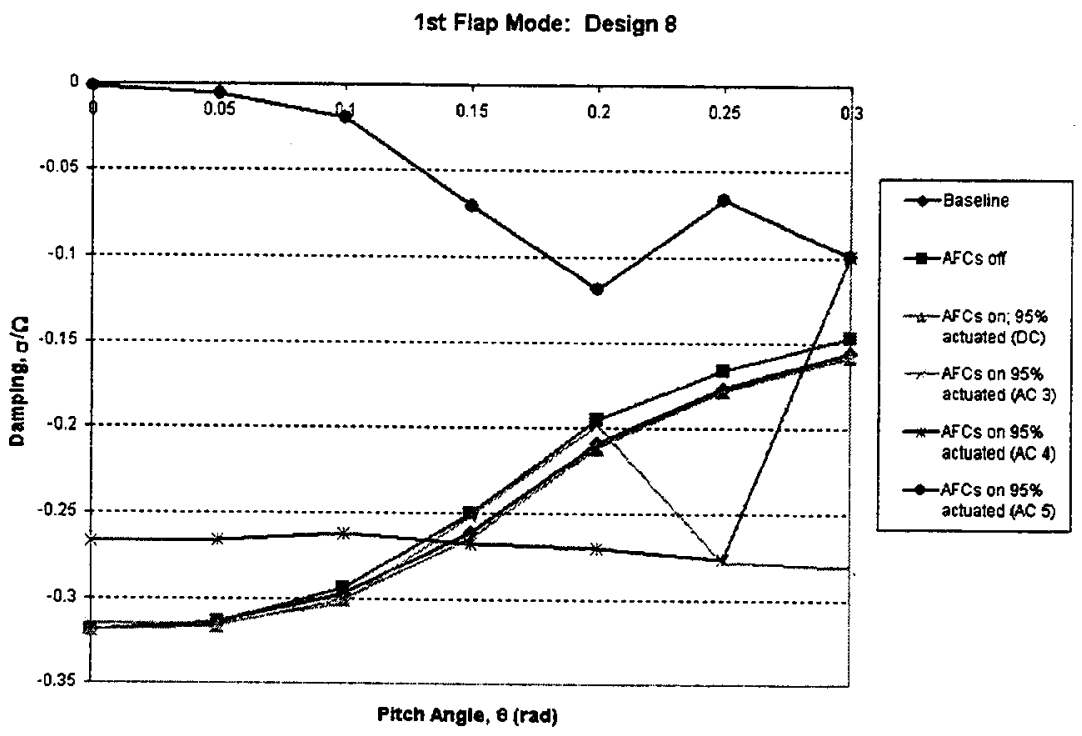

Figure 5.18: Damping for the 1st flap mode for Design 8a

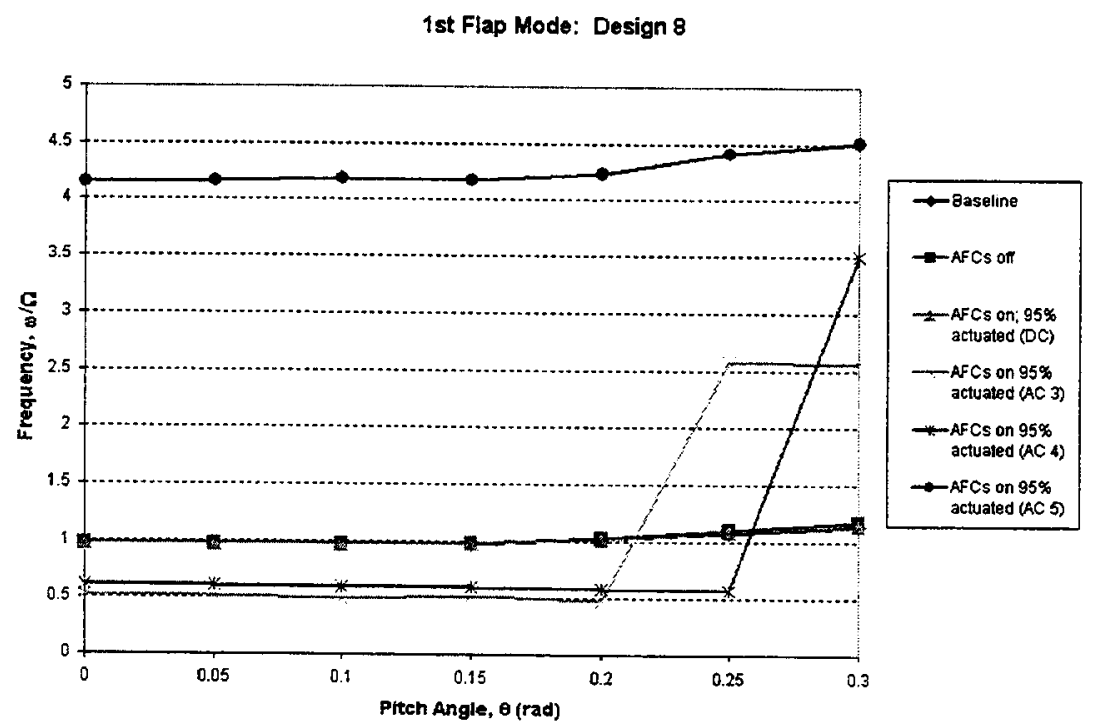

Figure 5.19: Frequency for the 1st flap mode for Design 8a 
and DC cases. Given that this mode has no positive damping values for the pitch angles studied, it may be considered stable.

\section{2nd Flap Mode}

This mode shows good damping levels for all actuated and non-actuated cases except for AC4, whose damping improves with the level of pitch. AC3 shows the highest degree of damping until pitch angles of approximately $0.25 \mathrm{rad}$ where the possibility exists for it to be approaching the right hand side of the Laplace domain. However for the region shown here, this mode is stable, having no indications of divergence or flutter. Refer to Figures 5.20 and 5.21 for these results.

\section{Torsion Mode}

It is worth noting that for this mode, the blade which is actuated at 3 times the frequency of the rotor frequency, AC3, shows weak damping levels. This may be compared with AC4 for the 2nd flap mode, AC5 for the 1st flap mode, and the DC blade in the 1st lead-lag mode, indicating that each actuation case corresponds to weak damping levels for one certain mode. The remaining actuated and non-actuated cases for the torsion mode show good levels of damping with AC4 nominally exhibiting weaker levels of damping as seen in Figures 5.22 and 5.23. This mode, as with the previous modes studied, has no divergence or flutter and is therefore considered stable. 


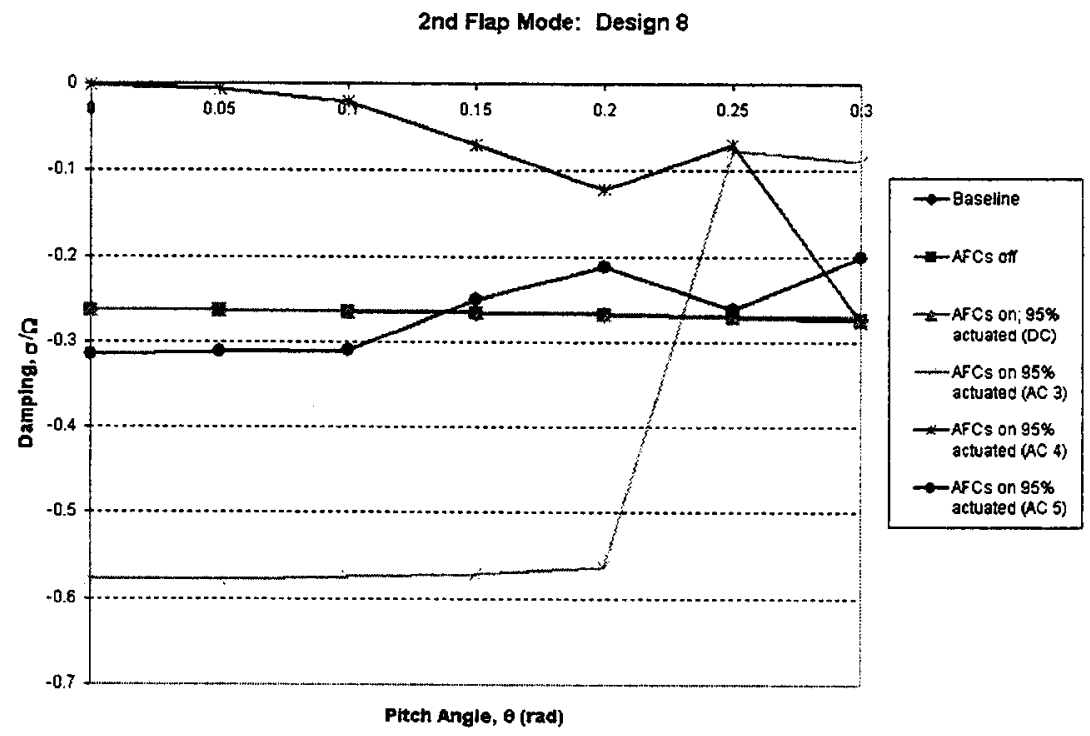

Figure 5.20: Damping for the 2nd flap mode for Design $8 \mathrm{a}$

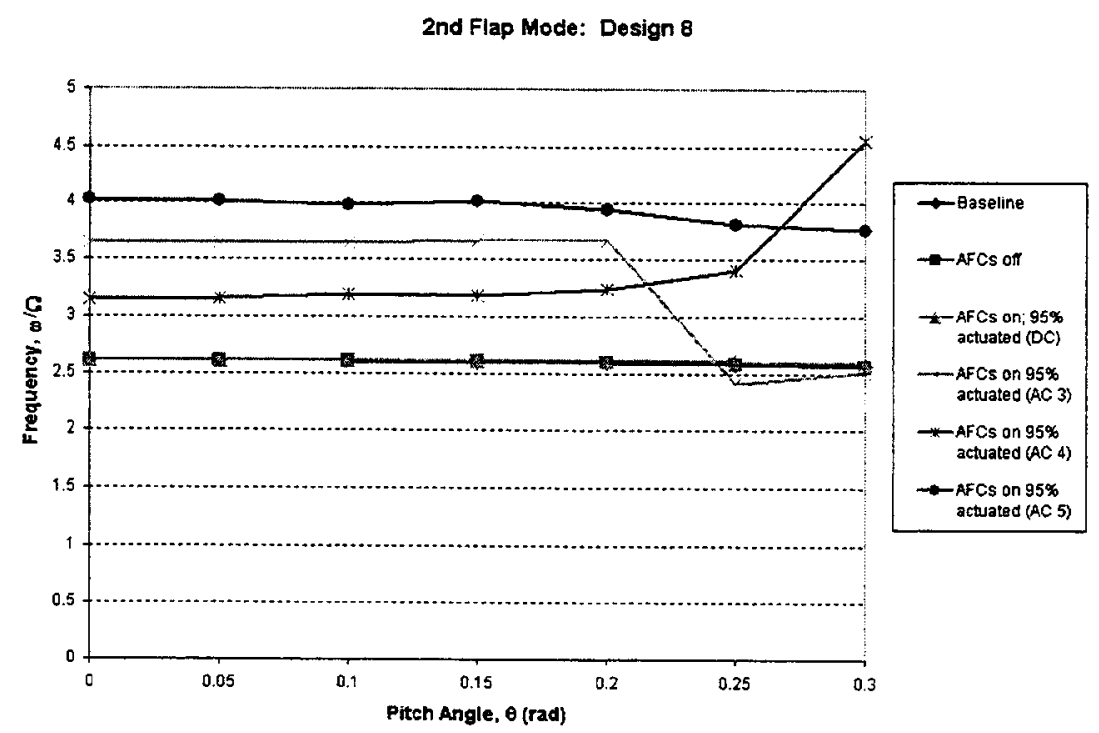

Figure 5.21: Frequency for the 2nd flap mode for Design 8a 


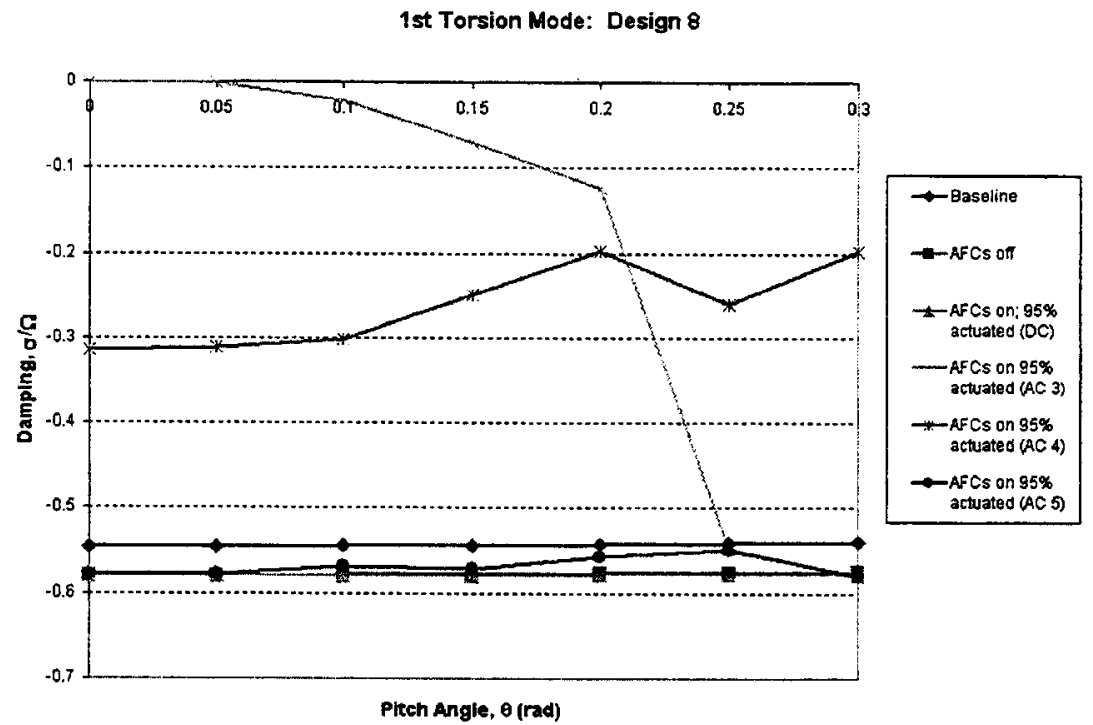

Figure 5.22: Damping for the 1st torsion mode for Design 8a

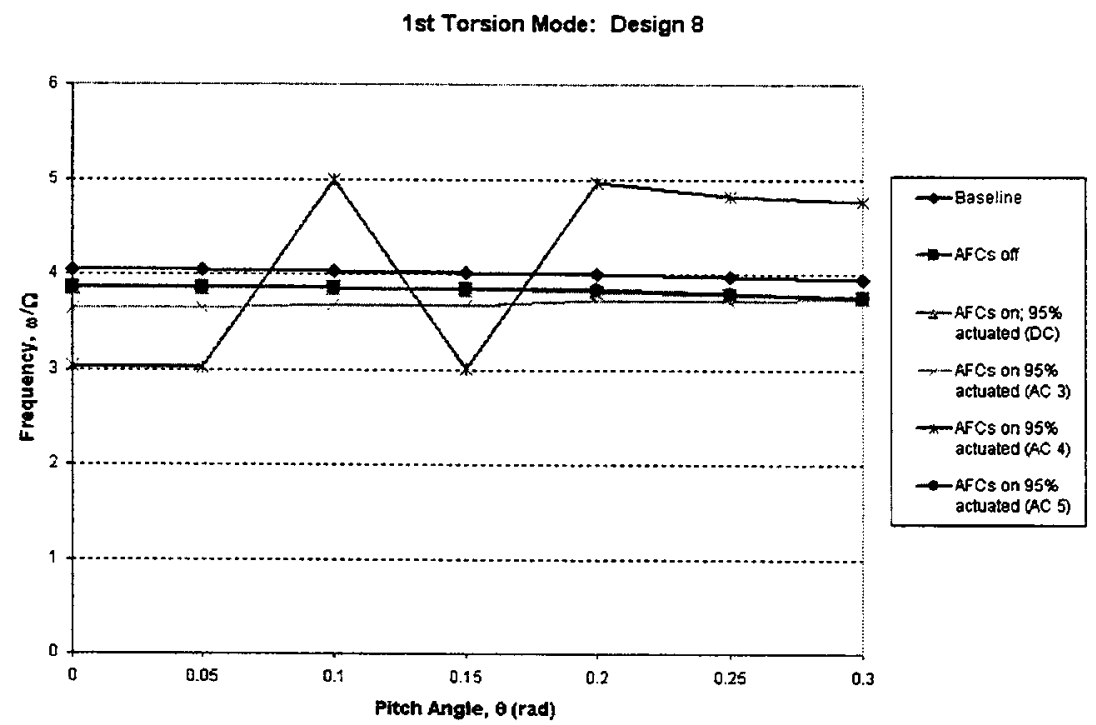

Figure 5.23: Frequency for the 1st torsion mode for Design 8a 


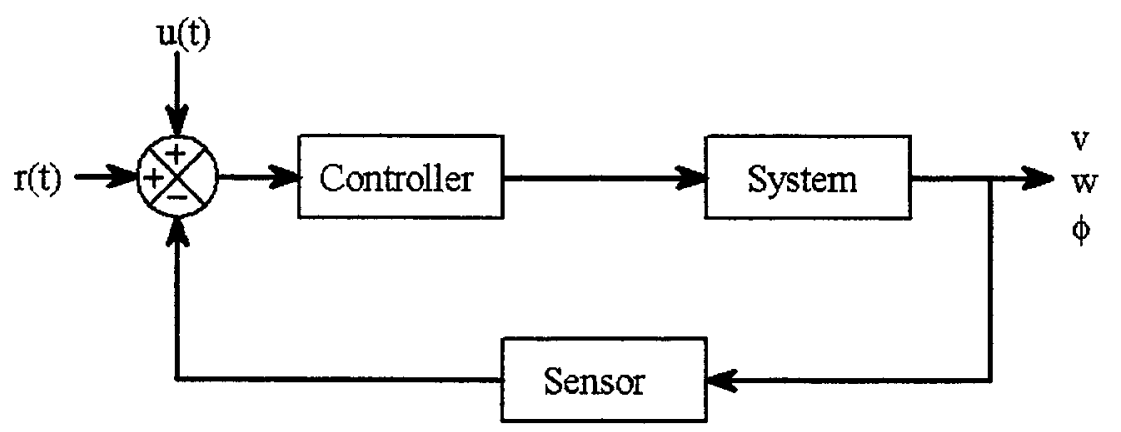

Figure 5.24: Block diagram of the smart rotor blade

\subsubsection{Response to Impulse}

It is envisaged that a smart blade containing AFCs will be described in its complete form as having full sensing and actuation capabilities. It's operation may therefore be described by the closed-loop feedback system shown in Figure 5.24. With a control system established, a transfer function will enable proper implementation of the control systems to be used for future studies. The Sensing device, usually another form of piezoelectric, measures the time-varying output $v, w$, and $\phi$. The input for this rotor blade application are the various perturbations that are experienced by the blade throughout its rotation. These perturbations, which are shown in Figure 5.1 are the periodic forces at the root of the vibration problem. Encompassed within the Controller is the forcing function $\{F\}$ providing the actuation authority to dampen or cancel the induced vibrations. The distributed AFCs along the blade span are the 'meat' of the Controller. Along with the twisting motion produced by the AFCs, when a current in AC form is sent throughout the piezoelectrics, the resulting actuation is able to directly address the periodic disturbances. The final component is the System which defines the behaviour through the equations of motion that determine the output for $v, w$, and $\phi$.

Along with the excitations outlined in Figure 5.1, to which the smart blade is responding periodically, the blade is also subject to impulsive gusts and landing forces. The most 
common effect on the rotor structure due to these impulses is BVI, which can also be handled by actuation of the smart blade. BVI is characterized as a 'response' problem, because unlike a stability problem, if these perturbations or excitations are removed, the vibrations will cease with time. This is because the system is naturally damped by either aerodynamic loads or inherent structural damping.

Buffeting (the sister to BVI) in airplanes is commonly found at the tail and is usually a result of turbulent flow from the main wings. A sudden gust on the main lifting surfaces will cause a sudden increase in the angle of attack, leading to flow separation and thus turbulent flow to the tail [61]. While solutions exist for airplanes - e.g. locating the tail outside of the region of disturbance - in helicopters the problem is slightly more complex. For an N-bladed helicopter each blade imparts unsteady flow to its counterpart. Thus, a combination of applying an AC current to the embedded piezoelectrics with the feedback system could provide a direct solution.

This thesis only introduces the control scheme that could be used in the blade system much is left to be described in future work. To begin modelling this system for Design $8 \mathrm{a}$ at a pitch angle of $\theta=0.05 \mathrm{rad}$, Eq. (3.44) is once again invoked, and the perturbations, $u(t)$, are taken into consideration:

$$
[M]\{\ddot{X}\}+[C]\{\dot{X}\}+[K]\{X\}+\{F\}=u(t)
$$

Consider that the first step is to define the forces, $\{F\}$, that are provided due to the actuation. This vector has been defined in Appendix G. Leaving a detailed design of a control law to the future work, yet in order to demonstrate the authority of the actuation, the system's response to an impulse actuation is determined. Thus rewriting Eq. (5.2) for $u(t)=0$, one has,

$$
[M]\{\ddot{X}\}+[C]\{\dot{X}\}+[K]\{X\}=-\{F\}
$$




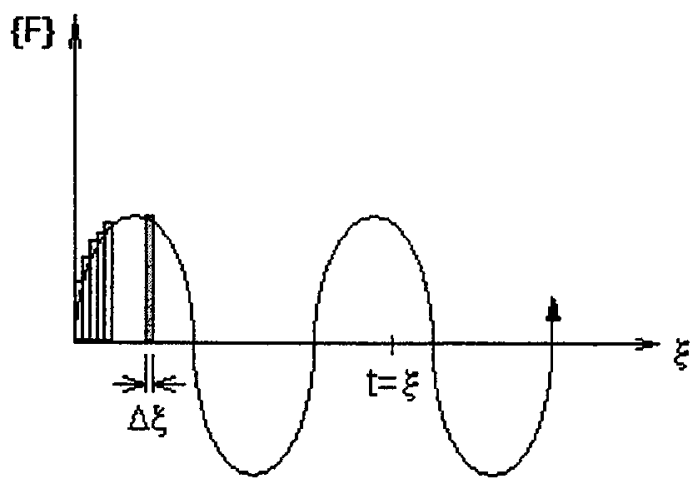

Figure 5.25: Sinusoidal input

In developing the control law, the actuation forces $\{F\}$ would be a function of an electric field that could vary, for example, as a sinusoidal function. Such a force can be represented as a series of impulses as shown in Figure 5.25, where time is represented by the variable $\xi$ and $\Delta \xi$ is the time step. For an infinitesimally small time step $\Delta \xi_{i}$, the explicit expression for Eq. (5.3) becomes

$$
[M]\{\ddot{X}\}+[C]\{\dot{X}\}+[K]\{X\}=-\{F\} \delta(t)
$$

where $\delta(t)$ is the Dirac-delta function.

The Runge-Kutta method can be used to solve for $v, w$, and $\phi$ which will show the system's behaviour to this impulsive field. Runge-Kutta is a numerical solver which requires only the solution at the immediately proceeding time point. Thus it requires that the equations be described in the following form:

$$
\begin{aligned}
& \dot{x}_{1}=x_{2} \\
& \dot{x}_{2}=-\frac{C}{M} x_{2}-\frac{K}{M} x_{1}-\frac{F}{M} \delta(t)
\end{aligned}
$$


where

$$
x_{1}=\left\{\begin{array}{l}
\bar{v} \\
\bar{w} \\
\phi
\end{array}\right\}
$$

The impulse response of the system under two different fields of $1800 \mathrm{~V}$ and $900 \mathrm{~V}$ are given in Figures 5.26 and 5.27, respectively. These graphs depict the lead-lag, flap, and torsional deflections at the blade tip due to impulsive actuation. Both graphs indicate a stiff response over time for the lead-lag direction and a quick decay rate in flap and torsion. However, zooming into the lead-lag graphs in Figures 5.28 and 5.29 shows that the maximum displacement is the same between the two voltages, but the lower voltage has a quicker decay rate. This indicates promising results for a full implementation of a smart blade. 

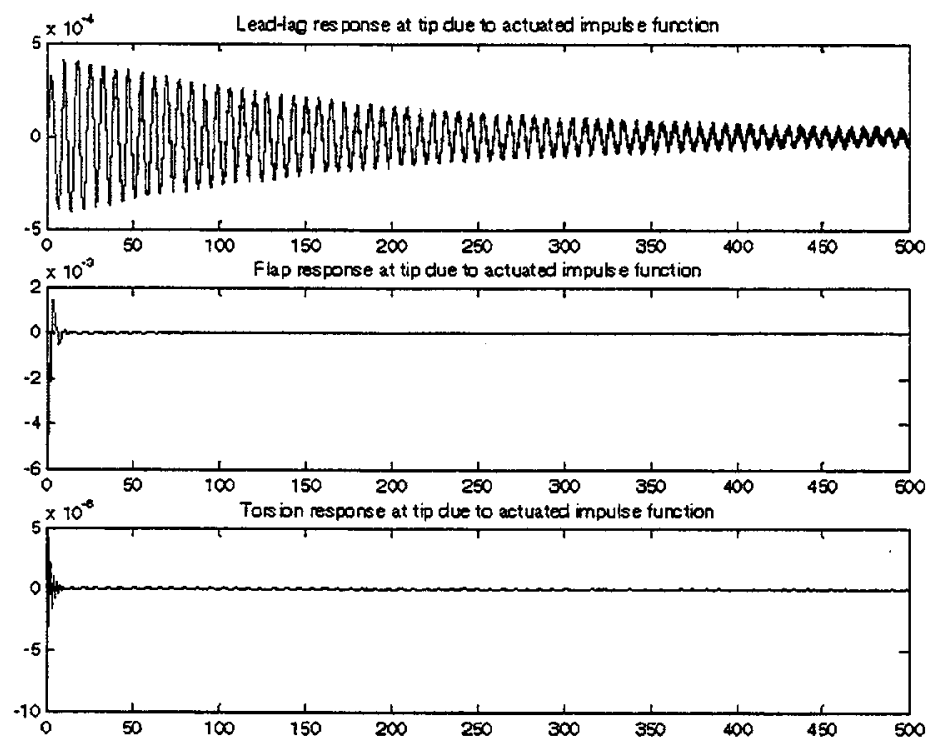

Figure 5.26: Response to a unit-impulse function at $1800 \mathrm{~V},\{F\} \delta(t)$
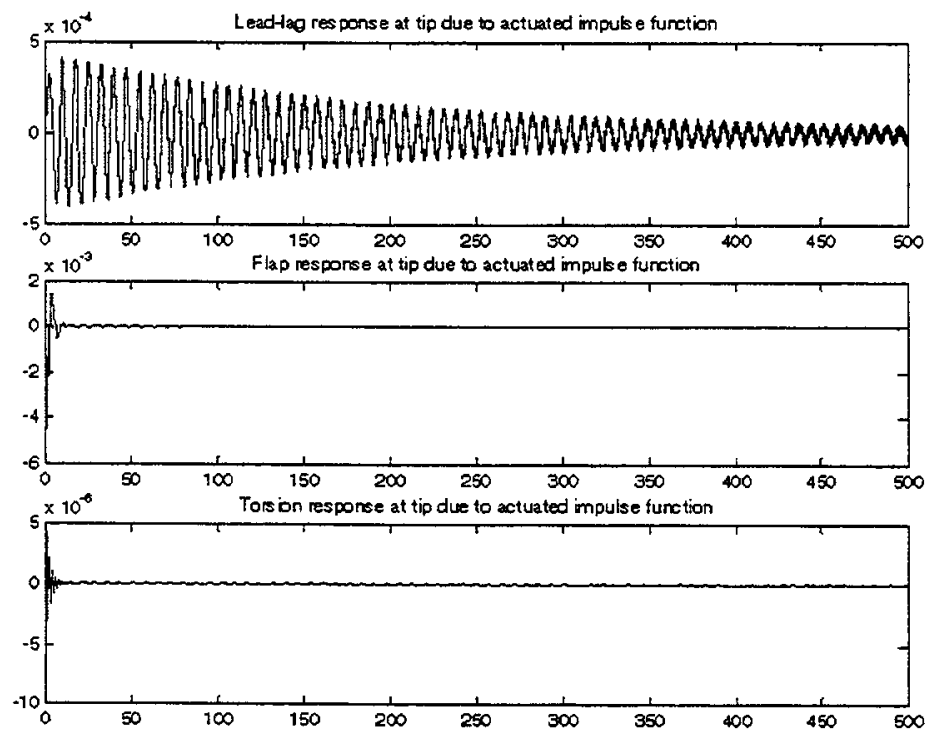

Figure 5.27: Response to a unit-impulse function at $900 \mathrm{~V},\{F\} \delta(t)$ 


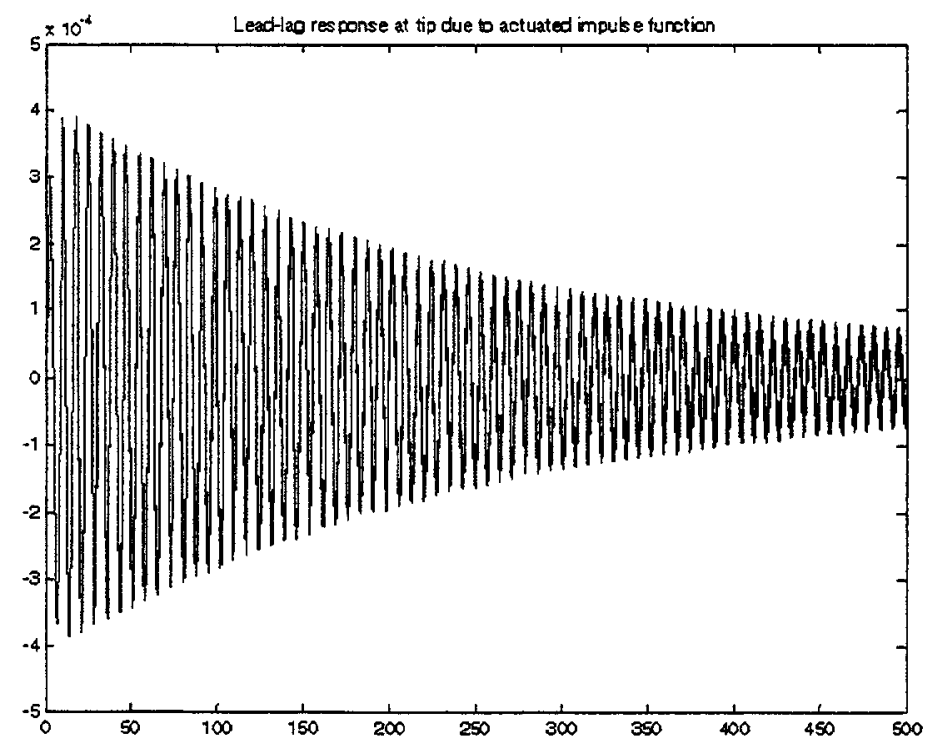

Figure 5.28: Response to a unit-impulse function at $1800 \mathrm{~V}$ for lead-lag

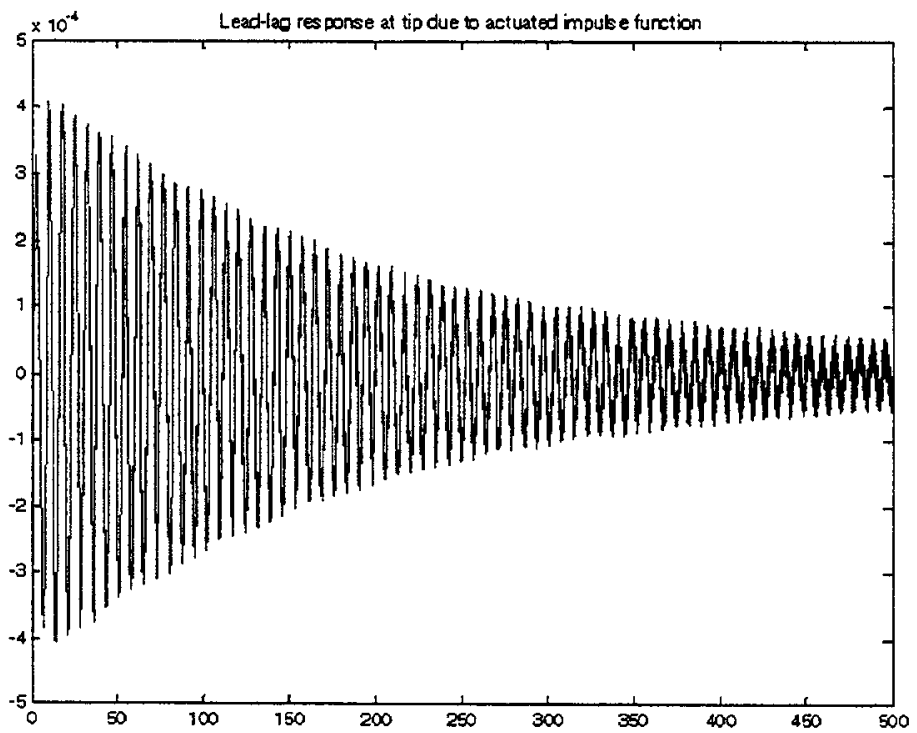

Figure 5.29: Response to a unit-impulse function at $900 \mathrm{~V}$ for lead-lag 


\section{Chapter 6}

\section{Summary, Conclusions and}

\section{Recommendations}

\subsection{Summary}

This thesis is intended to contribute to the solution for dampening helicopter vibrations stemming from the rotor blades by implementing individual blade control. The use of embedded piezoelectrics, called active fibre composites, as distributed actuators that are fully integrated into the structure is investigated and shown to be a promising solution that addresses the problem. This is accomplished by individually twisting each blade by direct strain actuation by means of electric currents. These preliminary results serve as a basis for future experimentation on the blade and the development of the control system that will complete the functionality of the blade system.

Three key areas were addressed:

1. The development of the analytical expressions to include the actuating elements for describing the dynamic equations of motion of a rotor blade in hover.

2. A study of the optimal blade design configuration. 
3. An analysis of the stability of the system.

As an initial attempt, the dynamics of rotor blades in hover only are considered. The corresponding analytical formulation is based on: a linear two-dimensional cross-sectional analysis, and a nonlinear one-dimensional beam dynamic formulation. The cross-sectional analysis is based on Badir's variational asymptotic method, which is an iterative process that utilises the shell energy, to approximate bending displacements and strain fields $[35,42]$. The elastic strain relationships of a blade are expanded to take into account the new strains caused by the piezoelectric effect. The strain energy expression is used to derive the constitutive relations that define the axial force, torsion, and bending moments experienced by the blade. The stiffness constants and actuation contributions that are a part of the constitutive relations are integrated into the structural component of the nonlinear beam formulation. This formulation of beam dynamics is based on further expanding the approach that is suggested by Hodges and Dowell, and Hodges and Ormiston where strain and kinetic energies of the system along with the work done by non-conservative forces are incorporated into Hamilton's method [40,41]. Galerkin's method is used to solve for the equations of motion of the rotor blade in lead-lag, flap, and torsion. Thus, steady-state and perturbation quantities for not only the passive blade, but now also the active blade are determined.

The study of the optimal design configuration is performed by basing the design on a scaled model of the BO105 helicopter. Tuning the rotor blade frequencies to a typical rotor blade will ensure that basic structural characteristics are maintained. This retention of accuracy is also maintained for aerodynamic properties by setting the Lock number. Three, two-celled, thin-walled anisotropic composite blade designs are found to sufficiently satisfy the constrained properties. In following the assumptions set out by the beam formulation these blades are modelled as long, straight, slender, cantilevered beams that undergo moderate displacements. Analyses are carried out for the smart blade by varying pitch 
angle and length of blade actuated.

An active blade must show stability levels equal to or better than the passive and nonactuated blades. Stability is indicated by the eigenvalues or the natural frequencies of the homogeneous equations of motion. These eigenvalues, for the active blades undergoing a DC current and the non-active blades, are solved directly. For periodic actuation with a sinusoidal or AC current, the eigenvalues must be obtained through the Floquet method. The modes of interest for this application are the 1st lead-lag, 1st and 2nd flap, and torsion where instabilities like divergence or flutter should not be present. Finally, in anticipation of future work, the transfer function of the smart blade is determined from the system response to a unit impulsive actuation. Thus, the basis for the smart blade is established and the structural properties are defined such that future implementation of a complete feedback system can be carried out.

\subsection{Conclusions}

Several conclusions can be drawn from the present research. They are as follows:

- The active fibre composite shows that it can achieve tip twists on the order of magnitude achieved by previous researchers. The final design exhibits a maximum twist of approximately $1.8^{\circ}$ for two active plies.

- All three designs studied show good stability levels and no indications of divergence or flutter for the modes studied and for both the actuated and non-actuated scenarios.

- Increasing the pitch angle between 0.0 and $0.3 \mathrm{rad}$ generally increases the magnitudes of deflection and torsion.

- Increasing the number of active plies from 2 to 4 shows trends of increasing the level of twist, but decreasing the magnitude of bending in lead-lag and flap. A blade with 
actuators embedded along the full length of the span, rather than some portion, provides consistent actuation levels.

- The validation studies show that the computational code developed in MATLAB is a very suitable code for designing $\mathrm{AFC}$ blades and stability analysis of such blades.

- The linear beam formulation given in Appendix $\mathrm{C}$ shows limitations.

\subsection{Recommendations}

- Testing on the rotor blade in hover is needed to validate the analytical results. This should include a Mach-scaled version of the blade model for wind-tunnel testing.

- Carrying out an analysis of the rotor blade in forward flight is required as the next logical progression for expanding the equations of motion. This would signify developing the kinetic energy and aerodynamic terms to account for periodic coefficients.

- Develop a feedback control system for the smart rotor blade. This should include an analysis of the applied AC current for counteracting the periodic perturbations. Simulations for various perturbations and excitations should also be included. These perturbations may be mathematically represented by step, impulse, or ramp functions.

- Vary the inboard and outboard coupling parameter, $\Re$, to determine its relative importance. It is known that lead-lag deflection is influenced by $\Re$ due to flap-lag structural coupling.

- Determine the effects of droop, sweep, pre-twist and torque offset. The precone angle, $\beta_{p c}$, should also be investigated as it is known to have a destabilizing effect for lead-lag and torsion frequencies. 
- Refine the blade design to improve the lead-lag stiffness, $\mathrm{EI}_{z}$. The addition of nonuniformities in stiffness, mass, and torsional rigidity throughout the blade span and cross-section may help to refine the blade design. Also improvements can be made in narrowing the gap in the chord-wise offsets between the elastic and mass, aerodynamic, and tension axes.

- Apply a more complex formulation to adequately account for blade-vortex interactions, nonlinearities at low Reynold's numbers, and turbulence. The aerodynamic analysis for the blade in hover is based on Greenberg's extension of Theodorsen's theory for a $2 \mathrm{D}$ airfoil. This is a simple approximation for a blade undergoing motion in incompressible flow.

- Perform a study of the applied voltage. This should be done both experimentally and analytically for ranges in voltage between 0 and $2000 \mathrm{~V}$. Results from various researchers for tip twist are varied, and the applied voltage is known to be a sensitive matter especially in manufacturing. 


\section{References}

[1] R.G. Loewy. Helicopter vibrations: A technological perspective. 40th Annual Forum of the American Helicopter Society, Arlington, VA, pages 4-30, May 1984.

[2] P.P. Friedmann. Rotary-wing aeroelasticity with application to vtol vehicles. AIAA Journal, AIAA-90-1115-CP, pages 1624-69, 1990.

[3] J.T. Pearson, R.M. Goodall, and I. Lyndon. Active control of helicopter vibration. Computing 86 Control Engineering Journal, pages 277-84, December 1994.

[4] R.L. Bielawa. Rotory Wing Structural Dynamics and Aeroelasticity. AIAA Education Series, Washington, DC, 1992.

[5] J. Shaw and N. Albion. Active control of the helicopter rotor for vibration reduction. Journal of the American Helicopter Society, pages 32-9, July 1981.

[6] T. Lee and I. Chopra. Design of piezostack-driven trailing-edge flap actuator for helicopter rotors. Journal of Smart Materials and Structures, 10:15-24, 2001.

[7] A.D. Jacot. Smart structures for rotorcraft control (ssrc). SPIE Vol. 3044, pages 114-22, 1997.

[8] F.K. Straub, H.T. Ngo, V. Anand, and D.B. Domzalski. Development of a piezoelectric actuator for trailing edge flap control of full scale rotor blades. Journal of Smart Materials and Structures, 10:25-34, 2001. 
[9] F.K. Straub. A feasibility study of using smart materials for rotor control. Journal of Smart Materials and Structures, 5:1-10, 1996.

[10] F. Nitzsche, A. Grewal, and D. Zimcik. Structural component having means for actively varying its stiffness to control vibrations. U.S. Patent No. 5973440 . European Patent EP-996570-B1, 1999.

[11] F. Nitzsche and E.J. Breitbach. Using adaptive structures to attenuate rotary wing aeroelastic response. AIAA, Journal of Aircraft, 31(5):1178-88, 1994.

[12] F. Nitzsche. Designing an active impedance control device to actively control helicopter blade vibration. CEAS/AIAA International Forum on Aeroelasticity and Structural Dynamics, Madrid, Spain, 3, 2001.

[13] S. Solaiman. Dynamic modelling and stability analysis of a smart hingeless helicopter blade. Master's thesis, Carleton University, Ottawa, ON, September 1999.

[14] N. Morozova. Dynamic stability analysis of a helicopter blade with adaptive damper. Master's thesis, Carleton University, Ottawa, ON, Sept 2002.

[15] J.P. Narkiewicz and G.T.S. Done. Overview of smart structure concepts for helicopter rotor control. Presented at the 2nd European Conf. on Smart Structures and Materials, Glasgow, pages 242-45, 1994.

[16] H. Strehlow and H. Rapp. Smart materials for helicopter rotor active control. AGARD/SMP Specialist's Meeting on Smart Structures for Aircraft and Spacecraft, 5:1-16, October 5-7 1992.

[17] W. Wilkie, W. Belvin, and K.C. Park. Aeroelastic analysis of helicopter rotor blades incorporating anisotropic piezoelectric twist actuation. ASME 1996 World Congress and Exposition, 1996. 
[18] E.F. Crawley and J. de Luis. Use of piezoelectric actuators as elements of intelligent structures. AIAA Journal, 25(10):1373-85, 1987.

[19] E.F. Crawley and K.B. Lazarus. Induced strain actuation of isotropic and anisotropic plates. AIAA Journal, 29(6):944-51, June 1991.

[20] R.W. Rehfield. Design analysis methodology for composite rotor blades. Proceedings of the Seventh DoD/NASA Conference on Fibrous Composites in Structural Design, Denver, CO, Grant NAG-2-238, 1985.

[21] L.W. Rehfield and A.R. Atilgan. Shear center and elastic axis and their usefulness for composite thin-walled beams. Proceedings of the American Society for Composites, Fourth Technical Conference, Blacksburg, Va, pages 179-88, October 3-5 1989.

[22] R. Barrett. Actuation strain decoupling through enhanced directional attachment in plates and aerodynamic surfaces. Proceedings of the 1st European Conference on Smart Structures and Materials, Glasgow, pages 383-86, 1992.

[23] P.C. Chen and I. Chopra. A feasibility study to build a smart rotor: induced-strain actuation of airfoil twisting using piezoceramic crystals. Proceedings of the Society of Photo-Optical and Instrumentation Engineering, San Diego, CA, 1917:238-54, 1993.

[24] P.C. Chen and I. Chopra. Induced strain actuation of composite beams and rotor blades ith embedded piezoceramic elements. Journal of Smart Materials and Structures, 3(5):35-48, 1996.

[25] P.C. Chen and I. Chopra. Hover testing of smart rotor with induced-strain actuation of blade twist. AIAA Journal, 35(1):6-16, January 1997.

[26] A.A. Bent. Piezoelectric fiber composites for structural actuation. Master's thesis, MIT, Cambridge, MA, January 1994. 
[27] B.Z. Janos and N.W. Hagood. Overview of active fiber composites technologies. Active Materials and Structures Laboratory, MIT, Cambridge, MA, 1998.

[28] A.J. DuPlessis. Modeling and experimental testing of twist actuated single cell composite beams for helicopter blade control. Master's thesis, MIT, Cambridge, MA, February 1996. AMSL \#96-1.

[29] A.A. Bent, N.W. Hagood, and J.P. Rodgers. Anisotropic actuation with piezoelectric fiber composites. Journal of Intelligent Material Systems and Structures, 6:338-49, May 1995.

[30] J.P. Rodgers and N.W. Hagood. Manufacture of adaptive composite plates incorporating piezoelectric fiber composite plies. Proceedings of the 36th AIAA/ASME/ASCE/AHS/ASC Structures, Structural Dynamics, and Materials Conference, New Orleans, LA, April 1995.

[31] R.C. Derham and N.W. Hagood. Rotor design using smart materials to actively twist blades. Proceedings of the American Helicopter Society 52nd Annual Forum, Washington, DC, pages 1242-52, 1996.

[32] J.P. Rodgers and N.W. Hagood. Preliminary mach-scale hover testing of an integral twist-actuated rotor blade. SPIE, 3329:291-308.

[33] J.P. Rodgers and N.W. Hagood. Hover testing of a 1/6th mach-scale ch-47d blade with integral twist actuation. Presented at the 9th International Conference on Adaptive Structures and Technology, Cambridge, MA, 1998.

[34] V.L. Berdichevskii. Variational-asymptotic method of constructing a theory of shells. PMM - Journal of Applied Mathematics \& Mechanics, 43(4):711-36, 1979. 
[35] A.M. Badir. Analysis of advanced thin-walled composite structures. Doctoral thesis, Georgia Institute of Technology, Atlanta, GA, February 1992.

[36] C.E.S. Cesnik and S.J. Shin. Structural analysis for designing rotor blades with integral actuators. 39th Structures, Structural Dynamics, and Materials Conference, AIAA, April 20-23 1998.

[37] S.J. Shin. Design, manufacturing and testing of an active twist rotor. Master's thesis, MIT, Cambridge, MA, June 1999. AMSL \#99-3.

[38] B. Bailey. Investigation of a composite hingeless helicopter rotor blade with integral actuators. Master's thesis, Carleton University, Ottawa, ON, April 2000.

[39] X. Shang and D.H. Hodges. Aeroelastic stability of composite rotor blades in hover. Proceedings of the 36th Structures, Structural Dynamics and Materials Conference, New Orleans, LA, 10-12 April, 1995.

[40] D.H. Hodges and E.H. Dowell. Nonlinear equations of motion for the elastic bending and torsion of twisted nonuniform rotor blades. Contract Report, NASA TN D-7818, December 1974.

[41] D.H. Hodges and R.A. Ormiston. Stability of elastic bending and torsion of uniform cantilever rotor blades in hover with variable structural coupling. Contract Report, NASA TN D-8192, April 1976.

[42] A.M. Badir. Analysis of two-cell composite beams. Proceedings of the 36th Structures, Structural Dynamics, and Materials Conference, AIAA, pages 419-24, 1995.

[43] R. Chandra, A.D. Stemple, and I. Chopra. Thin-walled composite beams under bending, torsional, and extensional loads. Journal of Aircraft, 27(7):619-26, July 1990. 
[44] E.C. Smith and I. Chopra. Formulation and evaluation of an analytical model for composite box-beams. Proceedings of the 31st Structures, Structural Dynamics and Materials Conference, Long Beach, Ca, April 2-4, AIAA Journal, pages 759-82, 1990.

[45] L.W. Rehfield, A.R. Atilgan, and D.H. Hodges. Nonclassical behavior of thin-walled composite beams with closed cross sections. Journal of the American Helicopter Society. Also submitted to Proceedings of the American Helicopter Society National Specialists' Meeting on Advanced Rotorcraft Structures, Williamsburg, Va, pages 4250, May 1990.

[46] W.B. Bickford. Advanced Mechanics of Materials. Prentice Hall, Upper Saddle River, NJ, 1998.

[47] R.M. Jones. Mechanics of Composite Materials. Scripta Book Company, Washington, DC, 1975.

[48] J.R. Vinson and R.L. Sierakowski. The behavior of structures composed of composite materials. Martinus Nijhoff Publishers, Dordrecht, 1986.

[49] R.F. Gibson. Principles of Composite Material Mechanics. McGraw Hill, Inc., New York, 1994.

[50] R.L. Bisplinghoff. Aeroelasticity. Addison-Wesley Pub. Co., Cambridge, MA, 1955.

[51] T. Chang and R.R. Craig Jr. On normal modes of uniform beams. EMRL 1068, The University of Texis at Austin, Jan 1969.

[52] Jane's all the world's aircraft 2002-2003. Jane's Information Group Ltd., Surrey, UK, 2002. 
[53] Sungsoo Na. Control of Dynamic Response of Thin-Walled Composite Beams using Structural Tailoring and Piezoelectric Actuation. Doctor of philosophy, Virginia Tech, Blacksburg, VA, 1997.

[54] D.H. Hodges. Review of composite rotor blade modeling. AIAA Journal, 28(3):56165, March 1990.

[55] E.H. Mansfield and A.J. Sobey. The fibre composite helicopter blade, part 1: Stiffness properties, part 2: Prospects for aeroelastic tailoring. Aeronautical Quarterly, 30(2), 1979.

[56] C.E.S. Cesnik and D.H. Hodges. Vabs: A new concept for composite rotor blade cross-sectional modeling. AHS, 51st Annual Forum, Fort Worth, TX, pages 27-38, May 9-11 1995.

[57] W.R. Splettstoesser et. al. The helinoise aeroacoustic rotor test in the dnw - test documentation and representative results. DLR - Mitt. 93-09, DLR, Braunschweig, Germany, 1993.

[58] R.E.D. Bishop and D.C. Johnson. The mechanics of vibration. Cambridge [Eng.] University Press, Cambridge, 1960.

[59] D.A. Peters and K.H. Hohenemser. Application of the floquet transition matrix to problems of lifting rotor stability. Journal of the American Helicopter Society, 16(2):25-33, April 1971.

[60] J. Dugundji and J.H. Wendell. Some analysis methods for rotating systems with periodic coefficients. AIAA Journal, 21(6):890-97, June 1982.

[61] Y.C. Fung. An Introduction to the Theory of Aeroelasticity. Dover Publications, Inc., New York, 1993. 


\section{Appendix A}

\section{Ordering Schemes}

\section{A.1 2D Cross-Sectional Analysis}

Detailed ordering expressions for the main variables and equations of Chapter 2 are provided here.

Orders for the projections of the position vector $r$ on $t$ and $n$ are,

$$
r_{n} \sim O(d) \text { and } r_{t} \sim O(d)
$$

The dominant orders of strain $\gamma_{i j}$ and rate of curvature $\rho_{i j}$ expressions are evaluated at zeroth-order. First and second order equations are found similarly.

$$
\begin{aligned}
\gamma_{11} & =\frac{\partial v_{l}}{\partial x} \sim O\left(\frac{\Delta d}{L^{2}}\right) \\
2 \gamma_{12} & =\frac{\partial v_{l}}{\partial s}+\frac{\partial v_{s}}{\partial x} \sim O\left(\frac{\Delta}{L}\right) \\
\gamma_{22} & =\frac{\partial v_{s}}{\partial s}+\frac{v_{\xi}}{R} \sim O\left(\frac{\Delta}{d}\right) \\
\rho_{11} & =\frac{\partial^{2} v_{\xi}}{\partial x^{2}} \sim O\left(\frac{\Delta}{L^{2}}\right) \\
\rho_{12} & =\frac{\partial^{2} v_{\xi}}{\partial x \partial s}+\frac{1}{4 R}\left(\frac{\partial v_{l}}{\partial s}-3 \frac{\partial v_{s}}{\partial x}\right) \sim O\left(\frac{\Delta}{L d}\right)
\end{aligned}
$$




$$
\rho_{22}=\frac{\partial^{2} v_{\xi}}{\partial s^{2}}-\frac{\partial}{\partial s}\left(\frac{v_{s}}{R}\right) \sim O\left(\frac{\Delta}{d^{2}}\right)
$$

The electromechanical strain, $d_{e \alpha \beta} E_{e}$ is a degree smaller than the highest order of the strains, i.e. $\gamma_{11}$, thus,

$$
d_{e \alpha \beta} E_{e} \sim O\left(\frac{\Delta d}{L^{2}}\right) \times O\left(\frac{d}{L}\right) \sim O\left(\frac{\Delta d^{2}}{L^{3}}\right)
$$

Order analyses for the energy expressions are as follows:

a) for the shell energy per unit area,

$$
\Phi \sim O\left(\frac{E \Delta^{2} h}{d^{2}}\right)
$$

b) for the work done by external forces,

$$
P_{i} u_{i} \sim O\left(\frac{E \Delta^{2} h}{L^{2}}\right)
$$

since

$$
u_{i} \sim O(\Delta), \quad P_{i} \sim O\left(\frac{E \Delta^{2} h}{L^{2}}\right)
$$

however, because

$$
\frac{1}{L^{2}} \ll \frac{1}{d^{2}}
$$

then

$$
P_{i} u_{i} \ll \Phi
$$

so work done by external forces is neglected.

The orders of the average cross-sectional translations and torsional rotations are,

$$
U_{1} \sim O\left(\frac{\Delta d}{L}\right), \quad U_{2} \sim O(\Delta), \quad U_{3} \sim O(\Delta), \quad \varphi \sim O\left(\frac{\Delta}{L}\right)
$$


The orders of the correction factors or warping terms from the first order approximation are,

$$
w_{i} \sim O\left(\frac{\Delta d}{L}\right)
$$

for $i=1,2,3$, and from the second order approximation,

$$
\bar{w}_{i} \sim O\left(\frac{\Delta d^{2}}{L^{2}}\right)
$$

for $i=4,5,6$.

Variables from the equations for the displacement approximations are,

$$
g_{1}(s) \sim O(d), \quad g_{2}(s), g_{3}(s) \sim O\left(d^{2}\right), \quad G(s) \sim O\left(d^{2}\right)
$$

The axial, coupling and shear stiffness constants have orders of magnitude of,

$$
\begin{array}{ll}
A \sim O(E h) & A^{(a)} \sim O\left(\frac{E \Delta d^{2} h}{L^{3}}\right) \\
B \sim O(E h) & C^{(a)} \sim O\left(\frac{E \Delta d^{2} h}{L^{3}}\right) \\
C \sim O(E h) &
\end{array}
$$

The orders belonging to the final stiffness constants of the stiffness matrix $[K]$ are,

$$
\begin{aligned}
& K_{11} \sim O(E d h) \quad K_{23} \sim O\left(E d^{3} h\right) \\
& K_{12} \sim O\left(E d^{2} h\right) \quad K_{24} \sim O\left(E d^{3} h\right) \\
& K_{13} \sim O\left(E d^{2} h\right) \quad K_{33} \sim O\left(E d^{3} h\right) \\
& K_{14} \sim O\left(E d^{2} h\right) \quad K_{34} \sim O\left(E d^{3} h\right) \\
& K_{22} \sim O\left(E d^{3} h\right) \quad K_{44} \sim O\left(E d^{3} h\right)
\end{aligned}
$$


where the orders of the actuation vector are,

$$
\begin{aligned}
& F_{1}^{(a)} \sim O\left(\frac{E \Delta d^{3} h}{L^{3}}\right) \\
& M_{1}^{(a)} \sim O\left(\frac{E \Delta d^{4} h}{L^{3}}\right) \\
& M_{2}^{(a)} \sim O\left(\frac{E \Delta d^{4} h}{L^{3}}\right) \\
& M_{3}^{(a)} \sim O\left(\frac{E \Delta d^{4} h}{L^{3}}\right)
\end{aligned}
$$

\section{A.2 1D Beam Formulation}

The following set of variables and their order apply to those deflection, coordinates and warping terms of Chapter 3.

$$
\begin{aligned}
\frac{u}{L} & \sim O\left(\underline{\varepsilon}^{2}\right) & \frac{t}{L} & \sim O(\underline{\varepsilon}) \\
\frac{v}{L} & \sim O(\underline{\varepsilon}) & \frac{\eta}{L} & \sim O(\underline{\varepsilon}) \\
\frac{w}{L} & \sim O(\underline{\varepsilon}) & \frac{\zeta}{L} & \sim O(\underline{\varepsilon}) \\
\phi & \sim O(\underline{\varepsilon}) & \frac{\lambda}{L^{2}} & \sim O\left(\underline{\varepsilon}^{2}\right) \\
\frac{x}{L} & \sim O(1) & \frac{\partial \lambda / \partial \eta}{L} & \sim O(\underline{\varepsilon}) \\
\frac{c}{L} & \sim O(\underline{\varepsilon}) & \frac{\partial \lambda / \partial \zeta}{L} & \sim O(\underline{\varepsilon})
\end{aligned}
$$




\section{Appendix B}

\section{Cross-Sectional Constants}

Variables utilized in the displacement fields of the variational asymptotic analysis of Chapter 2 are provided here. Beginning with $a_{i}$,

$$
\begin{aligned}
& a_{1}=-\frac{c_{3}}{c_{1}} \\
& a_{2}=\frac{\left(b_{1}+b_{2}\right) a_{1}+b_{4}}{b_{2}} \\
& a_{3}=-\frac{c_{2}}{c_{1}} \\
& a_{4}=\frac{\left(b_{1}+b_{2}\right) a_{3}+b_{3}}{b_{2}} \\
& a_{5}=\frac{b_{5} \oint_{I} y b(s) d s+b_{2} d_{1}}{e_{1}} \\
& a_{6}=\frac{b_{1}+b_{2}}{b_{2}} a_{5}-\frac{\oint_{I} y b(s) d s}{b_{2}} \\
& a_{7}=\frac{b_{5} \oint_{I} z b(s) d s+b_{2} d_{2}}{e_{1}} \\
& a_{8}=\frac{b_{1}+b_{2}}{b_{2}} a_{7}-\frac{\oint_{I} z b(s) d s}{b_{2}}
\end{aligned}
$$

The following intra-relationships between $a$ variables exist also, they are, 


$$
\begin{array}{ll}
a_{5}=-a_{3} y & a_{5} z=a_{7} y \\
a_{6}=-a_{4} y & a_{6} z=a_{8} y \\
a_{7}=-a_{3} z & \\
a_{8}=-a_{4} z &
\end{array}
$$

The remaining expressions for $b_{i}, c_{i}, d_{i}$, and $e_{i}$ are,

$$
\begin{aligned}
b_{1}= & 4 \int_{0}^{s_{1}} c(s) d s \\
b_{2}= & 4 \int_{s_{1}}^{s_{2}} c(s) d s \\
b_{3}= & \oint_{I} b(s) d s \\
b_{4}= & -\oint_{I} r_{n} d s=-2 A_{e I} \\
b_{5}= & 4 \int_{s_{2}}^{s_{3}} c(s) d s \\
b_{6}= & \oint_{I I} b(s) d s \\
b_{7}= & -\oint_{I I} r_{n} d s=-2 A_{e I I} \\
& b_{3} \cdot b_{7}=b_{4} \cdot b_{6} \\
d_{1} & =\oint_{I+I I} y b(s) d s \\
d_{2} & =\oint_{I+I I} z b(s) d s \\
c_{1}= & b_{1}+\frac{\left(b_{1}+b_{2}\right) b_{5}}{b_{2}} \\
c_{2}= & b_{3}+b_{6}+\frac{b_{3} b_{5}}{b_{2}} \\
c_{3}= & b_{4}+b_{7}+\frac{b_{4} b_{5}}{b_{2}}
\end{aligned}
$$




$$
e_{1}=b_{1} b_{2}+\left(b_{1}+b_{2}\right) b_{5}
$$

where $A_{e I}$ and $A_{e I I}$ are the enclosed areas of each cell.

The expressions for variables that are a function of the applied electric field are,

$$
\begin{aligned}
& f_{1}^{(a)}=\oint_{I} c(s) C^{(a)} d s \\
& f_{2}^{(a)}=\oint_{I I} c(s) C^{(a)} d s
\end{aligned}
$$

$$
\begin{aligned}
& g_{1}^{(a)}=2 \frac{\left(b_{5}+b_{2}\right) f_{1}^{(a)}+b_{2} f_{2}^{(a)}}{\left(b_{1}+b_{2}\right)\left(b_{2}+b_{5}\right)-b_{2}^{2}} \\
& g_{2}^{(a)}=2 \frac{\left(b_{1}+b_{2}\right) f_{2}^{(a)}+b_{2} f_{1}^{(a)}}{\left(b_{1}+b_{2}\right)\left(b_{2}+b_{5}\right)-b_{2}^{2}}
\end{aligned}
$$

The variables that specifically apply to the left branch $\left(s=0 \rightarrow s_{1}\right)$ of the blade cross-section are evaluated as follows,

$$
\begin{aligned}
G(s) & =\int_{0}^{s_{1}}\left[4 a_{1} c(s)-r_{n}\right] d s \\
g_{1}(s) & =\int_{0}^{s_{1}}\left[b(s)+4 a_{3} c(s)\right] d(s) \\
g_{2}(s) & =-\int_{0}^{s_{1}}\left[b(s) y(s)-4 a_{5} c(s)\right] d(s) \\
g_{3}(s) & =-\int_{0}^{s_{1}}\left[b(s) z(s)-4 a_{7} c(s)\right] d(s) \\
v_{1}^{(a)} & =\int_{0}^{s_{1}}\left[2 g_{1}^{(a)}-C^{(a)}(s)\right] c(s) d s
\end{aligned}
$$

the web $\left(s=s_{1} \rightarrow s_{2}\right)$ as follows,

$$
\begin{aligned}
& G(s)=\int_{s_{1}}^{s_{2}}\left[4\left(a_{1}-a 2\right) c(s)-r_{n}\right] d s \\
& g_{1}(s)=\int_{s_{1}}^{s_{2}}\left[b(s)+4\left(a_{3}-a_{4}\right) c(s)\right] d(s)
\end{aligned}
$$




$$
\begin{aligned}
g_{2}(s) & =-\int_{s_{1}}^{s_{2}}\left[b(s) y(s)-4\left(a_{5}-a_{6}\right) c(s)\right] d(s) \\
g_{3}(s) & =-\int_{s_{1}}^{s_{2}}\left[b(s) z(s)-4\left(a_{7}-a_{8}\right) c(s)\right] d(s) \\
v_{1}^{(a)} & =\int_{s_{1}}^{s_{2}}\left[2\left(g_{1}^{(a)}-g_{2}^{(a)}\right)-C^{(a)}(s)\right] c(s) d s
\end{aligned}
$$

the right branch $\left(s=s_{2} \rightarrow s_{3}\right)$ as follows,

$$
\begin{aligned}
G(s) & =\int_{s_{2}}^{s_{3}}\left[4 a_{2} c(s)-r_{n}\right] d s \\
g_{1}(s) & =\int_{s_{2}}^{s_{3}}\left[b(s)+4 a_{4} c(s)\right] d(s) \\
g_{2}(s) & =-\int_{s_{2}}^{s_{3}}\left[b(s) y(s)+4 a_{6} c(s)\right] d(s) \\
g_{3}(s) & =-\int_{s_{2}}^{s_{3}}\left[b(s) z(s)+4 a_{8} c(s)\right] d(s) \\
v_{1}^{(a)} & =\int_{s_{2}}^{s_{3}}\left[2 g_{2}^{(a)}-C^{(a)}(s)\right] c(s) d s
\end{aligned}
$$

The axial force from the constitutive relations is evaluated as,

$$
\begin{aligned}
F_{1} & =\oint \frac{\partial \Phi}{\partial U_{1}^{\prime}} d s \\
& =\oint\left(\frac{\partial \Phi}{\partial \gamma_{11}} \frac{\partial \gamma_{11}}{\partial U_{1}^{\prime}}+\frac{\partial \Phi}{\partial\left(2 \gamma_{12}\right)} \frac{\partial\left(2 \gamma_{12}\right)}{\partial U_{1}^{\prime}}\right) d s \\
& =\oint\left(\left(A \gamma_{11}-A^{(a)}+\frac{1}{2} B\left(2 \gamma_{12}\right)\right)+\left(\frac{1}{2} B \gamma_{11}+\frac{1}{4} C\left(2 \gamma_{12}\right)-\frac{1}{2} C^{(a)}\right)\left(\frac{d g_{1}}{d s}\right)\right) d s \\
& =K_{11} U_{1}^{\prime}+K_{12} \varphi^{\prime}-K_{13} U_{3}^{\prime \prime}+K_{14} U_{2}^{\prime \prime}-F_{1}^{(a)}
\end{aligned}
$$

and where the other components $M_{1}, M_{2}$ and $M_{3}$ are evaluated in the same fashion.

The stiffness coefficients $K_{i j}$ are a function of both cross-sectional geometry and material constants,

$$
K_{11}=\oint\left(A-\frac{B^{2}}{C}\right) d s-4 a_{3} \oint_{I} \frac{B}{C} d s-4 a_{4} \oint_{I I} \frac{B}{C} d s
$$




$$
\begin{aligned}
& K_{12}=-4 a_{1} \oint_{I} \frac{B}{C} d s-4 a_{2} \oint_{I I} \frac{B}{C} d s \\
& K_{13}=\oint\left(A-\frac{B^{2}}{C}\right) z d s+4 a_{7} \oint_{I} \frac{B}{C} d s+4 a_{8} \oint_{I I} \frac{B}{C} d s \\
& K_{14}=-\oint\left(A-\frac{B^{2}}{C}\right) y d s-4 a_{5} \oint_{I} \frac{B}{C} d s-4 a_{6} \oint_{I I} \frac{B}{C} d s \\
& K_{22}=-4 a_{1} A_{e I}-4 a_{2} A_{e I I} \\
& K_{23}=4 a_{7} A_{e I}+4 a_{8} A_{e I I} \\
& K_{24}=-4 a_{5} A_{e I}-4 a_{6} A_{e I I} \\
& K_{33}=\oint\left(A-\frac{B^{2}}{C}\right) z^{2} d s+4 a_{7} \oint_{I} \frac{B}{C} z d s+4 a_{8} \oint_{I I} \frac{B}{C} z d s \\
& K_{34}=-\oint\left(A-\frac{B^{2}}{C}\right) z y d s-4 a_{5} \oint_{I} \frac{B}{C} z d s-4 a_{6} \oint_{I I} \frac{B}{C} z d s \\
& K_{44}=\oint\left(A-\frac{B^{2}}{C}\right) y^{2} d s+4 a_{5} \oint_{I} \frac{B}{C} y d s+4 a_{6} \oint_{I I} \frac{B}{C} y d s
\end{aligned}
$$




\section{Appendix C}

\section{Linear Beam Formulation}

This appendix describes a linear analysis to obtain the equations of motion for a smart

blade in hover. It is based on using the strain energy of the $2 \mathrm{D}$ cross-sectional formulation originating from Badir [35], and joining it with the kinetic and aerodynamic energies of Hodges and Ormiston [41] to form Hamilton's equation,

$$
\int_{t_{1}}^{t_{2}}[\delta(U-T)-\delta W] d t=0
$$

To begin with the variation of strain energy that can be found from Eq. (2.16), one has,

$$
\delta U=\int_{0}^{L}\left(F_{1} \delta U_{1}^{\prime}+M_{1} \delta \varphi^{\prime}-M_{2} \delta U_{3}^{\prime \prime}+M_{3} \delta U_{2}^{\prime \prime}\right) d x
$$

where these expressions may be integrated to give,

$$
\begin{aligned}
\int_{0}^{L} F_{1} \delta U_{1}^{\prime} d x & =\left.F_{1} \delta U_{1}\right|_{0} ^{L}-\int_{0}^{L} F_{1}^{\prime} \delta U_{1} d x \\
\int_{0}^{L} M_{1} \delta \varphi^{\prime} d x & =\left.M_{1} \delta \varphi\right|_{0} ^{L}-\int_{0}^{L} M_{1}^{\prime} \delta \varphi d x \\
\int_{0}^{L} M_{2} \delta U_{3}^{\prime \prime} d x & =-\left.M_{2} \delta U_{3}^{\prime}\right|_{0} ^{L}+\left.M_{2}^{\prime} \delta U_{3}\right|_{0} ^{L}-\int_{0}^{L} M_{2}^{\prime \prime} \delta U_{3} d x
\end{aligned}
$$




$$
\int_{0}^{L} M_{3} \delta U_{2}^{\prime \prime} d x=\left.M_{3} \delta U_{2}^{\prime}\right|_{0} ^{L}-\left.M_{3}^{\prime} \delta U_{2}\right|_{0} ^{L}+\int_{0}^{L} M_{3}^{\prime \prime} \delta U_{2} d x
$$

The boundary conditions from the expressions in Eq. (C.3) can be separated into one equation as $b(U)$ such that,

$$
b(U)=\left.F_{1} \delta U_{1}\right|_{0} ^{L}+\left.M_{1} \delta \varphi\right|_{0} ^{L}-\left.M_{2} \delta U_{3}^{\prime}\right|_{0} ^{L}+\left.M_{2}^{\prime} \delta U_{3}\right|_{0} ^{L}+\left.M_{3} \delta U_{2}^{\prime}\right|_{0} ^{L}-\left.M_{3}^{\prime} \delta U_{2}\right|_{0} ^{L}
$$

whereas the remaining integral terms have to be expressed in terms of the displacements $u, v$, and $w$ of Hodges and Ormiston rather than the cross-sectional displacements $U_{1}, U_{2}$, and $U_{3}$ of Badir1. This requires a transformation of elastic displacements as expressed in one coordinate system to the other. Figures 2.1, 3.2 and 3.3 are used to illustrate the beam deformation along the $x, y$, and $z$ axes. For example, the first integral of Eq. (C.3), i.e. $-\int_{0}^{L} F_{1}^{\prime} \delta U_{1} d x$, containing $U_{1}$ for axial displacement, may be transformed to the $u, v$, and $w$ coordinate system and the variation for $U_{1}$ found to be,

$$
\begin{aligned}
U_{1}(x) & =\left.u_{x}\right|_{\eta=\zeta=0}=u-v_{1}^{(a)}(s) \\
\delta U_{1}(x) & =\left.\delta u_{x}\right|_{\eta=\zeta=0}=\delta u \\
& =\frac{\partial U_{1}}{\partial u} \delta u=(1) \delta u
\end{aligned}
$$

where the following expressions define the relationships for all displacements in Figure 3.3,

$$
\begin{aligned}
U_{1}(x) & =u \\
U_{2}(x) & =v \cos \theta+w \sin \theta \\
U_{3}(x) & =-v \sin \theta+w \cos \theta \\
\delta U_{1}(x) & =\delta u \\
\delta U_{2}(x) & =\delta v \cos \theta+\delta w \sin \theta
\end{aligned}
$$




$$
\begin{aligned}
\delta U_{3}(x) & =-\delta v \sin \theta+\delta w \cos \theta \\
\varphi & =\phi
\end{aligned}
$$

Therefore the integrals of the strain relationships in Eq. (C.3) may be rewritten in terms of displacements defined by Hodges and Ormiston,

$$
\begin{aligned}
-\int_{0}^{L} F_{1}^{\prime} \delta U_{1} d x & =-\int_{0}^{L} F_{1}^{\prime} \delta u d x \\
-\int_{0}^{L} M_{1}^{\prime} \delta \varphi d x & =-\int_{0}^{L} M_{1}^{\prime} \delta \phi d x \\
-\int_{0}^{L} M_{2}^{\prime \prime} \delta U_{3} d x & =-\int_{0}^{L} M_{2}^{\prime \prime}(-\delta v \sin \theta+\delta w \cos \theta) d x \\
\int_{0}^{L} M_{3}^{\prime \prime} \delta U_{2} d x & =\int_{0}^{L} M_{3}^{\prime \prime}(\delta v \cos \theta+\delta w \sin \theta) d x
\end{aligned}
$$

which results in a new expression for the variation of strain energy of Eq. (C.2). This variation becomes,

$$
\begin{aligned}
\delta U= & -\int_{0}^{L} F_{1}^{\prime} \delta u d x-\int_{0}^{L} M_{1}^{\prime} \delta \phi d x+\int_{0}^{L_{1}}\left(M_{2}^{\prime \prime} \sin \theta+M_{3}^{\prime \prime} \cos \theta\right) \delta v d x \\
& +\int_{0}^{L}\left(M_{3}^{\prime \prime} \sin \theta-M_{2}^{\prime \prime} \cos \theta\right) \delta w d x
\end{aligned}
$$

Derivatives for the force, $F_{1}$, and moments, $M_{1}, M_{2}$, and $M_{3}$ acting on the beam are found from the constitutive expression, Eq. (2.26). They are,

$$
\begin{aligned}
F_{1}^{\prime} & =K_{11} U_{1}^{\prime \prime}+K_{12} \phi^{\prime \prime}-K_{13} U_{3}^{\prime \prime \prime}+K_{14} U_{2}^{\prime \prime \prime}-\left(F_{1}^{(a)}\right)^{\prime} \\
M_{1}^{\prime} & =K_{12} U_{1}^{\prime \prime}+K_{22} \phi^{\prime \prime}-K_{23} U_{3}^{\prime \prime \prime}+K_{24} U_{2}^{\prime \prime \prime}-\left(M_{1}^{(a)}\right)^{\prime} \\
M_{2}^{\prime \prime} & =K_{13} U_{1}^{\prime \prime \prime}+K_{23} \phi^{\prime \prime \prime}-K_{33} U_{3}^{\prime \prime \prime \prime}+K_{34} U_{2}^{\prime \prime \prime \prime}-\left(M_{2}^{(a)}\right)^{\prime \prime} \\
M_{3}^{\prime \prime} & =K_{14} U_{1}^{\prime \prime \prime}+K_{24} \phi^{\prime \prime \prime}-K_{34} U_{3}^{\prime \prime \prime \prime}+K_{44} U_{2}^{\prime \prime \prime \prime}-\left(M_{3}^{(a)}\right)^{\prime \prime}
\end{aligned}
$$

Sections 3.1.3 and 3.1.4 provide the expressions for the variation in kinetic energy 
and virtual work. These expressions are unchanged, but may be now combined with the variation in strain energy in order to form the linear equations of motion from solving Hamilton's principle. This describes the virtual displacements along the axial direction, lead-lag, flap and torsion directions. Furthermore it is known the boundary conditions due to kinetic energy, together with those from strain energy, reduce to zero,

$$
b(U)-b(T)=0
$$

Thus for contributions of strain energy, kinetic energy, and virtual work for axial displacements, one can separate the expressions to isolate $\delta u$ :

$$
\begin{aligned}
\text { from } \delta U: & -\int_{0}^{L} F_{1}^{\prime} \delta u d x \\
\text { from } \delta T: & \int_{0}^{L} \bar{Z}_{u} \delta u d x \\
& =\int_{0}^{L} m\left(\Omega^{2} x+2 \Omega \dot{v}\right) \delta u d x \\
\text { from } \delta W: & \int_{0}^{L} L_{u} \delta u d x \\
& =0
\end{aligned}
$$

which results in,

$$
\begin{aligned}
\int_{t_{1}}^{t_{2}}\left[\int_{0}^{L}\left(-F_{1}^{\prime}-m\left(\Omega^{2} x+2 \Omega \dot{v}\right)\right) \delta u d x-0\right] d t & =0 \\
-F_{1}^{\prime}-m \Omega^{2} x-2 m \Omega \dot{v} & =0
\end{aligned}
$$

Likewise, for the virtual displacements along the $y$-axis, $\delta v$, the expressions are:

$$
\begin{array}{ll}
\text { from } \delta U: & \int_{0}^{L}\left(M_{2}^{\prime \prime} \sin \theta+M_{3}^{\prime \prime} \cos \theta\right) \delta v d x \\
\text { from } \delta T: & \int_{0}^{L}\left(\bar{Z}_{v}-\bar{Z}_{v^{\prime}}^{\prime}\right) \delta v d x
\end{array}
$$




$$
\begin{aligned}
& =\int_{0}^{L}\left[m \Omega^{2} v+2 m \Omega\left(\beta_{p c} \dot{w}-\dot{u}\right)-m \ddot{v}\right] \delta v d x \\
\text { from } \delta W: \quad & \int_{0}^{L} L_{v} \delta v d x \\
& =\int_{0}^{L}\left(\frac { \rho _ { \infty } a c } { 2 } \left\{v_{i}^{2}-\Omega^{2} x^{2} \frac{c_{d}}{a}-\Omega x v_{i}(\theta+\phi)\right.\right. \\
& \left.\left.-\left(2 \Omega x \frac{c_{d}}{a}+(\theta+\phi) v_{i}\right) \dot{v}+\left(2 v_{i}-\Omega x(\theta+\phi)\right) \dot{w}\right\}\right) \delta v d x
\end{aligned}
$$

giving,

$$
\begin{aligned}
0 & =\int_{t_{1}}^{t_{2}}\left[\int_{0}^{L}\left(M_{2}^{\prime \prime} \sin \theta+M_{3}^{\prime \prime} \cos \theta-\left(m \Omega^{2} v+2 m \Omega\left(\beta_{p c} \dot{w}-\dot{u}\right)-m \ddot{v}\right)-L_{v}\right) \delta v d x\right] d t \\
L_{v} & =M_{2}^{\prime \prime} \sin \theta+M_{3}^{\prime \prime} \cos \theta-m \Omega^{2} v-2 m \Omega\left(\beta_{p c} \dot{w}-\dot{u}\right)+m \ddot{v}
\end{aligned}
$$

Strain, kinetic and aerodynamic energies for displacement along the $z$-axis give the expressions for $\delta w$ as:

$$
\begin{aligned}
\text { from } \delta U: & \int_{0}^{L}\left(M_{3}^{\prime \prime} \sin \theta-M_{2}^{\prime \prime} \cos \theta\right) \delta w d x \\
\text { from } \delta T: \quad & \int_{0}^{L}\left(\bar{Z}_{w}-\bar{Z}_{w^{\prime}}^{\prime}\right) \delta w d x \\
& =\int_{0}^{L}\left[-m \beta_{p c}\left(\Omega^{2} x+2 \Omega \dot{v}\right)-m \ddot{w}\right] \delta w d x \\
\text { from } \delta W: \quad & \int_{0}^{L} L_{w} \delta w d x \\
& =\int_{0}^{L}\left(\frac { \rho _ { \infty } a c } { 2 } \left\{-\Omega x v_{i}+\Omega^{2} x^{2}\left(\theta+\phi+\int_{0}^{x} v^{\prime} w^{\prime \prime} d x\right)\right.\right. \\
& -\Omega^{2} x v\left(\beta_{p c}+w^{\prime}\right)+\Omega^{2} \frac{x c}{2}\left(\beta_{p c}+w^{\prime}\right) \\
& \left.\left.+\left(2 \Omega x(\theta+\phi)-v_{i}\right) \dot{v}-\Omega x \dot{w}+\frac{3 c}{4} \Omega x \dot{\phi}-\frac{c}{4} \ddot{w}\right\}\right) \delta w d x
\end{aligned}
$$

to give,

$$
\begin{aligned}
0 & =\int_{t_{1}}^{t_{2}}\left[\int_{0}^{L}\left(M_{3}^{\prime \prime} \sin \theta-M_{2}^{\prime \prime} \cos \theta+m \beta_{p c}\left(\Omega^{2} x+2 \Omega \dot{v}\right)+m \ddot{w}-L_{w}\right) \delta w d x\right] d t \\
L_{w} & =M_{3}^{\prime \prime} \sin \theta-M_{2}^{\prime \prime} \cos \theta+m \beta_{p c}\left(\Omega^{2} x+2 \Omega \dot{v}\right)+m \ddot{w}
\end{aligned}
$$


Finally, virtual twist, $\delta \phi$, is given by the following equations:

$$
\begin{aligned}
\text { from } \delta U: & -\int_{0}^{L} M_{1}^{\prime} \delta \phi d x \\
\text { from } \delta T: & \int_{0}^{L} \bar{Z}_{\phi} \delta \phi d x \\
& =\int_{0}^{L}\left[-m k_{m} \ddot{\phi}-m \Omega^{2}\left(k_{m_{2}}^{2}-k_{m_{1}}^{2}\right) \cos (\theta+\phi) \sin (\theta+\phi)\right] \delta w d x \\
\text { from } \delta W: & \int_{0}^{L} M_{\phi} \delta \phi d x \\
& =\int_{0}^{L}\left(-\frac{\rho_{\infty} a c}{2}\left\{\frac{c^{2}}{8}\right)\right) \delta \phi d x
\end{aligned}
$$

giving,

$$
\begin{aligned}
0 & =\int_{t_{1}}^{t_{2}}\left[\int_{0}^{L}\left(-M_{1}^{\prime}+m k_{m} \ddot{\phi}-m \Omega^{2}\left(k_{m_{2}}^{2}-k_{m_{1}}^{2}\right) \cos (\theta+\phi) \sin (\theta+\phi)-M_{\phi}\right) \delta \phi d x\right] d t \\
M_{\phi} & =-M_{1}^{\prime}+m k_{m} \ddot{\phi}-m \Omega^{2}\left(k_{m_{2}}^{2}-k_{m_{1}}^{2}\right) \cos (\theta+\phi) \sin (\theta+\phi)
\end{aligned}
$$

The virtual displacement along the axial direction, $\delta u$, is redundant and it may be eliminated from the formulation. Substitution of the expressions in Eq. (C.8) into (C.9) and (C.10), will result in two expressions containing $u$. This variable is then expressed in terms of the other three by integrating $u^{\prime \prime}$ twice to get $u$, differentiating to obtain $\dot{u}$, and substituting into the relevant equations to get:

$$
\begin{aligned}
L_{v}= & {\left[\left(K_{33}-\frac{K_{13}^{2}}{K_{11}}\right) \sin ^{2} \theta+2\left(K_{34}-\frac{K_{13} K_{14}}{K_{11}}\right) \sin \theta \cos \theta\right.} \\
& \left.+\left(K_{44}-\frac{K_{14}^{2}}{K_{11}}\right) \cos ^{2} \theta\right] v^{\prime \prime \prime \prime} \\
& +\left[\left(K_{34}-\frac{K_{13} K_{14}}{K_{11}}\right) \sin ^{2} \theta+\left(K_{44}-K_{33}+\frac{K_{13}^{2}}{K_{11}}-\frac{K_{14}^{2}}{K_{11}}\right) \sin \theta \cos \theta\right. \\
& \left.-\left(K_{34}-\frac{K_{13} K_{14}}{K_{11}}\right) \cos ^{2} \theta\right] w^{\prime \prime \prime \prime} \\
& +\left[\left(K_{23}-\frac{K_{13} K_{12}}{K_{11}}\right) \sin \theta+\left(K_{24}-\frac{K_{14} K_{12}}{K_{11}}\right) \cos \theta\right] \phi^{\prime \prime \prime}
\end{aligned}
$$




$$
\begin{aligned}
& -m \Omega^{2} v-2 m \Omega\left(\beta_{p c} \dot{w}+\frac{K_{12}}{K_{11}} \dot{\phi}+\frac{\left(K_{13} \sin \theta+K_{14} \cos \theta\right)}{K_{11}} \dot{v}^{\prime}\right. \\
& \left.+\frac{\left(K_{14} \sin \theta-K_{13} \cos \theta\right)}{K_{11}} \dot{w}^{\prime}-\int_{0}^{x} \frac{\dot{F}_{1}^{(a)}}{K_{11}} d x\right)+m \ddot{v} \\
& +\frac{\left(K_{13} \sin \theta+K_{14} \cos \theta\right)}{K_{11}}\left(F_{1}^{(a)}\right)^{\prime}-\left(\left(M_{2}^{(a)}\right)^{\prime \prime} \sin \theta+\left(M_{3}^{(a)}\right)^{\prime \prime} \cos \theta\right) \\
& -\frac{\left(K_{13} \sin \theta+K_{14} \cos \theta\right)}{K_{11}} m\left(\Omega^{2}+2 \Omega \dot{v}^{\prime}\right)
\end{aligned}
$$

Similarly, for $L_{w}$ the resulting equation is:

$$
\begin{aligned}
L_{w}= & {\left[\left(K_{34}-\frac{K_{13} K_{14}}{K_{11}}\right) \sin ^{2} \theta+\left(K_{44}-K_{33}-\frac{K_{14}^{2}}{K_{11}}+\frac{K_{13}^{2}}{K_{11}}\right) \sin \theta \cos \theta\right.} \\
& \left.+\left(-K_{34}+\frac{K_{13} K_{14}}{K_{11}}\right) \cos ^{2} \theta\right] v^{\prime \prime \prime \prime} \\
& +\left[\left(K_{44}-\frac{K_{14}^{2}}{K_{11}}\right) \sin ^{2} \theta+\left(-2 K_{34}+2 \frac{K_{13} K_{14}}{K_{11}}\right) \sin \theta \cos \theta\right. \\
& \left.+\left(K_{33}-\frac{K_{13}^{2}}{K_{11}}\right) \cos ^{2} \theta\right] w^{\prime \prime \prime \prime} \\
& +\left[\left(K_{24}-\frac{K_{12} K_{14}}{K_{11}}\right) \sin \theta+\left(-K_{23}+\frac{K_{12} K_{13}}{K_{11}}\right) \cos \theta\right] \phi^{\prime \prime \prime} \\
& +\frac{\left(K_{14} \sin \theta-K_{13} \cos \theta\right)}{K_{11}}\left(F_{1}^{(a)}\right)^{\prime \prime}-\frac{\left(K_{14} \sin \theta-K_{13} \cos \theta\right)}{K_{11}} m\left(\Omega^{2}+2 \Omega \dot{v}\right) \\
& +\left(M_{2}^{(a)}\right)^{\prime \prime} \cos \theta-\left(M_{3}^{(a)}\right)^{\prime \prime} \sin \theta+m \beta_{p c}\left(\Omega^{2} x+2 \Omega \dot{v}\right)+m \ddot{w}
\end{aligned}
$$

and for $M_{\phi}$, the equation is:

$$
\begin{aligned}
M_{\phi}= & {\left[\left(-K_{23}+\frac{K_{12} K_{13}}{K_{11}}\right) \sin \theta+\left(-K_{24}+\frac{K_{12} K_{14}}{K_{11}}\right) \cos \theta\right] v^{\prime \prime \prime} } \\
& +\left[\left(-K_{24}+\frac{K_{12} K_{14}}{K_{11}}\right) \sin \theta+\left(K_{23}-\frac{K_{12} K_{13}}{K_{11}}\right) \cos \theta\right] w^{\prime \prime \prime} \\
& +\left[-K_{22}+\frac{K_{12}^{2}}{K_{11}}\right] \phi^{\prime \prime}-\frac{K_{12}}{K_{11}}\left(F_{1}^{(a)}\right)^{\prime}+\left(M_{1}^{(a)}\right)^{\prime} \\
& +m \frac{K_{12}}{K_{11}}\left(\Omega^{2} x+2 \Omega \dot{v}\right)+m k_{m} \ddot{\phi}+m \Omega^{2}\left(k_{m_{2}}^{2}-k_{m_{1}}^{2}\right) \cos (\theta+\phi) \sin (\theta+\phi)
\end{aligned}
$$


The above equations for $v, w$, and $\phi$, Eqs. (C.13) to (C.15), must be expressed nondimensionally. The following relationships define the nondimensional expressions for stiffness, displacements, mass radii of gyration, time, and derivatives:

$$
\begin{array}{llll}
\Lambda_{11}=\frac{K_{11}}{m \Omega^{2} L^{2}} & \Lambda_{23}=\frac{K_{23}}{m \Omega^{2} L^{4}} & \mu=\frac{k_{m}}{L} & \bar{v}=\frac{v}{L} \\
\Lambda_{12}=\frac{K_{12}}{m \Omega^{2} L^{3}} & \Lambda_{24}=\frac{K_{24}}{m \Omega^{2} L^{4}} & \mu_{1}=\frac{k_{m_{1}}}{L} & \bar{x}=\frac{w}{L} \\
\Lambda_{13}=\frac{K_{13}}{m \Omega^{2} L^{3}} & \Lambda_{33}=\frac{K_{33}}{m \Omega^{2} L^{4}} & \mu_{2}=\frac{k_{m_{2}}}{L} & \phi=\phi \\
\Lambda_{14}=\frac{K_{14}}{m \Omega^{2} L^{3}} & \Lambda_{34}=\frac{K_{34}}{m \Omega^{2} L^{4}} & \bar{c}=\frac{c}{L} & ()^{\prime}=\frac{d()}{d \bar{x}} \\
\Lambda_{22}=\frac{K_{22}}{m \Omega^{2} L^{4}} & \Lambda_{44}=\frac{K_{44}}{m \Omega^{2} L^{4}} & \bar{x}=\frac{x}{L} & \left(\dot{)}=\frac{1}{\Omega} \frac{d()}{d t}\right.
\end{array}
$$

Nondimensional forms for the actuation force and moments are:

$$
\begin{aligned}
\bar{F}_{1}^{(a)} & =\frac{F_{1}^{(a)}}{m \Omega^{2} L^{2}} \\
\bar{M}_{1}^{(a)} & =\frac{M_{1}^{(a)}}{m \Omega^{2} L^{3}} \\
\bar{M}_{2}^{(a)} & =\frac{M_{2}^{(a)}}{m \Omega^{2} L^{3}} \\
\bar{M}_{3}^{(a)} & =\frac{M_{3}^{(a)}}{m \Omega^{2} L^{3}}
\end{aligned}
$$

From Eq. (C.13), the resulting nondimensional expression for $\bar{v}$ is:

$$
\begin{aligned}
L_{\bar{v}}= & {\left[\left(\Lambda_{33}-\frac{\Lambda_{13}^{2}}{\Lambda_{11}}\right) \sin ^{2} \theta+\left(\Lambda_{34}-\frac{\Lambda_{13} \Lambda_{14}}{\Lambda_{11}}\right) \sin 2 \theta+\left(\Lambda_{44}-\frac{\Lambda_{14}^{2}}{\Lambda_{11}}\right) \cos ^{2} \theta\right] \bar{v}^{\prime \prime \prime \prime}(\text { C.16 }) } \\
& +\left[-\left(\Lambda_{34}-\frac{\Lambda_{13} \Lambda_{14}}{\Lambda_{11}}\right) \cos 2 \theta+\left(\Lambda_{44}-\Lambda_{33}+\frac{\Lambda_{13}^{2}}{\Lambda_{11}}-\frac{\Lambda_{14}^{2}}{\Lambda_{11}}\right) \frac{\sin 2 \theta}{2}\right] \bar{w}^{\prime \prime \prime \prime} \\
& +\left[\left(\Lambda_{23}-\frac{\Lambda_{13} \Lambda_{12}}{\Lambda_{11}}\right) \sin \theta+\left(\Lambda_{24}-\frac{\Lambda_{14} \Lambda_{12}}{\Lambda_{11}}\right) \cos \theta\right] \phi^{\prime \prime \prime} \\
& +\frac{\left(\Lambda_{13} \sin \theta+\Lambda_{14} \cos \theta\right)}{\Lambda_{11}}\left(\left(\bar{F}_{1}^{(a)}\right)^{\prime \prime}-1-4 \dot{\bar{v}}^{\prime}\right)-\frac{\left(\Lambda_{14} \sin \theta-\Lambda_{13} \cos \theta\right)}{\Lambda_{11}}\left(2 \dot{\bar{w}}^{\prime}\right) \\
& -2\left(\beta_{p c} \dot{\bar{w}}+\frac{\Lambda_{12}}{\Lambda_{11}} \dot{\phi}-\int_{0}^{\bar{x}} \frac{\dot{\bar{F}}_{1}^{(a)}}{\Lambda_{11}} d \bar{x}\right)+\ddot{\bar{v}}-\bar{v}-\left(\bar{M}_{2}^{(a)}\right)^{\prime \prime} \sin \theta-\left(\bar{M}_{3}^{(a)}\right)^{\prime \prime} \cos \theta
\end{aligned}
$$


From Eq. (C.14), the resulting nondimensional expression for $\bar{w}$ is:

$$
\begin{aligned}
L_{\bar{w}}= & {\left[-\left(\Lambda_{34}-\frac{\Lambda_{13} \Lambda_{14}}{\Lambda_{11}}\right) \cos 2 \theta+\left(\Lambda_{44}-\frac{\Lambda_{14}^{2}}{\Lambda_{11}}-\Lambda_{33}+\frac{\Lambda_{13}^{2}}{\Lambda_{11}}\right) \frac{\sin 2 \theta}{2}\right] \bar{v}^{\prime \prime \prime \prime} } \\
& +\left[\left(\Lambda_{44}-\frac{\Lambda_{14}^{2}}{\Lambda_{11}}\right) \sin ^{2} \theta-\left(\Lambda_{34}-\frac{\Lambda_{13} \Lambda_{14}}{\Lambda_{11}}\right) \sin 2 \theta+\left(\Lambda_{33}-\frac{\Lambda_{13}^{2}}{\Lambda_{11}}\right) \cos ^{2} \theta\right] \bar{w}^{\prime \prime \prime \prime} \\
& +\left[\left(\Lambda_{24}-\frac{\Lambda_{12} \Lambda_{14}}{\Lambda_{11}}\right) \sin \theta-\left(\Lambda_{23}-\frac{\Lambda_{12} \Lambda_{13}}{\Lambda_{11}}\right) \cos \theta\right] \phi^{\prime \prime \prime} \\
& +\frac{\left(\Lambda_{14} \sin \theta-\Lambda_{13} \cos \theta\right)}{\Lambda_{11}}\left(\left(\bar{F}_{1}^{(a)}\right)^{\prime \prime}-1+2 \dot{\bar{v}}^{\prime}\right) \\
& +\left(\bar{M}_{2}^{(a)}\right)^{\prime \prime} \cos \theta-\left(\bar{M}_{3}^{(a)}\right)^{\prime \prime} \sin \theta+\beta_{p c} \bar{x}+2 \beta_{p c} \dot{\bar{v}}+m \ddot{\bar{w}}
\end{aligned}
$$

From Eq. (C.15), the resulting nondimensional expression for $\phi$ is:

$$
\begin{aligned}
M_{\phi}= & {\left[-\left(\Lambda_{23}-\frac{\Lambda_{12} \Lambda_{13}}{\Lambda_{11}}\right) \sin \theta-\left(\Lambda_{24}-\frac{\Lambda_{12} \Lambda_{14}}{\Lambda_{11}}\right) \cos \theta\right] \bar{v}^{\prime \prime \prime} } \\
& +\left[-\left(\Lambda_{24}-\frac{\Lambda_{12} \Lambda_{14}}{\Lambda_{11}}\right) \sin \theta+\left(\Lambda_{23}-\frac{\Lambda_{12} \Lambda_{13}}{\Lambda_{11}}\right) \cos \theta\right] \bar{w}^{\prime \prime \prime} \\
& +\left[-\Lambda_{22}+\frac{\Lambda_{12}^{2}}{\Lambda_{11}}\right] \phi^{\prime \prime}-\frac{\Lambda_{12}}{\Lambda_{11}}\left(-\bar{x}-2 \dot{\bar{v}}+\left(\bar{F}_{1}^{(a)}\right)^{\prime}\right)+\left(\bar{M}_{1}^{(a)}\right)^{\prime} \\
& +\mu^{2} \ddot{\phi}+\left(\mu_{2}^{2}-\mu_{1}^{2}\right)\left(\phi \cos 2 \theta+\frac{\sin 2 \theta}{2}\right)
\end{aligned}
$$

The addition of the aerodynamic terms to Eqs. (C.16) to (C.18) completes the expressions for displacements in lead-lag, flap, and torsion. The above equations may be further reduced by defining the following variables to replace some of the repeated expressions:

$$
\begin{aligned}
& \Gamma_{1}=\frac{\Lambda_{13} \sin \theta+\Lambda_{14} \cos \theta}{\Lambda_{11}} \\
& \Gamma_{2}=\frac{\Lambda_{14} \sin \theta-\Lambda_{13} \cos \theta}{\Lambda_{11}} \\
& \Gamma_{3}=\Lambda_{23}-\frac{\Lambda_{12} \Lambda_{13}}{\Lambda_{11}} \\
& \Gamma_{4}=\Lambda_{24}-\frac{\Lambda_{12} \Lambda_{14}}{\Lambda_{11}}
\end{aligned}
$$




$$
\begin{aligned}
& \Gamma_{5}=\Lambda_{34}-\frac{\Lambda_{13} \Lambda_{14}}{\Lambda_{11}} \\
& \Gamma_{6}=\Lambda_{33}-\frac{\Lambda_{13}^{2}}{\Lambda_{11}} \\
& \Gamma_{7}=\Lambda_{33}-\frac{\Lambda_{13}^{2}}{\Lambda_{11}}
\end{aligned}
$$

The final expressions for the nondimensional equations of motion are,

$$
\begin{aligned}
& L_{\bar{v}}=\left(\Gamma_{5} \sin 2 \theta+\Gamma_{6} \sin ^{2} \theta+\Gamma_{7} \cos ^{2} \theta\right) \bar{v}^{\prime \prime \prime \prime} \\
& +\left(-\Gamma_{5} \cos 2 \theta+\left(\Gamma_{7}-\Gamma_{6}\right) \frac{\sin 2 \theta}{2}\right) \bar{w}^{\prime \prime \prime \prime} \\
& +\left(\Gamma_{3} \sin \theta+\Gamma_{4} \cos \theta\right) \phi^{\prime \prime \prime}+\Gamma_{1}\left(\left(\bar{F}_{1}^{(a)}\right)^{\prime \prime}-1-4 \dot{\bar{v}}^{\prime}\right)-\Gamma_{2}\left(2 \dot{\bar{w}}^{\prime}\right) \\
& -2\left(\beta_{p c} \dot{\bar{w}}+\frac{\Lambda_{12}}{\Lambda_{11}} \dot{\phi}-\int_{0}^{\bar{x}} \frac{\dot{\bar{F}}^{(a)}}{\Lambda_{11}} d \bar{x}\right)+\ddot{\bar{v}}-\bar{v}-\left(\bar{M}_{2}^{(a)}\right)^{\prime \prime} \sin \theta-\left(\bar{M}_{3}^{(a)}\right)^{\prime \prime} \cos \theta \\
& =\frac{\gamma}{6}\left[\bar{v}_{i}^{2}-\bar{x}^{2} \frac{c_{d}}{a}-\bar{x} \bar{v}_{i} \theta\right]-\frac{\gamma}{6}\left[\bar{x} \bar{v}_{i} \phi+\left[2 \bar{x} \frac{c_{d}}{a}+(\theta+\phi) \bar{v}_{i}\right] \dot{\bar{v}}-\left[2 \bar{v}_{i}-\bar{x}(\theta+\phi)\right] \dot{\bar{w}}\right] \\
& L_{\bar{w}}=\left[-\Gamma_{5} \cos 2 \theta+\left(\Gamma_{7}-\Gamma_{6}\right) \frac{\sin 2 \theta}{2}\right] \bar{v}^{\prime \prime \prime} \\
& +\left(-\Gamma_{5} \sin 2 \theta+\Gamma_{6} \cos ^{2} \theta+\Gamma_{7} \sin ^{2} \theta\right) \bar{w}^{\prime \prime \prime \prime} \\
& +\left(-\Gamma_{3} \cos \theta+\Gamma_{4} \sin \theta\right) \phi^{\prime \prime \prime}+\Gamma_{2}\left(\left(\bar{F}_{1}^{(a)}\right)^{\prime \prime}-1+2 \dot{\bar{v}}^{\prime}\right)+\beta_{p c} \bar{x}+2 \beta_{p c} \dot{\bar{v}}+\ddot{\bar{w}} \\
& -\left(\bar{M}_{3}^{(a)}\right)^{\prime \prime} \sin \theta+\left(\bar{M}_{2}^{(a)}\right)^{\prime \prime} \cos \theta \\
& =\frac{\gamma}{6}\left[-\bar{x} \bar{v}_{i}+\bar{x}^{2} \theta+\frac{\bar{c}}{2} \bar{x} \beta_{p c}\right]-\frac{\gamma}{6}\left[\bar{x}^{2}\left(\phi+\int_{0}^{\bar{x}} \bar{v}^{\prime} \bar{w}^{\prime \prime} d \bar{x}\right)+\bar{x} \bar{v}\left(\beta_{p c}+\bar{w}^{\prime}\right)-\frac{\bar{c}}{2} \bar{x} \bar{w}^{\prime}\right. \\
& \left.-\left[2 \bar{x}(\theta+\phi)-\bar{v}_{i}\right] \dot{\bar{v}}+\bar{x} \dot{\bar{w}}-\frac{3 \bar{c}}{4} \bar{x} \dot{\phi}\right]-\frac{\gamma}{6} \frac{\bar{c}}{4} \ddot{\bar{w}} \\
& M_{\phi}=\left(-\Gamma_{3} \sin \theta-\Gamma_{4} \cos \theta\right) \bar{v}^{\prime \prime \prime}+\left(\Gamma_{3} \cos \theta-\Gamma_{4} \sin \theta\right) \bar{w}^{\prime \prime \prime}+\left(\frac{\Lambda_{12}^{2}}{\Lambda_{11}}-\Lambda_{22}\right) \phi^{\prime \prime} \\
& -\frac{\Lambda_{12}}{\Lambda_{11}}\left(-\bar{x}-2 \dot{\bar{v}}+\left(\bar{F}_{1}^{(a)}\right)^{\prime}\right)+\mu^{2} \ddot{\phi}+\left(\bar{M}_{1}^{(a)}\right)^{\prime}+\left(\mu_{2}^{2}-\mu_{1}^{2}\right)\left(\phi \cos 2 \theta+\frac{\sin 2 \theta}{2}\right) \\
& =-\frac{\gamma}{6}\left(\frac{\bar{c}^{2}}{8} \bar{x} \dot{\phi}\right)
\end{aligned}
$$


A solution for these complex multi-variable equations of motion is best determined by applying an assumed modes method to the equations. Galerkin's method is one such solution. The dimensionless bending and torsion displacements are replaced by the expression of Eq. (3.34). These expressions are composed of a modal generalized coordinate $-V_{j}, W_{j}$, and $\Phi_{j}$ - and a mode shape function - $\Psi_{j}$ or $\Theta_{j}$.

For the lead-lag displacements, $\bar{v}$, Eq. (C.19) results in an expression in terms of these modal functions:

$$
\begin{aligned}
& \sum_{j=1}^{N}\left\langle\delta _ { i j } \left[\left\{\left(\Gamma_{5} \sin 2 \theta+\Gamma_{6} \sin ^{2} \theta+\Gamma_{7} \cos ^{2} \theta\right) \beta_{j}^{4}-1\right\} V_{j}\right.\right. \\
& \left.+\left\{-\Gamma_{5} \cos 2 \theta+\left(\Gamma_{7}-\Gamma_{6}\right) \frac{\sin 2 \theta}{2}\right\} \beta_{j}^{4} W_{j}-2 \beta_{p c} \dot{W}_{j}+\ddot{V}_{j}\right] \\
& +\left[-Q_{i j} \Phi_{j}\left\{\Gamma_{3} \sin \theta+\Gamma_{4} \cos \theta\right\}\right]-\left[2 N_{i j}\left\{2 \Gamma_{1} \dot{V}_{j}+\Gamma_{2} \dot{W}_{j}\right\}\right] \\
& +\left[\frac{2}{\Lambda_{11}}\left\{\dot{E}_{e} F_{i j}^{(a)}-\Lambda_{12} D_{i j} \dot{\Phi}_{j}\right\}\right]+E_{e}\left[\Gamma_{1} A_{i j}^{(a)}-C_{i j}^{(a)} \sin \theta-D_{i j}^{(a)} \cos \theta\right] \\
& +\frac{\gamma}{6}\left[\bar{v}_{i} I_{i j} \Phi_{j}+\left(2 \frac{c_{d}}{a} E_{i j}+\theta \bar{v}_{i} \delta_{i j}+\bar{v}_{i} \sum_{k=1}^{N} \Phi_{k} H_{i j k}\right) \dot{V}_{j}\right. \\
& \left.\left.-\left(2 \bar{v}_{i} \delta_{i j}-\theta E_{i j}-\sum_{k=1}^{N} \Phi_{k} G_{i j k}\right) \dot{W}_{j}\right]\right\rangle \\
& =\Gamma_{1} A_{i}+\frac{\gamma}{6}\left(\bar{v}_{i}^{2} A_{i}-\frac{c_{d}}{a} C_{i}-\bar{v}_{i} \theta B_{i}\right)
\end{aligned}
$$

For the flap displacements, $\vec{w}$, Eq. (C.20) becomes:

$$
\begin{aligned}
& \sum_{j=1}^{N}\left\langle\delta _ { i j } \left[\left\{-\Gamma_{5} \cos 2 \theta+\left(\Gamma_{7}-\Gamma_{6}\right) \frac{\sin 2 \theta}{2}\right\} \beta_{j}^{4} V_{j}\right.\right. \\
& +\left\{-\Gamma_{5} \sin 2 \theta+\Gamma_{6} \cos ^{2} \theta+\Gamma_{7} \sin ^{2} \theta\right\} \beta_{j}^{4} W_{j} \\
& \left.+2 \beta_{p c} \dot{V}_{j}+\left(1+\frac{\gamma \bar{c}}{24}\right) \ddot{W}_{j}\right] \\
& +\left[P_{i j} \Phi_{j}\left\{-\Gamma_{3} \cos \theta+\Gamma_{4} \sin \theta\right\}\right]+\left[2 \Gamma_{2} N_{i j} \dot{V}_{j}\right] \\
& +\left[E_{e}\left\{\Gamma_{2} A_{i j}^{(a)}-D_{i j}^{(a)} \sin \theta+C_{i j}^{(a)} \cos \theta\right\}\right]
\end{aligned}
$$




$$
\begin{aligned}
& +\frac{\gamma}{6}\left[-J_{i j} \Phi_{j}+\sum_{k=1}^{N} L_{i j k} V_{j} W_{j}+\beta_{p c} E_{i j} V_{j}-\frac{\bar{c}}{2} O_{i j} W_{j}\right. \\
& \left.\left.-\left(2 \theta E_{i j}+2 \sum_{k=1}^{N} \Phi_{k} G_{i j k}-\bar{v}_{i} \delta_{i j}\right) \dot{V}_{j}+E_{i j} \dot{W}_{j}-\frac{3 \bar{c}}{4} I_{i j} \dot{\Phi}_{j}\right]\right) \\
& =\frac{\gamma}{6}\left(-\bar{v}_{i} B_{i}+\theta C_{i}+\frac{\bar{c}}{2} \beta_{p c} B_{i}\right)-\beta_{p c} B_{i}+\Gamma_{2} A_{i}
\end{aligned}
$$

For torsional displacements, $\phi$, Eq. (C.21) becomes:

$$
\begin{aligned}
& \sum_{j=1}^{N}\left\{Q_{i j}\left[\left\{-\Gamma_{3} \sin \theta-\Gamma_{4} \cos \theta\right\} V_{j}+\left\{\Gamma_{3} \cos \theta-\Gamma_{4} \sin \theta\right\} W_{j}\right]\right. \\
& +\delta_{i j}\left[\Phi_{j}\left\{\left(\mu_{2}^{2}-\mu_{1}^{2}\right) \cos 2 \theta-\left(\frac{\Lambda_{12}^{2}}{\Lambda_{11}}-\Lambda_{22}\right) \gamma_{j}^{2}\right\}+\ddot{\Phi}_{j} \mu^{2}\right] \\
& +\left[2 \frac{\Lambda_{12}}{\Lambda_{11}} D_{i j} \dot{V}_{j}\right]+\left[E_{e}\left\{-\frac{\Lambda_{12}}{\Lambda_{11}} E_{i j}^{(a)}-B_{i j}^{(a)}\right\}\right] \\
& \left.+\left[\frac{\gamma}{48} \dot{c}^{2} M_{i j} \dot{\Phi}_{j}\right]\right\rangle \\
& =-\frac{\sin 2 \theta}{\sqrt{2} \gamma_{j}}\left(\mu_{2}^{2}-\mu_{1}^{2}\right)-\frac{\Lambda_{12}}{\Lambda_{11}} F_{i}
\end{aligned}
$$

where new modal integrals contained in Eqs. (C.22) to (C.24), that either add to or change some of the expression of Hodges and Ormiston are,

$$
\begin{aligned}
D_{i j} & =\int_{0}^{1} \Psi_{i} \Theta_{j} d \bar{x} \\
F_{i} & =\int_{0}^{1} \bar{x} \Theta_{i} d \bar{x} \\
K_{i j} & =n u l l \\
N_{i j} & =\int_{0}^{1} \Psi_{i} \Psi_{j}^{\prime} d \bar{x} \\
P_{i j} & =\int_{0}^{1} \Psi_{i} \Theta_{j}^{\prime \prime \prime} d \bar{x} \\
Q_{i j} & =\int_{0}^{1} \Psi_{i}^{\prime \prime \prime} \Theta_{j} d \bar{x}
\end{aligned}
$$


and the active modal integrals are:

$$
\begin{aligned}
A_{i j}^{(a)} & =\int_{0}^{1} \Psi_{i}^{\prime \prime} \bar{F}_{1 j}^{(a)} d \bar{x} \\
B_{i j}^{(a)} & =\int_{0}^{1} \Theta_{i}^{\prime} \bar{M}_{1 j}^{(a)} d \bar{x} \\
C_{i j}^{(a)} & =\int_{0}^{1} \Psi_{i}^{\prime \prime} \bar{M}_{2 j}^{(a)} d \bar{x} \\
D_{i j}^{(a)} & =\int_{0}^{1} \Psi_{i}^{\prime \prime} \bar{M}_{3 j}^{(a)} d \bar{x} \\
E_{i j}^{(a)} & =\int_{0}^{1} \Theta_{i}^{\prime} \bar{F}_{1 j}^{(a)} d \bar{x}
\end{aligned}
$$

Steady-state and perturbation equations may subsequently be carried out. This completes the summary of a linear analysis for the equations of motion for an active helicopter rotor blade in hover. The equations, variables, and modal integrals should be considered separate to those of Chapter 3 , and do not contribute to the analytical beam formulation that is applied in the remainder of the thesis. 


\section{Appendix D}

\section{Cross-Sectional Properties}

The following describe the cross-sectional properties for the thin-walled blade cross-section as outlined out by Hodges and Ormiston,

$$
\begin{aligned}
A & =\iint_{A} d \eta d \zeta & A e_{A} & =\iint_{A} \eta d \eta d \zeta \\
I_{y^{\prime}} & =\iint_{A} \zeta^{2} d \eta d \zeta & I_{z^{\prime}} & =\iint_{A} \eta^{2} d \eta d \zeta \\
A k_{A}^{2} & =\iint_{A}\left(\eta^{2}+\zeta^{2}\right) d \eta d \zeta & J & =\iint_{A}\left(\hat{\eta}^{2}+\hat{\zeta}^{2}\right) d \eta d \zeta \\
B_{1}^{\star} & =\iint_{A}\left(\eta^{2}+\zeta^{2}\right)^{2} d \eta d \zeta & B_{2}^{\star} & =\iint_{A} \eta\left(\eta^{2}+\zeta^{2}\right) d \eta d \zeta \\
C_{1} & =\iint_{A} \lambda^{2} d \eta d \zeta & C_{1}^{\star} & =\iint_{A} \zeta \lambda d \eta d \zeta
\end{aligned}
$$

where $A$ denotes the blade cross-sectional area effective in carrying tension, $e_{A}$ is the tension offset from the elastic axis, $I_{y}$ and $I_{z}$ are the moments of inertia in the flapwise and chordwise directions, $J$ is the torsional constant, $A k_{A}$ is the polar moment of inertia, $C_{1}$ is warping rigidity, and $B_{1}^{\star}, B_{2}^{\star}$, and $C_{1}^{\star}$ are section constants.

A symmetric blade cross-section about the $\eta$ axis signifies that terms containing a product with $\zeta$ go to zero. This is not the case for an asymmetric cross-section. However because of the antisymmetric character of a section undergoing deformation with warping, those expressions containing products of the warping term, $\lambda$, are set to zero. 
The section integrals as defined by the terms of the kinetic energy equation of Section 3.1.3 are,

$$
\begin{aligned}
m & =\iint_{A} \rho d \eta d \zeta & k_{m}^{2} & =k_{m_{1}}^{2}+k_{m_{2}}^{2} \\
k_{m_{1}}^{2} & =\frac{1}{m} \iint_{m} \rho \zeta^{2} d \eta d \zeta & k_{m_{2}}^{2} & =\frac{1}{m} \iint_{m} \rho \eta^{2} d \eta d \zeta
\end{aligned}
$$

where $m$ is the mass per unit length, $k_{m}$ represents the blade cross-section mass radius of gyration, and $k_{m_{1}}$ and $k_{m_{2}}$ represent the principal mass radii of gyration. 


\section{Appendix E}

\section{Actuation Vector Transformation}

In order to incorporate the actuation vector from the cross-sectional analysis into the strain energy equation of the $1 \mathrm{D}$ beam formulation, the components of the actuation vector in the deformed frame need to be determined.

For example, in reference to Figure 3.3 the actuated moments $M^{(a)}$ may be written as

$$
M^{(a)}=M_{1}^{(a)} \vec{i}+M_{2}^{(a)} \vec{j}+M_{3}^{(a)} \vec{k} \equiv M_{x}^{(a)} \vec{i}+M_{y}^{(a)} \vec{j}+M_{z}^{(a)} \vec{k}
$$

in the undeformed frame. These equations may be expressed in the deformed frame as,

$$
M^{(a)}=M_{x^{\prime}}^{(a)} \overrightarrow{i^{\prime}}+M_{y^{\prime}}^{(a)} \overrightarrow{j^{\prime}}+M_{z^{\prime}}^{(a)} \overrightarrow{k^{\prime}}
$$

Thus,

$$
M^{(a)}=M_{x^{\prime}}^{(a)} \overrightarrow{i^{\prime}}+M_{y^{\prime}}^{(a)} \overrightarrow{j^{\prime}}+M_{z^{\prime}}^{(a)} \overrightarrow{k^{\prime}}=M_{x}^{(a)} \vec{i}+M_{y}^{(a)} \vec{j}+M_{z}^{(a)} \vec{k}
$$

Multiplying through by $\overrightarrow{i^{\prime}}$ to isolate $M_{x^{\prime}}^{(\alpha)}$,

$$
M_{x^{\prime}}^{(a)} \overrightarrow{i^{\prime}} \cdot \overrightarrow{i^{\prime}}+M_{y^{\prime}}^{(a)} \overrightarrow{j^{\prime}} \cdot \overrightarrow{i^{\prime}}+M_{z^{\prime}}^{(a)} \overrightarrow{k^{\prime}} \cdot \overrightarrow{i^{\prime}}=M_{x}^{(a)} \vec{i} \cdot \overrightarrow{i^{\prime}}+M_{y}^{(a)} \vec{j} \cdot \overrightarrow{i^{\prime}}+M_{z}^{(a)} \vec{k} \cdot \overrightarrow{i^{\prime}}
$$




$$
\begin{aligned}
M_{x^{\prime}}^{(a)}= & M_{x}^{(a)} \vec{i} \cdot\left[\vec{i}\left(1-\frac{1}{2} v^{\prime 2}-\frac{1}{2} w^{2}\right)+v^{\prime} \vec{j}+w^{\prime} \vec{k}\right] \\
& +M_{y}^{(a)} \vec{j} \cdot\left[\vec{i}\left(1-\frac{1}{2} v^{\prime 2}-\frac{1}{2} w^{2}\right)+v^{\prime} \vec{j}+w^{\prime} \vec{k}\right] \\
& +M_{z}^{(a)} \vec{k} \cdot\left[\vec{i}\left(1-\frac{1}{2} v^{2}-\frac{1}{2} w^{\prime 2}\right)+v^{\prime} \vec{j}+w^{\prime} \vec{k}\right] \\
= & M_{x}^{(a)}\left[\left(1-\frac{1}{2} v^{\prime 2}-\frac{1}{2} w^{\prime 2}\right)\right]+M_{y}^{(a)} v^{\prime}+M_{z}^{(a)} w^{\prime}
\end{aligned}
$$

and multiplying through by $\overrightarrow{j^{\prime}}$ to isolate $M_{y^{\prime}}^{(a)}$,

$$
\begin{aligned}
M_{x^{\prime}}^{(a)} \overrightarrow{\vec{i}^{\prime}} \cdot \overrightarrow{j^{\prime}}+M_{y^{\prime}}^{(a)} \overrightarrow{j^{\prime}} \cdot \overrightarrow{j^{\prime}}+M_{z^{\prime}}^{(a)} \overrightarrow{k^{\prime}} \cdot \overrightarrow{j^{\prime}}= & M_{x}^{(a)} \vec{i} \cdot \overrightarrow{j^{\prime}}+M_{y}^{(a)} \vec{j} \cdot \overrightarrow{j^{\prime}}+M_{z}^{(a)} \vec{k} \cdot \overrightarrow{j^{\prime}} \\
M_{y^{\prime}}^{(a)}= & M_{x}^{(a)} \vec{i} \cdot\left\{\left[-v^{\prime} \cos (\theta+\phi)-w^{\prime} \sin (\theta+\phi)\right] \vec{i}\right\} \\
& +M_{x}^{(a)} \vec{i} \cdot\left\{\left(1-\frac{1}{2} v^{\prime 2}\right) \cos \left(\theta+\hat{\phi}+v^{\prime} w^{\prime}\right) \vec{j}\right\} \\
& +M_{x}^{(a)} \vec{i} \cdot\left\{\left(1-\frac{1}{2} w^{\prime 2}\right) \sin (\theta+\hat{\phi}) \vec{k}\right\} \\
& +M_{y}^{(a)} \vec{j} \cdot\{[\ldots] \vec{i}+[\ldots] \vec{j}+[\ldots] \vec{k}\} \\
& +M_{z}^{(a)} \vec{k} \cdot\{[\ldots] \vec{i}+[\ldots] \vec{j}+[\ldots] \vec{k}\} \\
= & -M_{x}^{(a)}\left[v^{\prime} \cos (\theta+\phi)+w^{\prime} \sin (\theta+\phi)\right] \\
& +M_{y}^{(a)}\left(1-\frac{1}{2} v^{2}\right) \cos \left(\theta+\hat{\phi}+v^{\prime} w^{\prime}\right) \\
& +M_{z}^{(a)}\left(1-\frac{1}{2} w^{\prime 2}\right) \sin (\theta+\hat{\phi})
\end{aligned}
$$

and finally, multiplying through by $\overrightarrow{k^{\prime}}$ to isolate $M_{z^{\prime}}^{(a)}$,

$$
\begin{aligned}
M_{x^{\prime}}^{(a)} \overrightarrow{i^{\prime}} \cdot \overrightarrow{k^{\prime}}+M_{y^{\prime}}^{(a)} \overrightarrow{j^{\prime}} \cdot \overrightarrow{k^{\prime}}+M_{z^{\prime}}^{(a)} \overrightarrow{k^{\prime}} \cdot \overrightarrow{k^{\prime}}= & M_{x}^{(a)} \vec{i} \cdot \overrightarrow{k^{\prime}}+M_{y}^{(a)} \vec{j} \cdot \overrightarrow{k^{\prime}}+M_{z}^{(a)} \vec{k} \cdot \overrightarrow{k^{\prime}} \\
M_{z^{\prime}}^{(a)}= & M_{x}^{(a)} \vec{i} \cdot\{[\ldots] \vec{i}+[\ldots] \vec{j}+[\ldots] \vec{k}\} \\
& +M_{y}^{(a)} \vec{j} \cdot\{[\ldots] \vec{i}+[\ldots] \vec{j}+[\ldots] \vec{k}\} \\
& +M_{z}^{(a)} \vec{k} \cdot\{[\ldots] \vec{i}+[\ldots] \vec{j}+[\ldots] \vec{k}\} \\
= & M_{x}^{(a)}\left[v^{\prime} \sin (\theta+\phi)-w^{\prime} \cos (\theta+\phi)\right]
\end{aligned}
$$




$$
\begin{aligned}
& -M_{y}^{(a)}\left(1-\frac{1}{2} v^{2}\right) \sin \left(\theta+\hat{\phi}+v^{\prime} w^{\prime}\right) \\
& +M_{z}^{(a)}\left(1-\frac{1}{2} w^{\prime 2}\right) \cos (\theta+\hat{\phi})
\end{aligned}
$$

Therefore, in accordance with the ordering scheme used by Hodges and Ormiston, the actuated moment vectors may be reduced, by elimination of higher order terms, to

$$
\begin{aligned}
M_{x^{\prime}}^{(a)}= & M_{x}^{(a)}+v^{\prime} M_{y}^{(a)}+w^{\prime} M_{z}^{(a)} \\
M_{y^{\prime}}^{(a)}= & -M_{x}^{(a)}\left[v^{\prime} \cos (\theta+\phi)+w^{\prime} \sin (\theta+\phi)\right]+M_{y}^{(a)} \cos (\theta+\phi) \\
& +M_{z}^{(a)} \sin (\theta+\phi) \\
M_{z^{\prime}}^{(a)}= & M_{x}^{(a)}\left[v^{\prime} \sin (\theta+\phi)-w^{\prime} \cos (\theta+\phi)\right]-M_{y}^{(a)} \sin (\theta+\phi) \\
& +M_{z}^{(a)} \cos (\theta+\phi)
\end{aligned}
$$

Likewise, $F^{(a)}$ may be found in the same fashion, however the final terms contain only those in the axial direction and so it reduces to

$$
\begin{aligned}
F_{x^{\prime}}^{(a)} & =F_{x}^{(a)}+v^{\prime} F_{y}^{(a)}+w^{\prime} F_{z}^{(a)} \\
& =F_{x}^{(a)}
\end{aligned}
$$




\section{Appendix F}

\section{Nondimensional Constants and}

\section{Galerkin Integrals}

\section{F.1 Nondimensional Constants}

The dimensionless parameters given in Chapter 3 are

$$
\begin{array}{rlrl}
K & =\frac{k_{A}^{2}}{k_{m}^{2}} & \kappa & =\frac{G J}{m \Omega^{2} L^{4}} \\
\mu_{1} & =\frac{k_{m_{1}}}{L} & \gamma & =\frac{3 \rho_{\infty} a c L}{m} \\
\mu_{2} & =\frac{k_{m_{2}}}{L} & \bar{v} & =\frac{v}{L} \\
\mu & =\frac{k_{m}}{L} & \bar{w} & =\frac{w}{L} \\
\bar{c} & =\frac{c}{L} & \bar{v} & =\frac{v_{i}}{\Omega L} \\
\bar{x} & =\frac{x}{L} & ()^{\prime} & =\frac{\partial}{\partial \bar{x}}() \\
\Lambda_{1} & =\frac{E I_{y^{\prime}}}{m \Omega^{2} L^{4}} & \left.()^{\prime}\right) & =(\cdot)=\frac{\partial}{\partial \psi}=\frac{\partial}{\Omega \partial t}() \\
\Lambda_{2} & =\frac{E I_{z^{\prime}}}{m \Omega^{2} L^{4}} &
\end{array}
$$


The dimensionless actuation parameters are

$$
\begin{array}{ll}
\bar{F}_{x}^{(a)}=\frac{F_{x}^{(a)}}{m \Omega^{2} L^{2}} & \bar{M}_{x}^{(a)}=\frac{M_{x}^{(a)}}{m \Omega^{2} L^{3}} \\
\bar{M}_{y}^{(a)}=\frac{M_{y}^{(a)}}{m \Omega^{2} L^{3}} & \bar{M}_{z}^{(a)}=\frac{M_{z}^{(a)}}{m \Omega^{2} L^{3}}
\end{array}
$$

\section{F.2 Galerkin Integrals}

The non-active Galerkin integrals are as follows

$$
\begin{array}{rlrl}
A_{i} & =\int_{0}^{1} \Psi_{i} d \bar{x} & J_{i j} & =\int_{0}^{1} \bar{x}^{2} \Psi_{i} \Theta_{j} d \bar{x} \\
B_{i} & =\int_{0}^{1} \bar{x} \Psi_{i} d \bar{x} & K_{i j k} & =\int_{0}^{1} \Theta_{i} \Psi_{j}^{\prime \prime} \Psi_{k}^{\prime \prime} d \bar{x} \\
C_{i} & =\int_{0}^{1} \bar{x}^{2} \Psi_{i} d \bar{x} & L_{i j k} & =\int_{0}^{1} \bar{x} \Psi_{i} \Psi_{j} \Psi_{k}^{\prime} d \bar{x} \\
D_{i j}=\int_{0}^{1}\left(\frac{1-\bar{x}^{2}}{2}\right) \Psi_{i}^{\prime} \Psi_{j}^{\prime} d \bar{x} & & & -\int_{0}^{1} \bar{x}^{2} \Psi_{i} \int_{0}^{\bar{x}} \Psi_{j}^{\prime}\left(\overline{x_{1}}\right) \Psi_{k}^{\prime \prime}\left(\overline{x_{1}}\right) d \overline{x_{1}} d \bar{x} \\
E_{i j}=\int_{0}^{1} \bar{x} \Psi_{i} \Psi_{j} d \bar{x} & M_{i j} & =\int_{0}^{1} \bar{x} \Theta_{i} \Theta_{j} d \bar{x} \\
F_{i j k}=-\frac{1}{\beta_{k}^{4}} \int_{0}^{1} \bar{x} \Psi_{i}^{\prime} \Psi_{j}^{\prime} \Psi_{k}^{\prime \prime \prime} d \bar{x} & N_{i j} & =\int_{0}^{1}\left(\frac{1-\bar{x}^{2}}{2}\right) \Theta_{i}^{\prime} \Theta_{j}^{\prime} d \bar{x} \\
G_{i j k}=\int_{0}^{1} \bar{x} \Psi_{i} \Psi_{j} \Theta_{k} d \bar{x} & O_{i j} & =\int_{0}^{1} \bar{x} \Psi_{i} \Psi_{j}^{\prime} d \bar{x} \\
H_{i j k}=\int_{0}^{1} \Psi_{i} \Psi_{j} \Theta_{k} d \bar{x} & \delta_{i j} & =\int_{0}^{1} \Psi_{i} \Psi_{j} d \bar{x}=\int_{0}^{1} \Theta_{i} \Theta_{j} d \bar{x} \\
I_{i j}=\int_{0}^{1} \bar{x} \Psi_{i} \Theta_{j} d \bar{x} & & =0 i \neq j \\
& & & =1 i=j
\end{array}
$$

The active Galerkin integrals are

$$
\begin{aligned}
A_{i j}^{(a)}=\int_{0}^{1} \Psi_{i} \mathbf{F}_{x j}^{(a)} \bar{x} d \bar{x} & F_{i j}^{(a)}=\int_{0}^{1} \Psi_{i}^{\prime \prime} \mathbf{M}_{y j}^{(a)} d \bar{x} \\
B_{i j k}^{(a)}=\int_{0}^{1} \Psi_{i}^{\prime \prime} \Psi_{k}^{\prime} \mathbf{M}_{x j}^{(a)} d \bar{x} & G_{i j k}^{(a)}=\int_{0}^{1} \Theta_{i}^{\prime} \Psi_{k}^{\prime} \mathbf{M}_{y j}^{(a)} d \bar{x} \\
C_{i j}^{(a)}=\int_{0}^{1} \Theta_{i}^{\prime} \mathbf{M}_{x j}^{(a)} d \bar{x} & H_{i j k}^{(a)}=\int_{0}^{1} \Theta_{i} \Psi_{k}^{\prime \prime} \mathbf{M}_{z j}^{(a)} d \bar{x} \\
D_{i j k}^{(a)}=\int_{0}^{1} \Theta_{i} \Psi_{k}^{\prime \prime} \mathbf{M}_{y j}^{(a)} d \bar{x} & I_{i j k}^{(a)}=\int_{0}^{1} \Theta_{i}^{\prime} \Psi_{k}^{\prime} \mathbf{M}_{z j}^{(a)} d \bar{x} \\
E_{i j}^{(a)}=\int_{0}^{1} \Psi_{i}^{\prime \prime} \mathbf{M}_{z j}^{(a)} d \bar{x} & J_{i j k l}^{(a)}=\int_{0}^{1} \Theta_{i} \Psi_{k}^{\prime} \Psi_{l}^{\prime \prime} \mathbf{M}_{x j}^{(a)} d \bar{x}
\end{aligned}
$$




\section{F.3 Segmented Actuated Galerkin Integrals}

$$
\begin{aligned}
A_{i j}^{(a)} & =\int_{0}^{x_{1}} \Psi_{i} \mathbf{F}_{x j}^{(a)} x d \bar{x}+\int_{x_{1}}^{x_{2}} \Psi_{i} \mathbf{F}_{x j}^{(a)} x d \bar{x}+\int_{x_{2}}^{1} \Psi_{i} \mathbf{F}_{x j}^{(a)} x d \bar{x} \\
B_{i j k}^{(a)} & =\int_{0}^{x_{1}} \Psi_{i}^{\prime \prime} \Psi_{k}^{\prime} \mathbf{M}_{x j}^{(a)} d \bar{x}+\int_{x_{1}}^{x_{2}} \Psi_{i}^{\prime \prime} \Psi_{k}^{\prime} \mathbf{M}_{x j}^{(a)} d \bar{x}+\int_{x_{2}}^{1} \Psi_{i}^{\prime \prime} \Psi_{k}^{\prime} \mathbf{M}_{x j}^{(a)} d \bar{x} \\
C_{i j}^{(a)} & =\int_{0}^{x_{1}} \Theta_{i}^{\prime} \mathbf{M}_{x j}^{(a)} d \bar{x}+\int_{x_{1}}^{x_{2}} \Theta_{i}^{\prime} \mathbf{M}_{x j}^{(a)} d \bar{x}+\int_{x_{2}}^{1} \Theta_{i}^{\prime} \mathbf{M}_{x j}^{(a)} d \bar{x} \\
D_{i j k}^{(a)} & =\int_{0}^{x_{1}} \Theta_{i} \Psi_{k}^{\prime \prime} \mathbf{M}_{y j}^{(a)} d \bar{x}+\int_{x_{1}}^{x_{2}} \Theta_{i} \Psi_{k}^{\prime \prime} \mathbf{M}_{y j}^{(a)} d \bar{x}+\int_{x_{2}}^{1} \Theta_{i} \Psi_{k}^{\prime \prime} \mathbf{M}_{y j}^{(a)} d \bar{x} \\
E_{i j}^{(a)} & =\int_{0}^{x_{1}} \Psi_{i}^{\prime \prime} \mathbf{M}_{z j}^{(a)} d \bar{x}+\int_{x_{1}}^{x_{2}} \Psi_{i}^{\prime \prime} \mathbf{M}_{z j}^{(a)} d \bar{x}+\int_{x_{2}}^{1} \Psi_{i}^{\prime \prime} \mathbf{M}_{z j}^{(a)} d \bar{x} \\
F_{i j}^{(a)} & =\int_{0}^{x_{1}} \Psi_{i}^{\prime \prime} \mathbf{M}_{y j}^{(a)} d \bar{x}+\int_{x_{1}}^{x_{2}} \Psi_{i}^{\prime \prime} \mathbf{M}_{y j}^{(a)} d \bar{x}+\int_{x_{2}}^{1} \Psi_{i}^{\prime \prime} \mathbf{M}_{y j}^{(a)} d \bar{x} \\
G_{i j k}^{(a)} & =\int_{0}^{x_{1}} \Theta_{i}^{\prime} \Psi_{k}^{\prime} \mathbf{M}_{y j}^{(a)} d \bar{x}+\int_{x_{1}}^{x_{2}} \Theta_{i}^{\prime} \Psi_{k}^{\prime} \mathbf{M}_{y j}^{(a)} d \bar{x}+\int_{x_{2}}^{1} \Theta_{i}^{\prime} \Psi_{k}^{\prime} \mathbf{M}_{y j}^{(a)} d \bar{x} \\
H_{i j k}^{(a)} & =\int_{0}^{x_{1}} \Theta_{i} \Psi_{k}^{\prime \prime} \mathbf{M}_{z j}^{(a)} d \bar{x}+\int_{x_{1}}^{x_{2}} \Theta_{i} \Psi_{k}^{\prime \prime} \mathbf{M}_{z j}^{(a)} d \bar{x}+\int_{x_{2}}^{1} \Theta_{i} \Psi_{k}^{\prime \prime} \mathbf{M}_{z j}^{(a)} d \bar{x} \\
I_{i j k}^{(a)} & =\int_{0}^{x_{1}} \Theta_{i}^{\prime} \Psi_{k}^{\prime} \mathbf{M}_{z j}^{(a)} d \bar{x}+\int_{x_{1}}^{x_{2}} \Theta_{i}^{\prime} \Psi_{k}^{\prime} \mathbf{M}_{z j}^{(a)} d \bar{x}+\int_{x_{2}}^{1} \Theta_{i}^{\prime} \Psi_{k}^{\prime} \mathbf{M}_{z j}^{(a)} d \bar{x} \\
J_{i j k l}^{(a)} & =\int_{0}^{x_{1}} \Theta_{i} \Psi_{k}^{\prime} \Psi_{l}^{\prime \prime} \mathbf{M}_{x j}^{(a)} d \bar{x}+\int_{x_{1}}^{x_{2}} \Theta_{i} \Psi_{k}^{\prime} \Psi_{l}^{\prime \prime} \mathbf{M}_{x j}^{(a)} d \bar{x}+\int_{x_{2}}^{1} \Theta_{i} \Psi_{k}^{\prime} \Psi_{l}^{\prime \prime} \mathbf{M}_{x j}^{(a)} d \bar{x}
\end{aligned}
$$

\section{F.4 Actuation from $x_{1}$ to $x_{2}$}

$$
\begin{aligned}
A_{i j}^{(a)} & =\int_{x_{1}}^{x_{2}} \Psi_{i}(\bar{x}) \frac{\Theta_{j}^{\prime}(\bar{x})}{\sum_{k=1}^{N} \Theta_{k}^{\prime}(\bar{x})} \bar{F}_{x}^{(a)} x d \bar{x} \\
B_{i j k}^{(a)} & =\int_{x_{1}}^{x_{2}} \Psi_{i}^{\prime \prime}(\bar{x}) \Psi_{k}^{\prime}(\bar{x}) \frac{\Theta_{j}^{\prime}(\bar{x})}{\sum_{k=1}^{N} \Theta_{k}^{\prime}(\bar{x})} \bar{M}_{x}^{(a)} d \bar{x} \\
C_{i j}^{(a)} & =\int_{x_{1}}^{x_{2}} \Theta_{i}^{\prime}(\bar{x}) \frac{\Theta_{j}^{\prime}(\bar{x})}{\sum_{k=1}^{N} \Theta_{k}^{\prime}(\bar{x})} \bar{M}_{x}^{(a)} d \bar{x} \\
D_{i j k}^{(a)} & =\int_{x_{1}}^{x_{2}} \Theta_{i}(\bar{x}) \Psi_{k}^{\prime \prime}(\bar{x}) \frac{\Psi_{j}^{\prime \prime}(\bar{x})}{\sum_{k=1}^{N} \Psi_{k}^{\prime \prime}(\bar{x})} \bar{M}_{y}^{(a)} d \bar{x} \\
E_{i j}^{(a)} & =\int_{x_{1}}^{x_{2}} \Psi_{i}^{\prime \prime}(\bar{x}) \frac{\Psi_{j}^{\prime \prime}(\bar{x})}{\sum_{k=1}^{N} \Psi_{k}^{\prime \prime}(\bar{x})} \bar{M}_{z}^{(a)} d \bar{x} \\
F_{i j}^{(a)} & =\int_{x_{1}}^{x_{2}} \Psi_{i}^{\prime \prime}(\bar{x}) \frac{\Psi_{j}^{\prime \prime}(\bar{x})}{\sum_{k=1}^{N} \Psi_{k}^{\prime \prime}(\bar{x})} \bar{M}_{y}^{(a)} d \bar{x}
\end{aligned}
$$


APPENDIX F. NONDIMENSIONAL CONSTANTS AND GALERKIN INTEGRALS155

$$
\begin{aligned}
G_{i j k}^{(a)} & =\int_{x_{1}}^{x_{2}} \Theta_{i}^{\prime}(\bar{x}) \Psi_{k}^{\prime}(\bar{x}) \frac{\Psi_{j}^{\prime \prime}(\bar{x})}{\sum_{k=1}^{N} \Psi_{k}^{\prime \prime}(\bar{x})} \bar{M}_{y}{ }^{(a)} d \bar{x} \\
H_{i j k}^{(a)} & =\int_{x_{1}}^{x_{2}} \Theta_{i}(\bar{x}) \Psi_{k}^{\prime \prime}(\bar{x}) \frac{\Psi_{j}^{\prime \prime}(\bar{x})}{\sum_{k=1}^{N} \Psi_{k}^{\prime \prime}(\bar{x})} \bar{M}_{z}^{(a)} d \bar{x} \\
I_{i j k}^{(a)} & =\int_{x_{1}}^{x_{2}} \Theta_{i}^{\prime}(\bar{x}) \Psi_{k}^{\prime}(\bar{x}) \frac{\Psi_{j}^{\prime \prime}(\bar{x})}{\sum_{k=1}^{N} \Psi_{k}^{\prime \prime}(\bar{x})} \bar{M}_{z}^{(a)} d \bar{x} \\
J_{i j k l}^{(a)} & =\int_{x_{1}}^{x_{2}} \Theta_{i}(\bar{x}) \Psi_{k}^{\prime}(\bar{x}) \Psi_{l}^{\prime \prime}(\bar{x}) \frac{\Theta_{j}^{\prime}(\bar{x})}{\sum_{k=1}^{N} \Theta_{k}^{\prime}(\bar{x} x)} \bar{M}_{x}{ }^{(a)} d \bar{x}
\end{aligned}
$$




\section{Appendix G}

\section{Perturbation Equations}

The mass matrix of the dynamic equation for the active blade is

$$
[M]=\left[\begin{array}{ccc}
\delta_{i j} & 0 & 0 \\
0 & \delta_{i j}\left(1+\frac{\gamma \bar{c}}{24}\right) & 0 \\
0 & 0 & \mu^{2} \delta_{i j}
\end{array}\right]
$$

The damping matrices are

$$
\begin{aligned}
& {[C]=2\left[\begin{array}{ccc}
\sum_{k=1}^{N}\left(F_{i k j}-F_{j k i}\right) V_{0 k} & -\beta_{p c} \delta_{i j}-\sum_{k=1}^{N} F_{j k i} W_{0 k} & 0 \\
\beta_{p c} \delta_{i j}+\sum_{k=1}^{N} F_{i k j} W_{0 k} & 0 & 0 \\
0 & 0 & 0
\end{array}\right]} \\
& +\frac{\gamma}{6}\left[\begin{array}{ccc}
2 \frac{c_{j}}{a} E_{i j}+\theta \bar{v}_{i} \delta_{i j}+\bar{v}_{i} \sum_{k=1}^{N} H_{i j k} \Phi_{0 k} & -\left(2 \bar{v}_{i} \delta_{i j}-\theta E_{i j}-\sum_{k=1}^{N} G_{i j k} \Phi_{0 k}\right) & 0 \\
-2 \theta E_{i j}+\bar{v}_{i} \delta_{i j}-2 \sum_{k=1}^{N} G_{i j k} \Phi_{0 k} & E_{i j} & -\frac{3 \bar{c}}{4} I_{i j} \\
0 & 0 & \frac{\bar{c}^{2}}{8} M_{i j}
\end{array}\right]
\end{aligned}
$$

and the stiffness matrices are

$$
[K]=\left[\begin{array}{lll}
K_{11}^{s} & K_{12}^{s} & K_{13}^{s} \\
K_{21}^{s} & K_{22}^{s} & K_{23}^{s} \\
K_{31}^{s} & K_{32}^{s} & K_{33}^{s}
\end{array}\right]+\frac{\gamma}{6}\left[\begin{array}{ccc}
K_{11}^{a} & K_{12}^{a} & K_{13}^{a} \\
K_{21}^{a} & K_{22}^{a} & K_{23}^{a} \\
K_{31}^{a} & K_{32}^{a} & K_{33}^{a}
\end{array}\right]
$$


with $s$ and $a$ representing the structural (with actuation) part and the aerodynamic part.

These values for $K_{i j}$ are

$$
\begin{aligned}
& K_{11}^{s}=D_{i j}+\left[\Lambda_{2}-\left(\Lambda_{2}-\Lambda_{1}\right) \sin ^{2} \theta\right] \beta_{j}^{4} \delta_{i j}-\delta_{i j} \\
& -\left(\Lambda_{2}-\Lambda_{1}\right) \sum_{k=1}^{N} K_{k j i} \Phi_{0 k} \sin (2 \theta) \\
& K_{12}^{s}=\frac{\sin (2 \theta)}{2}\left(\Lambda_{2}-\Lambda_{1}\right) \beta_{j}^{4} \delta_{i j} \\
& +\left(\Lambda_{2}-\Lambda_{1}\right) \sum_{k=1}^{N}\left(K_{k j i} \Phi_{0 k} \cos (2 \theta)+B_{i j k}^{(a)}\right) \\
& K_{13}^{s}=\left(\Lambda_{2}-\Lambda_{1}\right) \sum_{k=1}^{N} K_{j k i}\left[-V_{0 k} \sin (2 \theta)+W_{0 k} \cos (2 \theta)\right] \\
& K_{21}^{s}=\frac{\sin (2 \theta)}{2}\left(\Lambda_{2}-\Lambda_{1}\right) \beta_{j}^{4} \delta_{i j} \\
& +\left(\Lambda_{2}-\Lambda_{1}\right) \sum_{k=1}^{N}\left(K_{k j i} \Phi_{0 k} \cos (2 \theta)-B_{i j k}^{(a)}\right) \\
& K_{22}^{s}=D_{i j}+\left[\Lambda_{1}+\left(\Lambda_{2}-\Lambda_{1}\right) \sin ^{2} \theta\right] \beta_{j}^{4} \delta_{i j} \\
& +\left(\Lambda_{2}-\Lambda_{1}\right) \sum_{k=1}^{N} K_{k j i} \Phi_{0 k} \sin (2 \theta) \\
& K_{23}^{s}=\left(\Lambda_{2}-\Lambda_{1}\right) \sum_{k=1}^{N} K_{j k i}\left[V_{0 k} \cos (2 \theta)+W_{0 k} \sin (2 \theta)\right] \\
& K_{31}^{s}=\left(\Lambda_{2}-\Lambda_{1}\right) \sum_{k=1}^{N} K_{i j k}\left[-V_{0 k} \sin (2 \theta)+W_{0 k} \cos (2 \theta)\right] \\
& +\sum_{k=1}^{N}\left[-\left(G_{i j k}^{(a)}+D_{i j k}^{(a)}\right)+\sum_{l=1}^{N} 2 J_{i j k l}^{(a)} V_{0 l}\right] \\
& K_{32}^{s}=\left(\Lambda_{2}-\Lambda_{1}\right) \sum_{k=1}^{N} K_{i j k}\left[V_{0 k} \cos (2 \theta)+W_{0 k} \sin (2 \theta)\right] \\
& +\sum_{k=1}^{N}\left[-\left(I_{i j k}^{(a)}+H_{i j k}^{(a)}\right)+\sum_{l=1}^{N} 2 J_{i j k l}^{(a)} W_{0 l}\right] \\
& K_{33}^{s}=\mu^{2} K N_{i j}+\left[\kappa \gamma_{j}^{2}+\left(\mu_{2}^{2}-\mu_{1}^{2}\right) \cos (2 \theta)\right] \delta_{i j}
\end{aligned}
$$


and

$$
\begin{aligned}
& K_{11}^{a}=0 \\
& K_{12}^{a}=0 \\
& K_{13}^{a}=\bar{v}_{i} I_{i j} \\
& K_{21}^{a}=\beta_{p c} E_{i j}+\sum_{k=1}^{N} L_{i j k} W_{0 k} \\
& K_{22}^{a}=-\frac{\bar{c}}{2} O_{i j}+\sum_{k=1}^{N} L_{i k j} V_{0 k} \\
& K_{23}^{a}=-J_{i j} \\
& K_{31}^{a}=0 \\
& K_{32}^{a}=0 \\
& K_{33}^{a}=0
\end{aligned}
$$

The actuation vector, $\{F\}$ is:

$$
\{F\}=\left\{\begin{array}{c}
-E_{i j}^{(a)} \\
F_{i j}^{(a)} \\
-C_{i j}^{(a)}
\end{array}\right\}
$$




\section{Appendix $\mathbf{H}$}

\section{Box Beam Variables}

The $y$ and $z$ coordinates of a box beam may be represented in terms of $s$ as

\begin{tabular}{llll} 
side & $s$ & $y(s)$ & $z(s)$ \\
\hline top left & $0 \rightarrow q$ & $-s+\frac{q-r}{2}$ & $\frac{p}{2}$ \\
left vertical & $0 \rightarrow p$ & $-\frac{q+r}{2}$ & $\frac{p}{2}-s$ \\
bottom left & $0 \rightarrow q$ & $s-\frac{(q+r)}{2}$ & $-\frac{p}{2}$ \\
web & $0 \rightarrow p$ & $\frac{q-r}{2}$ & $-\frac{p}{2}+s / \frac{p}{2}-s$ \\
bottom right & $0 \rightarrow r$ & $\frac{q-r}{2}+s$ & $-\frac{p}{2}$ \\
right vertical & $0 \rightarrow p$ & $\frac{q+r}{2}$ & $-\frac{p}{2}+s$ \\
top right & $0 \rightarrow r$ & $\frac{q+r}{2}-s$ & $\frac{p}{2}$
\end{tabular}

Integral expressions for the stiffness variables of a box beam are reduced to:

$$
\begin{aligned}
\int_{I} \frac{B}{C} d s & =q \frac{B_{t}}{C_{t}}+p \frac{B_{l v}}{C_{l v}}+q \frac{B_{b}}{C_{b}}+p \frac{B_{w}}{C_{w}} \\
\int_{I} \frac{B}{C} y d s & =-\frac{r q}{2} \frac{B_{t}}{C_{t}}-\frac{p}{2}(q+r) \frac{B_{l v}}{C_{l v}}-\frac{r q}{2} \frac{B_{b}}{C_{b}}+\frac{p}{2}(q-r) \frac{B_{w}}{C_{w}} \\
\int_{I I} \frac{B}{C} d s & =p \frac{B_{w}}{C_{w}}+r \frac{B_{b}}{C_{b}}+p \frac{B_{r v}}{C_{r v}}+r \frac{B_{t}}{C_{t}} \\
\int_{I I} \frac{B}{C} y d s & =\frac{p}{2}(q-r) \frac{B_{w}}{C_{w}}+\frac{r q}{2} \frac{B_{b}}{C_{b}}+\frac{p}{2}(q+r) \frac{B_{r v}}{C_{r v}}+\frac{r q}{2} \frac{B_{t}}{C_{t}}
\end{aligned}
$$




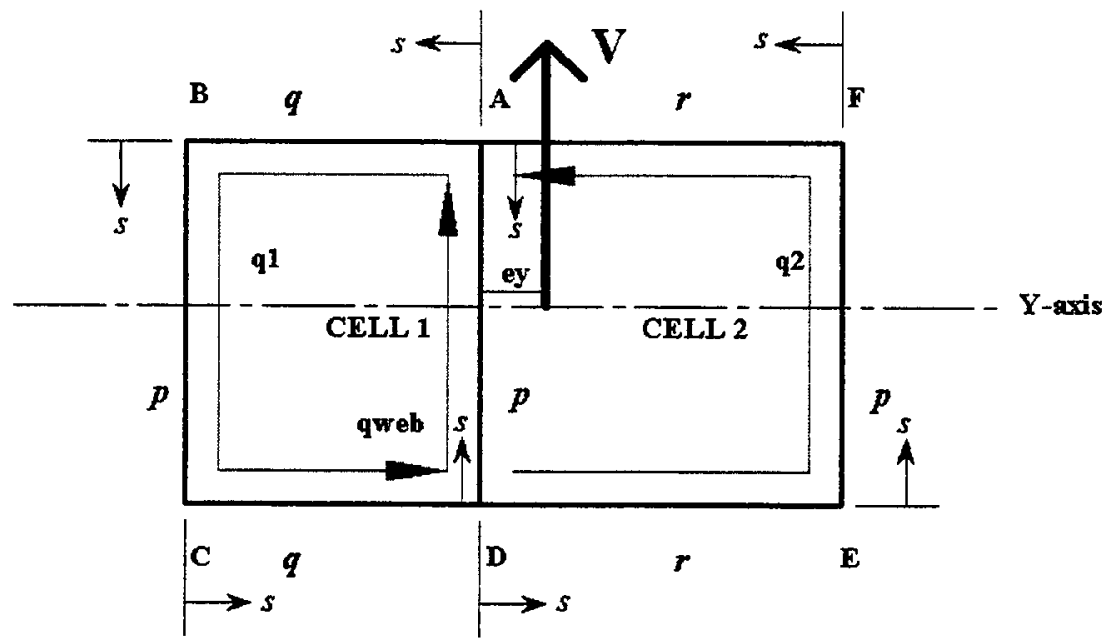

Figure H.1: Box beam coordinates and shear flow parameters

$$
\begin{aligned}
& \int_{I} \frac{B}{C} z d s=\frac{q p}{2} \frac{B_{t l}}{C_{t l}}-\frac{q p}{2} \frac{B_{b l}}{C_{b l}} \\
& \int_{I I} \frac{B}{C} z d s=-\frac{r p}{2} \frac{B_{b r}}{C_{b r}}+\frac{r p}{2} \frac{B_{t r}}{C_{t r}} \\
& \int_{a l l}\left(A-\frac{B^{2}}{C}\right) d s=\left(A_{t}-\frac{B_{t}^{2}}{C_{t}}\right)(q+r)+\left(A_{l v}-\frac{B_{l v}^{2}}{C_{l v}}\right) p+\left(A_{b}-\frac{B_{b}^{2}}{C_{b}}\right)(q+r) \\
& +\left(A_{w}-\frac{B_{w}^{2}}{C_{w}}\right) p+\left(A_{r v}-\frac{B_{r v}^{2}}{C_{r v}}\right) p \\
& \int_{a l l}\left(A-\frac{B^{2}}{C}\right) y d s=-\left(A_{l v}-\frac{B_{l v}^{2}}{C_{l v}}\right) \frac{p}{2}(q+r)+\left(A_{w}-\frac{B_{w}^{2}}{C_{w}}\right) \frac{p}{2}(q-r) \\
& +\left(A_{r v}-\frac{B_{r v}^{2}}{C_{r v}}\right) \frac{p}{2}(q+r) \\
& \int_{\text {all }}\left(A-\frac{B^{2}}{C}\right) z d s=\left(A_{t}-\frac{B_{t}^{2}}{C_{t}}\right) \frac{p}{2}(q+r)-\left(A_{b}-\frac{B_{b}^{2}}{C_{b}}\right) \frac{p}{2}(q+r) \\
& \int_{a l l}\left(A-\frac{B^{2}}{C}\right) y^{2} d s=\left(A_{t}-\frac{B_{t}^{2}}{C_{t}}\right)\left(\frac{q^{3}+r^{3}}{12}+\frac{q r^{2}+q^{2} r}{4}\right)+\left(A_{l v}-\frac{B_{l v}^{2}}{C_{l v}}\right) \frac{p}{4}(q+r)^{2} \\
& +\left(A_{b}-\frac{B_{b}^{2}}{C_{b}}\right)\left(\frac{q^{3}+r^{3}}{12}+\frac{q r^{2}+q^{2} r}{4}\right)+\left(A_{w}-\frac{B_{w}^{2}}{C_{w}}\right) \frac{p}{4}(q-r)^{2} \\
& +\left(A_{r v}-\frac{B_{r v}^{2}}{C_{r v}}\right) \frac{p}{4}(q+r)^{2} \\
& \int_{a l l}\left(A-\frac{B^{2}}{C}\right) z^{2} d s=\left(A_{t}-\frac{B_{t}^{2}}{C_{t}}\right) \frac{p^{2}}{4}(q+r)+\left(A_{l v}-\frac{B_{l v}^{2}}{C_{l v}}\right) \frac{p^{3}}{12}
\end{aligned}
$$




$$
\begin{aligned}
& +\left(A_{b}-\frac{B_{b}^{2}}{C_{b}}\right) \frac{p^{2}}{4}(q+r)+\left(A_{w}-\frac{B_{w}^{2}}{C_{w}}\right) \frac{p^{3}}{12} \\
& +\left(A_{r v}-\frac{B_{r v}^{2}}{C_{r v}}\right) \frac{p^{3}}{12} \\
\int_{\text {all }}\left(A-\frac{B^{2}}{C}\right) y z d s= & 0
\end{aligned}
$$

where $t, b, l v, r v$, w signify the top, bottom, left vertical, right vertical and web respectively. This also assumes that:

$$
A_{b r}=A_{b l} ; \quad B_{b r}=B_{b l} ; \quad C_{b r}=C_{b l}
$$

and

$$
A_{t r}=A_{t l} ; \quad B_{t r}=B_{t l} ; \quad C_{t r}=C_{t l}
$$

The $d$ variables become:

$$
\begin{aligned}
& d_{1}=\int_{I} y b(s) d s+\int_{I I} y b(s) d s=p(q+r) \frac{B_{l v}}{C_{l v}}-2 p(q-r) \frac{B_{w}}{C_{w}}-p(q+r) \frac{B_{r v}}{C_{r v}} \\
& d_{2}=\int_{I} z b(s) d s+\int_{I I} z b(s) d s=-p(q+r) \frac{B_{t}}{C_{t}}+p(q+r) \frac{B_{b}}{C_{b}}
\end{aligned}
$$

The $b$ variables become:

$$
\begin{aligned}
& b_{1}=4 \int_{0}^{s_{1}} c(s) d s=-8\left(\frac{q}{C_{t}}+\frac{p}{C_{l v}}+\frac{q}{C_{b}}\right) \\
& b_{2}=4 \int_{s_{1}}^{s_{2}} c(s) d s=-8 \frac{p}{C_{w}} \\
& b_{3}=\int_{I} b(s) d s=-2\left(q \frac{B_{t}}{C_{t}}+p \frac{B_{l v}}{C_{l v}}+q \frac{B_{b}}{C_{b}}+p \frac{B_{w}}{C_{w}}\right) \\
& b_{4}=-\int_{I} r_{n} d s=-2 q p \\
& b_{5}=4 \int_{s_{2}}^{s_{3}} c(s) d s=-8\left(\frac{r}{C_{b}}+\frac{p}{C_{r v}}+\frac{r}{C_{t}}\right) \\
& b_{6}=\int_{I I} b(s) d s=-2\left(p \frac{B_{w}}{C_{w}}+r \frac{B_{b}}{C_{b}}+p \frac{B_{r v}}{C_{r v}}+r \frac{B_{t}}{C_{t}}\right) \\
& b_{7}=-\int_{I} r_{n} d s=-2 r p
\end{aligned}
$$


APPENDIX H. BOX BEAM VARIABLES

$$
\begin{aligned}
A e_{I} & =q p \\
A e_{I I} & =r p
\end{aligned}
$$




\section{Appendix I}

\section{Trim Deflections}

The following tables and figures provide the stiffness and equilibrium deflection information for Designs 1 and 7 .

Table I.1: Stiffness values for Designs 1 and 7

\begin{tabular}{|l|c|c|c|c|c|c|}
\hline & \multicolumn{3}{|c|}{ Design 1 } & \multicolumn{3}{c|}{ Design 7 } \\
\hline & Baseline & 2 active plies & 4 active plies & Baseline & 2 active plies & 4 active plies \\
\hline$K_{11}(\mathrm{~N})$ & 9458976.0 & 9347769.8 & 9192307.5 & 10778384.4 & 10844101.2 & 9744251.8 \\
$K_{12}(\mathrm{Nm})$ & 0.0 & 0.0 & 0.0 & 15320.8 & 12594.2 & 4456.4 \\
$K_{13}(\mathrm{Nm})$ & -398.5 & -660.2 & -885.1 & -190.0 & -420.6 & -834.0 \\
$K_{14}(\mathrm{Nm})$ & -27454.8 & -42754.4 & -55883.1 & -15526.2 & -28554.1 & -51551.4 \\
$K_{22}\left(\mathrm{Nm}^{2}\right)$ & 221.9 & 186.8 & 140.9 & 221.4 & 224.0 & 200.7 \\
$K_{23}\left(\mathrm{Nm}^{2}\right)$ & 0.0 & 0.0 & 0.0 & 3.4 & 2.4 & 0.5 \\
$K_{24}\left(\mathrm{Nm}^{2}\right)$ & 0.0 & 0.0 & 0.0 & 180.9 & 126.1 & 23.2 \\
$K_{33}\left(\mathrm{Nm}^{2}\right)$ & 312.3 & 307.9 & 301.7 & 319.8 & 337.3 & 318.9 \\
$K_{34}\left(\mathrm{Nm}^{2}\right)$ & 141.5 & 142.8 & 144.3 & 144.5 & 146.1 & 144.9 \\
$K_{44}\left(\mathrm{Nm}^{2}\right)$ & 9696.3 & 9745.5 & 9794.9 & 9832.2 & 10052.3 & 9866.1 \\
\hline$F_{x}^{(a)}$ & - & 56.5 & 25.3 & - & -296.1 & -812.8 \\
$M_{x}^{(a)}$ & - & -2.9 & -3.6 & - & -2.0 & -4.2 \\
$M_{y}^{(a)}$ & - & 0.0 & 0.0 & - & -0.1 & -0.2 \\
$M_{z}^{(a)}$ & - & 0.8 & 0.3 & - & -3.4 & -8.6 \\
\hline
\end{tabular}




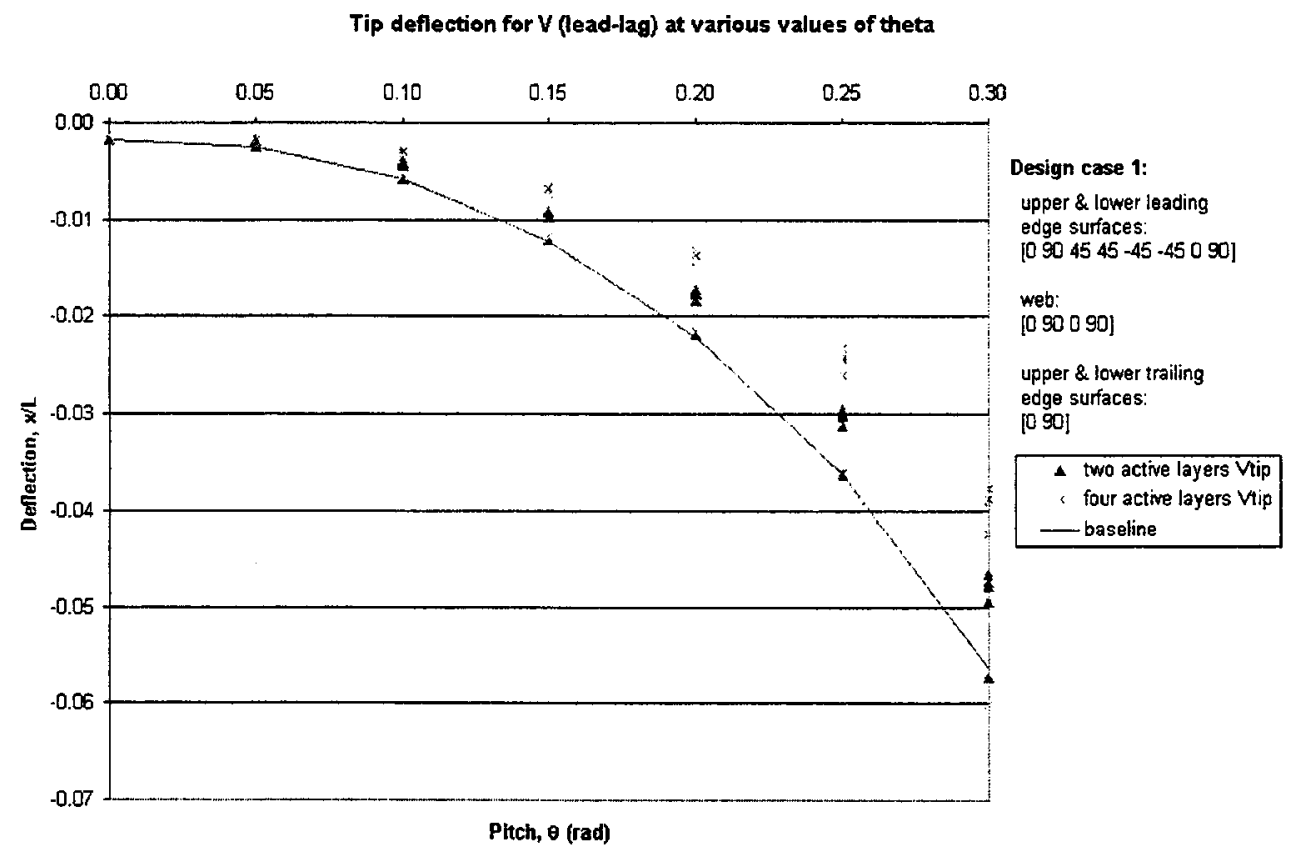

Figure I.1: Equilibrium tip deflections in lead-lag bending

Tip deflection for W (flap) at various values of theta

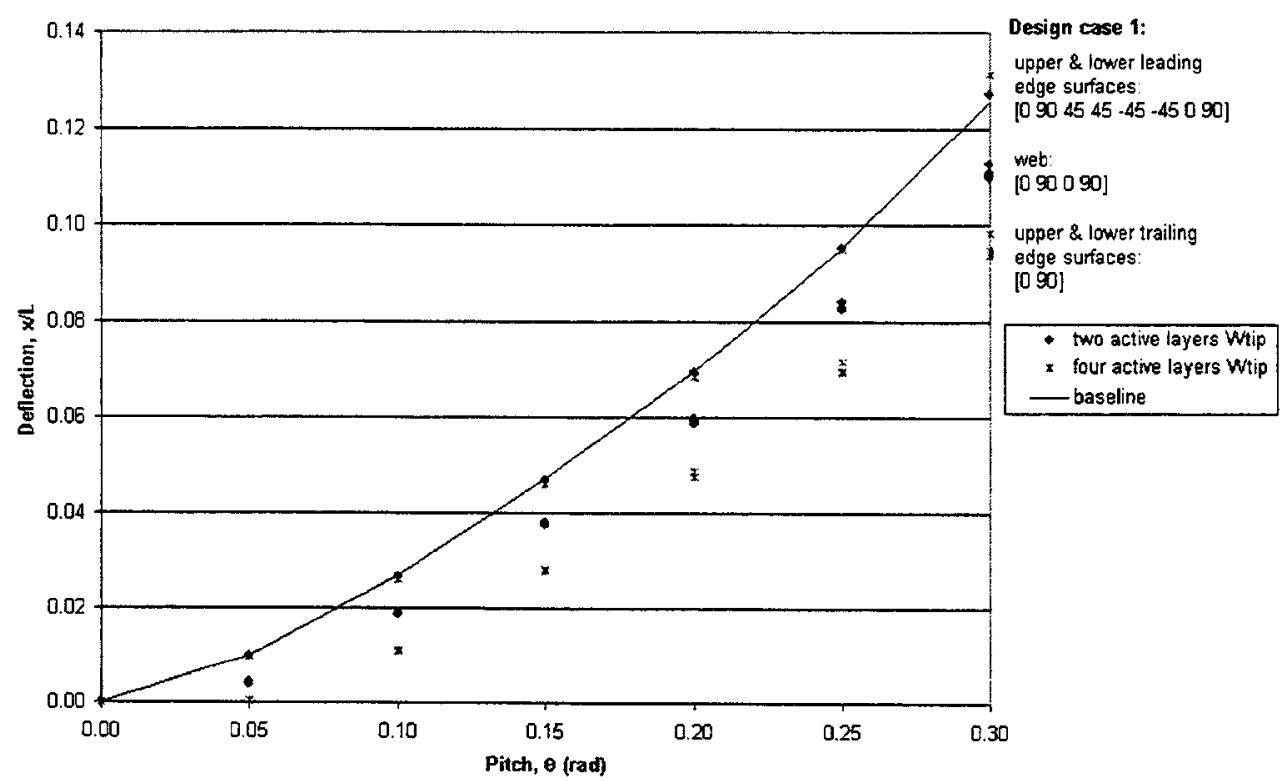

Figure 1.2: Equilibrium tip deflections in the flap bending 


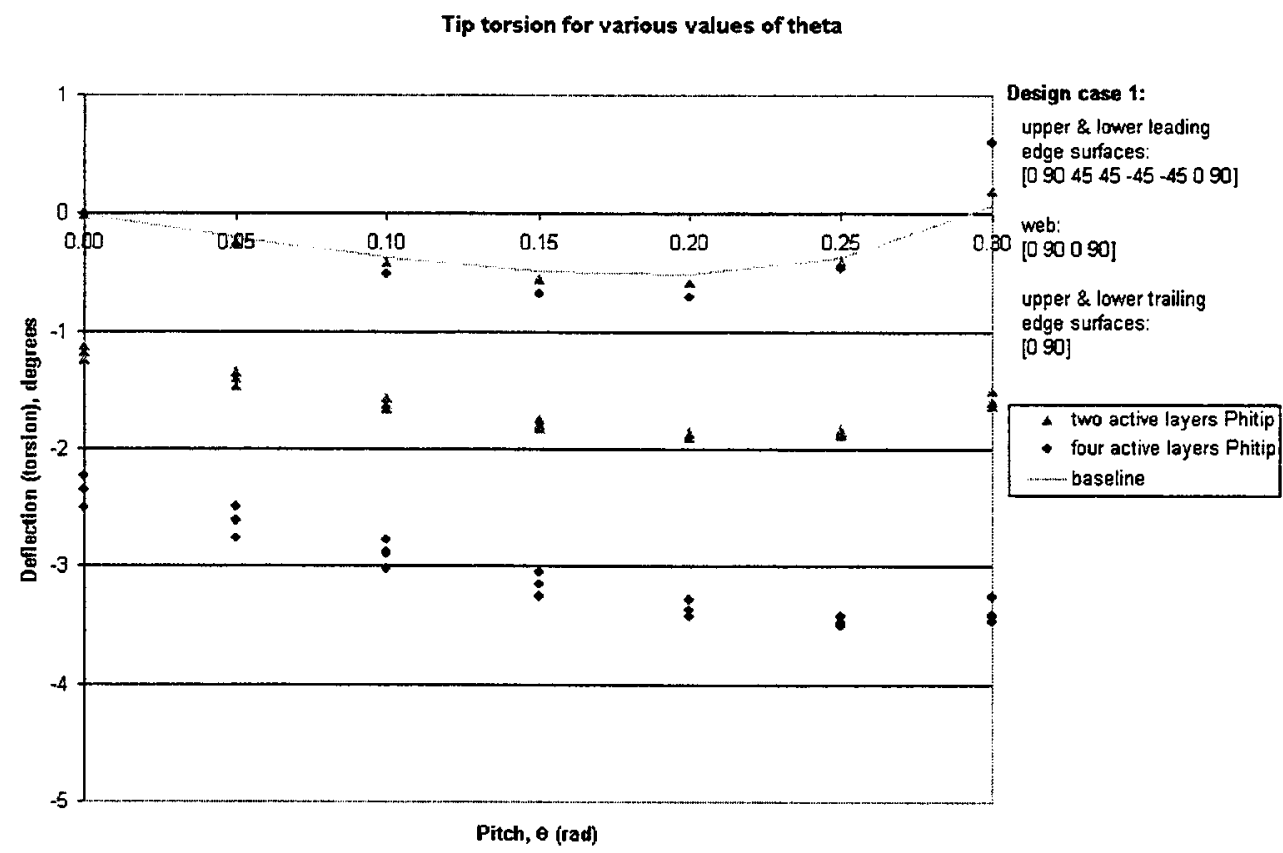

Figure I.3: Equilibrium tip torsion

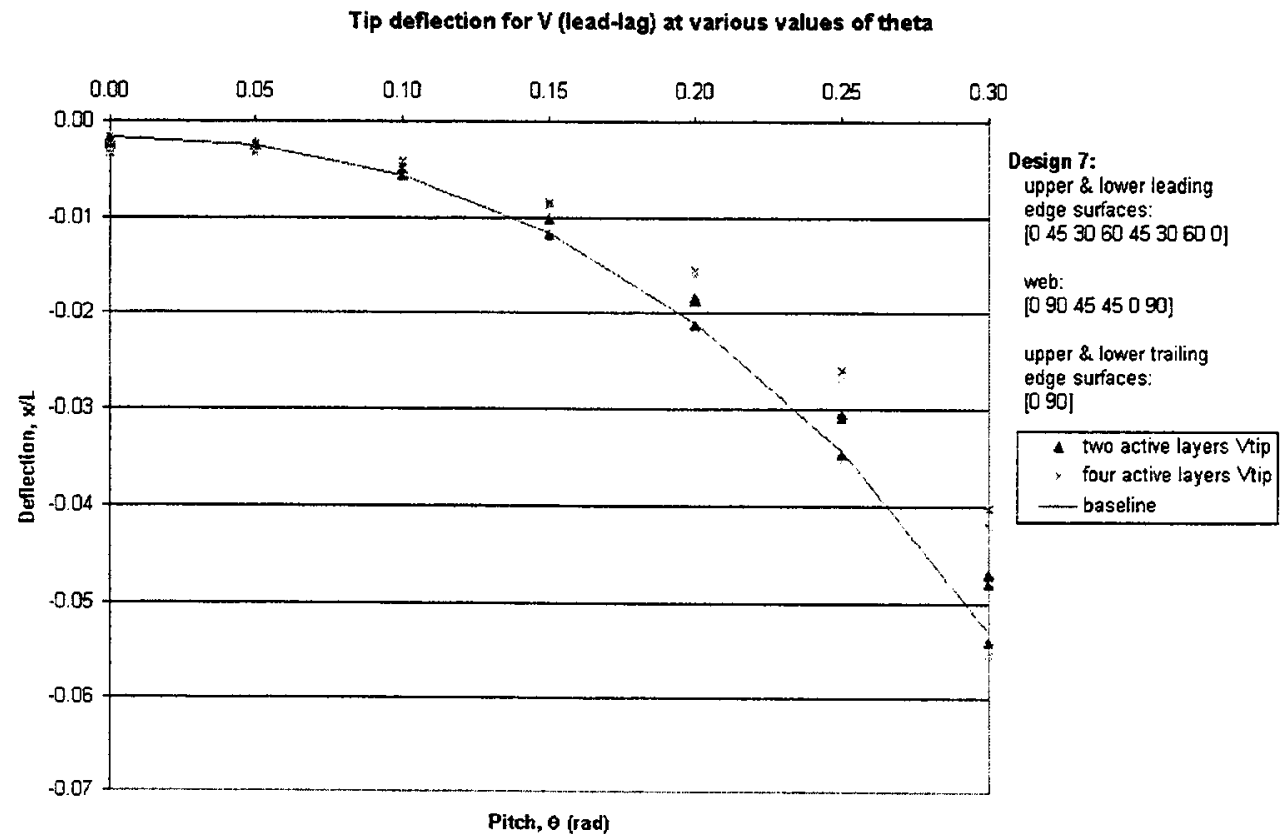

Figure I.4: Equilibrium tip deflections in lead-lag bending 


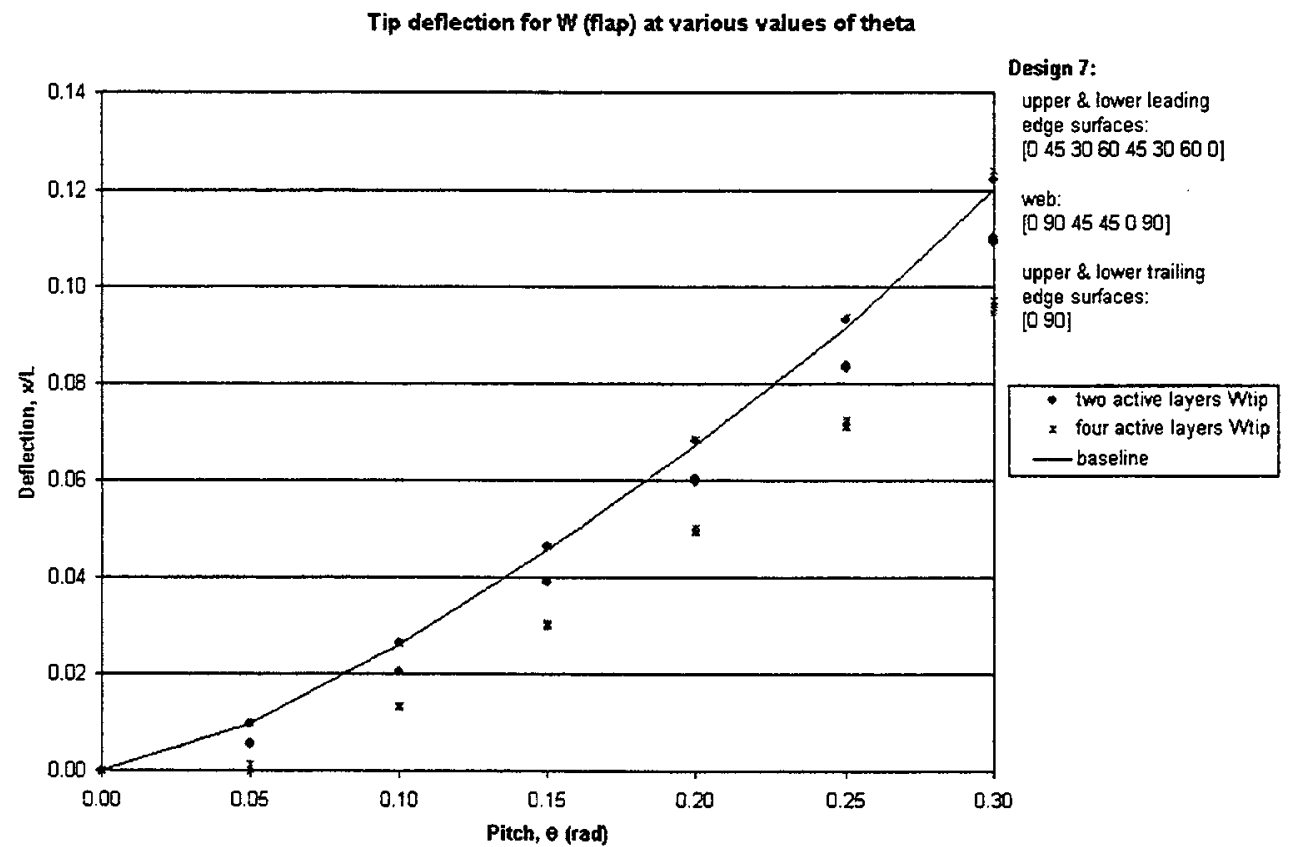

Figure I.5: Equilibrium tip deflections in the flap bending

Tip torsion for various values of theta

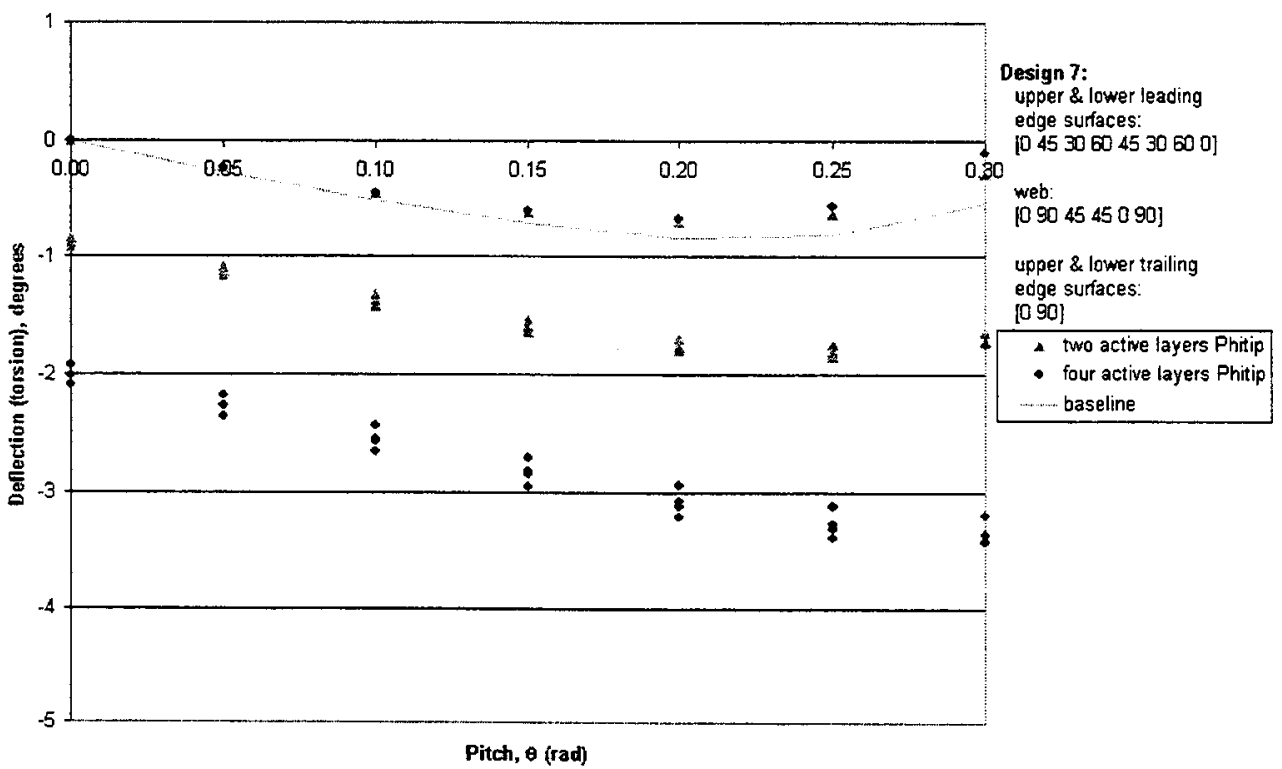

Figure I.6: Equilibrium tip torsion 


\section{Appendix J}

\section{Lead-Lag and Flap Deflections}

The following graphs display the lead-lag and flap deflections experienced by Design 8 .

They are shown as a function of blade span, percent of span actuated, and pitch angle. 


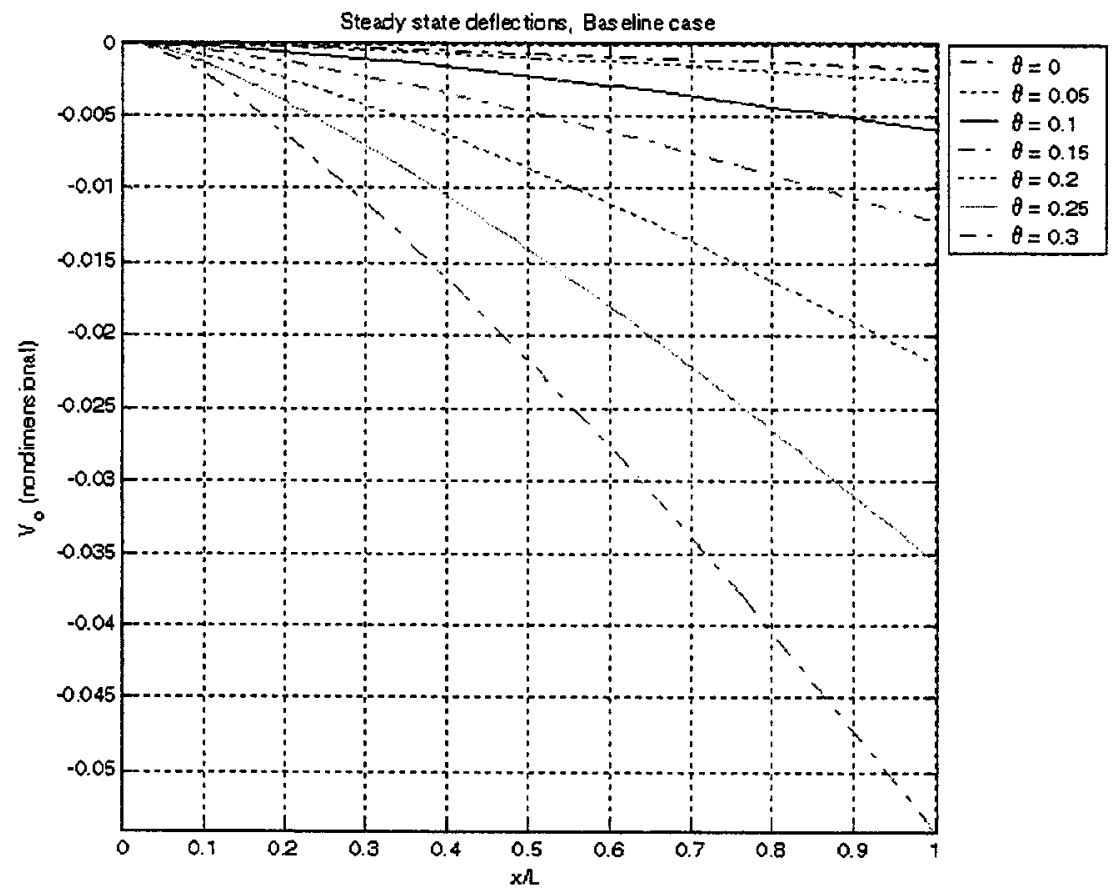

Figure J.1: Lead-lag deflection for the baseline case of Design 8

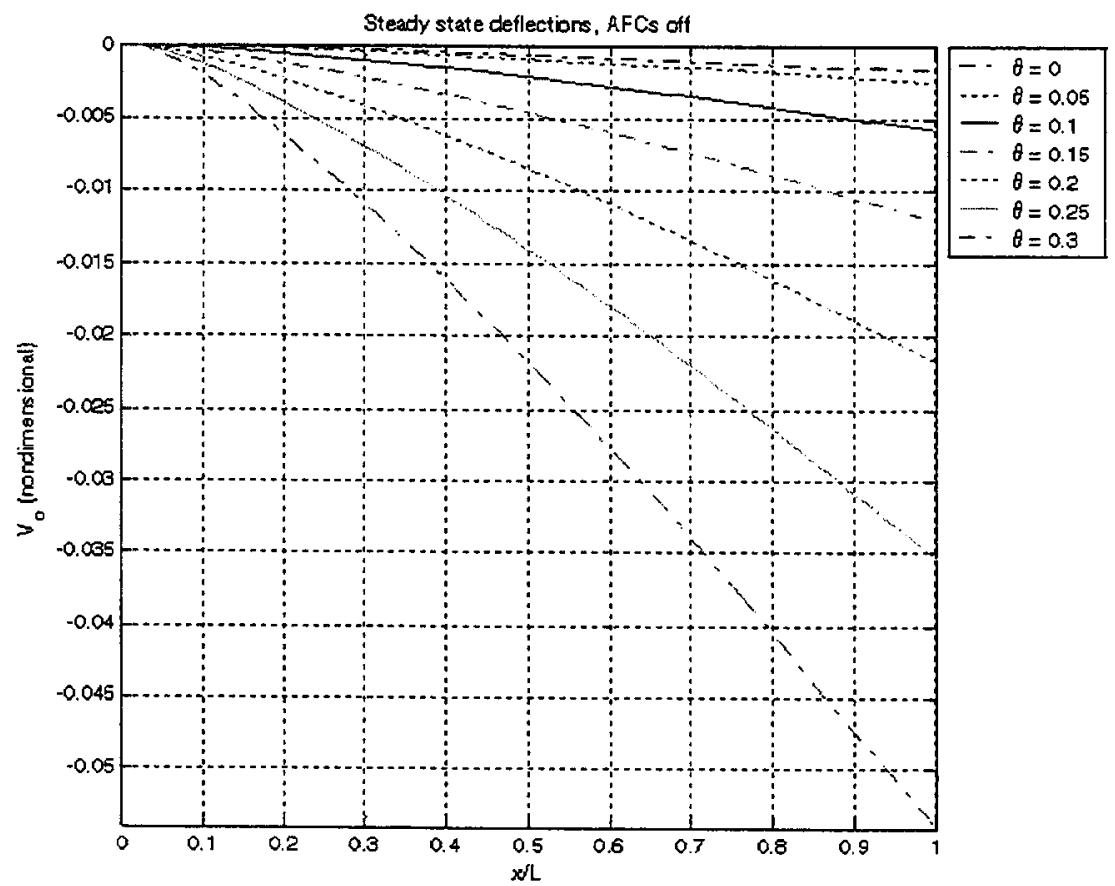

Figure J.2: Lead-lag deflection for zero applied load in Design 8 


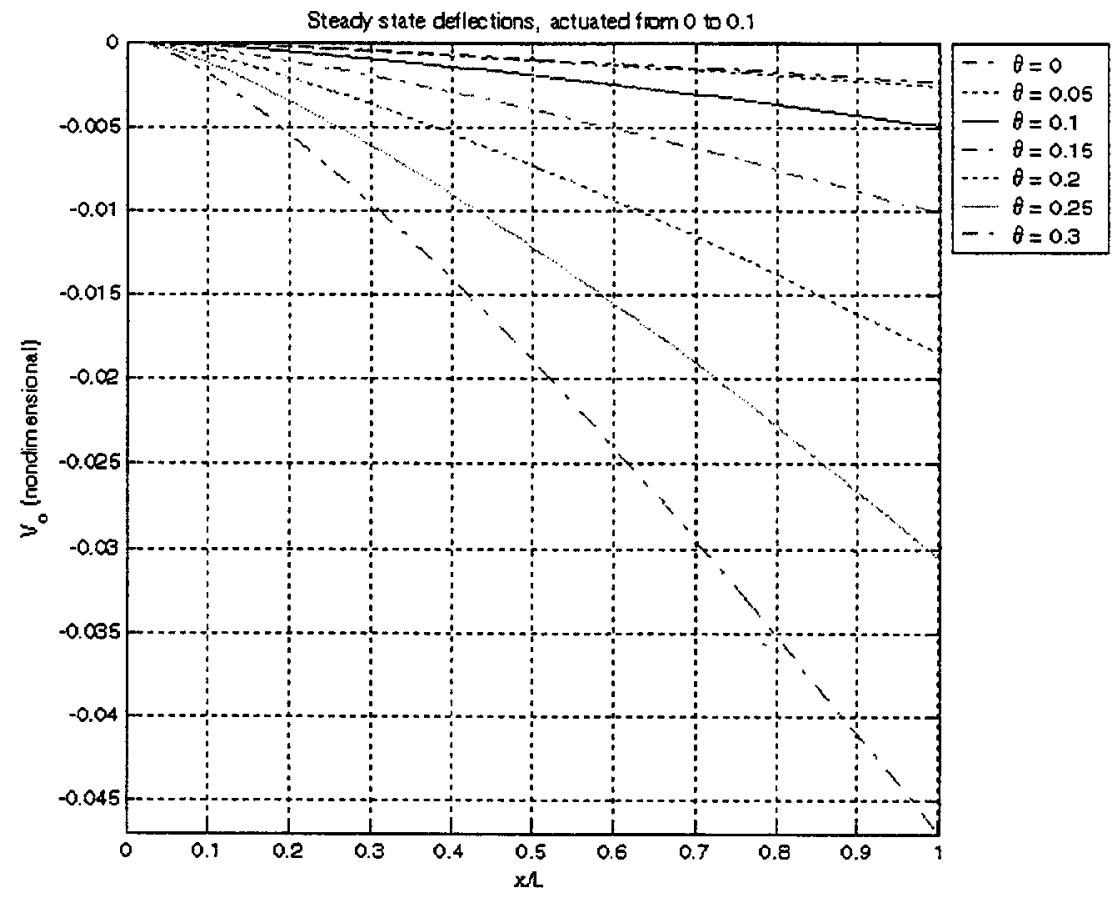

Figure J.3: Lead-lag deflection for $10 \%$ span-wise actuation in Design 8

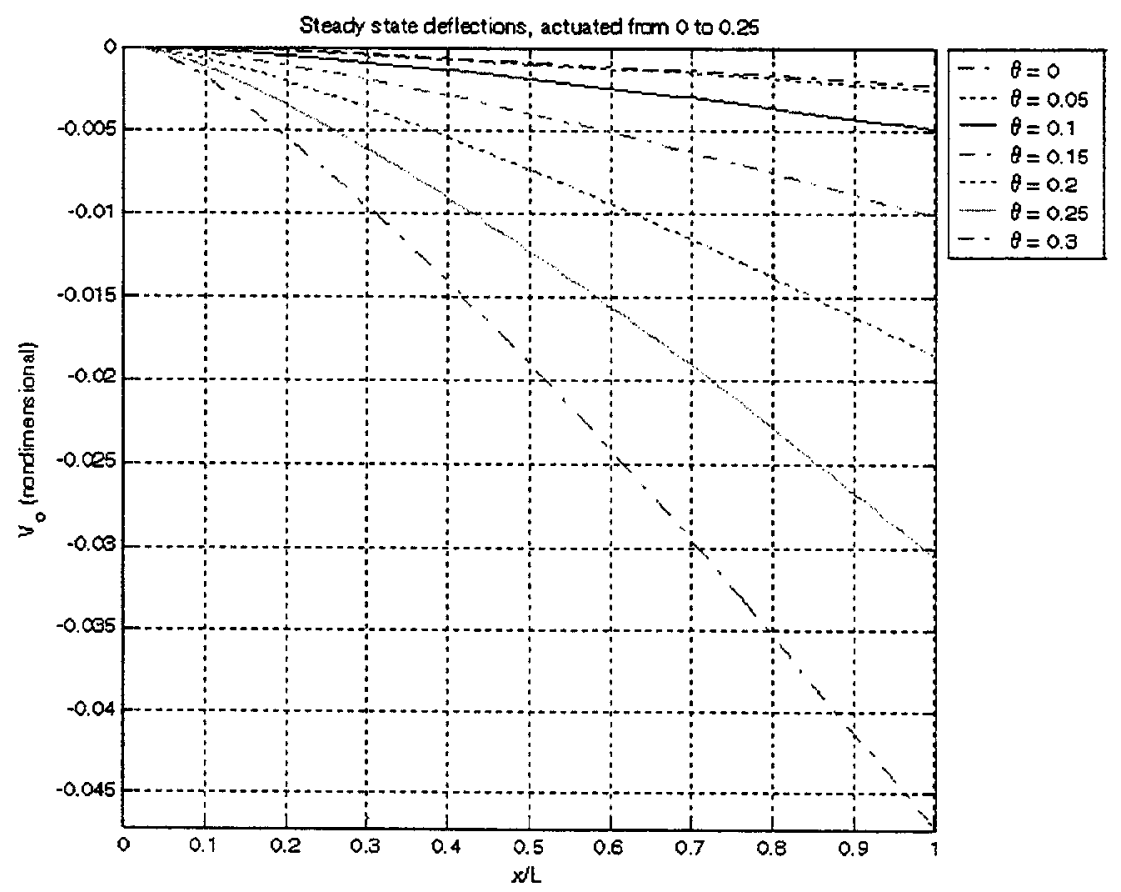

Figure J.4: Lead-lag deflection for $25 \%$ span-wise actuation in Design 8 


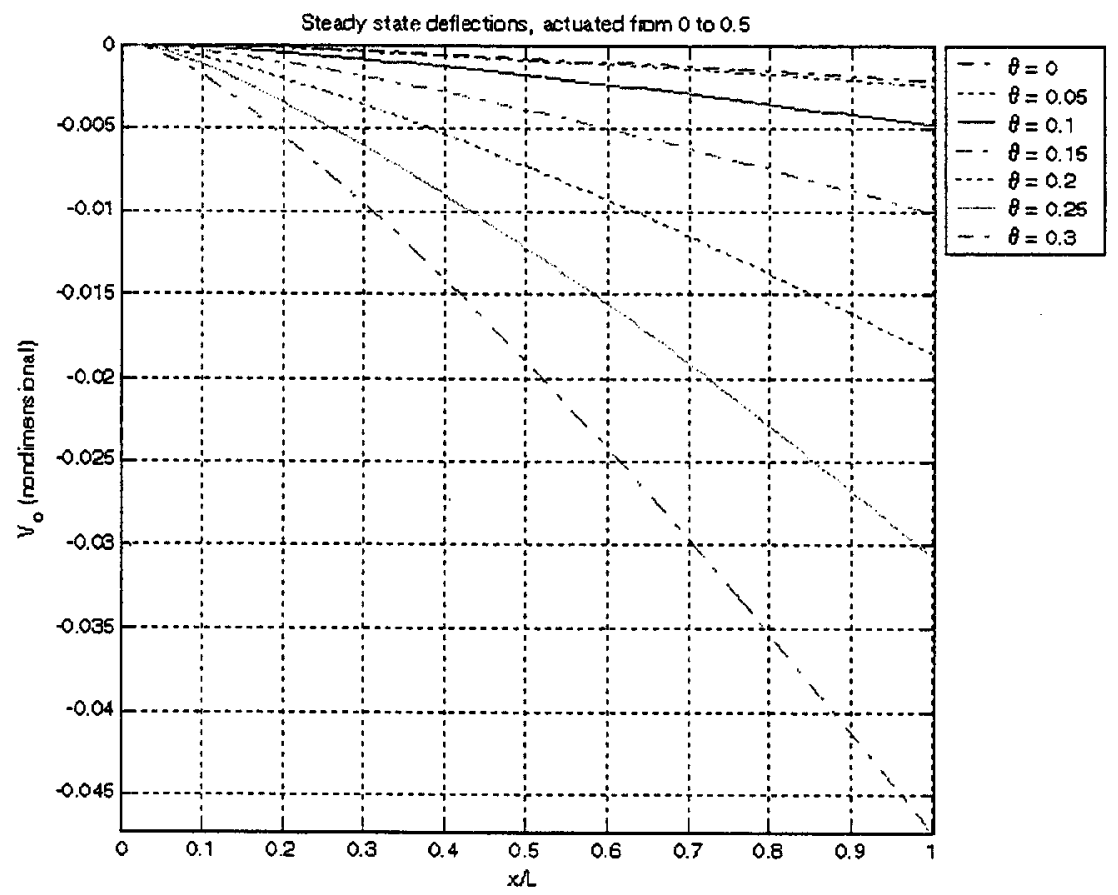

Figure J.5: Lead-lag deflection for 50\% span-wise actuation in Design 8

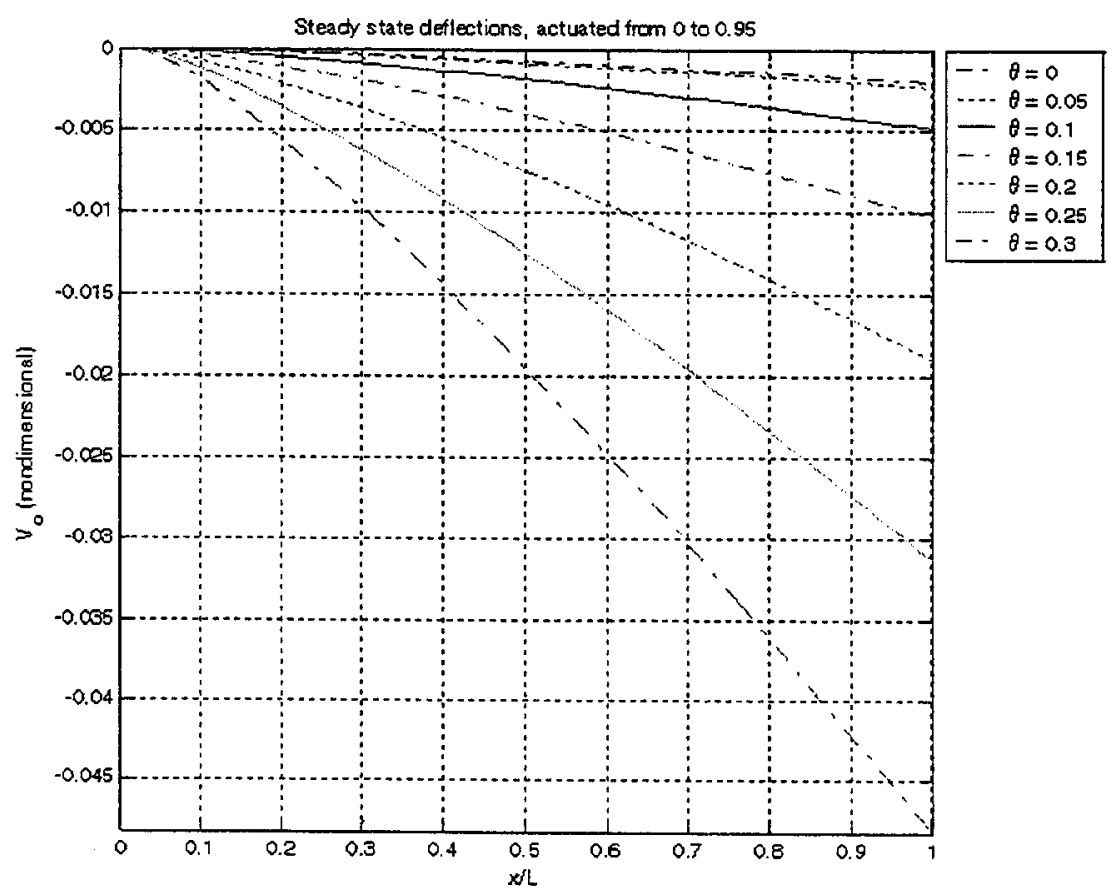

Figure J.6: Lead-lag deflection for $95 \%$ span-wise actuation in Design 8 


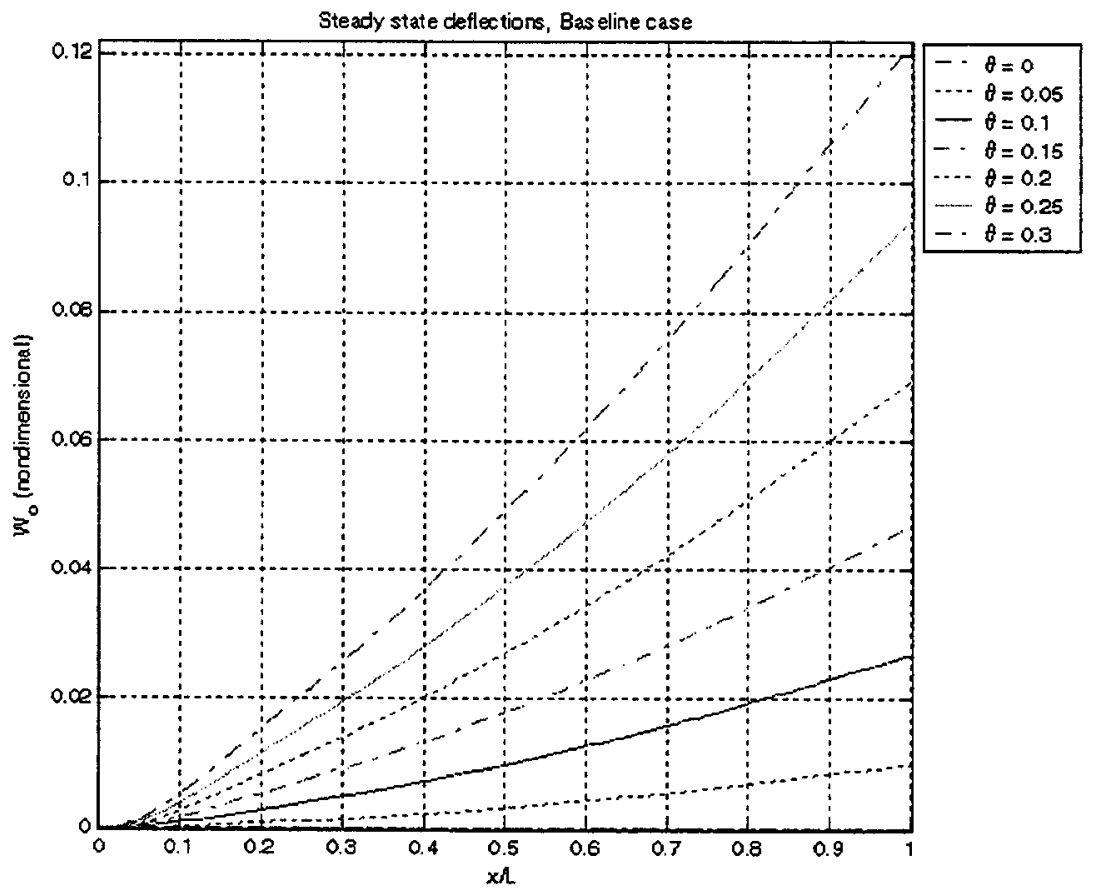

Figure J.7: Flap deflection for the baseline case of Design 8

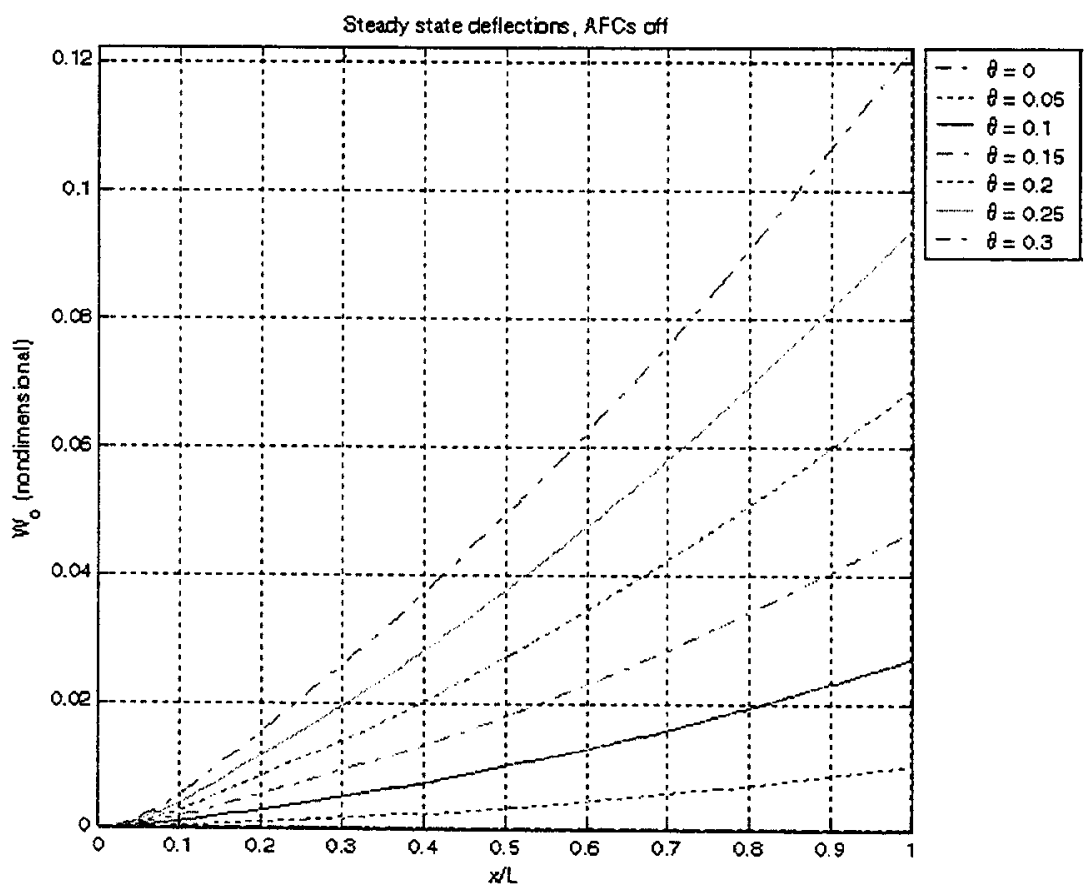

Figure J.8: Flap deflection for zero applied load in Design 8 


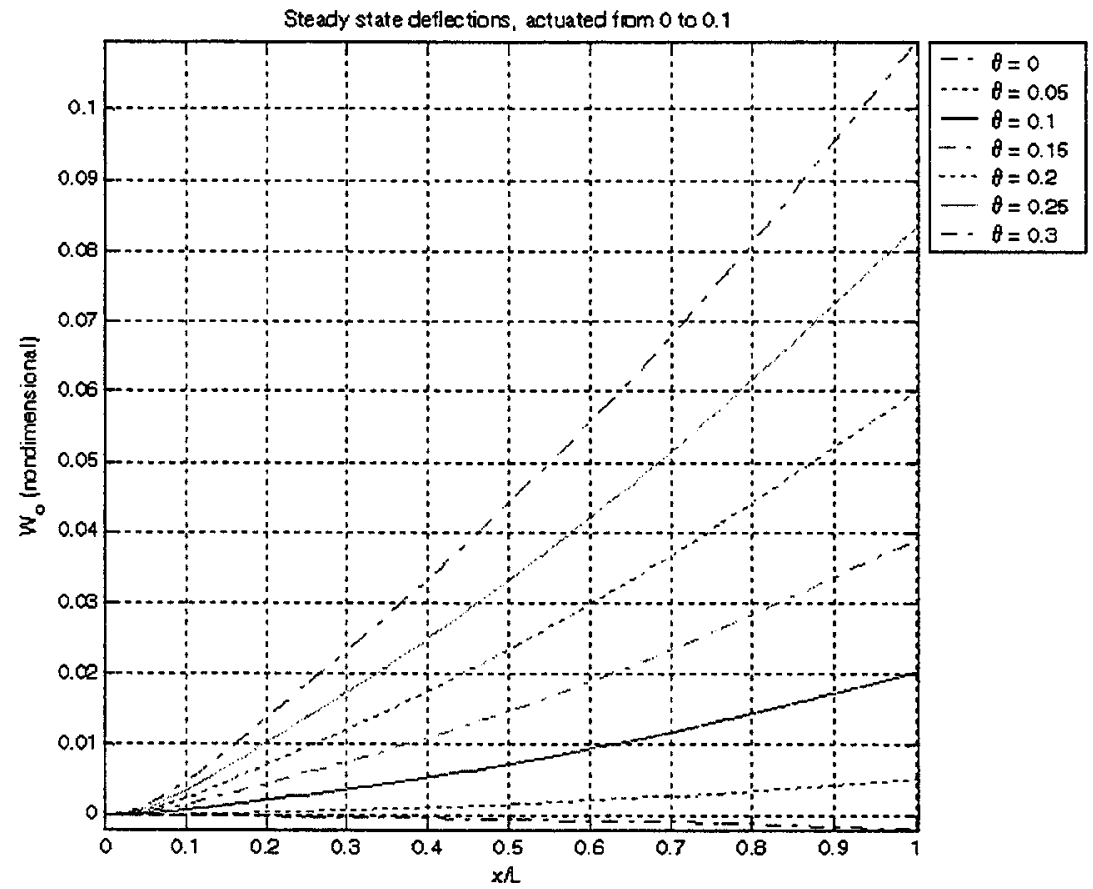

Figure J.9: Flap deflection for 10\% span-wise actuation in Design 8

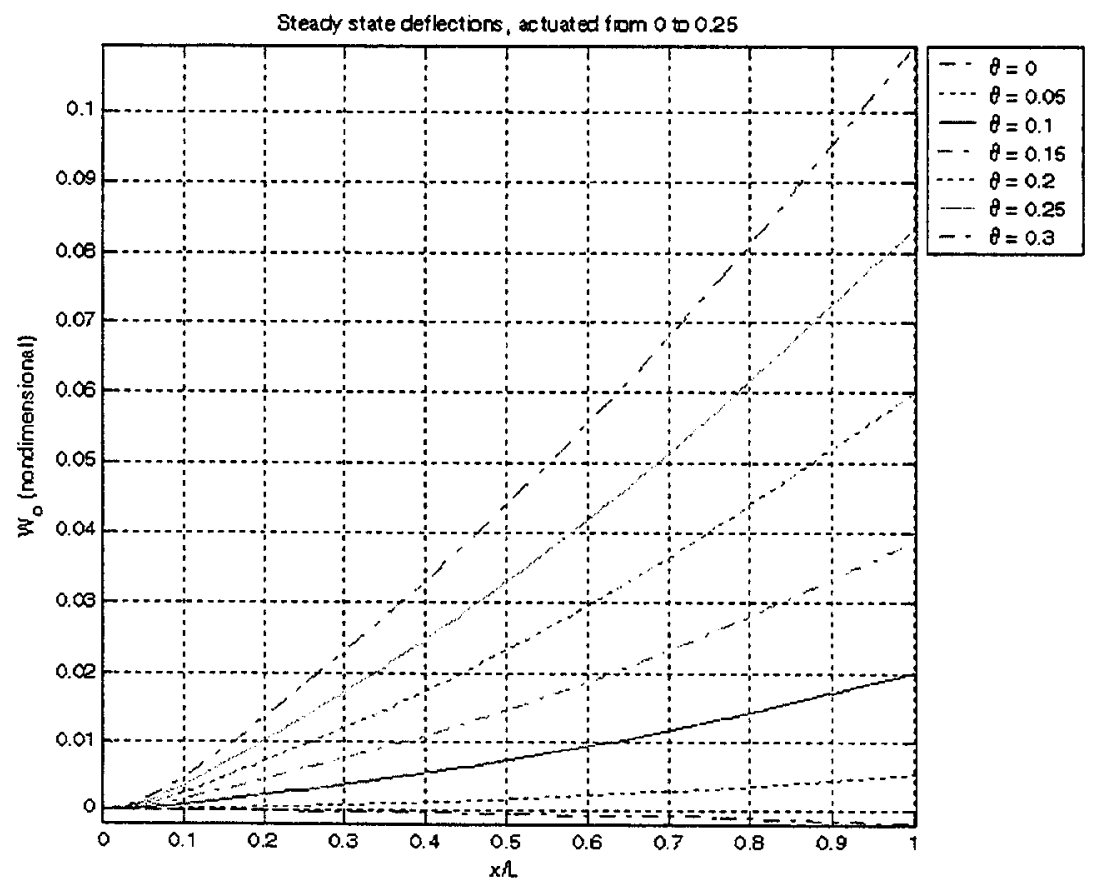

Figure J.10: Flap deflection for $25 \%$ span-wise actuation in Design 8 


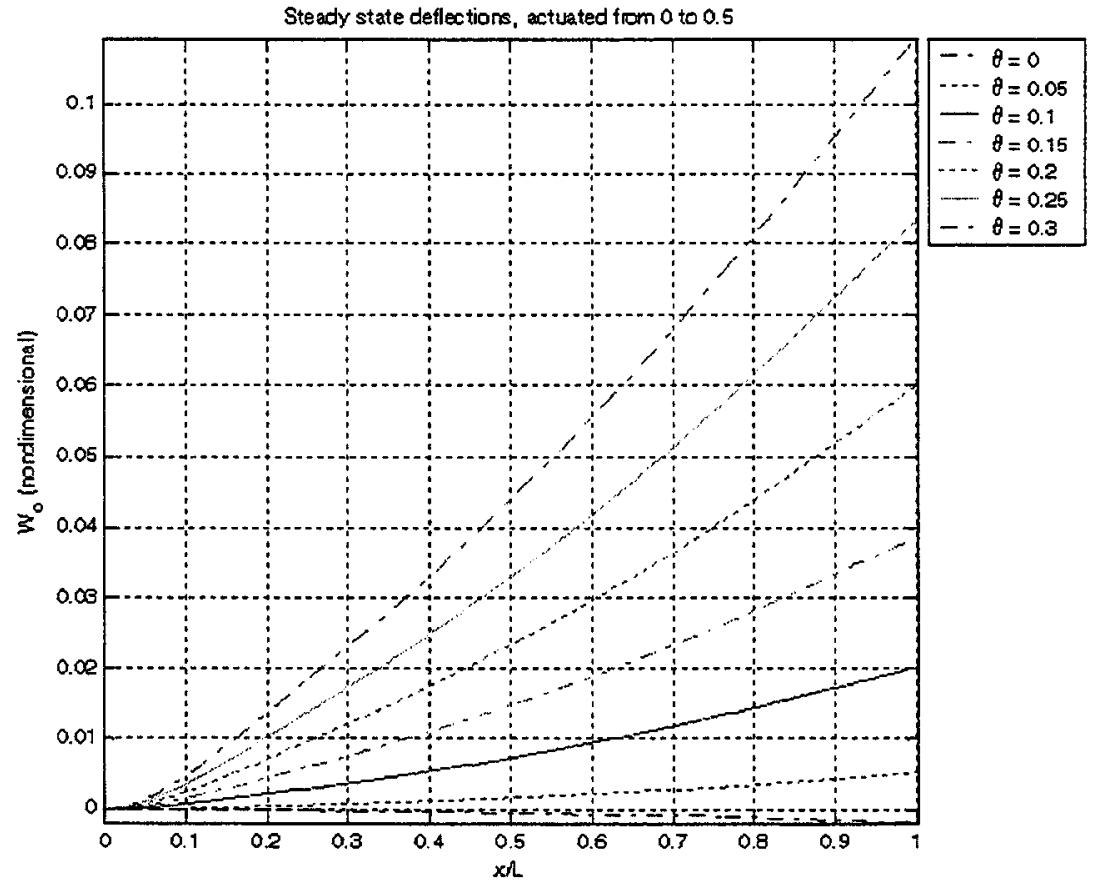

Figure J.11: Flap deflection for $50 \%$ span-wise actuation in Design 8

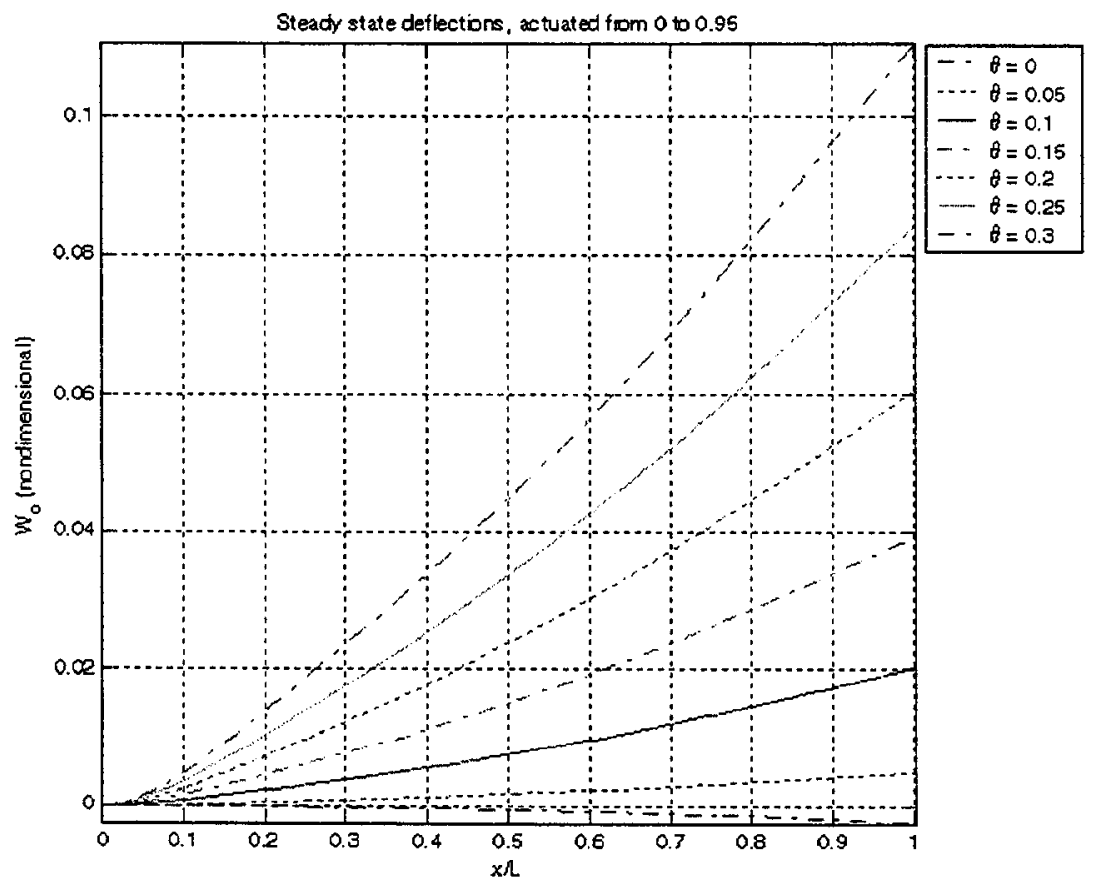

Figure J.12: Flap deflection for $95 \%$ span-wise actuation in Design 8 


\section{Appendix K}

\section{Box Beam Code}

This section outlines the computer codes that were developed in MATLAB version 6.5 for the purposes of modelling the two-celled box beam blade described in Section 4.4.2. Figure K.1 illustrates a map of all pertinent .m files and output files for this cross-sectional analysis. The complete code is provided in the $\mathrm{CD}$ accompanying this thesis.

\section{design.m}

- Is the initialising and main program for the box beam model.

- Runs through a series of sizes of box beams in order to converge on a solution that will fit into a NACA 0012 airfoil section and conform to the design requirements for blade stiffness.

- Requires the input file 'KvalsDUM.in', and outputs 'lastinfo.xls'.

- Calls up programs: 'sidethicks.m', 'boxdummy.m', and 'NACA0012r.m'.

sidethicks.m

- Calculates the total thickness of each of the sides of the box beam. 


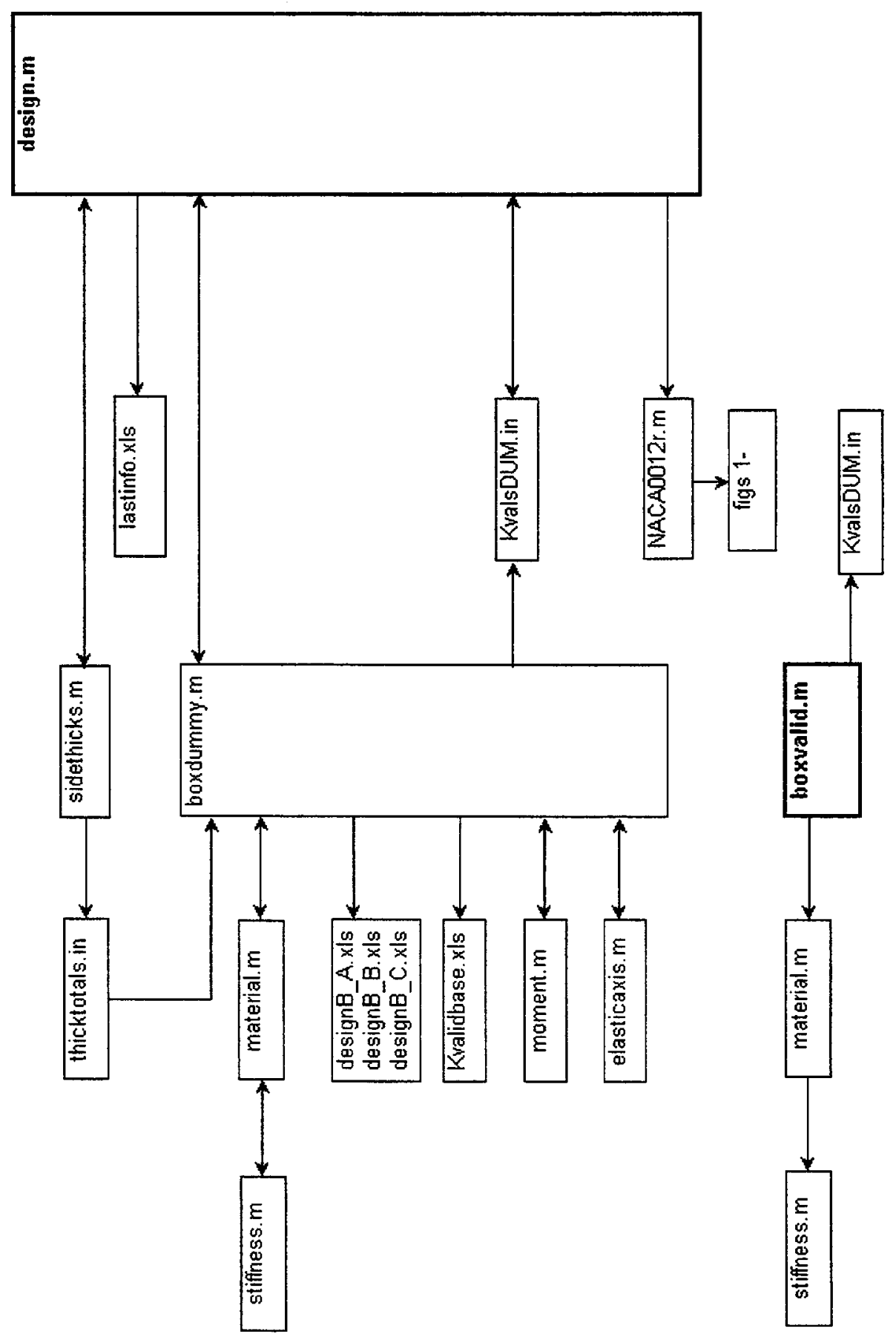

Figure K.1: Program map for box beam blade 
- Provides output file 'thicktotals.in'.

\section{boxdummy.m}

- Provides the material properties, angles, no. layers, thicknesses, for each side and calculates the stiffness constants, $K_{i j}$.

- Requires input file 'thicktotals.in'.

- Calls up 'material.m', 'moment.m', and 'elasticaxis.m'.

- Outputs design results in 'Kvalidbase.xls', 'designB_A.xls', 'designB_B.xls', 'designB_C.xls', and 'KvalsDUM.in'.

material.m

- Calculates the $A, B$, and $C$ stiffness values.

- Calls up 'stiffness.m'.

stiffness.m

- Calculates the Dijkl matrix by using classical laminate theory.

moment.m

- Calculates the moments of inertia, $\mathrm{I}_{y}$ and $\mathrm{I}_{z}$, and the polar moment of inertia, $\mathrm{J}$.

elasticaxis.m

- Determines the location of the elastic axis of the two-celled box beam. 


\section{NACA0012r.m}

- Plots the box beam designs that fall under the design limits within the NACA 0012 airfoil section.

boxvalid.m

- Is the initialising and main program that is used solely for validating the example box beam given in Cesnik and Shin [36].

- Calls up 'material.m', and therefore 'stiffness.m', and outputs 'Kvalid.xls'. 


\section{Appendix L}

\section{NACA 23012 Airfoil Code}

This section outlines the computer codes that were developed in MATLAB version 6.5 for the purposes of modelling the two-celled NACA 23012 airfoil blade described in Section 4.4.3. Figure L.1 illustrates the program map for the .m files that calculate the blade stiffness parameters. Figure L.2 illustrates the series of programs used in analysing the blade for stability purposes. These two sets of programs are linked by the program 'baseline.in', which is outputted from the first set of programs and inputted into the second set of programs. The complete code is provided in the CD accompanying this thesis. 


\section{L.1 Blade Design Code}

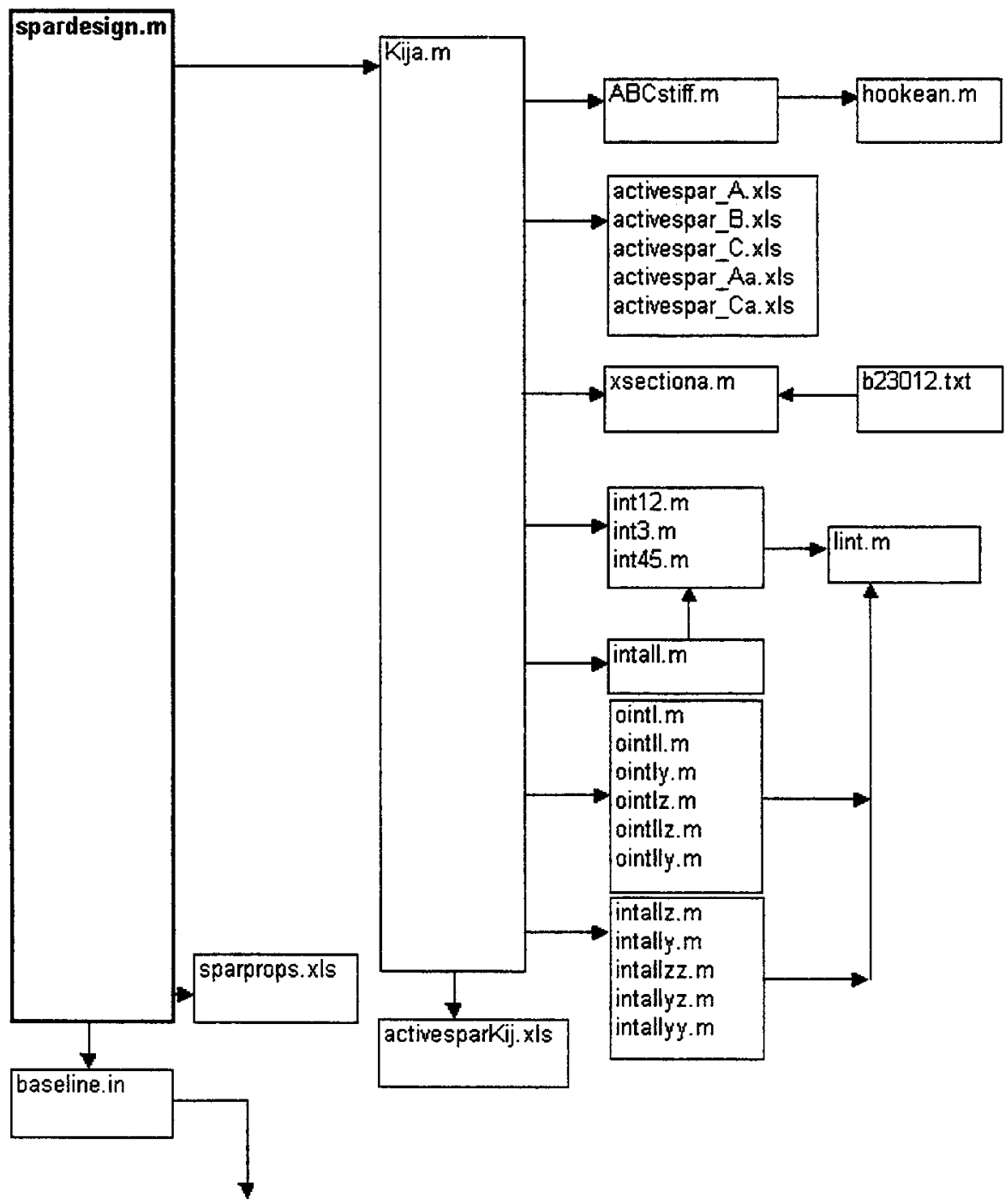

Figure L.1: Program map for NACA 23012 blade 
spardesign.m

- This is the initialising and main program for the design of the NACA 23012 blade design.

- Much like 'design.m' for the box beam design, this program also calculates the crosssectional parameters.

- Calls up 'Kija.m'.

- Outputs the files 'sparprops.xls' and 'baseline.in', where the latter file is also used and required in the stability programs.

\section{Kija.m}

- Provides the stiffness characteristics for this blade section.

- Calls up programs 'ABCstiff.m', 'xsectiona.m', 'int*.m', 'oint*.m'.

- Outputs the files 'activespar*.xls'.

\section{ABCstiff.m}

- Identical to 'material.m', except that it also calculates the active stiffness properties $\mathrm{A}^{(a)}$ and $\mathrm{C}^{(a)}$.

- Calls up 'hookean.m'

\section{hookean.m}

- Identical to 'stiffness.m'. 


\section{xsectiona.m}

- Divides the spar section into segments in order to calculate the section's centroid and moment of inertia.

- Requires the look-up file 'b23012.txt'.

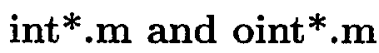

- Includes the .m files: 'int12.m', 'int3.m', 'int45.m', 'intall.m', 'intallz.m', 'intally.m', 'intallzz.m', 'intallyz.m', 'intallyy.m'.

- Also includes the .m files: 'ointI.m', 'ointII.m', 'ointIy.m', 'ointIz.m', 'ointIIy.m', 'ointIIz.m'

- Integration over branches of cross-section for integrals defined in Appendix B.

- Calls up the file 'lint.m'.

lint.m

- Numerical integration for the above 'int*.m' and 'oint*.m' files, using the Trapezoidal rule. 


\section{L.2 Stability Code}

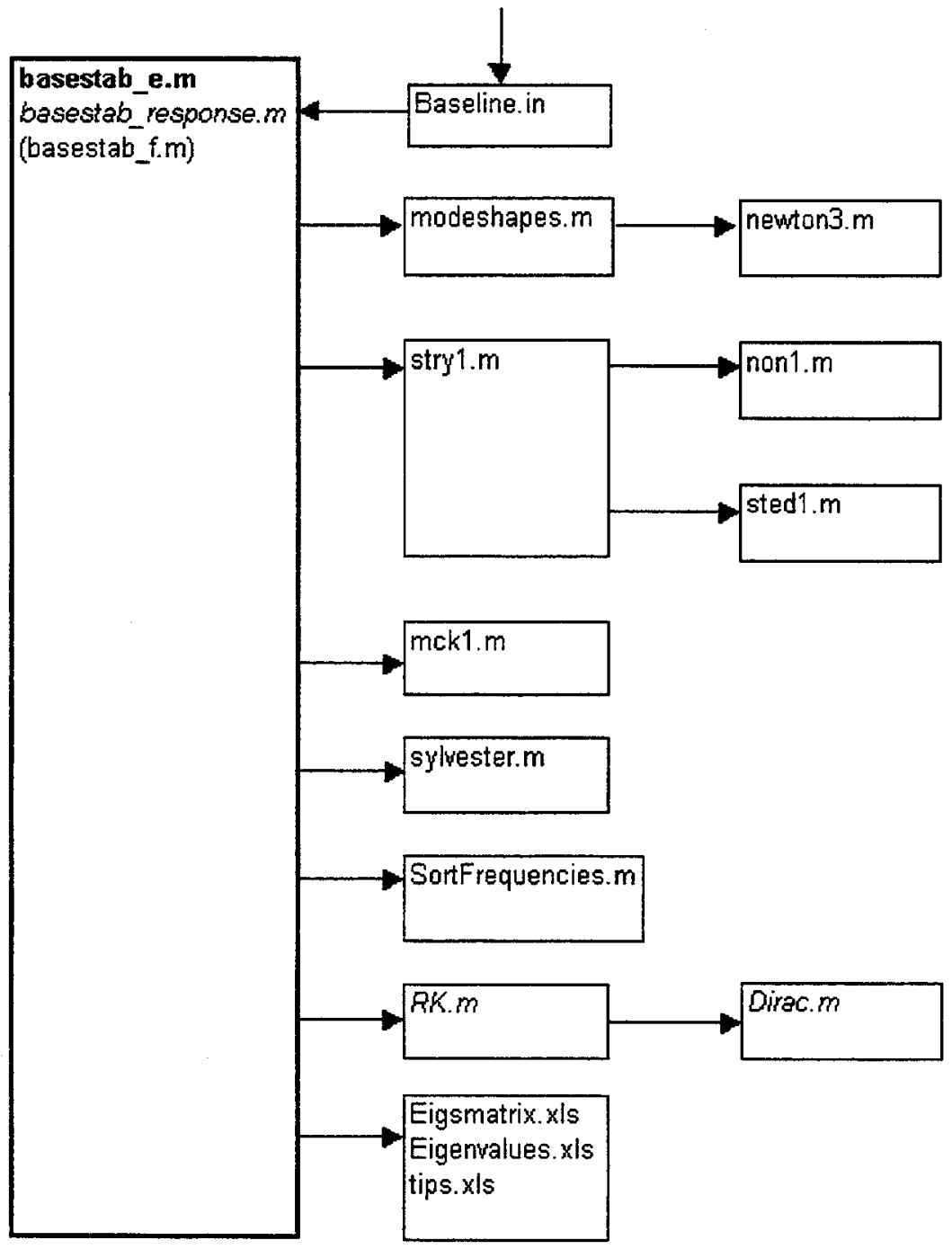

Figure L.2: Program map for stability analysis of NACA 23012 blade 


\section{basestab_e.m}

- Is the initialising and main program for the stability analysis of the blade.

- Determines the eigenvalues/natural frequencies of a blade design by determining the mass, damping, and stiffness matrices.

- Requires the input file 'baseline.in' from the output of the design program 'spardesign.m'.

- Calls up 'modeshapes.m', 'stry1.m', 'mck1.m', 'sylvester.m', 'SortFrequencies.m'.

- Outputs the files 'Eigsmatrix.xls', 'Eigenvalues.xls', 'tips.xls'.

\section{modeshapes.m}

- Calculates the inactive and active mode shapes defined in Appendix F.

- Calls up the file 'newton3.m'

\section{newton3.m}

- Numerical integration program that utilises Newton's method.

stry1.m

- Solver for the trim deflections $\mathrm{V}_{0 j}, \mathrm{~W}_{0 j}$, and $\Phi_{0 j}$.

- Calls up the programs 'non1.m' and 'sted1.m'.

sted1.m

- Defines the steady-state deflections of the non-actuated and actuated rotor blades defined in Chapter 3, Eqs. (3.41) to (3.43). 
non1.m

- Similar to 'sted1.m' except that it only carries out the function for a non-actuated blade.

mck1.m

- Creates the mass, damping, and stiffness matrices for the active and inactive blades.

sylvester.m

- Checks to see if a matrix is positive definite or not.

\section{SortFrequencies.m}

- Sorts the eigenvalues in ascending order.

\section{basestab_response.m}

- Has the same purpose as 'basestab_e.m', except that it also analyzes the response of an impulsive actuation, defined in Section 5.4.2.

- Calls up the same programs as 'basestab_e.m' as well as the programs 'RK.m', and therefore 'Dirac.m'.

- Outputs same files.

RK.m

- Runge-Kutta solver for impulsive actuation.

- Calls up 'Dirac.m'. 


\section{Dirac.m}

- Defines the Dirac-delta function.

\section{basestab_f.m}

- Performs the same functions as 'basestab_e.m' except that it applies to the design cases for $\mathrm{AC}$ currents, and requires the application of the Floquet method to solve for the eigenvalues.

- Calls up also the program 'floquet.m'.

\section{floquet.m}

- Numerical solving function. 